CARLOS HENRIQUE ANDRADE COSTA

\title{
CRIPTOGRAFIA QUÂNTICA EM REDES DE INFORMAÇÃO CRÍTICA - APLICAÇÃO A TELECOMUNICAÇÕES AERONÁUTICAS
}

Dissertação apresentada à Escola Politécnica da Universidade de São Paulo para obtenção do Título de Mestre em Engenharia

São Paulo, SP 


\section{CARLOS HENRIQUE ANDRADE COSTA}

BACHAREL EM FÍSICA COMPUTACIONAL PELA UNIVERSIDADE DE BRASÍLIA, 2006

\section{CRIPTOGRAFIA QUÂNTICA EM REDES DE INFORMAÇÃO CRÍTICA - APLICAÇÃO A TELECOMUNICAÇÕES AERONÁUTICAS}

Dissertação apresentada à Escola Politécnica da Universidade de São Paulo para obtenção do Título de Mestre em Engenharia

Área de Concentração: Sistemas Digitais

Orientador: Prof. Dr. João Batista Camargo Jr.

São Paulo, SP

2008 
Este exemplar foi revisado e alterado em relação à versão original, sob responsabilidade única do autor e com a anuência de seu orientador.

São Paulo, 04 de julho de 2008.

Assinatura do autor:

Assinatura do orientador:

\section{FICHA CATALOGRÁFICA}

\section{Costa, Carlos Henrique Andrade}

Criptografia quântica em redes de informação crítica - aplicação a telecomunicações aeronáuticas / C.H.A. Costa. - ed.rev. São Paulo, 2008.

$191 \mathrm{p}$.

Dissertação (Mestrado) - Escola Politécnica da Universidade de São Paulo. Departamento de Engenharia de Computação e Sistemas Digitais.

1.Criptologia 2.Rede de telecomunicações 3.Informação (Segurança)

4.Computação quântica I.Universidade de São Paulo. Escola Politécnica. Departamento de Engenharia de Computação e Sistemas Digitais II.t. 
Aos meus pais, esposa e mestres. 


\section{Agradecimentos}

Ao meu orientador Prof. João Batista Camargo Jr., sou grato pela orientação, pela disposição e atenção no acompanhamento das diversas etapas desta pesquisa.

Aos professores do Departamento de Engenharia de Computação e Sistemas Digitais e do Departamento de Engenharia de Sistemas Eletrônicos que contribuíram em meu aprofundamento no tema abordado, em especial ao Prof. Paulo Barreto pelos valiosos conselhos em diversos tópicos do trabalho.

Aos demais colegas de pós-graduação, pelas críticas e sugestões.

À minha esposa pelo apoio e compreensão durante o desenvolvimento deste trabalho e à minha família em geral, responsável pela essência de minha formação e sempre uma fonte de apoio incondicional.

Ao CNPq, pelo apoio financeiro, e ao Grupo de Análise de Segurança, pelo compartilhamento de um ambiente propício à pesquisa acadêmica e da estrutura física necessária para a execução do trabalho. 
A Ciência enseja a mais ousada metafísica de nosso tempo. É um constructo puramente humano, alimentada pela fé de que se sonharmos, buscarmos a descoberta, explicarmos e sonharmos novamente, desbravando repetidamente territórios desconhecidos, o mundo se tornará algo mais compreensível e nós capturaremos a verdadeira estranheza do Universo. E toda essa estranheza se mostrará interconectada e fará sentido 


\section{Resumo}

Ocorre atualmente um movimento de aumento da importância que a manutenção da segurança da informação vem adquirindo em redes de informação de crítica. Ao longo das últimas décadas a utilização de ferramentas criptográficas, especialmente aquelas baseadas em problemas de díficil solução computacional, foram suficientes para garantir a segurança dos sistemas de comunicação. Contudo, o desenvolvimento da nova técnica de processamento de informação conhecida como computação quântica e os resultados téoricos e experimentais apresentados por esta mostram que é possível inviabilizar alguns dos sistemas de criptografia atuais amplamente utilizados. A existência de tal vulnerabilidade representa um fator crítico em redes em que falhas de segurança da informação podem estar associadas a riscos de segurança física. Uma alternativa para os métodos criptográficos atuais consiste na utilização de sistemas quânticos na obtenção de um método criptográfico, o que se conhece como criptografia quântica. Este novo paradigma tem seu fundamento resistente mesmo na presença de capacidade tecnológica ilimitada, incluindo o cenário com disponibilidade de computação quântica. Este trabalho tem como objetivo levantar os impactos que o desenvolvimento da computação quântica têm sobre a segurança dos atuais sistemas criptográficos, apresentar e desenvolver alternativas de protocolos de criptografia quântica disponíveis, e realizar um estudo de caso por meio da avaliação da utilização de criptografia quântica no contexto da Aeronautical Telecommunication Network (ATN). Isto é feito por meio do desenvolvimento de um ambiente de simulacão que permite avaliar o comportamento de um protocolo de criptografia quântica em um cenário em um ambiente com requisitos de missão crítica, como é o caso da ATN.

Palavras-chave: criptografia quântica, redes de informação crítica, telecomunicações aeronáuticas, computação quântica. 


\section{Abstract}

The importance of security maintenance in critical information networks has been rising in recent times. Over the past decades, the utilization of cryptography tools, mainly those based on computationally intractable problems, was enough to ensure the security of communications systems. The development of the new information processing technique known as quantum computation and the theoretical and experimental results showed by this approach demonstrated that could be possible to cripple the current widely used cryptography techniques. This vulnerability represents a critical issue for networks where a security fault could be associated to a safety fault. An alternative for the current cryptography methods consists in the utilization of quantum systems to obtain a new cryptographic method. The new paradigm presented by this approach has solid principles even in the presence of unlimited computational capacity, including the scenario with availability of quantum computation. The aim of this work is the assessment of impacts that the development of quantum computation has over the current cryptographic methods security, the presentation and development of alternatives based on quantum cryptography protocols, and the development of a case study using the case of Aeronautical Telecommunication Network (ATN). This aim is reached by means of the development of a simulation environment that allows the evaluation of a quantum cryptography protocol behavior in an environment with mission critical requirements, like the ATN case.

Keywords: quantum cryptography, critical information networks, aeronautical telecommunications, quantum computation 


\section{Lista de Figuras}

1 Conceito básico de transmissão segura de informação . . . . . . . . . p p. 30

2 Sistema criptográfico simétrico em redes móveis públicas . . . . . . p. 34

3 Encriptação com um gerador de chaves . . . . . . . . . . p. 36

4 Esquema de implementação do conceito de Diffie-Hellman . . . . . . p. 39

5 Arquitetura de implementação do conceito de assinatura digital . . . p.44

6 Representação de um qubit na esfera de Bloch . . . . . . . . . . p. 52

7 Exemplos de portas clássicas e quânticas . . . . . . . . . . . . . p. 59

8 Porta quântica CNOT . . . . . . . . . . . . . . . p. 59

9 Circuito quântico para criar estados de Bell . . . . . . . . . . . . p. 60

10 Representação diagramática da porta de Toffoli . . . . . . . . . . . p. 61

11 Circuito para implementação do algoritmo de Deutsch . . . . . . . p. 63

12 Circuito quântico para Transformada Quântica de Fourier . . . . . p. 68

13 Circuito quântico para Estimativa de Fase . . . . . . . . . . . p. 70

14 Número de operações $\sigma$ requisitado para quebrar uma chave RSA de tamanho $N$ utilizando diferentes métodos $\ldots \ldots \ldots$. . . . . . . . . .

15 Tempo $s$ em segundos requisitado para quebrar uma chave RSA de tamanho $N$ utilizando diferentes métodos $\ldots \ldots \ldots$. . . . . . . . . .

16 Bases de codificação para o protocolo BB84 . . . . . . . . . . p. 84

17 Passos da execução do protocolo BB84 no caso ideal . . . . . . . . . p. 86

18 Passos da execução do protocolo BB84 na presença de Eva $\ldots$. . . . . p. 87 
19 Estrutura para distribuição de chaves quânticas utilizando satélites . p. 108

20 Estrutura de roteamento da ATN . . . . . . . . . . . p. 117

21 IDCQ baseada em solo . . . . . . . . . . . . . . . . . p. 124

22 IDCQ baseada em solo utilizando encaminhador DCQ . . . . . . . . p. 124

23 IDCQ baseada em aeronave . . . . . . . . . . . . p. 125

24 IDCQ baseada em aeronave utilizando encaminhador DCQ . . . . . . p. 125

25 IDCQ baseada em satélites . . . . . . . . . . . . . p. 126

26 IDCQ baseada em satélites utilizando encaminhadores DCQ . . . . . p. 127

27 IDCQ aplicação solo/solo . . . . . . . . . . . . . . . . p. 128

28 IDCQ baseada em encaminhadores de dados DCQ em solo . . . . . . p. 129

29 IDCQ baseada em satélites agindo como encaminhadores de dados

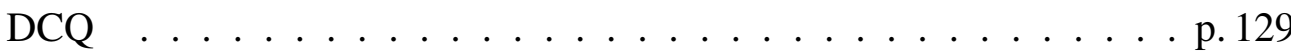

30 IDCQ baseada em satélites agindo como encaminhadores de dados DCQ com múltiplas rotas $\ldots \ldots \ldots \ldots$. . . . . . . . . 130

31 Tamanho da sequência de bases corretas BC e chave final SK obtida em 100 realizações do protocolo BB84 com valores médios $\langle B C>$ $\mathrm{e}<S K>\ldots \ldots \ldots \ldots \ldots \ldots \ldots \ldots \ldots \ldots$

32 Erro $e$ e erro médio $<e>$ observado em 100 realizações do protocolo BB84 na presença de interferência completa . . . . . . . . . . p. 147

33 Erro aleatório físico gerado $e_{f}$ e erro medido $e_{m}$ em 100 realizações do protocolo BB84 na ausência de interferência com valores médios

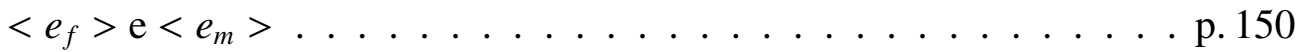

34 Erro aleatório físico gerado $e_{f}$ e erro medido $e_{m}$ em 100 realizações do protocolo $\mathrm{BB} 84$ na presença de interferência total com valores médios $<e_{f}>\mathrm{e}<e_{m}>\ldots \ldots \ldots \ldots \ldots \ldots \ldots$

35 Execução do algoritmo Cascade para 10 casos distintos . . . . . . . . p. p. 154 
36 Função de distribuição de probabilidade de $x$ ocorrências na distribuição de Poisson para uma média de intensidade de fótons $\mu$. . . p. 156

37 Probabilidade de ao menos uma ocorrência na distribuição de Poisson em função da intensidade $\mu \ldots \ldots \ldots \ldots$. . . . . . . 156

38 Relação entre o tamanho final da chave obtida após o processo de amplificação de privacidade em função do tamanho inicial da sequencia

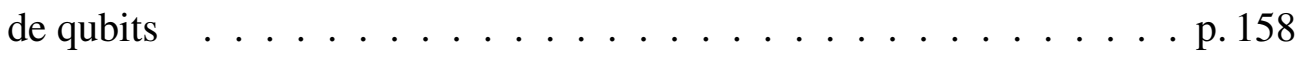

39 Referências técnicas para tecnologias de comunicação utilizadas na ATN a partir de acordo com a especificação ICAO (ICAO, 2002) . . p. 164 


\section{Lista de Tabelas}

1 Tabela-verdade da porta de Toffoli . . . . . . . . . . . . p. 61

2 Tabela-verdade da porta CNOT controlada por $f \ldots \ldots$. . . . . . 63

3 Métodos utilizados para quebrar o RSA e complexidade requerida . . p.74

4 Realizações do protocolo BB84 em um cenário ideal . . . . . . . . . p. 146

5 Realizações do protocolo BB84 na presença de interferência . . . . . p. 148

6 Realizações do protocolo BB84 na presença de ruído . . . . . . . . . p. 149

7 Realizações do protocolo BB84 na presença de ruído e interferência · p. 151

8 Execução do algoritmo Cascade . . . . . . . . . . . . . p. 153

9 Realizações do protocolo BB84 na presença de ruído . . . . . . . . p. 155

10 Índices de desempenho de dispositivos de DCQ por fibra óptica . . . . p. 160

11 Índices de desempenho de dispositivos de DCQ por espaço aberto . . p. 160 


\title{
Lista de Abreviaturas e Siglas
}

\author{
ADS Automatic Dependent Surveillance \\ AES Advanced Encryption System
}

AINSC Aeronautical Industry Service Communication

AMSS Amplitude Modulation Signalling System

ATM Air Traffic Management

ATN Aeronautical Telecommunication Network

ATN Aeronautical Telecommunication Network

ATSC Air Traffic Services Communication

BB84 Protocolo criptográfico quântico Bennett-Brassard de 1984

CA Certificate Authority

CIDIN Common ICAO Data Interchange Network

CNS Communications, Navigation and Surveillance

CRL Certificates Revocation Lists

DARPA Defense Advanced Research Projects Agency

DCQ Protocolos de distribuição de chaves quânticas

EPR Estados quânticos propostos inicialmente por Einstein, Podolsky e Rosen EUROCONTROL European Organisation for the Safety of Air Navigation

FAA Federal Aviation Administration 
GSM Global System for Mobile communications

ICAO International Civil Aviation Organization

ICP Infra-estrutura de chaves públicas

IDCQ Infra Estrutura de Distribuição de Chaves Quânticas

ITU-T International Telecommunication Union - Telecommunication Standardization Sector

LFSR linear feedback shift register

Mode S Mode Select

OACA Operating Agency Certificate Authority

OSI Open Systems Interconnection

QBER Quantum Bit Error Rate

RSA Algoritmo criptográfico assimétrico criado por Ron Rivest, Adi Shamir e Leonard Adleman

SIM Subscriber Identity Module

UAT Universal Access Transceivers

WLAN Wireless Local Area Network

X.25 Protocolo do padrão ITU-T para conexão com troca de pacotes em redes em grande áreas 


\section{Lista de Símbolos}

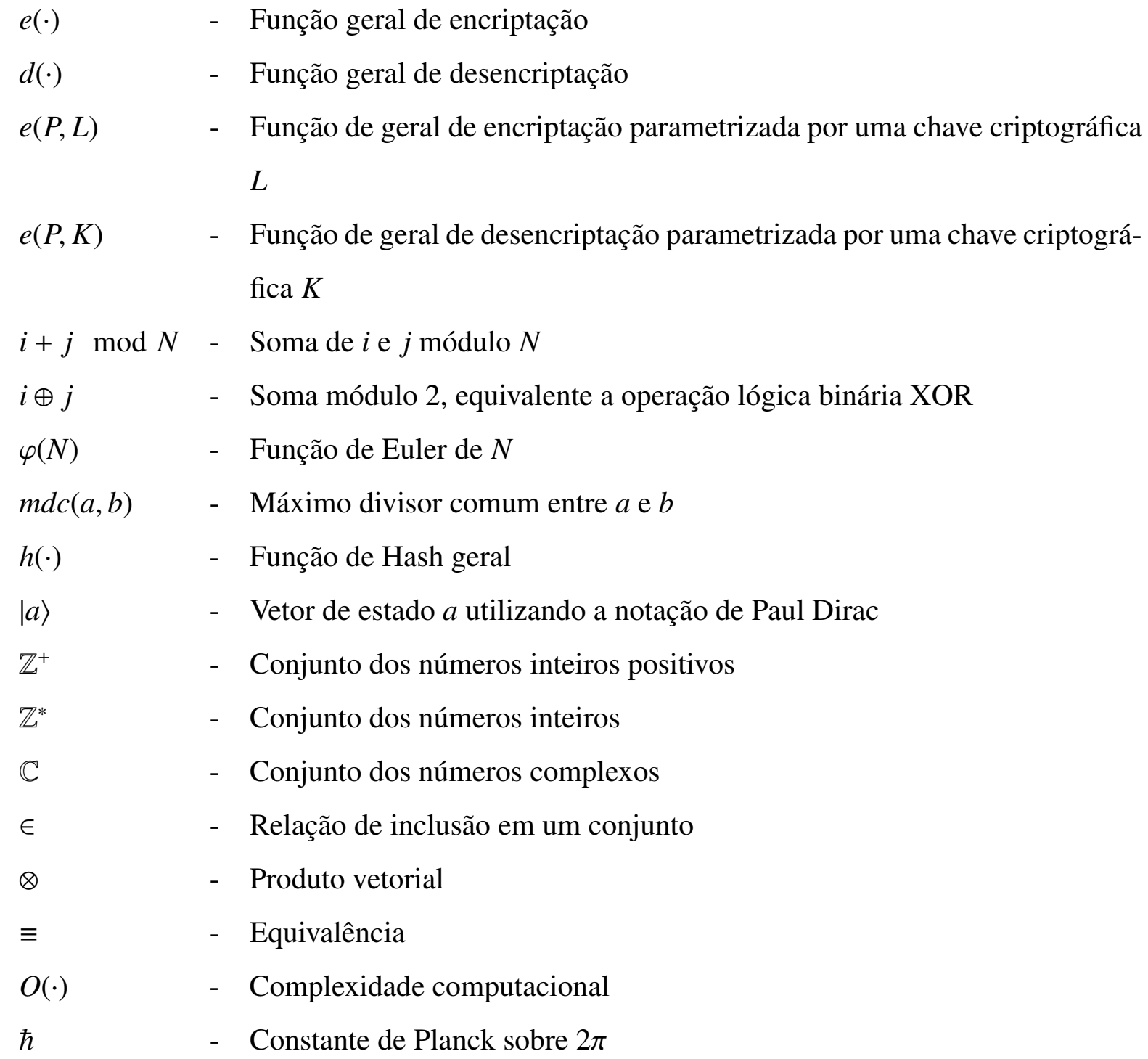




\section{Sumário}

1 Introdução $\quad$ p. 21

1.1 Motivação . . . . . . . . . . . . . . . . p. 21

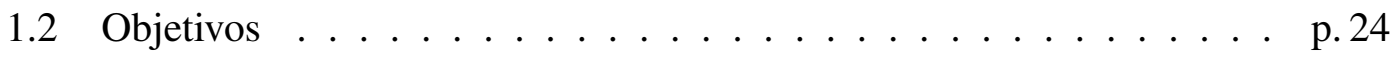

1.3 Organização do trabalho . . . . . . . . . . . . p. 26

2 Criptologia clássica $\quad$ p. 28

2.1 Introdução à criptologia . . . . . . . . . . . . . . . . . . . . . p. 29

2.2 Criptografia de chave simétrica . . . . . . . . . . p. 30

2.2.1 Múltiplos usários . . . . . . . . . . . p. 33

2.2.2 Tamanho e aleatoriedade da chave criptográfica . . . . . . p. 34

2.3 Criptografia de chave assimétrica . . . . . . . . . . p. 36

2.3.1 Algoritmo Diffie-Hellman . . . . . . . . . . . p. 38

2.3.2 Algoritmo RSA . . . . . . . . . . . . . . . . p. 39

2.4 Assinatura digital . . . . . . . . . . . . . p. 43

2.4 .1 Algoritmo ElGamal . . . . . . . . . . . . . p. 44

2.5 Considerações finais do capítulo . . . . . . . . . . . . . p. 46

3 Computação e informação quântica $\quad$ p. 48

3.1 Fundamentos da computação quântica . . . . . . . . . . . . . . . p. 49

3.1.1 Estados emaranhados e múltiplos qubits . . . . . . . p. 53

3.1 .2 Circuitos quânticos . . . . . . . . . . . p. 56 
3.1.3 Paralelismo quântico e o algoritmo de Deutsch . . . . . . . p. 61

3.2 Impacto da computação quântica . . . . . . . . . . . . p. 65

3.2.1 Transformada de Fourier Quântica . . . . . . . . . . . . p. 66

3.2.2 Procedimento de Estimativa de Fase . . . . . . . . . . p. 68

3.2.3 Problema da busca de ordem . . . . . . . . . . p. 70

3.2.4 Como quebrar o RSA com o algoritmo de Shor . . . . . . p. 72

3.3 Considerações finais do capítulo . . . . . . . . . . . . p . 76

4 Criptografia quântica $\quad$ p. 79

4.1 Criptografia quântica . . . . . . . . . . . . p. 80

4.1.1 Protocolos de distribuição de chaves quânticas (DCQ) . . . p. 82

4.1 .2 Protocolo BB84 . . . . . . . . . . . . p. 83

4.1.2.1 Funcionamento do protocolo BB84 na ausência de espionagem ...................... p. 85

4.1.2.2 Protocolo BB84 na tentativa de espionagem . . . p 86

4.1.2.3 Estimativa de erro . . . . . . . . . p. 87

4.1.2.4 Protocolo BB84 em canais com ruído . . . . . . . p. 88

4.1.2.5 Reconciliação de informação . . . . . . . . . . p p. 89

4.1.2.6 Amplificação de privacidade . . . . . . . . p. 92

4.1.3 Ataques contra o protocolo BB84 . . . . . . . . . p. 94

4.1.3.1 Ataque man-in-the-middle . . . . . . . p. 94

4.1.3.2 Interceptação-reenvio . . . . . . . . p. 96

4.1.3.3 Ataque beam-splitting . . . . . . . . p. 97

4.1.3.4 Ataques recentes .............. p. . 98 
4.1.4 Estimativa da informação adquirida por meio de técnicas de

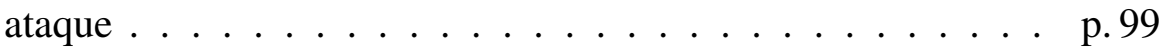

4.1.5 Provas de segurança do protocolo BB84 . . . . . . . . . p. 100

4.1.6 Proteção contra ataques man-in-the-middle . . . . . . . . p. p. 100

4.2 Protocolos quânticos e redes de comunicação . . . . . . . . . . . . . p. 102

4.2.1 Criptografia quântica em comunicações por fibra óptica . . . . p. 104

4.2.2 Criptografia quântica em comunicações por espaço aberto e

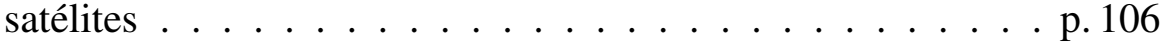

4.3 Considerações finais . . . . . . . . . . . . . . . p. 107

5 Redes de missão crítica: a ATN p. 110

5.1 Visão geral da ATN . . . . . . . . . . . . . . . . . . p. 111

5.1.1 Aplicações da ATN no CNS/ATM . . . . . . . . . . . . p. 113

5.2 Implementação de segurança da informação na ATN . . . . . . . . . p. 114

5.2.1 Mecanismo de gerenciamento de chaves criptográficas . . . p. 116

5.2.2 Segurança de comunicações na ATN utilizando uma ICP . . . p. 117

5.3 Considerações finais do capítulo . . . . . . . . . . . . . . . p. 119

6 Estudo de caso: criptografia quântica em redes aeronáuticas p. 121

6.1 Repetidores quânticos e encaminhadores de dados DCQ . . . . . . . . p. 121

6.2 IDCQ para aplicações A/S . . . . . . . . . . . . . . . p. 123

6.2.1 IDCQ baseada em fonte de fótons no solo . . . . . . . . . . . p. 124

6.2.2 IDCQ baseada em fonte de fótons embarcada em aeronave . . p. 124

6.2.3 IDCQ baseada em fonte de fótons embarcada em satélite . . . p. 125

6.3 IDCQ para aplicações $\mathrm{S} / \mathrm{S} \ldots$. . . . . . . . . . . . p. 127

6.4 Considerações finais do capítulo . . . . . . . . . . . . . p. 130 
7.1 Implementação um protocolo de criptografia quântica . . . . . . . . . p. 134

7.1.1 Definição de parâmetros relevantes . . . . . . . . p. 135

7.1.1.1 Parâmetros do canal quântico . . . . . . . . p. 135

7.1.1.2 Parâmetros Alice: remetente ......... p. 135

7.1.1.3 Parâmetros Bob: destinatário . . . . . . . . p. 136

7.1.1.4 Parâmetros Eva: espião . . . . . . . . . p. 136

7.1.2 Definição da abordagem matemática para simulação . . . . . p. 137

7.1.2.1 Estimativa da informação capturada por Eva . . . . p. 137

7.1.2.2 Simulação do canal quântico . . . . . . . . . p. p. 138

7.1.3 Obtenção de dados aleatórios . . . . . . . . . . . . . p. 139

7.1.3.1 Implementação do colapso de estados . . . . . . . . p. 141

7.1.4 Escopo das funções de implementação . . . . . . . . . . . . p. 141

7.1.4.1 Alice ...................... 141

7.1.4.2 Bob ......................... 143

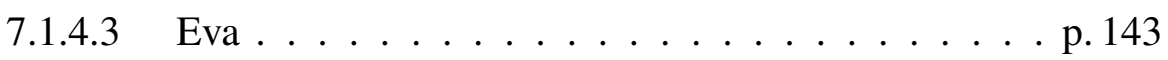

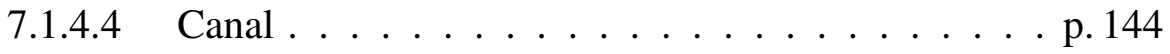

7.1.4.5 Encaminhador quântico . . . . . . . . . p. 144

7.2 Simulação do protocolo BB84 . . . . . . . . . . . . p. 145

7.2.1 Relação entre os parâmetros de simulação e desempenho . . . p. 145

7.2.2 Valores de refência para comparação . . . . . . . . . . p. 159

7.3 Protocolo BB84 em operação conjunta com a ATN . . . . . . . . . p. 162

7.4 Considerações finais do capítulo . . . . . . . . . . . . . . . p. 165 


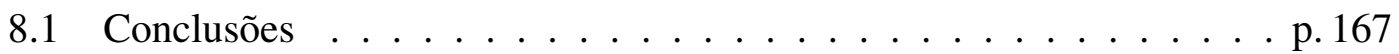

$\begin{array}{lr}\text { Referências } & \text { p. } 172\end{array}$

$\begin{array}{lr}\text { Apêndice A - Requisitos matemáticos } & \text { p. } 177\end{array}$

A.1 Definições . . . . . . . . . . . . . . . . . p. 177

A.2 Algoritmo de Euclides . . . . . . . . . . . . . . . p. 177

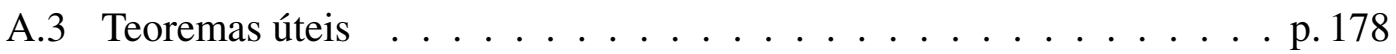

A.4 Conceitos básicos de álgebra linear . . . . . . . . . . . . . . . . p. 179

A.4.1 Espaços vetoriais . . . . . . . . . . . . . p. 179

A.4.2 Bases e independência linear . . . . . . . . . . . p. 180

A.4.3 Auto-vetores e autovalores . . . . . . . . . . p. 180

A.4.4 Operações básicas sobre vetores . . . . . . . . . . . p. 181

A.5 Técnicas de cálculo numérico . . . . . . . . . . . . p. 181

A.5.1 Algoritmo de busca bissectiva . . . . . . . . . . p. 181

Apêndice B - Fundamentos de mecânica quântica p. 183

B.1 Funções de onda e a equação de Schrödinger . . . . . . . . . . . p. 183

B.2 Notação de Dirac . . . . . . . . . . . . . . . . . . . . . p. 184

B.3 Postulados fundamentais da mecânica quântica . . . . . . . . . . . p. 184

B.4 Descrição partícula-onda para a luz . . . . . . . . . . . . . p. 186

B.4.1 Distribuição de Poisson . . . . . . . . . . . . . . . p. 186

B.5 Princípio da incerteza de Heisenberg . . . . . . . . . . . . p. 187

B.6 Teorema da não-clonagem . . . . . . . . . . . . p. 188

$\begin{array}{lr}\text { Apêndice C - Códigos de implementação } & \text { p. } 190\end{array}$

C.1 Escopo das funções de implementação . . . . . . . . . . . . . . . . . p. 190 


\section{$1 \quad$ Introdução}

\subsection{Motivação}

Ocorre atualmente um movimento de aumento da importância que a manutenção de características relacionadas a segurança da informação, como integridade, confidencialidade e autenticidade, vem adquirindo em redes de telecomunicação críticas, movimento este que tem sido acompanhado por uma crescente preocupação envolvendo os fundamentos das técnicas atuais de segurança e as potenciais vulnerabilidades que estas apresentariam com o desenvolvimento de novas e mais eficientes tecnologias de processamento de informação. O impacto que tais fragilidades representariam no modo de utilização de algumas das principais redes de telecomunicações em operação atualmente e a proposição e avaliação de novas técnicas para garantir a segurança da informação em ambientes práticos são os principais motivadores deste trabalho.

Os métodos de manutenção de segurança da informação amplamente utilizados hoje tem sua segurança fundamentada na existência de uma classe de problemas que tem solução difícil de ser obtida computacionalmente. Tais problemas consistem essencialmente de funções em que é fácil obter seu resultado a partir do argumento, mas extremamente difícil obter o argumento a partir do resultado da função. Um exemplo tradicional desse tipo de função é o problema da fatoração. Nesse caso é difícil calcular os fatores primos de um número inteiro grande, mas é fácil testar se dados primos são os fatores de um número inteiro especificado. Contudo, há dois aspectos relevantes sobre a utilização de funções de solução difícil de ser obtidas computacionalmente como mecanismo de segurança para métodos criptográficos.

O primeiro aspecto consiste no fato que para as principais classes de funções utilizadas não há uma prova teórica demonstrando não ser possível obter um mecanismo 
de resolução que consiga encontrar a solução de forma mais eficiente que as técnicas conhecidas. Assim, há uma ausência de garantia que não seja possível obter um método de resolução viável para as funções utilizadas atualmente.

Um segundo aspecto fundamental é representado pelo surgimento de novas tecnologias de processamento de informação, especialmente aquela conhecida como computação quântica. Esse termo se refere aos sistemas computacionais que utilizam propriedades quânticas de sistemas físicos para a representação, processamento e armazenamento de informação, analogamente ao termo convencional computação que refere-se a realização dessas funções por meio de dispositivos eletrônicos clássicos, que utilizam propriedades descritas pela física clássica. Entende-se por sistemas quânticos aqueles sistemas físicos que decorrem de fenômenos naturais em uma escala atômica ou subatômica $\left(\approx 10^{-9} \mathrm{~m}\right)$ e descritos pela bem estabelecida teoria física conhecida como mecânica quântica. As propriedades da mecânica quântica quando aplicadas ao processamento e transmissão da informação geram resultados inalcançáveis para os sistemas atuais, no que se refere a desempenho e segurança. Resultados teóricos e experimentais demonstram que, para algumas classes de problemas computacionais, os computadores quânticos são estritamente mais eficientes, como será apresentado e discutido nos capítulos seguintes. Uma das classes de problemas nos quais os computadores quânticos têm excelentes resultados de desempenho consiste no problema da fatoração de números inteiros. Esse é um fator preocupante quando se considera que esta é a classe de problemas computacionais de difícil solução que é utilizada por um dos algoritmo criptográficos mais importantes atualmente. Esse fato é um indicativo de como a segurança de diversas redes de telecomunicação existentes pode se tornar vulnerável diante de futuras tecnologias de processamento de informação baseadas em sistemas quânticos.

Como consequência da preocupação com a potencial fragilidade dos sistemas criptográficos atuais, algumas propostas de fundamentos alternativos para manutenção da segurança da informação têm sido apontadas. Uma destas consiste na utilização de sistemas quânticos na transmissão de informação, sendo que esta pode ser também bastante beneficiada pelas exclusivas propriedades apresentadas pela mecânica quântica, ocorrendo o surgimento de novas características que aplicadas à transmissão de informação se mostram bastante promissoras. A utilização de fenômenos quânticos na transmissão de informação tem sido demonstrada com precisão crescente e os primeiros dispositivos 
que se beneficiam das novas propriedades quânticas passaram a ser construídos na última década, o que será apresentado e discutido em detalhes ao longo deste trabalho. A principal característica que faz com que a utilização de sistemas quânticos na transmissão de informação seja vantajosa é a existência de limitação física para a ação de leitura e cópia de informação, fazendo com que a informação transmitida por meio desses sistemas seja invariavelmente alterada quando ocorre qualquer tipo de interferência. Essa característica, intrínseca a mecânica quântica, como será mostrado em capítulos posteriores, é bastante útil na construção de técnicas criptográficas e representa o núcleo do que se conhece como criptografia quântica.

A criptografia quântica consiste em um método que utiliza a natureza probabilística da mecânica quântica para a troca de informação com segurança, sendo seu fundamento resistente mesmo na presença de capacidade tecnológica ilimitada, incluindo o cenário com disponibilidade de computação quântica. Desde o primeiro protocolo de criptografia quântica proposto em 1984 por Charles Bennett e Gilles Brassard, conhecido como BB84 e que utiliza a polarização dos fótons de um feixe laser como unidade básica para obtenção de um mecanismo seguro de transmissão de informação, diversas outras abordagens baseadas nesta idéia foram feitas. O fundamento teórico desses protocolos e o sucesso que tem sido obtido na implementação de alguns destes indicam que tal técnica pode ser uma alternativa eficiente para a manutenção da segurança da informação em situações nas quais a segurança é um aspecto crítico.

Há uma classe de redes de telecomunicações em que a vulnerabilidade da informação representa também um risco de segurança física na sua operação. Um bom exemplo de um caso representativo dessa classe é a comunicação aeronáutica, que atualmente, motivada pelos benefícios da comunicação digital, utiliza de forma crescente canais de dados digitais para manutenção de serviços fundamentais para a operação de aeronaves. A consolidação da Aeronautical Telecommunication Network (ATN) como uma rede unificada de troca de informação para o ambiente aeronáutico é um reflexo importante desse processo. Esse é um exemplo de rede em que falhas no âmbito da informação, representadas aqui por acesso indevido, corrompimento de informação, falha de autenticação dentre outras, podem levar a acidentes. A atual implementação da ATN busca satisfazer os requisitos de manutenção da segurança da informação por meio da utilização das técnicas criptográficas amplamente em uso em outras redes, responsáveis pela 
autenticação de mensagens e verificação de autenticidade de dispositivos móveis e fixos na rede. Consideradas as potenciais fragilidades nesses métodos de segurança da informação, o cenário atual gera uma preocupação iminente quanto aos riscos de segurança física que a ATN pode ser submetida.

O atual estágio de desenvolvimento de técnicas de implementação de protocolos de criptografia quântica, no qual, como será visto em detalhes posteriormente, já é possível estabelecer comunicação segura baseada em propriedades quânticas por grandes distâncias e em comunicação por espaço aberto, permite considerar a utilização de criptografia quântica como um mecanismo candidato para a manutenção da segurança da informação em redes de telecomunicações críticas, incluindo o caso de redes aeronáuticas como a ATN.

\subsection{Objetivos}

Este trabalho tem como objetivo analisar a viabilidade da utilização de técnicas de criptografia quântica em infra-estruturas de redes existentes, utilizando como caso de estudo as telecomunicações aeronáuticas por meio da ATN. Para isso são avaliados os protocolos de criptografia quântica existentes e, a partir disso, são desenvolvidos cenários de integração de uma abordagem adaptada de criptografia quântica à redes de telecomunicações aeronáuticas, considerando a infra-estrutura da ATN. A abordagem desenvolvida é então avaliada por meio da obtenção de um ambiente de simulação que permite, juntamente com os dados disponíveis sobre as melhores técnicas de implementação de protocolos quânticos conhecidas e disponíveis, estimar os índices de desempenho esperado para a abordagem proposta. Os índices de desempenho obtidos podem então ser comparados com aqueles verificados nas diferentes canais de comunicação utilizados pela ATN, pelas atuais implementações de segurança da ATN, indicando se a abordagem desenvolvida atende aos requisitos obtidos na operação atual dessa rede. Os resultados deste trabalho contribuem na avaliação da utilização de um protocolo de criptografia quântica em um ambiente de telecomunicações com requisitos realistas assim como na análise da viabilidade da utilização da criptografia quântica em seu estágio atual de desenvolvimento como mecanismo de segurança da informação da ATN, indicando as limitações existentes, propondo novas abordagens no desenvolvimento de 
protocolos quânticos e indicando os possíveis desenvolvimentos nas técnicas de implementação ainda necessários para a integração destes protocolos em casos práticos.

Para alcançar estes objetivos o trabalho se divide em 4 etapas principais. A primeira delas consiste na avaliação das técnicas de criptografia quântica existentes e na escolha de um protocolo que permita a melhor integração a atual estrutura das redes de telecomunicações, considerando a robustez deste diante de técnicas de corrompimento conhecidas, e a maturidade da tecnologia de implementação. A escolha definida é representada por uma adaptação de protocolos e técnicas existentes e algumas novas proposições.

A segunda etapa do trabalho consiste no desenvolvimento de um ambiente de simulação para o protocolo de criptografia quântica definido na primeira etapa. Esta etapa é representada pelo desenvolvimento e implementação de técnicas para simular o comportamentos de sistemas quânticos e da simulação das características de desempenho do protocolo de criptografia quântica desenvolvido.

A terceira etapa do trabalho consiste no levantamento dos requisitos de desempenho requisitados pela atual especificação da ATN e no trabalho de integração da técnica de criptografia quântica desenvolvida às funcionalidades da ATN. Como resultado desta etapa, têm-se a proposição de alguns cenários para a utilização de criptografia quântica no ambiente aeronáutico.

Por fim, na quarta etapa do trabalho é realizada uma estimativa dos índices de desempenho esperados para um implementação física dos cenários propostos. Isso é feito utilizando os dados de desempenho obtidos na simulação do protocolo quântico em conjunto com os dados disponíveis das melhores implementações experimentais conhecidas e divulgadas. Com isso é possível comparar os valores estimados para uma implementação física dos cenários propostos com aqueles encontrados atualmente, permitindo indicar a viabilidade da utilização de protocolos de criptografia quântica nas telecomunicações aeronáuticas e apontar as principais limitações e desenvolvimentos necessários, alcançando o objetivo final do trabalho. 


\subsection{Organização do trabalho}

O trabalho está organizado em 8 capítulos de acordo com o resumo que se segue.

No capítulo 2 são introduzidos os principais conceitos do campo da criptologia, a ciência envolvida na segurança da informação, que serão utilizados no desenvolvimento desse trabalho, a sua terminologia básica e os principais sistemas criptográficos existentes atualmente.

O capítulo 3 tem como objetivo introduzir, utilizando uma abordagem prática, os principais conceitos relacionados a computação quântica e como os resultados obtidos neste campo afetam as ferramentas de criptologia clássica discutidas no capítulo 2 , apresentando como propriedades da mecânica quântica podem ser utilizadas para realizar computação e como as novas propriedades obtidas podem inviabilizar a utilização de algumas técnicas de criptografia clássica.

No caítulo 4 é realizada uma discussão acerca das alternativas disponíveis para sistemas criptográficos na presença de novas tecnologias de processamento de informação e mostrado que não somente a computação como também a transmissão de informação pode ser bastante beneficiada pelas propriedades quânticas, sendo apresentado o conceito de criptografia quântica como uma nova proposta para manutenção da segurança da informação em canais de comunicação. É então feito uma revisão dos principais protocolos de criptografia quântica existentes, discutido algumas de suas vulnerabilidades, maturidade atual de implementação e o adequamento destes a cenários realistas. Por fim o capítulo apresenta alguns parâmetros para a definição do melhor candidato a protocolo de criptografia quântica para utilização na atual estrutura das redes de telecomunicações e apresentadas algumas novas abordagens para a integração destes neste ambiente.

No capítulo 5 é discutido os riscos associados a vulnerabilidades de segurança em redes de missão crítica e apresentada uma visão geral da Aeronautical Telecommunication Network (ATN), seus principais serviços e índices de desempenho obtidos pela implementação autal. É então mostrado os requisitos de segurança da informação presentes na especificação da ATN e como estes requisitos são atendidos na especificação de implementação vigente. Isso leva a discussão sobre o o impacto que vulnerabilidades no âmbito da segurança da informação teria sobre a operação aeronáutica e a 
necessidade do desenvolvimento de novas abordagens para a manutenção da segurança da informação no contexto aeronáutico

O capítulo 6 apresenta o desenvolvimento e proposição de alguns cenários de integração do protocolo de criptografia quântica discutido no capítulo 4 como um mecanismo de segurança operando na estrutura atual da ATN, sendo discutido alguns conceitos fundamentais para a operação prática e adequação da criptografia quântica às aplicações da ATN.

No capítulo 7 se encontra o desenvolvimento da proposta principal deste trabalho representado pela apresentação da abordagem escolhida para a obtenção de um ambiente de simulação para um protocolo quântico e a simulação deste em operação. Com isso e em conjunto com as informações apresentadas nos capítulos anteriores é apresentado o contraste entre os índices alcançados pela abordagem utilizando criptografia quântica e aqueles obtidos pela implementação atual da ATN, indicando a viabilidade da utilização de criptografia quântica no seu estágio atual de implementação e os principais desenvolvimentos ainda necessários.

Por fim, o capítulo 8 apresenta as considerações finais do trabalho juntamente com uma reflexão sobre o grau de atendimentos dos objetivos propostos e os principais impactos decorrentes dos resultados alcançados. Além disso apresenta uma proposta de trabalhos futuros. 


\section{Criptologia clássica}

A manutenção de informação secreta sempre representou um importante papel na história. Diversas situações, como a aquisição do sistema de cifração alemão ENIGMA pelos Aliados na Segunda Guerra Mundial (DENNING, 1982), demonstram que frequentemente o destino de nações inteiras pode depender da habilidade de se decifrar códigos.

O crescente tráfego de informação estimulado pelas novas tecnologias de comunicação e a sensibilidade quanto aos dados trafegados tem feito da ciência de manipular informação secretamente, a criptologia, um conhecimento cada vez mais fundamental em diversas aplicações. Este capítulo tem como objetivo introduzir os principais conceitos desse campo que serão utilizados no desenvolvimento desse trabalho. Para isso é apresentado na seção 2.1 a terminologia básica referente a criptologia. Em seguida, na seção 2.2 são introduzidos os sistemas criptográficos baseados em chaves simétricas e na seção 2.3 são introduzidos os sistemas criptográficos baseados em chaves assimétricas. Por fim, na seção 2.4 é mostrada a obtenção de uma função criptográfica de autenticação, conhecida como assinatura digital, a partir dos métodos criptográficos assimétricos.

O conhecimento obtido do funcionamento das técnicas de segurança apresentados a seguir é utilizado no capítulo 3 para demonstrar como a criptografia atual pode se tornar vulnerável diante de futuras tecnologias de processamento de informação, como a tecnologia conhecida de forma geral como computação quântica. Dessa forma, o termo criptologia clássica será utilizado ao longo do texto para diferenciar as técnicas apresentadas neste capítulo das técnicas de criptologia que serão introduzidas no capítulo 4 , referenciadas como criptografia quântica. 


\subsection{Introdução à criptologia}

A criptologia pode ser divida em duas áreas principais, a criptografia e a criptoanálise. A primeira destas abrange todo esforço de se estabelecer transmissão segura de informação viabilizando diferentes características como confidencialidade, integridade de dados, autenticação e irretratabilidade. A última reúne todas as técnicas que tem como objetivo obter de forma não autorizada mensagens geradas por algum processo de criptografia (MENEZES; OORSCHOT; VANSTONE, 2001).

Como é frequentemente utilizado na literatura sobre criptologia, o conceito de transmissão segura de informação utilizando métodos criptográficos utiliza três personagens principais para ilustrar seu funcionamento: Alice, Bob e Eva. Como padrão, Alice deseja enviar uma mensagem de forma segura a Bob, i.e., prevenindo que Eva acesse a informação original (espionagem) e prevenindo que Eva seja capaz de enviar mensagens a Bob se passando por Alice (personificação).

Para isso Alice realiza o processo de encriptação ou cifração da mensagem original, frequentemente referenciada como mensagem simples, produzindo uma mensagem secreta tal que

$$
E=e_{B}(P)
$$

onde $P$ representa a mensagem simples, $e_{B}(\cdot)$ a função de encriptação de Alice quando se comunicando com Bob e $E$ a mensagem encriptada. Dessa forma, o processo de encriptação é relacionado à codificação da mensagem, enquanto o oposto, a desencriptação, se relaciona a decodificação. Bob recebe então $E$ a partir de um canal de comunicação e por meio da aplicação de uma função de desencriptação correspondente $d_{B}(\cdot)$ obtém a mensagem original, ou seja,

$$
P=d_{B}(E)
$$

A segurança da transmissão da informação nesse caso é mantida pelas funções $e_{B}(\cdot)$ e $d_{B}(\cdot)$, que devem ser mantidas em segredo. Entretanto, essa abordagem não é prática o suficiente porque requer uma grande quantidade de diferentes pares de funções 
para um número típico de usuários em um sistema de comunicação real. Além disso, implementações de hardware ou software de diversas funções distintas é um processo conhecidamente ineficiente.

Uma forma de contornar esse problema é ao invés de manter a função como elemento secreto, utilizar um argumento para uma dada função conhecida por ambos os pares da comunicação e utilizar então este argumento como a informação secreta compartilhada. Esse argumento secreto é referenciado como chave criptográfica em criptografia e faz com que as equações (2.1) e (2.2) se tornem

$$
\begin{aligned}
& E=e\left(P, K_{B}\right), \\
& P=d\left(E, L_{B}\right),
\end{aligned}
$$

onde as funções de encriptação e desencriptação tem como chaves de entrada $K_{B}$ e $L_{B}$ respectivamente e a função utilizada não depende das partes envolvidas na comunicação. Assume-se aqui que as chaves podem ser diferentes com o intuito de garantir a generalidade. Contudo esse fato representará um benefício em apenas alguns casos, o que será discutido em detalhes nas seções seguintes. O diagrama da figura 1 resume as operações desempenhadas por (2.3) e (2.4).

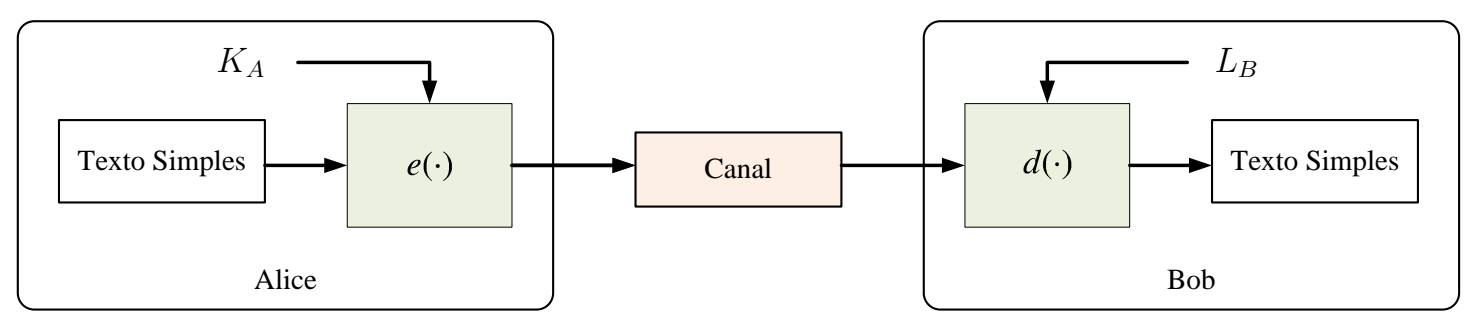

Fig. 1: Conceito básico de transmissão segura de informação

\subsection{Criptografia de chave simétrica}

A situação onde as partes de um sistema de comunicação utilizam a mesma chave criptográfica nos processos de encriptação e desencriptação, i.e. $K_{B} \equiv L_{B}$, é conhecida como criptografia de chave simétrica ou criptografia de chave secreta. Formalmente, a operação de um método geral de criptografia de chave simétrica é definida como na 
Definição 1, retirada de (MENEZES; OORSCHOT; VANSTONE, 2001).

Definição 1. Considerando um esquema de encriptação consistindo de um conjunto de transformações de encriptação e desencriptação $\left\{E_{e}: K \in \mathcal{K}\right\}$ e $\left\{D_{d}: L \in \mathcal{K}\right\}$, respectivamente, onde $\mathcal{K}$ é o espaço de chaves. A encriptação é dita de chave simétrica se para cada par de chaves de encriptação/desencriptação $(K, L)$, é computacionalmente fácil determinar $d(\cdot)$ a partir de e $(\cdot)$ e e $(\cdot)$ a partir de $d(\cdot)$.

Há duas classes de esquemas de criptografia de chave simétrica que são frequentemente distinguidas: cifra de bloco e cifra de fluxo. Os distintos esquemas são definidos como na Definição 2 e Definição 3, respectivamente (MENEZES; OORSCHOT; VANSTONE, 2001).

Definição 2. Uma cifra de bloco é um esquema de encriptação que particiona o texto simples da mensagem original a ser transmitida em blocos de tamanho fixo t sobre um alfabeto $\mathcal{A}$, encriptando um bloco por vez

Definição 3. Seja $\mathcal{K}$ o espaço de chaves para um conjuntos de transformações de encriptação. Uma sequência de símbolos $e_{1} e_{2} e_{3} \ldots e_{i} \in \mathcal{K}$, é chamada de chave de fluxo. Seja $\mathcal{A}$ um alfabeto de q símbolos e seja $E_{e}$ uma cifra de substituição simples com tamanho de bloco igual a 1 onde $e(\cdot) \in \mathcal{K}$. Seja $m_{1} m_{2} m_{3} \ldots$ um texto simples e $e_{1} e_{2} e_{3} \ldots$ uma chave de fluxo de $\mathcal{K}$. Um cifra de fluxo toma o texto simples e produz uma texto cifrado $c_{1} c_{2} c_{3} \ldots$ onde $c_{i}=E_{e i}\left(m_{i}\right)$. Se $d_{i}(\cdot)$ denota o inverso de $e_{i}(\cdot)$, então $D_{d}\left(c_{i}\right)=m_{i}$ realiza a desencriptação do texto cifrado.

Há diversos sistemas criptográficos desenvolvidos utilizando ambas as abordagens. Contudo, não faz parte dos objetivos deste trabalho realizar uma revisão sobre as características dos diversos sistemas criptográficos disponíveis, sendo a referência (MENEZES; OORSCHOT; VANSTONE, 2001) uma boa fonte para isso. Contudo, um sistema de criptografia simétrica merece atenção especial devido a sua utilização posterior neste trabalho, o sistema proposto por Gilbert Vernam conhecido como chave de uso único ou Cifra de Vernam. Tal sistema consiste em um cifra de fluxo definido formalmente na Definição 4 (MENEZES; OORSCHOT; VANSTONE, 2001).

Definição 4. A Cifra de Vernam é uma cifra de fluxo definida sobre o alfabeto $\mathcal{A}=$ 
$\{0,1\}$. Um mensagem binária $m_{1} m_{2} \ldots m_{t}$ é operada por uma chave binária $k_{1} k_{2} \ldots k_{t}$ de mesmo tamanho, produzindo o texto cifrado $c_{1} c_{2} \ldots c_{t}$ onde

$$
c_{i}=m_{i} \oplus k_{i}, 1 \leqslant i \leqslant t .
$$

A grande vantagem da Cifra de Vernam foi provada por Claude Shannon, onde estabelece que se um texto simples e uma chave têm o mesmo tamanho e a chave é verdadeiramente aleatória então o criptossistema é seguro i.e., enquanto as chaves são mantidas secretas então não existe algoritmo sistemático que seja capaz de desencriptar, obter o texto simples, a partir da observação de mensagens encriptadas (SHANNON, 1949).

A Definição 4 pode ser ilustrada por meio da situação: Alice e Bob desejam estabelecer comunicação por meio de um canal seguro. Para isso, eles primeiramente se encontram e definem um par de chaves secretas idênticas comum a ambos. Alice então adiciona aos símbolos da mensagem simples, os bits de uma mensagem digital, os símbolos da chave secreta, sendo a adição módulo $N$, onde $N$ é o tamanho do alfabeto utilizado. Isso produz a mensagem encriptada

$$
E[k]=e\left(P[k], K_{B}\right)=\left(P[k]+K_{B}[k]\right) \bmod N,
$$

onde $E[k]$ refere-se ao $k$-ésimo símbolo da mensagem encriptada e $P[k]$ e $K_{B}[k]$ referemse ao texto simples e a chave secreta respectivamente, i.e., ambos tendo a mesma dimensão $k$. Uma das vantagens deste método reside no fato que o processo de cifragem é bastante simples, o que é fundamental para a diminuição da latência durante o processo de comunicação utilizando-se um sistema de segurança. $\mathrm{O}$ processo de desencriptação é realizado por Bob após receber a mensagem proveniente do canal de comunicação utilizando a operação inversa, ou seja, a subtração módulo $N$. Uma vez que Bob utiliza a mesma chave secreta que Alice, então ele obtém a mensagem simples por meio da operação

$$
P[k]=d\left(E[k], L_{B}\right)=\left(E[K]-L_{B}[k]\right) \quad \bmod N=\left(E[K]-K_{B}[k]\right) \quad \bmod N .
$$


Para o caso binário proposto na Cifra de Vernam tem-se que $N=2$ e as operações de encriptação e desencriptação se tornam iguais i.e. $e(\cdot)=d(\cdot)$.

Como as chaves usadas são mantidas secretas os requisitos definidos anteriormente são atendidos. A despeito de ser um processo simples, a Cifra de Vernam quando utilizada na prática sofre de alguns problemas que podem trazer sérias dificuldades. $\mathrm{O}$ principal deles é o tratamento de um cenário com um grande número de usuários que desejam estabelecer comunicação entre si.

\subsubsection{Criptografia simétrica em um cenário de múltiplos usuários}

A criptografia de chave simétrica tem como requisito que as partes da comunicação entrem em acordo prévio quanto a uma chave comum. Isso não causa sérios problemas quando o número de usuários que desejem se comunicar entre si é pequeno. Contudo, em uma situação prática, tal como com os usuários da Internet ou de uma rede móvel de celular típica (GSM, UMTS, IS95), é inviável a organização do acordo de chaves entre os usuários.

Este problema pode ser contornado de duas formas. A primeira delas é a utilização do conceito de criptografia assimétrica que será introduzido na seção 2.3, que, entretanto, introduz novas vulnerabilidades de segurança como será discutido em detalhes posteriormente. Uma segunda opção é a utilização de diversas chaves estáticas pré-programadas, como é típico nas redes do tipo WLAN. Contudo, como o número de chaves é invariavelmente limitado, então a segurança incondicional não poderá ser alcançada nesse caso, sendo contudo suficiente em grande parte das situações.

Um modelo simplificado da segurança implementada nas redes GSM ajuda a demonstrar a utilização prática da criptografia simétrica em ambientes de telecomunicações reais. Nessa rede cada usuário recebe um chave secreta no momento de sua inclusão. Esta é armazenada no chamado cartão SIM (Subscriber Identity Module) - um tipo especial de smart card localizado no interior do dispositivo móvel - e disponibilizada a cada vez que o usuário inicia uma chamada. Uma cópia fiel da chave de cada usuário é armazenada no Home Location Register (HLR), um repositório digital mantido no operador do sistema. Quando Alice e Bob estão se comunicando a segurança é divida então em duas partes. Cada seção é encriptada entre o usuário e o sistema utilizando a 
chave correspondente, como mostrado na figura 2.

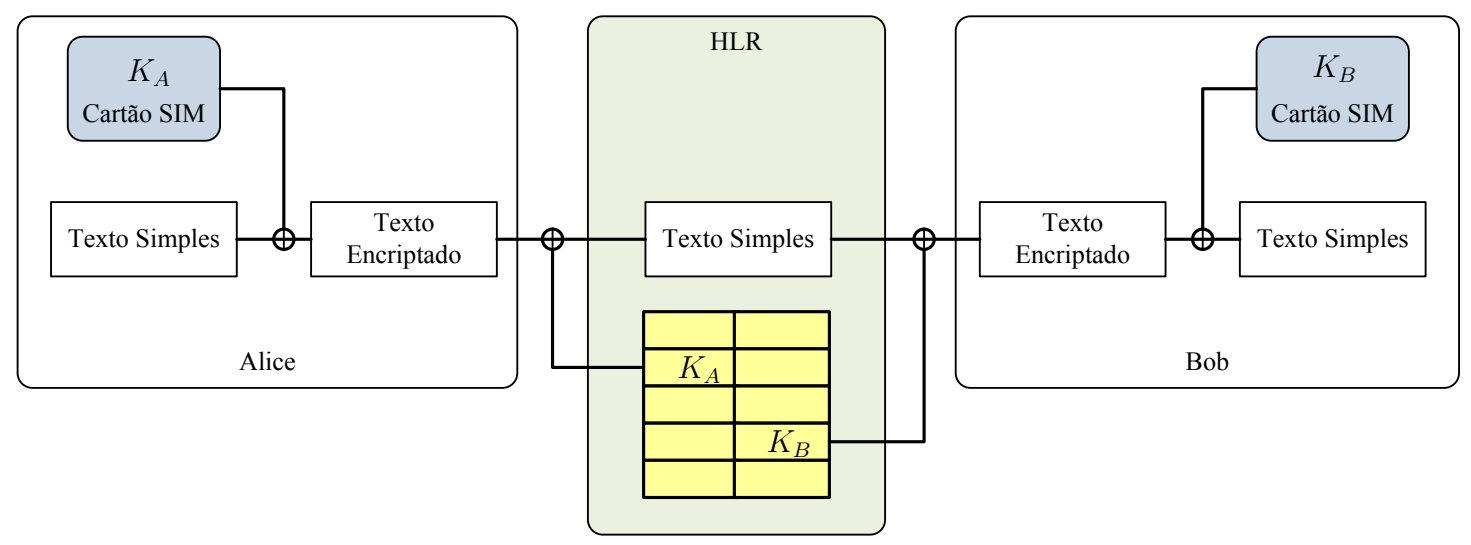

Fig. 2: Sistema criptográfico simétrico em redes móveis públicas

Apesar desse modelo de segurança atender os principais requisitos de segurança existentes neste tipo de comunicação, ao menos dois problemas podem ser revelados. Primeiramente, é evidente que em alguns pontos dentro da rede as mensagens vão trafegar decifradas, o que gera vulnerabilidades nesses pontos. Em segundo lugar vem o fato de que a manutenção do funcionamento dessa rede tem uma infraestrutura estritamente centralizada, o que delega o peso do registro de chaves e gerenciamento a cargo do fornecedor do serviço.

A operação da rede rede GSM é complexa, sendo utilizados diversos algoritmos criptográficos na manutenção da segurança do sistema. A manutenção da privacidade das chamadas é feita pelos algoritmos simétricos de cifra de fluxo A5/1 e A5/2 (MEYER; WETZEL, 2004). Apesar do grande esforço no desenvolvimento destes algoritmos, várias vulnerabilidades têm sido encontradas nos últimos anos em ambos os casos, incluindo a possibilidade de se quebrar a cifra do A5/2 em tempo real (MEYER; WETZEL, 2004). Esse fato evidencia que, excluindo-se o caso da Cifra de Vernam, mesmo algoritmos simétricos bem concebidos estão passíveis de serem corrompidos.

\subsubsection{Tamanho e aleatoriedade da chave criptográfica}

A cifra de Vernam exige a utilização, uma única vez, de uma chave aleatória do tamanho da mensagem para garantir a máxima segurança permitida pelo método. A geração de sequências de números aleatórios pode ser obtida por meio de geradores de números aleatórios desenvolvidos sobre conceitos da teoria da informação ou obtidos de 
medições de características de sistemas físicos (na seção 7.1.3 serão discutidas algumas técnicas de implementação de geradores aleatórios). Contudo, um desafio neste caso é o armazenamento dessas sequências a serem utilizadas como chaves que em casos realistas demanda um grande volume de memória. Sequências menores evitariam custos no armazenamento, contudo implicariam na necessidade de Alice e Bob se encontrem com grande frequência para fazerem o acordo de novas chaves. Ambos os casos são pouco viáveis em ambientes práticos.

Uma técnica de se contornar a dificuldade de se armazenar grandes chaves para a utilização de protocolos de criptografia simétricos como, por exemplo, a Cifra de Vernam, é conhecida como expansão de chave. Essa técnica consiste em expandir uma sequência inicial em uma sequência maior que pode ser usada como chave criptográfica. A segurança da chave expandida depende das características da chave inicial, como por exemplo a existência de algum tipo de padrão de recorrência, e a técnica empregada para realizar a expansão.

Um exemplo frequente da utilização da técnica de expansão de chaves é representada pela implementação física de circuitos ou módulos de software conhecidos como linear feedback shift register (LFSR). O LFSR pode ser utilizado como um gerador de chave (GC) pseudo-aleatória, sendo que este tipo de circuito necessita apenas de uma pequena sequência para ser iniciado. Uma vez alimentado pela sequência de início, o circuito é capaz de gerar uma sequência pseudo-aleatória com longa periodicidade (MENEZES; OORSCHOT; VANSTONE, 2001).

O gerador de chave pode ser utilizado em algoritmos criptográficos simétricos como mecanismo de obtenção de longas chaves criptográficas sem a necessidade de armazenamento ou encontro frequente das parte da comunicação. Com uma pequena chave previamente estabelecida entre Alice e Bob sendo usada como sequência de início do gerador, pode-se produzir uma sequência pseudo-aleatória da dimensão da mensagem a ser trocada, sendo esta sequência utilizada no processo de encriptação (LEWIS; PAYNE, 1973). Neste caso assume-se que o gerador de chave (GC) pode ser incorporado as funções $e(\cdot)$ e $d(\cdot)$.

Devido ao fato de a sequência gerada neste caso não ser verdadeiramente aleatória, há uma violação dos requerimentos de Shannon, não sendo a utilização do sistema de 
Vernam totalmente seguro neste caso. Um parâmetro adicional de segurança nesta situação pode ser a utilização de um vetor de inicialização (VI) que varie no tempo cada vez que uma requisição é feita. O diagrama da figura 3 sintetiza o procedimento. Contudo, não é conhecida nenhuma técnica para se obter um LFSR que produza uma sequência verdadeiramente aleatória a partir de uma pequena sequência inicial.

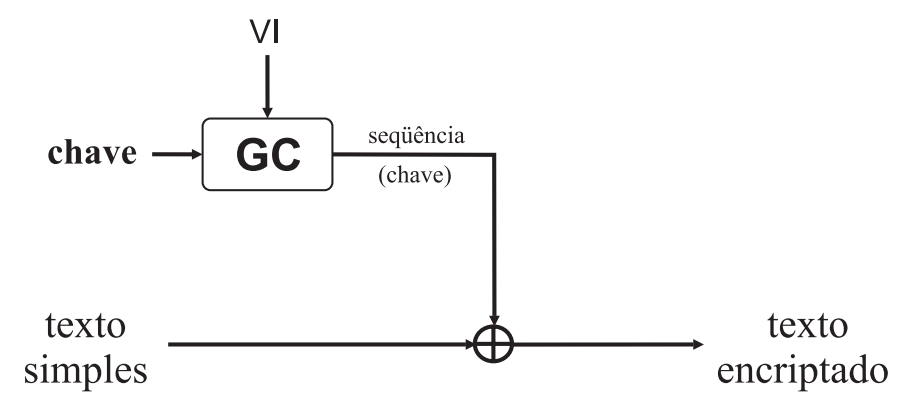

Fig. 3: Encriptação com um gerador de chaves

\subsection{Criptografia de chave assimétrica}

O conceito de um sistema de criptografia de chave simétrica discutido na seção anterior permite o estabelecimento de comunicação segura entre duas partes. Contudo, como requisito para isso é necessário o estabelecimento de uma chave comum entre as partes, mesmo no caso da utilização de um mecanismo de expansão de chaves. Isso implica na necessidade de que as partes da comunicação possuam um meio confiável de trocar informação previamente a comunicação, como o encontro pessoal físico por exemplo. Contudo, sistemas distribuídos como a Internet não permitem o encontro pessoal dos seus usuários, além de não possuírem uma entidade central responsável por atribuir uma chave secreta para cada usuário ou manter um banco de dados para estas.

Um conceito útil nestes casos consiste no que se conhece por criptografia de chave pública ou criptografia assimétrica. Este permite que duas partes em uma comunicação utilizem chaves diferentes no processo de encriptação e desencriptação, respectivamente. A criptografia de chave pública pode ser definida formalmente como na Definição 5 .

Definição 5. Considerando um esquema de encriptação consistindo de um conjunto de funções de encriptação e desencriptação $\left\{E_{e}: e \in \mathcal{K}\right\}$ e $\left\{D_{d}: d \in \mathcal{K}\right\}$, respectivamente. 
O método de encriptação é chamado de um esquema de encriptação de chave pública se para cada par de chaves $(K, L)$, uma chave $K$, chamada de chave pública, é disponibilizada publicamente enquanto a outra L, chamada de chave privada, é mantida em segredo. O sistema é tão seguro quanto a possibilidade de se obter computacionalmente L a partir de $K$.

A definição de um esquema de encriptação de chave pública pode ser entendida de forma prática na comunicação entre Alice e Bob da forma: Bob define sua chave secreta $L_{B}$ para decifrar mensagem provenientes de Alice e anuncia publicamente uma outra chave $K_{B}$ que pode ser usada por Alice para encriptar as mensagens que deseja enviar a Bob. Este recupera a mensagem enviada por Alice por meio de:

$$
d\left(e\left(P, K_{B}\right), L_{B}\right)=P \text {. }
$$

A criptografia de chave publica difere essencialmente do sistema simétrico porque nesta técnica $L_{B} \neq K_{B}$, e, principalmente, as chaves não necessitam ser compartilhadas simultaneamente entre as partes da comunicação.

Além disso, na criptografia de chave pública um requisito adicional deve ser atendido quando se troca a ordem das chaves,

$$
d\left(e\left(P, L_{B}\right), K_{B}\right)=P
$$

ou seja, as chaves devem ser inversas entre si. Essa propriedade é fundamental na obtenção de algumas características importantes dos protocolos assimétricos, como será visto na seção seguinte.

O sistema, como foi proposto anteriormente, permite que um terceiro indivíduo, Eva por exemplo, envie uma mensagem a Bob no lugar de Alice. Isso porque $K_{B}$ é disponibilizada publicamente, sendo acessível a Eva inclusive, que pode então realizar o mesmo processo que Alice. Contudo, a utilização dos pares de chaves pública e privada pode ser aprimorado por meio do adicionamento de novas etapas no processo de encriptação, como mostrado na seção seguinte. 


\subsubsection{Algoritmo Diffie-Hellman}

No algoritmo proposto por Whitfield Diffie e Martin Hellman (DIFFIE; HELLMAN, 1976), o processo de encriptação e desencriptação é realizado em duas etapas e considera que ambas as partes da comunicação, Alice e Bob, possuam seus respectivos pares de chave pública e privada $\left\{L_{A}, K_{A}\right\}$ e $\left\{L_{B}, K_{B}\right\}$. Na primeira etapa Alice realiza uma encriptação sobre o texto simples utilizando sua própria chave privada $L_{A}$. Após isso, Alice realiza nova encriptação com a chave pública de Bob $K_{B}$ sobre o texto cifrado obtido na etapa anterior. Alice obtém então o texto cifrado $E$, tal que:

$$
E=e\left(e\left(P, L_{A}\right), K_{B}\right)
$$

Para recuperar a mensagem original, Bob realiza a operação:

$$
d\left(d\left(E, L_{B}\right), K_{A}\right)=d\left(d\left(e\left(e\left(P, L_{A}\right), K_{B}\right), L_{B}\right), K_{A}\right)=d\left(e\left(P, L_{A}\right), K_{A}\right)=P
$$

que representa uma desencriptação utilizando sua chave privada $L_{B}$ seguida de uma desencriptação utilizando a chave pública de Alice $K_{A}$. Considerando a existência da propriedade mostrada na equação (2.8) para esquemas de encriptação de chave pública, Bob consegue recuperar o texto simples original.

O procedimento realizado por Alice pode ser entendido como um processo de autenticação, o que permite a Bob se certificar que o remetente da mensagem é de fato Alice, impedindo que Eva se comunique com Bob se passando por Alice (DIFFIE; LANDAU, 1998). O diagrama apresentado na figura 4 resume o funcionamento do conceito do algoritmo Diffie-Hellman.

Um aspecto fundamental dos protocolos de criptografia de chave simétrica consiste na obtenção das funções de encriptação e desencriptação, já que a segurança do método se fundamenta na impossibilidade de se obter uma chave privada $L$ a partir do seu par público $K$, de acordo com a Definição 5. O fato de que as funções passíveis de implementação computacional ou física são funções determinísticas, e que por isso são em princípio reversíveis, implica que com a posse de uma chave pública e a função de encriptação/desencriptação seria possível obter a chave secreta sob um ponto de vista teórico. Este fato tornaria os métodos de criptografia de chave pública inúteis. Contudo, 


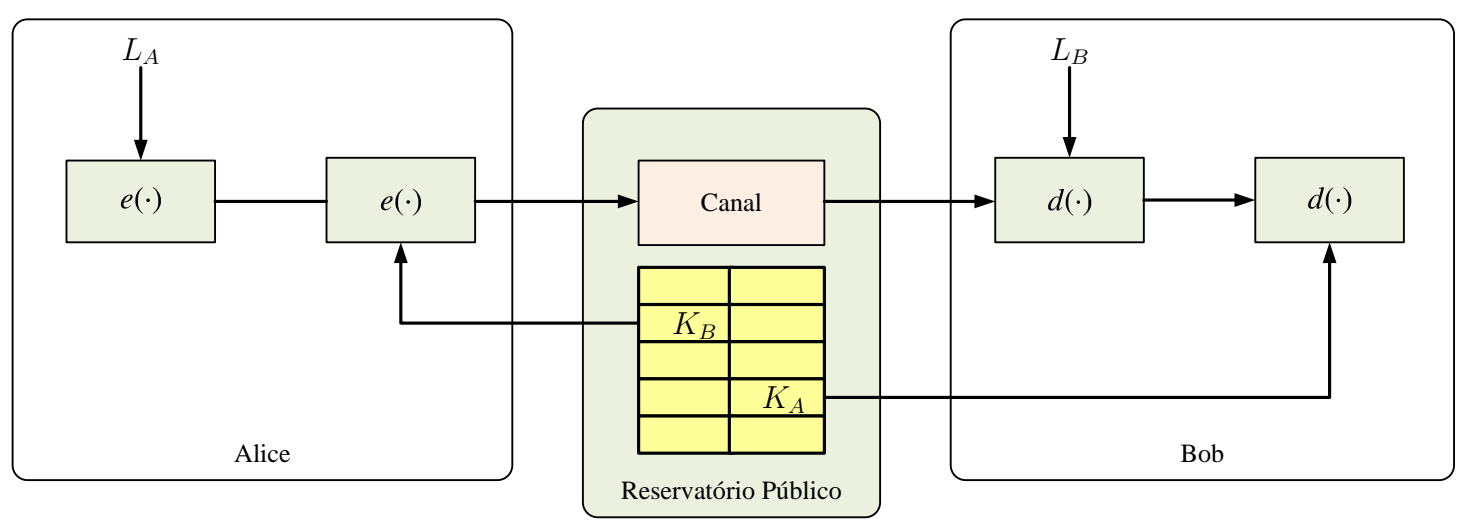

Fig. 4: Esquema de implementação do conceito de Diffie-Hellman

os métodos de chave pública consideram a utilização de uma função de um grupo chamado de one-way functions ou funções de mão-única. Uma função é dita de mão-única se dada $f: x \rightarrow y$, a realização da operação de $f(x)$ é fácil, i.e., rápida, enquanto o processo inverso, calcular $x$ a partir de $y=f(x)$ é muito difícil, i.e., lenta. A existência e a utilização de funções dessa classe atende a Definição 5, e torna os métodos de chave pública tão seguros quanto é difícil reverter uma função de mão-única. Há diversos grupos de funções que têm a propriedade de mão-única. As seções seguintes mostram como algumas funções desta classe são utilizadas na obtenção dos principais algoritmos de criptografia assimétrica disponíveis atualmente.

\subsubsection{Algoritmo RSA}

O algoritmo RSA, sigla obtida a partir das iniciais de seus criadores Ron Rivest, Adi Shamir e Leonard Adleman, é a implementação do conceito de chave pública mais amplamente difundida. Este utiliza a multiplicação como uma função de mão-única. Enquanto a multiplicação de dois inteiros é estritamente simples, encontrar os fatores, i.e., a decomposição em números primos, de um número inteiro grande é um processo difícil, para o qual não existe até o momento nenhum algoritmo computacional eficiente. Um resumo do funcionamento do algoritmo RSA na produção do par de chaves secreta e pública pode ser apresentado da seguinte forma (CORMEN et al., 2003):

1. Bob seleciona aleatoriamente dois números primos grandes $p$ e $q$ tal que $p \neq q$.

2. Calcula-se $N=p \cdot q$. 
3. Bob seleciona aleatoriamente um número ímpar pequeno $a$ de forma que o $m d c(\varphi(N), a)=1$, onde $\varphi(N)$ denota a função de Euler correspondente a $N$ (ver seção A.1). Sendo $N$ o produto de dois primos, do teorema A.3.2 e A.3.5 tem-se $\varphi(N)=\varphi(p) \cdot \varphi(q)=(p-1) \cdot(q-1)$.

4. Em seguida calcula-se o multiplicativo inverso (ver seção A.1) de $a$ no sentido módulo $\varphi(N)$ usando o algoritmo de Euclides (ver seção A.2) e denota-se este por $b:(a \cdot b) \bmod \varphi(N)=1$. Além disso, é garantido a Bob que $b$ existe devido ao teorema A.3.3.

5. Bob anuncia sua chave pública $K_{B}=(a, N)$ e

6. mantém em segredo sua chave privada $L_{B}=(b, N)$.

O processo de encriptação e desencriptação é feito por Bob utilizando as funções

$$
\begin{aligned}
& E=e\left(P, K_{B}\right)=\left(P^{a}\right) \quad \bmod N, \\
& P=d\left(E, L_{B}\right)=\left(E^{b}\right) \bmod N .
\end{aligned}
$$

Utilizando a equação (2.7) é possível demonstrar a aplicação das funções (2.11) e (2.12) no processo de Bob recebendo uma mensagem de Alice utilizando o algoritmo RSA. Da equação (2.7) tem-se que Bob recupera uma mensagem enviada por Alice por meio da operação $d\left(e\left(P, K_{B}\right), L_{B}\right)=P$. Substituindo-se pela chave RSA tem-se então

$$
d\left(e\left(P, K_{B}\right), L_{B}\right)=P=d\left(\left(P^{a}\right) \quad \bmod N, L_{B}\right)=\left(\left(P^{b}\right)^{a}\right) \quad \bmod N=P^{b a} \bmod N
$$

A inversão da ordem das chaves mostra que o algoritmo RSA satisfaz a propriedade de equivalência para o par de chaves pública e privada no processo de encriptação/desencriptação definida na equação (2.8), como pode ser visto por: 


$$
\begin{aligned}
d\left(e\left(P, L_{B}\right), K_{B}\right)=P=d\left(\left(P^{b}\right) \bmod N, K_{B}\right) & =\left(\left(P^{a}\right)^{b}\right) \bmod N \\
& =P^{a b} \bmod N \\
& =P^{b a} \bmod N .
\end{aligned}
$$

A demonstração da validade do algoritmo RSA e de que este permite a recuperação da mensagem original, i.e., que o resultado produzidos nas equações (2.13) e (2.14) correspondem de fato a mensagem original, pode ser feita da forma que se segue.

Demonstração. Explorando o Pequeno Teorema de Fermat (ver A.3.4) e o Teorema de Euler (ver A.3.5), obtém-se que para qualquer $P$ inteiro que é primo relativo a $N$ vale

$$
P^{\varphi(N)} \equiv \bmod N
$$

O teorema A.3.2 mostra que para um número primo $p$ tem-se

$$
\varphi(p)=p-1,
$$

e a partir do teorema do Teorema de Euler decorre que

$$
\varphi(N)=\varphi(p) \cdot \varphi(q)=(p-1) \cdot(q-1)=n-(p+q)+1 .
$$

Desde que $a$ é primo relativo a $\varphi(N), a$ tem então um multiplicativo inverso $b$ tal que:

$$
\begin{aligned}
b \cdot a & \equiv 1 \bmod \varphi(N) \\
& \equiv 1 \bmod (p-1) \cdot(q-1) .
\end{aligned}
$$

Sendo $a$ e $b$ multiplicativos inversos módulo $\varphi(N)=(p-1) \cdot(q-1)$ existe um inteiro $k$ para o qual 


$$
b \cdot a=1+k \cdot \varphi(N),
$$

implicando que

$$
P^{b a} \bmod N \equiv P^{1+k \cdot \varphi(N)} .
$$

A partir da equação (2.15) tem-se que para todo $P$ tal que $p$ não divide $P$ vale

$$
P^{p-1} \bmod p \equiv 1,
$$

e desde que $(p-1)$ divide $\varphi(N)$ tem-se que

$$
P^{k \cdot \varphi(N)+1} \quad \bmod p \equiv P \rightarrow P^{b a} \quad \bmod q \equiv P .
$$

A equação (2.22) é trivialmente verdadeira quando $P \bmod p \equiv 0$, sendo que a igualdade se mantém verdadeira para todo $P$.

Uma argumentação similar produz:

$$
P^{k \cdot \varphi(N)+1} \quad \bmod q \equiv P \rightarrow P^{b a} \quad \bmod p \equiv P .
$$

Com isso, do Teorema Chinês do Resto (teorema A.3.6) e das equações (2.22) e (2.23) é garantido que

$$
P^{b a} \bmod N \equiv P, \forall P .
$$

Dessa forma, o algoritmo RSA permite a obtenção de um par de chaves pública e privada a serem utilizadas no processo de encriptação e desencriptação, garantindo a recuperação da mensagem original após o processo. Nas seções seguintes são discutidas algumas das propriedades do algoritmo RSA e como estas são utilizadas em casos práticos. 


\subsection{Assinatura digital}

O processo de autenticação representado por uma das etapas do algoritmo de DiffieHellman apresentado anteriormente foi utilizado por Alice para prevenir que Eva acesse a mensagem original e para impedir que Eva se passasse por Alice. O procedimento realizado por Alice foi utilizar sua própria chave secreta para cifrar a mensagem que seria enviada a Bob. Esta etapa representa uma funcionalidade criptográfica e pode ser utilizada independentemente da etapa seguinte, sendo conhecida como assinatura digital.

De forma mais detalhada, o procedimento de assinatura digital com ausência de cifragem, i.e., não sendo executado o processo de encriptação que torna o texto nãolegível, garante apenas a autenticação da mensagem e pode ser entendido da forma: Alice produz sua assinatura digital $S$ usando uma função de encriptação (uma função de mão-única, tal como a apresentada no algoritmo RSA), sua chave secreta $L_{A}$ e um texto simples qualquer, da forma:

$$
S=e\left(P, L_{A}\right)
$$

Alice divulga sua chave pública $K_{A}$ e o texto simples $P$. No envio de uma mensagem autenticada ela une a mensagem original com sua assinatura digital pessoal $S$ e envia a Bob. Este por sua vez obtém a chave pública de Alice de um servidor de chaves e o texto simples $P$. Com isso, ele executa o processo inverso sobre $S$ calculando o texto simples presumido

$$
\tilde{P}=d\left(S, K_{A}\right) \text {. }
$$

Por fim, Bob compara $\tilde{P}$ com $P$ e decide se aceita ou rejeita a mensagem recebida como sendo de autoria de Alice. Considerando que a geração da assinatura $S$ a partir de $P$ somente pode ser realizada utilizando-se a chave privada $L_{A}$ de Alice, que é mantida em segredo, e que a função de encriptação utilizada não permite obter $L_{A}$ de $K_{A}$, então se $\tilde{P}$ é igual $P$ Alice é a autora da mensagem. O processo de autenticação por meio de assinatura digital é resumido no diagrama da figura 5. 


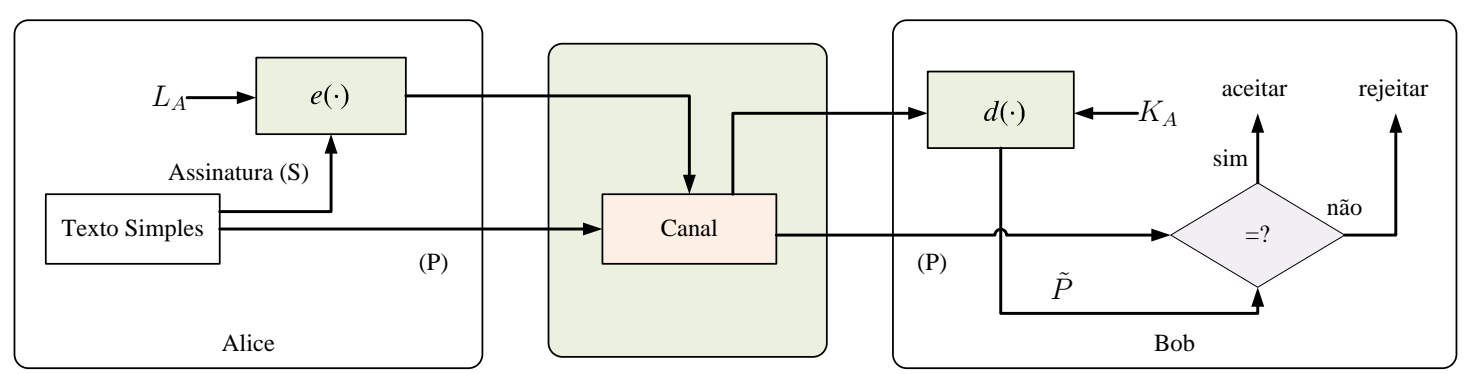

Fig. 5: Arquitetura de implementação do conceito de assinatura digital

\subsubsection{Algoritmo ElGamal}

Um algoritmo de assinatura digital, que é amplamente utilizado, foi proposto por Taher ElGamal e faz uso da teoria de logaritmos discretos para derivar uma função de mão-única. O algoritmo de ElGamal pode ser demonstrado da seguinte forma (ELGAMAL, 1985).

1. Alice escolhe aleatoriamente um número primo grande $p$ e uma gerador $a$ de um grupo multiplicativo $\mathbb{Z}_{p}^{*}$ (ver seção A.1).

2. Em seguida ela escolhe aleatoriamente um inteiro $b$, tal que $1 \leq b \leq p-2$.

3. Alice calcula $y=a^{b} \bmod p \mathrm{e}$

4. anuncia a chave pública $K_{A}=(p, a, y)$ enquanto mantém como chave secreta $L_{A}=b$.

Utilizando suas chaves, Alice gera uma assinatura digital com os passos seguintes.

1. Alice seleciona aleatoriamente um inteiro $l$, tal que $1 \leq l \leq p-2$ e, além disso, $l$ e $(p-1)$ sejam co-primos, i.e., $m d c(l, p-1)=1$.

2. Então ela calcula $r=a^{l} \bmod p \mathrm{e}$

3. o multiplicativo inverso $k$ de $l$ no sentido de módulo $(p-l)$, i.e., $(l \cdot k) \bmod (p-1)$ $=1$.

4. Alice calcula $s=[k \cdot(h(P)-b \cdot r)] \bmod (p-1)$, onde $h(\cdot)$ é chamada função de hash (ver seção A.1), e realiza a transformação conhecida por muitos-para-um de um conjunto de mensagem simples para $\mathbb{Z}_{p}$. 
5. Como passo final, a assinatura de Alice para a mensagem $P$ é $S=(r, s)$.

Com o intuito de verificar o remetente da mensagem Bob realiza o procedimento que segue.

1. Bob obtém a chave pública de Alice $K_{B}=(p, a, y)$ de uma base de dados pública

2. Ele então verifica se $1 \leq r=a^{l} \bmod p \leq p-1$, caso contrário ele rejeita a mensagem.

3. Bob calcula $q_{1}=\left(y^{r} r^{s}\right) \bmod p \mathrm{e}$

4. $h(P)$ e $q_{2}=a^{h(P)} \bmod p$.

5. Por fim, Bob aceita a assinatura como verídica se $q_{1}=q_{2}$.

A demonstração do funcionamento do algoritmo de ElGamal pode ser feita da forma que se segue.

Demonstração. Sabendo que durante o processo de assinatura, Alice produz

$$
s=[k \cdot(h(P)-b \cdot r)] \bmod (p-1) .
$$

Multiplicando-se ambos os lados da equação (2.27) pelo inverso de $k$ obtém

$$
l \cdot s \quad \bmod (p-1) \equiv l \cdot k \cdot(h(P)-b \cdot r)
$$

Sendo $(l \cdot k) \bmod (p-1)=1$, reestruturando a congruência

$$
h(P) \bmod (p-1) \equiv b r+l s,
$$

da qual pode-se concluir que

$$
q_{2}=a^{h(P)} \quad \bmod p \equiv a^{b r+l s} \equiv\left(a^{b}\right)^{r}\left(a^{l}\right)^{s},
$$

utilizando-se o Pequeno Teorema de Fermat (ver A.3.4) tem-se 


$$
a^{h(P)} \bmod p \equiv a^{b r+l s} \equiv\left(a^{b}\right)^{r}\left(a^{l}\right)^{s} \equiv(y)^{r}(r)^{s} \rightarrow q_{2}=q_{1} .
$$

Assim, é possível verificar que o algoritmo de ElGamal permite, tal como proposto, a verificação da autoria da mensagem por meio da utilização de um par de chaves pública e privada. A utilização deste algoritmo em casos práticos será discutida seções seguintes.

\subsection{Considerações finais do capítulo}

As técnicas de criptografia simétrica e assimétrica têm sido amplamente utilizadas nas últimas décadas, tendo um papel fundamental no crescimento das modernas redes de telecomunicações. Ambas as técnicas possuem diversas vantagens e desvantagens entre si, sendo estas complementares em muitos casos. Os algoritmos simétrico têm implementações bastante eficientes, permitindo a operação de encriptação e desencriptação em altas taxas de transferência de dados. Os métodos assimétricos são reconhecidamente mais lentos. Contudo, a necessidade de compartilhamento da chave por ambas as partes no protocolo simétrico representa uma séria dificuldade em casos práticos. No caso assimétrico, o compartilhamento conjunto não é necessário e o método adicionalmente permite, com grande facilidade, a obtenção das primitivas criptográficas de autenticação e assinatura digital (MENEZES; OORSCHOT; VANSTONE, 2001).

Essas características, dentre outras, fazem com que seja bastante frequente a verificação da operação conjunta dos métodos nos sistemas de comunicação atuais. $\mathrm{O}$ algoritmo RSA é amplamente utilizado em redes distribuídas, especialmente a Internet, no estabelecimento do que se conhece como chaves de seção. Estas chaves são então utilizadas em protocolos simétricos, responsáveis pela encriptação das informações transferidas.

Apesar da utilização amplamente disseminada das técnicas de criptografia discutidas anteriormente nas mais diversas redes de telecomunicações, excluindo-se a Cifra de Vernam, todos os métodos conhecidos atualmente têm sua segurança fundamentada 
em premissas não demonstradas teoricamente. Os métodos de criptografia simétricos, especialmente os de cifra de bloco, estão sujeitos ao desenvolvimento de algoritmos de busca exaustiva capazes de pesquisar o espaço de chaves de forma a encontrar a possível chave de forma eficiente explorando alguma vulnerabilidade do método. Os algoritmos assimétricos estão sujeitos ao desenvolvimento de algoritmos mais eficientes que os conhecidos na reversão da função de mão-única utilizada (MENEZES; OORSCHOT; VANSTONE, 2001).

Nos últimos anos, contudo, essas vulnerabilidades não representaram um risco real, uma vez que com os algoritmos conhecidos e a tecnologia disponível, ataques aos métodos criptográficos utilizados eram inviáveis ou muito dispendiosos em grande parte dos casos. Entretanto, o desenvolvimento de novas tecnologias de processamento de informação vem alterando esse cenário. Como será mostrado no capítulo seguinte, o desenvolvimento do que se conhece como computação quântica pode alterar profundamente a utilização dos métodos criptográficos conhecidos hoje. 


\section{Computação e informação quântica}

A tecnologia conhecida como computação quântica têm ganhado na última década espaço crescente na literatura científica especializada e em participação como um dos principais tópicos de grandes orçamentos de pesquisa privada, militar e acadêmica. $\mathrm{O}$ termo computação quântica e/ou informação quântica são os termos gerais para a utilização de sistemas quânticos para a representação, processamento e armazenamento de informação, analogamente ao termo convencional computação que refere-se a realização dessas funções por meio de dispositivos clássicos, ou seja, aqueles descritos pela física clássica (NIELSEN; CHUANG, 2000). Entende-se por sistemas quânticos aqueles sistemas físicos que decorrem de fenômenos naturais em uma escala muito pequena, atômica ou subatômica $\left(\approx 10^{-9} \mathrm{~m}\right)$ e descritos pela bem estabelecida teoria física conhecida como mecânica quântica. Essa teoria representa um grande passo na compreensão dos fenômenos naturais, uma vez que permite uma explicação para diversos efeitos físicos que eram pouco compreendidos com o aparato teórico existente até então. Não obstante, os resultados decorrentes da teoria quântica estão presentes nas principais realizações da indústria eletrônica nos últimos 50 anos.

Há dois fatores principais que motivaram o interesse em utilizar propriedades quânticas para o processamento da informação. O primeiro desses consiste na miniaturização crescente de dispositivos eletrônicos e o surgimento de limitações na descrição teórica utilizada na tecnologia de semicondutores atual, representando talvez a motivação mais pragmática. Contudo, a criação de dispositivos capazes de manipular individualmente sistemas quânticos e, principalmente, o novo paradigma de processamento e transmissão de informação e ganho em eficiência representados pela computação e informação quântica são as principais motivações para o desenvolvimento de novas tecnologias ba- 
seadas em propriedades quânticas. Com isso, este têm sido cada vez mais um tema importante para a engenharia, sendo que esses e outros fatores evidenciam a importância que o estudo dessas propriedades têm sobre o desenvolvimento das novas tecnologias relacionadas à computação e comunicação.

Este capítulo tem como objetivo introduzir os principais conceitos relacionados à computação quântica e informação quântica e como os resultados obtidos neste campo afetam as ferramentas de criptologia clássica apresentadas no capítulo 2. Para isso é apresentado na seção 3.1 como as propriedades da mecânica quântica podem ser utilizadas para realizar computação e na seção 3.2 como as novas propriedades obtidas podem limitar a utilização da criptografia clássica apresentada anteriormente. Os conceitos básicos relacionados a computação quântica discutidos nas seções seguintes são fundamentados principalmente nas referências (NIELSEN; CHUANG, 2000; BOUWMEESTER, 2000; BRYLINSKI; CHEN, 2002; LO; POPESCU; SPILLER, 2001; LOMONACO, 2002).

\subsection{Fundamentos da computação quântica}

Assim como a computação e informação clássica são construídas sobre o conceito fundamental do bit a computação quântica e informação quântica são construídas sobre o conceito do bit quântico ou apenas qubit de agora em diante. Os qubits assim como os bits são independentes de um sistema físico específico, necessitando apenas de um sistema que possa ser descrito pela mecânica quântica, apresentando dessa forma as propriedades que estes elementos utilizam. Dessa forma, os qubits são elementos matemáticos, sendo sua conexão com uma aplicação feita por meio da implementação de alguma técnica capaz de manipular sistemas quânticos.

Utilizando a formulação padrão na mecânica quântica, os qubits podem ser entendidos como vetores de estados pertencentes a um espaço de estados com propriedades apropriadamente definidas (ver apêndice B). Sendo assim, uma notação se torna bastante útil na representação de qubits conhecida como notação de Dirac, que nada mais é do que uma convenção para representação de vetores que facilita a manipulação de algumas propriedades da álgebra linear (ver B.2).

Dessa forma os estados possíveis para um qubit são $|0\rangle$ ou $|1\rangle$, conhecidos como 
base computacional para a computação quântica. A grande diferença da base computacional clássica, na qual os bits assumem os valores 0 e 1 excludentemente, é que os estados escritos na base computacional quântica podem estar em um estado diferente de $|0\rangle$ ou $|1\rangle$. Ocorre que os estados chamados de superposições, que correspondem a combinações lineares dos estados da base computacional, podem ocorrer. Dessa forma, o estado de um qubit pode ser representado por,

$$
|\psi\rangle=\alpha|0\rangle+\beta|1\rangle
$$

sendo $\alpha$ e $\beta$ amplitudes complexas. Isso implica que o qubit é um vetor de um espaço vetorial complexo de duas dimensões, sendo que os estados especiais $|0\rangle$ ou $|1\rangle$ formam uma base ortonormal nesse espaço vetorial (ver seção A.4 para detalhes sobre propriedades da álgebra linear).

Uma função essencial para a computação é a recuperação do valor de um bit. Nesse caso os qubits se comportam de forma essencialmente diferente dos bits clássicos. A mecânica quântica restringe o acesso à informação sobre estados quânticos, fazendo com que a tentativa de medida do estado do qubit a fim de obter os valores de $\alpha$ e $\beta$ seja frustrada. Ao invés disso, a mecânica quântica permite apenas que ao se medir um qubit no estado definido na equação (3.1) seja obtido o estado 0 com probabilidade $|\alpha|^{2}$ e 1 com probabilidade $|\beta|^{2}$. Por se tratarem de probabilidades, os coeficientes devem ter soma igual a 1 ,

$$
|\alpha|^{2}+|\beta|^{2}=1
$$

podendo ser essa condição interpretada geometricamente pela fato do estado de um qubit ser descrito por um vetor normalizado com módulo 1, com o estado geral sendo um vetor unitário em um espaço vetorial complexo de duas dimensões.

Esse comportamento discordante entre a abstração e o mundo tangível existente na mecânica quântica, ao contrário da correspondência direta natural observada com frequência na Natureza, torna difícil a intuição sobre o comportamento de sistemas quânticos. Apesar disso, os estados dos qubits podem ser manipulados e transformados de modo a permitir medidas indiretas, conduzindo a resultados de medidas que 
dependem de diferentes estados. O fato dos estados quânticos terem consequências reais e poderem ser experimentalmente verificados são essenciais para a existência física da computação quântica.

A natureza probabilística de um qubit pode ser exemplificada por uma medição em um dos seus possíveis estados:

$$
|\xi\rangle=\frac{1}{\sqrt{2}}|0\rangle+\frac{1}{\sqrt{2}}|1\rangle .
$$

Para uma medição sobre esse estado se obtém o resultado 0 em $50 \%\left(|1 / \sqrt{2}|^{2}\right)$ das vezes e o resultado 1 em 50\% das vezes. O estado $|\xi\rangle$ da equação (3.3) é recorrente na computação quântica e é frequentemente referenciado por $|+\rangle$. Analogamente existe o estado $|-\rangle$ dado por:

$$
|-\rangle=\frac{1}{\sqrt{2}}|0\rangle-\frac{1}{\sqrt{2}}|1\rangle .
$$

A obtenção de um dos possíveis resultados para uma superposição de estados quânticos é conhecido como colapso de superposição e é um dos postulados fundamentais da mecânica quântica (NIELSEN; CHUANG, 2000) (ver seção B.3 para uma descrição detalhada sobre os postulados fundamentais da mecânica quântica).

Uma representação geométrica para os qubits é aquela em termos de coordenadas esféricas. Como $|\alpha|^{2}+|\beta|^{2}=1$, pode-se escrever o estado de um qubit da forma:

$$
|\psi\rangle=e^{j \gamma} \cos \frac{\theta}{2}|0\rangle+e^{j \varphi} \sin \frac{\theta}{2}|1\rangle,
$$

sendo $\theta, \varphi$ e $\gamma$ números reais e $e^{j \gamma}$ o chamado fator de fase global. Este fator tem módulo igual a 1 e não altera a estatística de uma medição, considerando que esta é feita por meio de uma operação $|\cdot|^{2}$. Sendo assim, os fatores $\theta, \varphi$ definem um ponto sobre uma esfera de raio unitário como aquela mostrada na figura 6 , chamada frequentemente de esfera de Bloch.

A esfera de Bloch permite visualizar que há um número infinito de pontos sobre a esfera, ou seja, um número infinito de superposições em que um qubit pode se manter. Isso motiva uma pergunta: quanta informação pode ser armazenada em um qubit? 


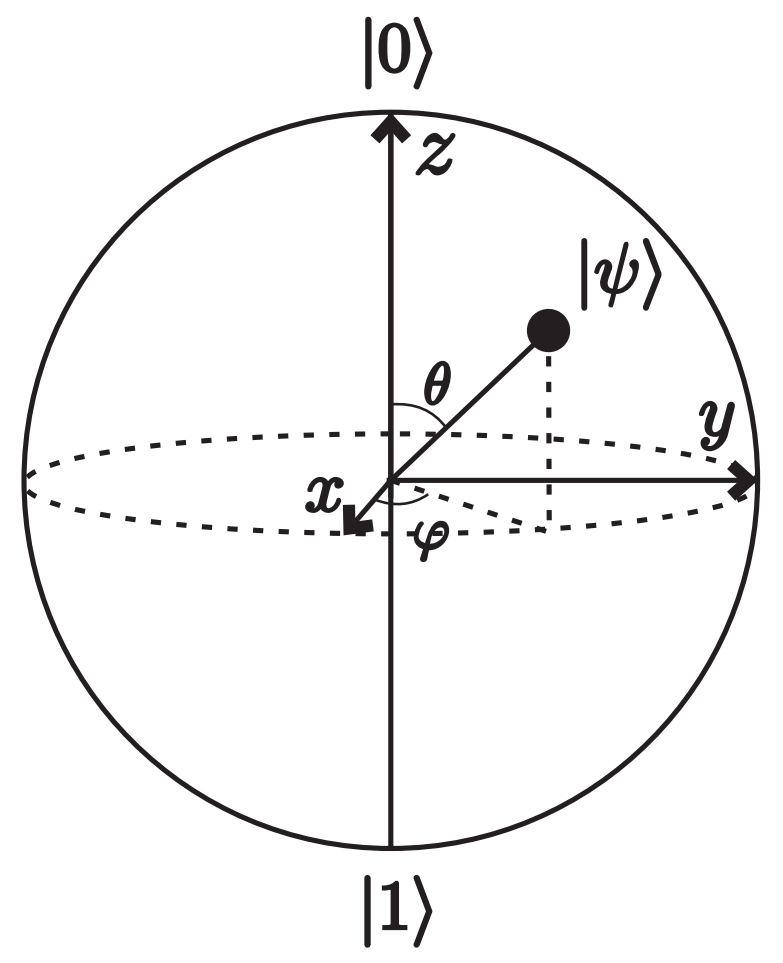

Fig. 6: Representação de um qubit na esfera de Bloch

Estritamente, é possível realizar uma expansão binária infinita em torno de $\theta$, já que $\theta$ é um número real, sendo possível com isso armazenar todo o conhecimento produzido até hoje em um único qubit. Contudo, o fato da realização de uma medida sobre um qubit resultar no colapso de sua superposição para um estado específico faz com que essa conclusão esteja equivocada. Assim, a realização de uma única medida sobre o estado de um qubit resultará em apenas um resultado de dois possíveis, ou um bit clássico. A determinação exata das amplitudes complexas $\alpha$ e $\beta$ poderia ser feita por meio da equação 3.1 para o caso em que fosse possível preparar um número infinito de qubits no mesmo estado. Contudo uma observação importante deve ser feita. A natureza faz um sistema fechado de qubits evoluir sem realizar nenhuma medição, ou seja, ela acompanha todas as variáveis contínuas que descrevem o sistema. Assim, uma grande quantidade de informação oculta é armazena sobre o estado de um qubit. Compreender a natureza dessa informação é o que torna a mecânica quântica uma forma inovadora de manipular informação. 


\subsubsection{Estados emaranhados e múltiplos qubits}

A reunião de diversos qubits também apresenta resultados estritamente distintos daqueles obtidos com registradores clássicos. Para dois bits clássicos há quatro estados possíveis representados por 00, 01, 10 e 11. Para dois qubits os estados da base computacional também são representados por quatro estados: $|00\rangle,|01\rangle,|10\rangle$ e $|11\rangle$. Contudo, os estados da base são neste caso o produto vetorial dador por: $|00\rangle=|0\rangle \otimes|0\rangle$, $|01\rangle=|0\rangle \otimes|1\rangle,|10\rangle=|1\rangle \otimes|0\rangle$ e $|11\rangle=|1\rangle \otimes|1\rangle$, de acordo com a definição algébrica dada para um qubit anteriormente (ver seção A.4 para detalhes sobre propriedades da álgebra linear).

Conhecidamente os qubits podem estar em uma superposição dos estados da base, sendo o vetor de estado que descreve dois qubits escrito como:

$$
|\varphi\rangle=\alpha_{00}|00\rangle+\alpha_{01}|01\rangle+\alpha_{10}|10\rangle+\alpha_{11}|11\rangle
$$

De modo semelhante ao caso anterior de um qubit, uma medição sobre o estado $|\varphi\rangle$ da equação (3.6) resulta em $x(=00,01,10,11)$, com probabilidade $\left|\alpha_{x}\right|^{2}$ de se encontrar $x$ e para os qubits estarem no estado $|x\rangle$ após a medida (ver Postulado (iii) da mecânica quântica na seção B.3). A condição de que a soma das probabilidades de se encontrar todos os resultados possíveis seja 1 é expressa pela condição de normalização:

$$
\sum_{x \in\{0,1\}^{2}}\left|\alpha_{x}\right|^{2}=1
$$

onde $\{0,1\}^{2}$ significa o conjunto de sequências de comprimento dois, com cada letra sendo 0 ou 1.

Como o estado $|\varphi\rangle$ é formado por dois qubits, é possível realizar a medição sobre apenas um dos qubits. Para a medição do primeiro estado, a probabilidade de se encontrar o valor 0 é de $\left|\alpha_{00}\right|^{2}+\left|\alpha_{01}\right|^{2}$, sendo o estado final após a medida dado por:

$$
\left|\varphi^{\prime}\right\rangle=\frac{\alpha_{00}|00\rangle+\alpha_{01}|01\rangle}{\sqrt{\left|\alpha_{00}\right|^{2}+\left|\alpha_{01}\right|^{2}}},
$$

Existe um conjunto de estados de dois qubits que tem um papel fundamental na 
computação quântica e informação quântica. Esses estados foram propostos inicialmente por A. Einstein, B. Podolsky e N. Rosen na referência (EINSTEIN; PODOLSKY; ROSEN, 1935) e ficaram por isso conhecidos como pares EPR ou estados emaranhados. Os resultados foram aprimorados consideravelmente por John Bell e esses pares passaram a serem conhecido também por estados de Bell.

Os estados de Bell são aqueles possíveis para dois qubits da forma

$$
\begin{aligned}
\left|\beta_{00}\right\rangle & =\frac{|00\rangle+|11\rangle}{\sqrt{2}} ; \\
\left|\beta_{01}\right\rangle & =\frac{|01\rangle+|10\rangle}{\sqrt{2}} ; \\
\left|\beta_{10}\right\rangle & =\frac{|00\rangle-|11\rangle}{\sqrt{2}} ; \\
\left|\beta_{11}\right\rangle & =\frac{|01\rangle-|10\rangle}{\sqrt{2}}
\end{aligned}
$$

ou, sintetizando a notação mnemônica anterior pela equação:

$$
\left|\beta_{x y}\right\rangle=\frac{|0, y\rangle+(-1)^{x}|1, \bar{y}\rangle}{\sqrt{2}} .
$$

Esses estados têm a propriedade de, ao se medir o primeiro qubit e se obter um resultado específico, uma medição posterior do segundo qubit sempre terá o mesmo resultado que a primeira medida. Assim, para o caso do par $\beta_{00}$ a medição do primeiro qubit têm probabilidade $1 / 2$ de se obter 0 , levando o estado a $|\xi\rangle=|00\rangle$ nesse caso, e probabilidade $1 / 2$ de se obter 1 , e ainda levando o estado a $|\xi\rangle=|11\rangle$ se isso ocorrer. Isso implica que em uma segunda medição sempre se obterá o mesmo resultado encontrado na primeira. Os estados estão dessa forma correlacionados ou emaranhados, justificando a denominação de estados emaranhados frequentemente utilizada. Essa correlação entre os resultados se mantém para outras medidas, e principalmente, se mantém válida mesmo quando quaisquer outras operações sobre qualquer um dos qubits ocorra. O trabalho de John Bell tem como importante resultado a conclusão que as correlações verificadas em pares EPR são maiores que quaisquer outras que poderiam existir em sistemas clássicos. Esse resultado foi um dos primeiros indícios que a mecânica quântica permitiria o processamento da informação além do que é possível de forma clássica e 
é a chave para algumas das propriedades mais surpreendentes da computação quântica. Os pares de Bell são ainda o protótipo para muitos outras estados quânticos úteis no processamento de informação.

A equação (3.6) mostra que para dois qubits há $2^{2}$ amplitudes complexas que descrevem o estado do sistema. Essa é uma propriedade geral, sendo que para $n$ qubits há $2^{n}$ amplitudes complexas para descrever o estado do sistema. Isso mostra que a informação contida nas amplitudes dos estados dos qubits cresce exponencialmente com o número desses. Considerando um sistema constituído por 500 qubits, haverá então $2^{500}$ amplitudes complexas que descrevem o sistema. Esse número é maior que o número estimado de átomos no universo. Contudo, a natureza é capaz de manipular essa enorme quantidade de dados mesmo em um sistema contendo algumas centenas de átomos. Não há qualquer computador clássico concebível atualmente capaz de armazenar esse volume de informação. Uma metáfora plausível pode ser entendida como se a Natureza mantivesse escondidas $2^{500}$ folhas de rascunho nas quais faz os cálculos à medida que o sistema evolui.

Contudo, a informação contida nas amplitudes dos estados quânticos não é acessível diretamente. Isso porque, como visto anteriormente, ao realizar medições para recuperar os valor dos qubits, o resultado obtido será probabilístico e associado a apenas uma das amplitudes do sistema. A informação contida nas amplitudes dos estados quânticos é por isso conhecida como informação oculta.

A existência da informação oculta e a forte correlação entre os pares de Bell, dentre outras propriedades, dão um indício do potencial de sistemas quânticos no processamento de informação. A natureza probabilística da informação oculta pode, contudo, ser utilizada em sistemas de computação por meio de métodos que manipulem esta informação de forma indireta. Este métodos fazem com que o sistema evolua de uma forma pré-estabelecida, alterando as probabilidades relacionadas às medidas e permitindo que uma medida final retorne o resultado do processamento da informação ocultada nos estados quânticos. Esses métodos são conhecidos como algoritmos quânticos, e os mecanismos que os implementam como circuitos quânticos. O desenvolvimento de algoritmos quânticos capazes de manipular sistemas quânticos a fim de gerar resultados úteis representa um dos principais desafios da computação quântica. As seções seguin- 
tes mostram a teoria básica sobre os circuitos quânticos e como esta pode ser utilizada na obtenção de relevantes algoritmos quânticos.

\subsubsection{Circuitos quânticos}

Os computadores clássicos são construídos por meio de circuitos elétricos que contém vias e portas lógicas. O análogo quântico para processamento e condução de informação quântica é formado por vias e portas quânticas, constituindo os circuitos quânti$\cos$.

As portas quânticas podem agir sobre um único qubit ou sobre vários. Uma porta quântica imediata seria aquela análoga a porta clássica NOT, que leva o bit clássico $0 \rightarrow 1$ e $1 \rightarrow 0$. Espera-se da ação da porta quântica de negação que esta, analogamente ao caso clássico, leve o estado $|0\rangle \rightarrow|1\rangle$ e $|1\rangle \rightarrow|0\rangle$. Além disso, a porta NOT quântica deve agir sobre estados superpostos de forma a inverter os coeficientes, tal que o estado:

$$
|\psi\rangle=\alpha|0\rangle+\beta|1\rangle
$$

torne-se:

$$
|\psi\rangle=\alpha|1\rangle+\beta|0\rangle
$$

A ação da porta NOT quântica deve ser dessa forma linear, característica esta que é geral para todas as portas quânticas, sendo necessária para a manutenção das propriedades algébricas da mecânica quântica (ver apêndice B). Além disso, segue diretamente da linearidade das portas quânticas um modo conveniente de representar a porta quântica NOT. Utiliza-se, por razões históricas, $X$ para a matriz que executa a ação da porta quântica, sendo esta definida da forma:

$$
X \equiv\left[\begin{array}{ll}
0 & 1 \\
1 & 0
\end{array}\right]
$$

Escrevendo o estado da equação (3.14) de forma vetorial: 


$$
|\psi\rangle=\alpha|0\rangle+\beta|1\rangle=\alpha\left[\begin{array}{l}
1 \\
0
\end{array}\right]+\beta\left[\begin{array}{l}
0 \\
1
\end{array}\right]=\left[\begin{array}{l}
\alpha \\
\beta
\end{array}\right],
$$

com a linha de cima correspondendo a amplitude de $|0\rangle$ e a linha de baixo a amplitude de $|1\rangle$. A ação da porta quântica NOT no vetor de (3.17) resulta em:

$$
X\left[\begin{array}{l}
\alpha \\
\beta
\end{array}\right]=\left[\begin{array}{l}
\beta \\
\alpha
\end{array}\right]
$$

A ação de portas quânticas sobre um qubit são definidas por matrizes $2 \times 2$ que têm como restrição que o estado resultante de sua ação mantenha válida a condição de normalização definida na equação 3.20. Assim, dada a ação de uma porta arbitrária $U$ tal que:

$$
|\psi\rangle=\alpha|0\rangle+\beta|1\rangle \stackrel{U}{\rightarrow}\left|\psi^{\prime}\right\rangle=\alpha^{\prime}|0\rangle+\beta^{\prime}|1\rangle
$$

a relação de normalização deve se manter para:

$$
\left|\alpha^{\prime}\right|^{2}+\left|\beta^{\prime}\right|^{2}=1
$$

Essa condição implica no fato que a matriz que representa a ação da porta quântica seja unitária, o que significa que:

$$
U^{\dagger} U=I
$$

sendo $U^{\dagger}$ a conjugada transposta de $U$, ou apenas adjunta de $U$, e $I$ a matriz identidade. De forma mais geral, a restrição da ação de portas serem representadas por matrizes unitárias é na verdade a única restrição sobre qualquer porta quântica, tornando o projeto dessas bastante flexível.

Além da porta $X$, mais duas portas quânticas bastante úteis podem ser derivadas das matrizes de Pauli (COHEN-TANNOUDJi; DUI; LALOE, 1978). Dessa forma, tem-se que: 


$$
\begin{aligned}
& Y \equiv\left[\begin{array}{cc}
0 & -i \\
i & 0
\end{array}\right], \\
& Z \equiv\left[\begin{array}{cc}
1 & 0 \\
0 & -1
\end{array}\right] .
\end{aligned}
$$

Outras portas quânticas que agem sobre um qubit são as portas de Hadamard, porta de fase e porta $\pi / 8$, definidas respectivamente pelas matrizes

$$
\begin{gathered}
H \equiv \frac{1}{\sqrt{2}}\left[\begin{array}{cc}
1 & 1 \\
1 & -1
\end{array}\right], \\
S \equiv\left[\begin{array}{ll}
1 & 0 \\
0 & i
\end{array}\right], \\
T \equiv\left[\begin{array}{cc}
1 & 0 \\
0 & e^{i \pi / 4}
\end{array}\right]
\end{gathered}
$$

A porta de Hadamard, conhecida como raiz quadrada de NOT, transforma $|0\rangle$ em $(|0\rangle+|1\rangle) \sqrt{2}$ e $|1\rangle$ em $(|0\rangle-|1\rangle) \sqrt{2}$. Essa porta é bastante útil na computação quântica, sendo utilizada nos principais circuitos quânticos conhecidos, como será visto nas próximas seções. A diagramação mais utilizada para circuitos quânticos é semelhante a diagramação de circuitos clássicos. Assim, os circuitos quânticos são lidos da esquerda para direita, onde as portas representam a execução de uma dada operação sobre os estados quânticos. Algumas portas de um qubit são mostradas na figura 7 em comparação a ação da porta clássica NOT.

As portas quânticas podem ser generalizadas para a ação em vários qubits. Em circuitos clássicos a reunião das portas AND e NOT clássicas, ou apenas NAND, é reconhecida como porta universal a medida que é possível reproduzir a ação de qualquer outra porta clássica por meio dessa. Os circuitos quânticos tem o análogo representado pela porta quântica de dois qubits NOT-conTrolada (Controlled-NOT), ou apenas CNOT. A porta tem como entrada dois qubits, o qubit de controle e o qubit alvo, sendo sua ação condicionada pelo qubit de controle. No caso deste estar no estado $|0\rangle$ nada 


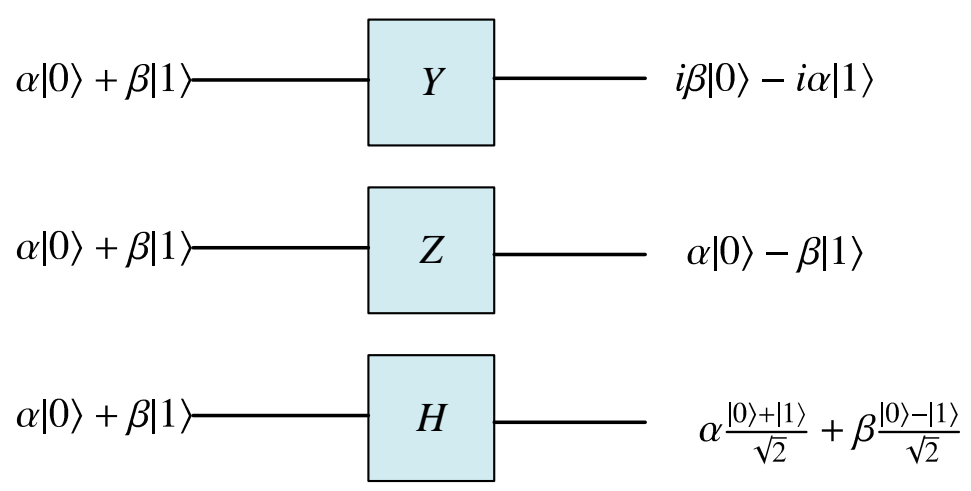

Fig. 7: Porta clássica de um bit à esquerda e portas quânticas de um qubit à direita acontece com o qubit alvo, ao passo que este estando no estado $|1\rangle$ o qubit alvo tem seu estado trocado. Assim, ação da porta CNOT sobre dois qubits produz os resultados:

$$
|00\rangle \rightarrow|00\rangle ;|01\rangle \rightarrow|01\rangle ;|10\rangle \rightarrow|11\rangle ;|11\rangle \rightarrow|10\rangle
$$

De forma resumida a operação CNOT pode ser escrita da forma

$$
\text { CNOT }:|x\rangle|y\rangle \rightarrow|x\rangle|x \oplus y\rangle
$$

onde $x$ e $y$ representam os valores clássicos para o bit, i.e., 0 e 1 .

A operação lógica quântica CNOT é definida por uma matriz 4x4 da forma:

$$
U_{C N} \equiv\left[\begin{array}{cccc}
1 & 0 & 0 & 0 \\
0 & 1 & 0 & 0 \\
0 & 0 & 0 & 1 \\
0 & 0 & 1 & 0
\end{array}\right],
$$

sendo representa pelo diagrama da figura 8 .

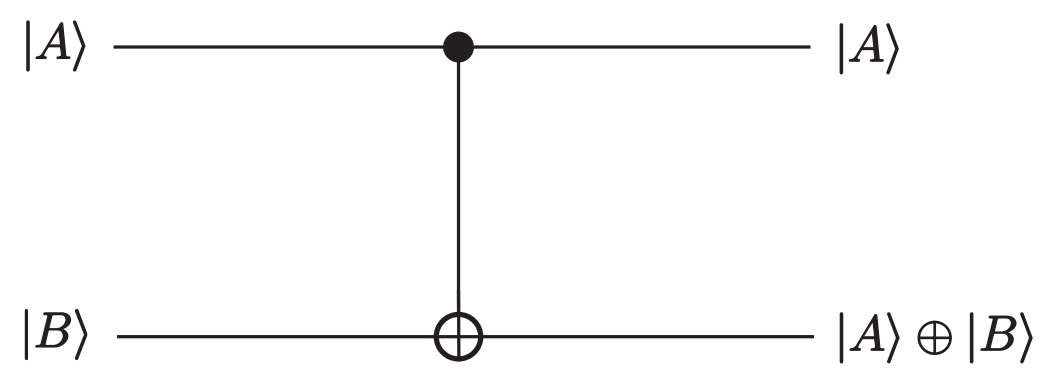

Fig. 8: Porta quântica CNOT 
No diagrama da figura $8,|A\rangle$ é o qubit de controle e $|B\rangle$ o qubit alvo. A notação utilizada é a mais recorrente na literatura para representar a ação da porta CNOT para dois qubits.

A figura 9 mostra como as portas quânticas CNOT e Hadamard podem ser utilizadas para a obtenção de um circuito gerador dos pares de Bell apresentados na seção anterior.

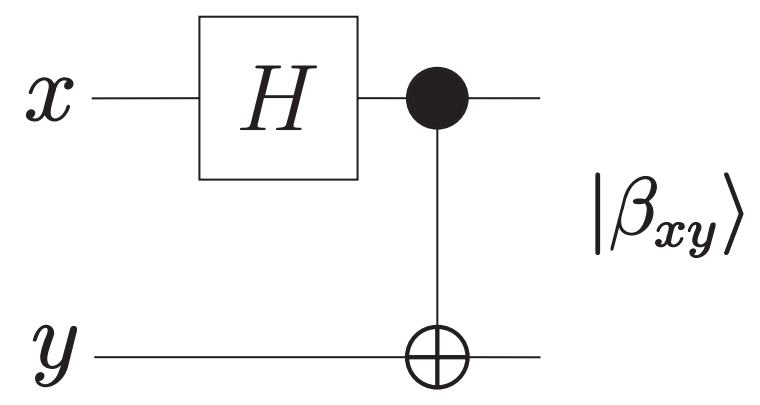

Fig. 9: Circuito quântico para criar estados de Bell

Uma consequência importante da única restrição imposta a definição das portas quânticas, a de serem matrizes unitárias, é que devido a isso essas portas são, ao contrários das portas clássicas, sempre reversíveis. As portas clássicas universais XOR e NAND são essencialmente irreversíveis ou não-reverssíveis. O resultado da operação $A \oplus B$ da porta XOR não permite determinar quais foram as entradas $A$ e $B$, fazendo com que ocorra uma perda irrecuperável de informação com a ação da porta. Considerando a propriedade que é sempre possível obter a matriz inversa de uma matriz unitária, sendo esta também unitária, as portas quânticas podem então sempre ter suas ações revertidas. Compreender como realizar operações utilizando a lógica reversível é o passo crucial para tirar proveito das propriedades quânticas que conferem grande poder computacional aos sistemas quânticos.

Uma questão tecnológica relevante é avaliar a possibilidade de simular os circuitos lógicos clássicos com circuitos quânticos. A resposta para essa questão é positiva e isso é feito por meio da porta quântica reversível chamada de porta de Toffoli. Esta porta tem como entrada 3 qubits, sendo que dois desses são para controle não sendo afetados pela ação da porta. O terceiro qubit é o alvo e tem seu estado alterado apenas no caso dos dois qubits de controle estiverem no estado |1 $\rangle$. A ação da porta de Toffoli em um dado conjunto de bits consiste em $(a, b, c) \rightarrow(a, b, c \oplus a b) \rightarrow(a, b, c)$, o que mostra a reversibilidade da porta. 
A porta de Toffoli pode ser utiliza para simular a ação da porta clássica NAND, o que pode ser deduzido a partir de sua tabela-verdade:

Tab. 1: Tabela-verdade da porta de Toffoli

\begin{tabular}{cccccc}
\hline$a$ & $b$ & $c$ & $a^{\prime}$ & $b^{\prime}$ & $c^{\prime}$ \\
\hline 0 & 0 & 0 & 0 & 0 & 0 \\
$\mathbf{0}$ & $\mathbf{0}$ & $\mathbf{1}$ & $\mathbf{0}$ & $\mathbf{0}$ & $\mathbf{1}$ \\
0 & 1 & 0 & 0 & 1 & 0 \\
$\mathbf{0}$ & $\mathbf{1}$ & $\mathbf{1}$ & $\mathbf{0}$ & $\mathbf{1}$ & $\mathbf{1}$ \\
1 & 0 & 0 & 1 & 0 & 0 \\
$\mathbf{1}$ & $\mathbf{0}$ & $\mathbf{1}$ & $\mathbf{1}$ & $\mathbf{0}$ & $\mathbf{1}$ \\
1 & 1 & 0 & 1 & 1 & 1 \\
$\mathbf{1}$ & $\mathbf{1}$ & $\mathbf{1}$ & $\mathbf{1}$ & $\mathbf{1}$ & $\mathbf{0}$ \\
\hline
\end{tabular}

As linhas em negrito da tabela 1 evidenciam que a ação da porta é idêntica a ação da porta NAND quando a entrada $c$ está em 1 , ou seja, $(a, b, 1) \rightarrow(a, b, 1 \oplus a b)=$ $(a, b, \operatorname{not}(a b))$. O diagrama da porta de Toffoli e a situação em que esta se comporta como uma porta clássica NAND são mostrados na figura 10. Essa operação implica que é possível simular todas as ações das portas clássicas convencionais, e portanto, qualquer circuito clássico completo pode ser simulado por meio de circuitos quânticos reversíveis equivalentes.

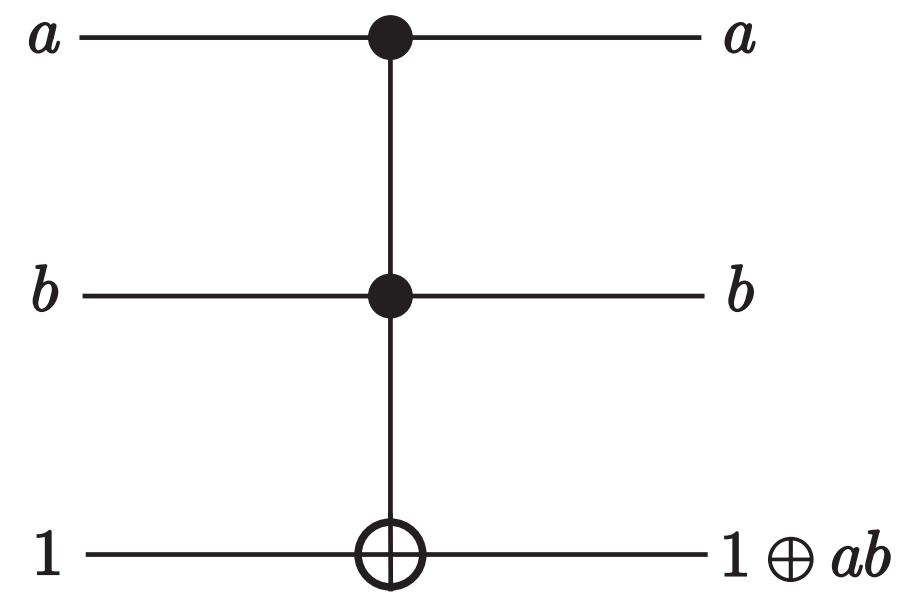

Fig. 10: Representação diagramática da porta de Toffoli

\subsubsection{Paralelismo quântico e o algoritmo de Deutsch}

Nesta seção é mostrado como, utilizando as portas quânticos apresentadas anteriormente, é possível obter circuitos que implementam algoritmos quânticos capazes de fazer uso prático da informação ocultada nos estados quânticos. 
Uma utilização prática da informação oculta é evidenciada por meio do algoritmo de Deutsch, um algoritmo quântico que utiliza a propriedade geral de circuitos quânticos conhecida como paralelismo quântico. Essa propriedade se refere à capacidade que circuitos quânticos têm em avaliar funções matemáticas para muitos argumentos de valores diferentes. A utilização do paralelismo quântico é mostrada na construção do algoritmo de Deutsch como se segue.

Dada uma função desconhecida $f(x):\{0,1\}^{n} \rightarrow\{0,1\}$, a forma clássica de se determinar a regra exata de operação dessa função é substituir todas as entradas possíveis $x=0,1, \ldots, 2^{n}-1$, o que requer ou um grande número de avaliações para $f(x)$ ou a utilização de $N=2^{n}$ portas clássicas elementares implementando $f$.

O tratamento desse problema utilizando-se circuitos quânticos pode ser feito com apenas um passo por meio do algoritmo de Deutsch. A obtenção do algoritmo pode ser apresentada de forma resumida da seguinte forma: primeiro é mostrada a solução para o problema da função $f(x):\{0,1\}^{1} \rightarrow\{0,1\}^{1}$ e posteriormente a generalização para a função $f(x):\{0,1\}^{n} \rightarrow\{0,1\}^{1}$.

Inicialmente considera-se a porta CNOT apresentada anteriormente e a ação executada por esta sobre os qubits de entrada. Para o qubit de controle $|C\rangle_{I N}=\frac{|0\rangle+|1\rangle}{\sqrt{2}}$ e o qubit alvo $|D\rangle_{I N}=|0\rangle$, tem-se como resultado a produção do par de Bell $\frac{|00\rangle+|11\rangle}{\sqrt{2}}$.

Das propriedades discutidas para os pares de Bell na seção 3.1.1 percebe-se que os dados de saída contém ambas as possibilidades de entrada. A expressão para a porta CNOT apresentada na equação (3.28) pode ser modificada e feita a definição de uma transformação $U_{f}$ tal que:

$$
U_{f}:|x\rangle|y\rangle \rightarrow|x\rangle|y \oplus f(x)\rangle
$$

com $x$ e $y$ sendo os valores possíveis para bits clássicos. A figura 11 apresenta um diagrama do circuito proposto, enquanto a tabela 2 consiste na tabela-verdade para o circuito.

Assumindo então as mesmas entradas para a porta CNOT e considerando a tabela 2, é fácil calcular a saída da porta CNOT sendo controlada pela função $f$ : 


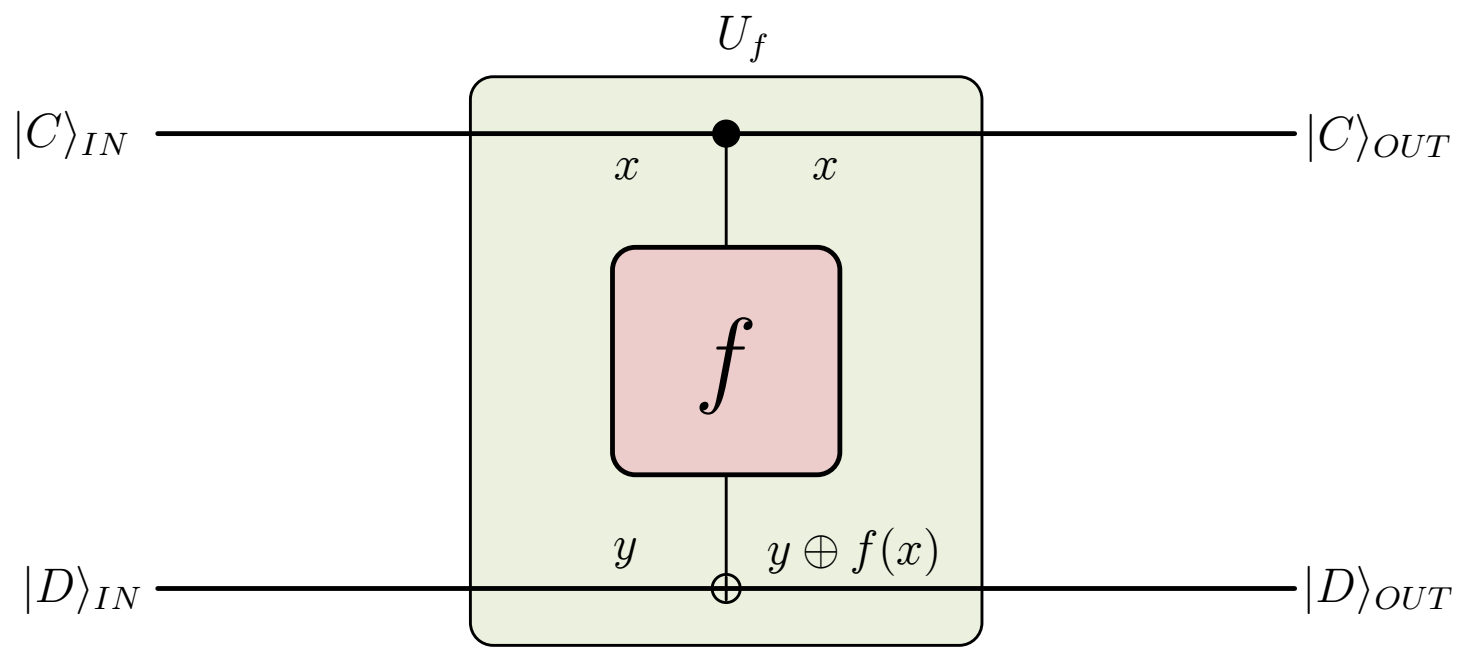

Fig. 11: Circuito para implementação do algoritmo de Deutsch

Tab. 2: Tabela-verdade da porta CNOT controlada por $f$

$$
\begin{array}{cc|cl}
\hline x & y & x & y \oplus f(x) \\
\hline 0 & 0 & 0 & 0 \oplus f(0)=f(0) \\
0 & 1 & 0 & 1 \oplus f(0) \\
1 & 0 & 1 & 0 \oplus f(1)=f(1) \\
1 & 1 & 1 & 1 \oplus f(1) \\
\hline & \\
U_{f} \frac{|00\rangle+|10\rangle}{\sqrt{2}}=\frac{|0\rangle|f(0)\rangle+|1\rangle|f(1)\rangle}{\sqrt{2}} .
\end{array}
$$

A equanção (3.31) contém ambos os valores possíveis de $f$, ou seja, $f(0)$ e $f(1)$, após uma única iteração do circuito. Devido às propriedades quânticas, a entrada $|C\rangle_{I N}$ possui $|0\rangle$ e $|1\rangle$, i.e., todos os valores possíveis de $x$.

A porta de Hadamard apresentada anteriormente tem a propriedade de gerar todas as superposições possíveis para $x$, com tamanho de $n$ bits, se esta for alimentada com o estado inicial $\left|0 \ldots 0^{2^{n}}\right\rangle$. Esse resultado permite generalizar aquele encontrado na equação (3.31) para o caso de $n$ bits, utilizando para isso o resultado da porta de Hadamard de dimensão $n$ como controle para a porta CNOT controlada por $f$. Dessa forma a equação (3.30) pode ser reescrita da forma:

$$
U_{f}:|x\rangle_{n}|y\rangle \rightarrow|x\rangle_{n}|y \oplus f(x)\rangle
$$

Com isso o resultado do circuito é dado por: 


$$
\begin{aligned}
U_{f} \frac{1}{\sqrt{2^{n}}} \sum_{x \in\{0,1\}^{n}}|x\rangle|0\rangle & =\frac{1}{\sqrt{2^{n}}} \sum_{x \in\{0,1\}^{n}}|x\rangle|0 \oplus f(x)\rangle \\
& =\frac{1}{\sqrt{2^{n}}} \sum_{x \in\{0,1\}^{n}}|x\rangle|f(x)\rangle \\
& =\frac{1}{\sqrt{2^{n}}} \sum_{x=0}^{2^{n}-1}|x\rangle|f(x)\rangle .
\end{aligned}
$$

Isso implica que o circuito tem a capacidade de determinar todas as correspondências de $x$ e $f(x)$ avaliando a função apenas uma única vez. Um computador probabilístico clássico, aparato atualmente utilizado para executar esse tipo de tarefa, pode avaliar $f(0)$ com probabilidade $1 / 2$ ou $f(1)$ com probabilidade $1 / 2$, sempre mutuamente excludente. $\mathrm{O}$ algoritmo quântico de Deutsch é então mais rápido que qualquer aparato clássico.

A essência do projeto de algoritmos quânticos está na escolha inteligente da função e da transformação final que determinam as propriedades globais das funções. $\mathrm{O}$ algoritmo de Deutsch sugere que computadores quânticos podem resolver alguns problemas computacionais com eficiência maior que computadores clássicos. Infelizmente o algoritmo de Deutsh é de pouco interesse prático, mas o resultado alcançado por este motivou o interesse pelo desenvolvimento de algoritmos quânticos, resultando em diversos algoritmos de uso prático. Não faz parte do escopo deste trabalho realizar uma revisão detalhada dos algoritmos quânticos conhecidos, sendo a referência (NIELSEN; CHUANG, 2000) um bom compêndio dos principais algoritmos quânticos disponíveis e os problemas que estes tratam. Há, contudo, três classes de problemas que são conhecidamente mais eficientes em computadores quânticos:

1. Algoritmos baseados na versão quântica da Transformada de Fourier: Estimativa de Fase, Busca de ordem, Fatoração de Shor, solução do logaritmo discreto e solução do problema do subgrupo oculto;

2. Algoritmos de busca: Grover;

3. Algoritmos de simulação quântica.

Os algoritmos baseados na versão quântica da Transformada de Fourier estão asso- 
ciados a obtenção de formas estritamente mais eficientes que as alternativas clássicas de reversão de algumas das principais funções de mão-única conhecidas, como a fatoração de inteiros e a solução do logaritmo discreto.

Os algoritmos de busca, tendo como o principal representante o algoritmo de busca de Grover, utilizam a propriedade de paralelismo quântico na obtenção de métodos de busca de informação em bases de dados não organizadas de forma mais eficiente que os algoritmos clássicos conhecidos.

Os algoritmos de simulação quântica estão associados a utilização de circuitos quânticos na simulação de sistemas físicos quânticos, tarefa bastante dispendiosa para os computadores clássicos. Estes algoritmos são bastante eficientes nessa tarefa, sendo um indício da contribuição que a computação quântica pode dar a tarefa de modelagem molecular, dentre outras, tão importante em diversas indústrias fundamentais como as de semicondutores e fármacos por exemplo.

Considerando que os métodos criptográficos atuais, tal como discutido no capítulo 2, utilizam como fundamento de segurança a incapacidade da computação clássica em tratar algumas classes de problemas matemáticos de forma eficiente, alguns dos algoritmos quânticos anteriores representam então uma ameaça real a segurança desses métodos criptográficos. Na próxima seção será discutido o impacto que a eficiência dos algoritmos quânticos pode ter sobre as técnicas de criptologia clássicas, sendo mostrado como o algoritmo de Shor, principal resultado da computação quântica, pode ser utilizado para resolver o problema da fatoração de inteiros de forma bastante eficiente.

\subsection{Impacto da computação quântica sobre a criptolo- gia clássica}

Como apresentado na seção 2.2, existem protocolos de criptografia simétrica que têm sua segurança provada de um ponto de vista da teoria da informação, como é o caso da cifra de Vernam, com segurança demonstrada por Shannon. Nesse caso a computação quântica por mais eficiente que seja não representa nenhuma ameaça a segurança. Contudo, as dificuldades da utilização dos algoritmos simétricos como a Cifra de Vernam levaram ao desenvolvimento dos protocolos simétricos com chaves de tamanhos 
definidos e dos protocolos assimétricos, cujo núcleo estão as funções definidas anteriormente como funções de mão-única. Estes algoritmos estão fortemente relacionados a dificuldade computacional de se inverter tais funções. Neste ponto a eficiência da computação quântica se torna bastante relevante.

Na seção 3.1 foram apresentadas algumas propriedades dos algoritmos quânticos que indicam a eficiência destes diante de seus análogos clássicos conhecidos. Nesta seção será mostrado como os algoritmos quânticos podem ser utilizados para resolver problemas práticos de processamento. Como o algoritmo RSA é o algoritmo criptográfico assimétrico mais amplamente utilizado, sendo também a principal fonte de chaves de sessão para os protocolos simétricos de cifra de bloco, uma técnica que demonstre ser capaz de quebrá-lo tem grande importância, com impactos desde a Internet a diversos tipos de sistemas de comunicação.

A função de mão-única que está no núcleo do algoritmo RSA consiste no problema da fatoração de inteiros. Esse, contudo, é um dos problemas em que a computação quântica é conhecidamente mais eficiente que a computação clássica. Na seção seguinte será feita uma introdução de como a teoria sobre circuitos quânticos, apresentada anteriormente, pode ser utilizada para construir um algoritmo quântico capaz de quebrar o algoritmo RSA, ou seja, reverter a função de mão-única, em tempo viável.

\subsubsection{Transformada de Fourier Quântica}

Assim como a Transformada de Fourier clássica a versão quântica é uma ferramenta extremamente versátil na solução de um conjunto diverso de problemas, sendo que uma teoria generalizada desta é responsável pela obtenção da transformação utilizada por muitos dos algoritmos quânticos mais interessantes.

Dois dos algoritmos quânticos mais importantes, o algoritmo de fatoração de Shor e o logaritmo discreto, são baseados na transformada de Fourier. A ação da versão quântica da transformada de Fourier sobre uma base ortonormal $|0\rangle, \ldots,|N-1\rangle$ é definida por um operador linear que age sobre os estados da forma:

$$
|j\rangle \rightarrow \frac{1}{\sqrt{N}} \sum_{k=0}^{N-1} e^{2 \pi i j k / N}|k\rangle .
$$


A ação sobre um estado arbitrário é definida então por:

$$
\sum_{j=0}^{N-1} x_{j}|j\rangle \rightarrow \sum_{j=0}^{N-1} y_{k}|k\rangle,
$$

com cada amplitude $y_{k}$ sendo a transformada de Fourier discreta das amplitudes $x_{j}$.

A transformada de Fourier Quântica, atribuição dada para a versão definida pela equação (3.34), é uma transformação unitária como pode ser verificado. Isso resulta que a versão quântica da transformada de Fourier pode ser implementada por um circuito quântico por meio da ação de portas quânticas. Uma forma de se obter o circuito quântico que implementa a transformada Quântica de Fourier pode ser obtida considerando $N=2^{n}$, com $n$ inteiro e a base computacional de n-qubits $|0\rangle, \ldots,\left|2^{n}\right\rangle$, e utilizando a representação binária $j=j_{1} j_{2} \ldots j_{n}$, i.e., $j=j_{1} 2^{2 n-1}+j_{2} 2^{2 n-2}+\ldots+j_{n} 2^{0}$ e a notação $0 . j_{l} j_{l+1} \ldots j_{m}$ para a fração binária $j_{l} / 2+j_{l+1} / 4+\ldots j_{m} / 2^{2 m-l+1}$. Assim, obtém-se a forma útil de representação da transformada dada por:

$$
\left|j_{1} \ldots j_{n}\right\rangle \rightarrow \frac{\left(|0\rangle+e^{2 \pi i 0 . j_{n}}|1\rangle\right)\left(|0\rangle+e^{2 \pi i 0 . j_{n-1} j_{n}}|1\rangle\right) \ldots\left(|0\rangle+e^{2 \pi i 0 . j_{1} j_{2} \ldots j_{n}}|1\rangle\right)}{2^{n / 2}}
$$

A equação (3.36) permite obter o circuito quântico para a transformada de Fourier mostrado na figura 12, que possibilita uma prova direta do fato da transformada quântica de Fourier ser unitária além de ajudar na compreensão de outros algoritmos baseados nessa trasformada como será visto na seção 3.2.2. O circuito apresentado na figura 12 utiliza a definição:

$$
R_{k} \equiv\left[\begin{array}{cc}
1 & 0 \\
0 & e^{2 \pi i / 2^{k}}
\end{array}\right]
$$

Classicamente a rotina FFT (Fast Fourier Transform) necessita de aproximadamente $N \log (N)=n 2^{n}$ operações para realizar a transformada de $2^{n}$ números. A transformada de Fourier quântica pode ser feita em um número de passos da ordem de $\log ^{2}(N)=n^{2}$. Isso representa um ganho de desempenho exponencial.

O resultado da utilização da transformada de Fourier quântica indica que um computador quântico poderia ser utilizado para calcular a Transformada de Fourier de um 


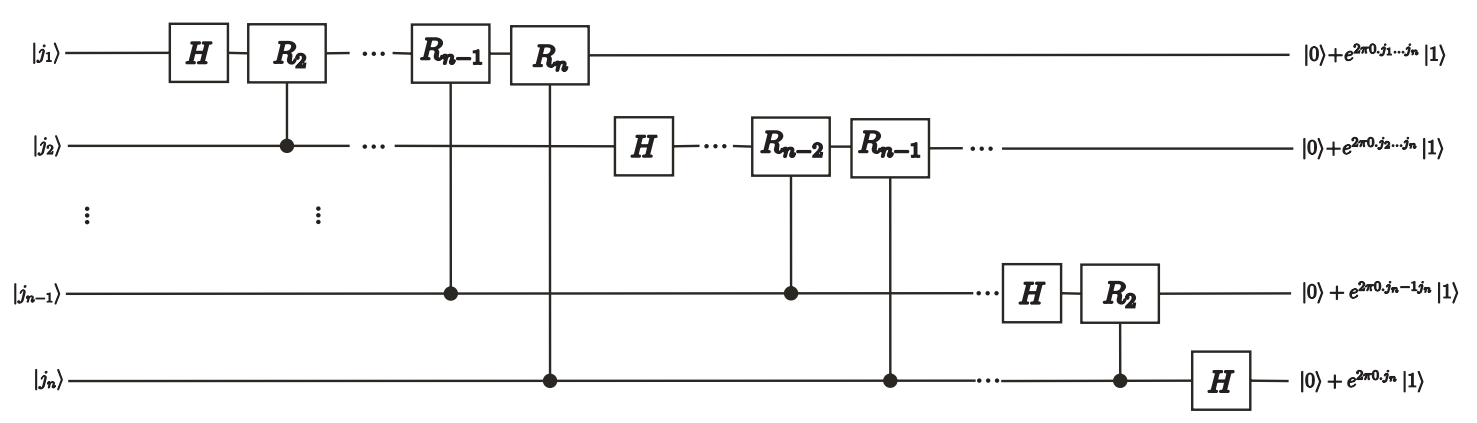

Fig. 12: Circuito quântico para Transformada Quântica de Fourier

vetor $2^{n}$ números complexos muito rapidamente, sendo isso extremamente útil em um vasto campo de aplicações. Contudo, a transformada está sendo realizada nas amplitudes dos estados quânticos, a informação oculta, sendo que nesse caso ao ocorrer a medida do estado de saída, o qubit colapsará em $|0\rangle$ ou $|1\rangle$. Isso faz com que seja possível calcular $2^{n}$ de forma muito eficiente, entretanto este resultado não é acessível diretamente. Esse problema atinge o núcleo da dificuldade do projeto dos algoritmos quânticos. Um exemplo de como superar essa dificuldade é representado pelo algoritmo de Shor, que utiliza a Transformada de Fourier Quântica por meio do procedimento conhecido como estimativa de fase e uma aplicação conhecida como busca de ordem para implementar a solução da fatoração de inteiros utilizando uma medida indireta. O algoritmo é capaz de se beneficiar da informação ocultada nas amplitudes dos estados quânticos e realizar a tarefa em tempo polinomial com o tamanho do número inteiro. Esse resultado é considerado um dos mais importantes obtidos no campo da computação quântica e informação quântica devido ao grande impacto em segurança da informação e a forte indicação que aponta da capacidade de manipulação de informação por computadores quânticos

\subsubsection{Procedimento de Estimativa de Fase}

A transformada quântica de Fourier mostra sua verdadeira utilidade quando utilizada em conjunto por outros algoritmos quânticos. Isso pode ser visto no importante procedimento conhecido como Estimativa de Fase, que fundamenta diversos dos mais importantes resultados téoricos da computação quântica. O procedimento pode ser entendido por meio da tarefa geral em que dado um operador unitário $U$ com autovetor $|u\rangle$ e autovalor $2^{2 \pi i \varphi}$, com $\varphi$ desconhecido, estimar $\varphi$ (ver apêndice B para descri- 
ção detalhada dos aspectos algébricos relacionados a autovetores e autovalores). Neste caso considera-se a existência de elementos abstratos, referenciados na literatura como caixas-pretas ou oráculos, capazes de preparar o estado $|u\rangle$ e realizar a operação $U^{2^{j}}$ controlada para alguns inteiros não-negativos $j$. A utilização do conceito de caixaspretas está relacionada ao fato de que o procedimento de estimativa de fase é na verdade uma sub-rotina, sendo que esta pode ser combinada com outras sub-rotinas responsáveis pelas tarefas relacionada às caixas-pretas. Algumas combinações para a sub-rotina de estimativa de fase permitem resolver problemas computacionais relevantes, o que será visto com maiores detalhes na seção seguinte.

A operação do procedimento de estimativa de fase utiliza dois registros: o primeiro de t-qubits no estado $|0\rangle$ e o segundo com quantos qubits forem necessários para armazenar $|u\rangle$. O circuito que implementa a estimativa de fase é iniciado por uma aplição de Hadamard sobre o primeiro registro seguida da aplicação da transformação unitária $U$-controlada elevada a sucessivas potências de 2 . O estado do primeiro registro é dado então por:

$$
\begin{array}{r}
\frac{1}{2^{t / 2}}\left(|0\rangle+e^{2 \pi i 2^{t-1} \varphi}|1\rangle\right)\left(|0\rangle+e^{2 \pi i 2^{t-2} \varphi}|1\rangle\right) \ldots\left(|0\rangle+e^{2 \pi i 2^{0} \varphi}|1\rangle\right)= \\
=\frac{1}{2^{t / 2}} \sum_{k=0}^{2^{t}-1} e^{2 \pi i \varphi k}|k\rangle .
\end{array}
$$

Com $\varphi$ podendo ser expresso com exatamente $t$ bits, pode-se então escrever $\varphi=$ $0 . \varphi_{1} \ldots \varphi_{t}$. Isso faz com que a equação (3.38) possa ser reescrita da forma:

$$
\begin{aligned}
\frac{1}{2^{t / 2}}\left(|0\rangle+e^{2 \pi i 0 . \varphi_{t}}|1\rangle\right)\left(|0\rangle+e^{2 \pi i 0 . \varphi_{t-1} \varphi_{t}}|1\rangle\right) \ldots(|0\rangle & \left.+e^{2 \pi i 0 . \varphi_{1} \varphi_{2} \ldots \varphi_{t}}|1\rangle\right)= \\
& =\frac{1}{2^{t / 2}} \sum_{k=0}^{2^{t}-1} e^{2 \pi i \varphi k}|k\rangle .
\end{aligned}
$$

O segundo estágio do circuito é representado pela aplicação da transformada de Fourier quântica inversa. Da equação (3.39) tem-se que esta aplicação fornece exatamente o estado-produto $\left|\varphi_{1} \ldots \varphi_{t}\right\rangle$, fazendo com que uma medida na base computacional leve então a $\varphi$, com precisão proporcional a $t$. Um diagrama resumido do circuito 
responsável pela implementação do procedimento de estimativa de fase é mostrado na figura 13 e um resumo dos passos da realização do procedimento.

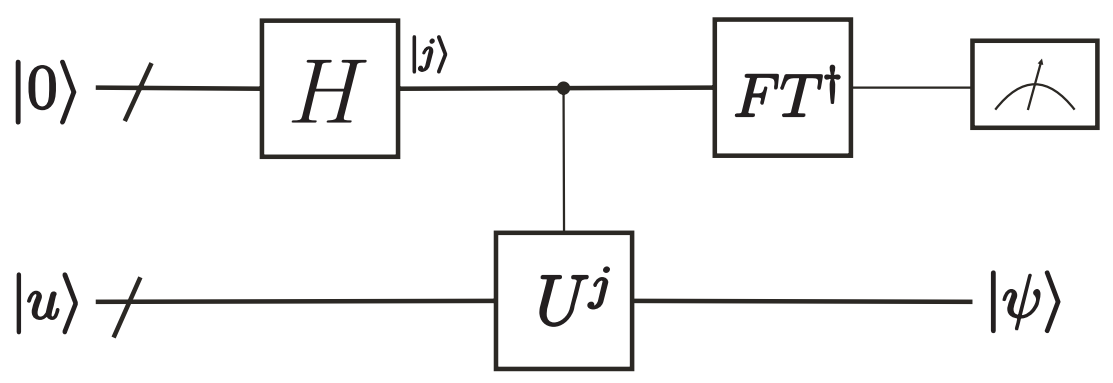

Fig. 13: Circuito quântico para Estimativa de Fase

\subsubsection{Problema da busca de ordem}

O procedimento de estimativa de fase descrito anteriormente pode ser utilizado na resolução de uma grande variedade de problemas relevantes. Uma das mais importantes aplicações do procedimento de estimativa de fase é representada pela solução do problema da busca de ordem. A solução eficiente deste problema tem grande impacto na operação dos principais protocolos de criptografia assimétrica descritos no capítulo anterior, como será visto na seção seguinte.

Para inteiros positivos $x$ e $N, x<N$, sem fatores comuns, a busca de ordem de $x$ módulo $N$ é definida como o menor inteiro positivo $r$ tal que $x^{r} \bmod N=1$. O problema da busca de ordem consistem em se determinar a ordem, sendo $x$ e $N$ conhecidos (NIELSEN; CHUANG, 2000).

Um algoritmo quântico de busca de ordem pode ser derivado diretamente do procedimento de estimativa de fase descrito anteriormente por meio da aplicação deste procedimento ao operador unitário $U$, tal que

$$
U|y\rangle \equiv|x y(\bmod N)\rangle,
$$

com $y \in\{0,1\}^{L}$. Neste caso, quando $N \leq y \leq 2^{L}-1$ é usada a convenção de que $x y($ mod $N$ ) é novamente $y$. Assim, $U$ só atua de forma não-trivial para $0 \leq y \leq N-1$. Isso faz com que os estados definidos por 


$$
\left|u_{s}\right\rangle \equiv \frac{1}{\sqrt{r}} \sum_{k=o}^{r-1} \exp \left[\frac{-2 \pi i s k}{r}\right]\left|x^{k} \bmod N\right\rangle,
$$

para inteiros $0 \leq s \leq r-1$ são auto-estados de $U$, uma vez que:

$$
\begin{aligned}
U\left|u_{s}\right\rangle & =\frac{1}{\sqrt{r}} \sum_{k=o}^{r-1} \exp \left[\frac{-2 \pi i s k}{r}\right]\left|x^{k+1} \bmod N\right\rangle \\
& =\exp \left[\frac{2 \pi i s k}{r}\right]\left|u_{s}\right\rangle .
\end{aligned}
$$

A partir da equação (3.42) e do procedimento de estimativa de fase pode-se obter, com alta precisão, os valores correspondentes $\exp (2 \pi i s / r) \mathrm{e}$, portanto, a ordem $r$.

Contudo, existe duas condições necessárias para a utilização do procedimento de estimativa de fase, relacionadas a obtenção das caixas-pretas utilizadas no procedimento: deve ser possível implementar uma operação $U^{2^{j}}$-controlada, para qualquer inteiro $j$, e deve ser possível preparar um auto-estado $\left|u_{s}\right\rangle$ com autovalor não-trivial, ou ao menos uma superposição de tais auto-estados. A primeira condição é satisfeita usando-se o procedimento chamado de exponenciação modular, com o qual é possível implementar a sequência inteira de operações $U^{2^{j}}$-controlada, necessária para a estimativa de fase (BRYLINSKI; CHEN, 2002).

A segunda condição exige a preparação de $\left|u_{s}\right\rangle$ sem o conhecimento de $r$. Uma propriedade que permite tal operação consiste no fato que

$$
\frac{1}{\sqrt{r}} \sum_{s=0}^{r-1}\left|u_{s}\right\rangle=|1\rangle
$$

Assim, a estimativa de fase pode ser feita por meio da utilização de $t=2 L+1+$ $[\log (2+1 / 2 \epsilon)]$ qubits no primeiro registro e da utilização de do estado $|1\rangle$ no segundo registro. Assim, para cada $s$ no intervalo de 0 até $r-1$ obtém-se a estimativa de fase $\varphi \approx r / s$ com precisão de $2 L+1$ bits e com probabilidade de ao menos $(1-\epsilon) / r$.

A solução do problema se completa com a obtenção de $r$ a partir do resultado obtido para a estimativa de fase $\varphi \approx r / s$. Isso pode ser feito por meio do algoritmo de frações contínuas (BOUWMEESTER, 2000) que permite, dado $\varphi$, obter de forma eficiente 
os números $s$ e $r$.

Dessa forma, foi mostrado de forma resumida que a partir da transformada quântica de Fourier é possível obter um procedimento de estimativa de fase que permitiu a construção de um algoritmo eficiente para a busca de ordem. Esse é um dos principais resultados da computação quântica até o momento, especialmente pelos desdobramentos que este causa na área de segurança da informação. Na seção seguinte é mostrado como o algoritmo de busca de ordem pode ser utilizado na obtenção de um algoritmo útil para resolver um problema computacional prático.

\subsubsection{Como quebrar o RSA com o algoritmo de Shor}

O procedimentos de estimativa de fase e o algoritmo de busca de ordem têm sua utilidade demonstrada na solução proposta por Peter Shor (SHOR, 1997) para o problema da fatoração, solução que permite quebrar o RSA, i.e., resolver o problema da fatoração, de forma muito mais eficiente que qualquer solução clássica conhecida. $\mathrm{O}$ algoritmo de Shor consiste na redução do problema da fatoração ao problema da busca de ordem e na aplicação do procedimento descrito na seção anterior. Este pode ser utilizado para decifrar a mensagem codificada por meio do algoritmo RSA, $E=\left(P^{a}\right) \bmod N$, por meio dos passos resumidos a seguir. Primeiramente, utilizando novamente os atores tradicionais da criptologia apresentados no capítulo 2, Eva obtém a chave pública de Bob $K_{B}=(\mathrm{a}, \mathrm{N})$ de um banco de dados públicos e executa o processo:

1. Primeiro, ela calcula a ordem de $E$ no sentido módulo $N=1$ utilizando o procedimento descrito na seção 3.2.3 e denota o valor encontrado por $r$ que é $\left(\left(P^{a}\right)^{r}\right)$ $\bmod N=1$. Esse passo requer que $E$ e $N$ sejam primos relativos. Caso isso não aconteça, Eva pode aplicar o algoritmo de Euclides (seção A.2) para eliminar fatores comuns, o que provê $p$ e $q$.

2. Em seguida Eva calcula o módulo $r$ multiplicativo inverso de $a$. A existência do inverso $b^{\natural}$ requer que $a$ seja co-primo de $r$. Desde que $\left(E^{r}\right) \bmod N=1$ e do teorema de Euler sabemos que $\left(E^{\varphi(N)}\right) \bmod N=1$, portanto $\varphi(N)=k \cdot r$ para um certo inteiro $k$, que é fator primo de $r$ formando um subconjunto de $\varphi(N)$. Da relação $m d c(\varphi(N), a)=1$, sabemos que $a$ e $\varphi(N)$ são primos relativos, e por causa 
do algoritmo RSA sabemos que $a$ é co-primo de $r$ também.

3. Eva executa o algoritmo RSA novamente de forma que $(a \cdot b) \bmod \varphi(N)=1$, enquanto no passo 2 era $\left(a \cdot b^{\natural}\right) \bmod r=1$ e $\varphi(N)=k \cdot r$, portanto $b^{\natural}=b+k \cdot r$.

4. De posse de $b^{\natural}$ Eva utiliza este no processo de decifragem no lugar do fator $b$ desconhecido, obtendo:

$$
\left(\left(P^{a}\right)^{b^{\natural}}\right) \bmod N=\left(P^{a b+a k r}\right) \bmod N=\left(P^{a b} \cdot\left(P^{a r}\right) k\right) \quad \bmod N=P,
$$

onde Eva obtém que o primeiro termo módulo $N$ é igual a $P$ enquanto, devido a definição de $r$, o segundo termo se torna 1. Assim, Eva consegue obter a mensagem original sem qualquer conhecimento a cerca de $b$, i.e., ela utilizou a conexão especial existente entre $b$ e $b^{\natural}$ para quebrar o algoritmo RSA.

Com o intuito de comparar as diversas formas possíveis de se atacar o algoritmo RSA e o tempo necessário para alcançar esse objetivo, é considerado o seguinte cenário: como referência se considera um computador clássico e um computador quântico capazes de realizar $10^{12}$ operações por segundo. Varia-se $n=\log _{10}(N)$ (o tamanho de $N$ ) de 16 bits a 1024 bits para os métodos de ataque conhecidos, o que é mostrado a seguir. Na comparação é utilizada a notação tradicional de complexidade computacional, a notação de Lamport $(O(\cdot))$, que descreve como o tamanho das entradas influi no tempo de execução de um algoritmo, descrevendo os limites assintóticos superiores para a magnitude de uma função. Os métodos de comparação são, com respectiva complexidade computacional:

(i) BF: método de força bruta clássico de procura por inteiros de 2 a $\lceil\sqrt{N}\rceil$ com complexidade $O(\sqrt{N})$;

(ii) BC: melhor método clássico conhecido, requerendo o número de operações

$$
O\left(\exp \left[c \cdot \log _{10}^{1 / 3}(N) \log _{10}{ }^{2 / 3}\left(\log _{10}(\mathrm{~N})\right)\right]\right)
$$

(iii) S: fatoração de Shor com $O\left(\log _{10}(N)^{3}\right)$. 
A figura 14 apresenta as três curvas dos diferentes métodos para o número de operações $\sigma$ requisitado para quebrar uma chave RSA de tamanho $N$. A figura 15 mostra o tempo $s$ em segundos, considerando o desempenho de $10^{12}$ operações por segundo, requisitado pelos diferentes métodos para quebrar uma chave RSA de tamanho $N$. Fica evidente visualmente a grande eficiência do algoritmo de Shor quando comparado aos algoritmos clássicos. A tabela 3 resume alguns pontos principais que decorrem das curvas apresentadas.

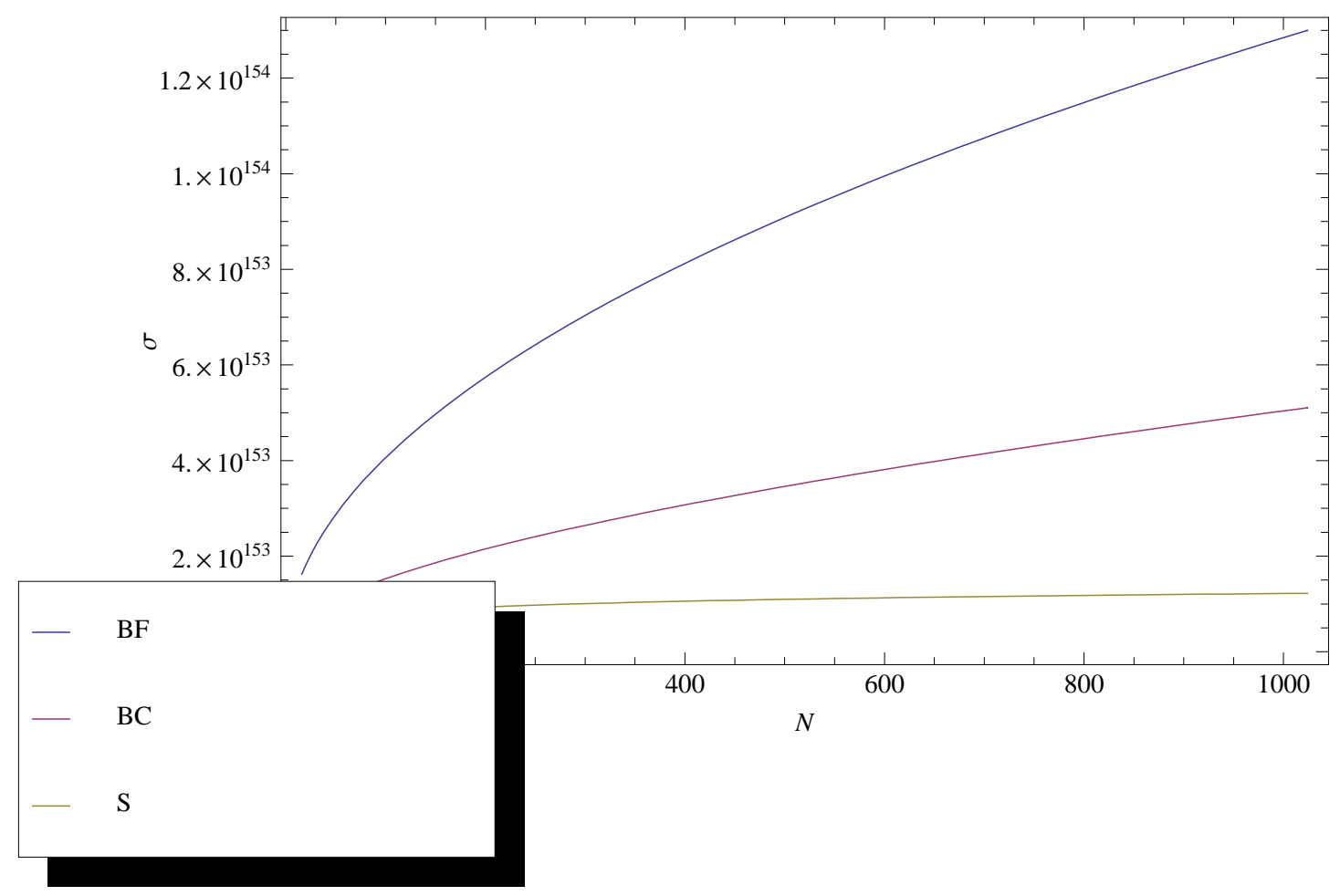

Fig. 14: Número de operações $\sigma$ requisitado para quebrar uma chave RSA de tamanho $N$ utilizando diferentes métodos

Tab. 3: Métodos utilizados para quebrar o RSA e complexidade requerida

\begin{tabular}{ccccc}
\hline método & $\begin{array}{c}n=128 \\
(\mathrm{~s})\end{array}$ & $\begin{array}{c}n=128 \\
(\text { anos })\end{array}$ & $\begin{array}{c}n=1024 \\
(\mathrm{~s})\end{array}$ & $\begin{array}{c}n=1024 \\
(\text { anos })\end{array}$ \\
\hline $\mathrm{BF}$ & $1,8 \cdot 10^{7}$ & 0,58 & $1,3 \cdot 10^{142}$ & $4 \cdot 10^{134}$ \\
$\mathrm{BC}$ & $6 \cdot 10^{-4}$ & $1,9 \cdot 10^{-11}$ & $3,5 \cdot 10^{8}$ & 11,29 \\
$\mathrm{~S}$ & $2 \cdot 10^{-3}$ & $6,6 \cdot 10^{-14}$ & $\mathbf{0 , 0 1}$ & $3,4 \cdot 10^{-11}$ \\
\hline
\end{tabular}

A tabela 3 evidencia que a computação quântica, por meio da implementação do algoritmo de fatoração de Shor, pode tratar o problema da fatoração de inteiros de forma estritamente mais eficiente que os algoritmos clássicos disponíveis. O algoritmo de Shor representa um ganho de eficiência exponencial comparado ao melhor algoritmo clássico 


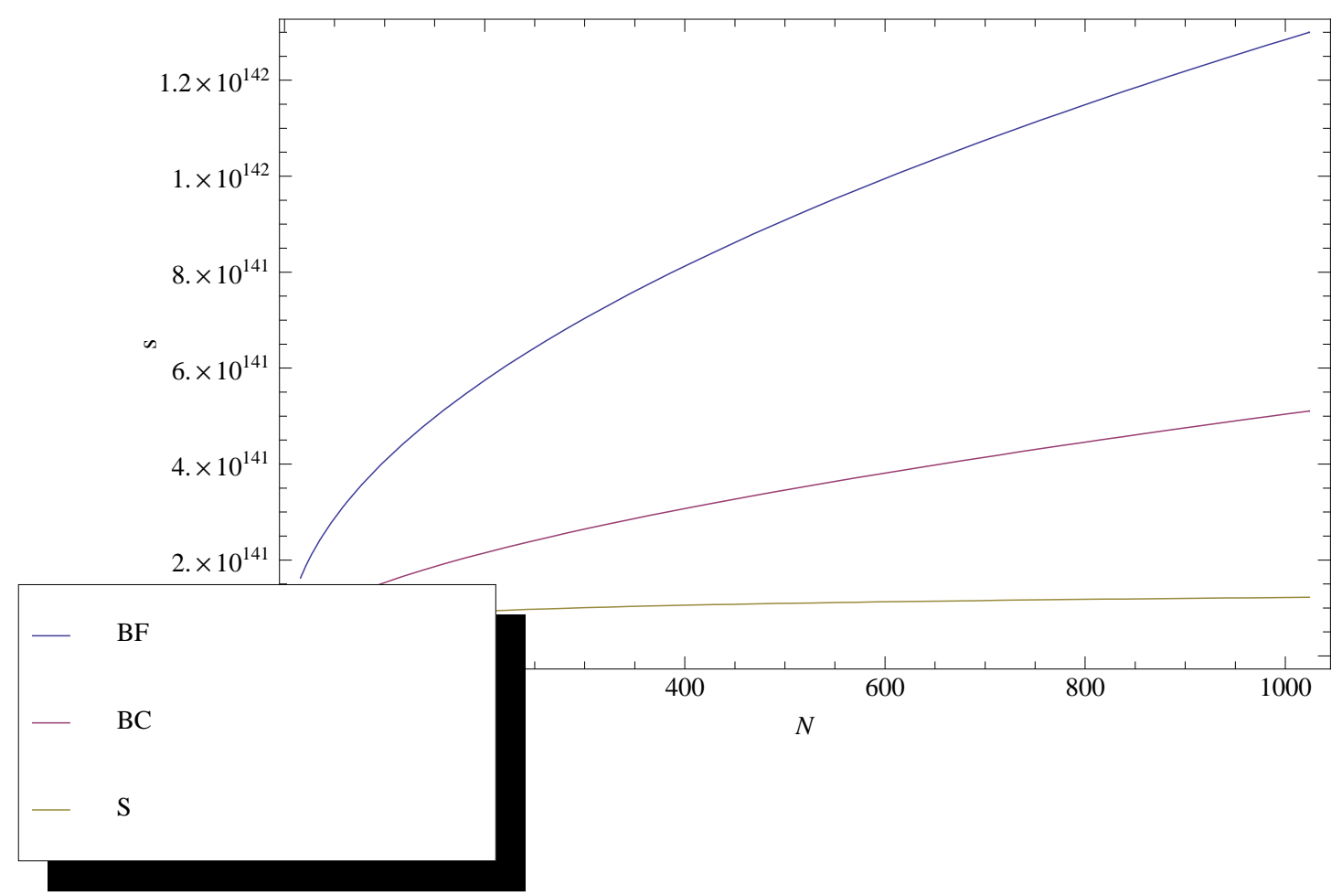

Fig. 15: Tempo $s$ em segundos requisitado para quebrar uma chave RSA de tamanho $N$ utilizando diferentes métodos

conhecido, como pode ser visto graficamente nas figuras 14 e 15 . Estes resultados indicam que uma implementação física do algoritmo de Shor com qubits suficientes para tratar uma chave de, por exemplo, 1024 bits, tamanho recorrente verificado em execuções práticas do algoritmo, tornaria o algoritmo RSA impraticável. A implementação desse algoritmo quântico permitira obter a chave de 1024 bits utilizada em apenas 0, 01 segundos, fração de tempo irrisória compara aos $3,5 \cdot 10^{8}$ segundos exigidos pelo melhor método clássico, tempo este que têm sido utilizado como referência para se medir a segurança de uma chave RSA. Um aspecto relevante na implementação do algoritmo de Shor consiste no número de qubits necessários para a obtenção de uma chave RSA de $n$ bits. A precisão exigida em casos práticos faz necessária a utilização de no mínimo $\approx 1,6 n$ e no caso ideal $\approx 2 n$ qubits para obter uma chave RSA de $n$ bits (MENEZES; OORSCHOT; VANSTONE, 2001; NIELSEN; CHUANG, 2000).

Adicionalmente, o algoritmo de estimativa de fase apresentado na seção 3.2.2 pode ser utilizado de forma semelhante ao algoritmo de Shor na obtenção de um algoritmo quântico capaz de reverter de forma tão eficiente quanto aquela verificada no caso da fatoração de inteiro o problema dos logarítmos discretos, fundamento utilizado em di- 
versos outros métodos criptográficos assimétricos, tal como o algoritmo de ElGamal apresentado na seção 2.4.1.

\subsection{Considerações finais do capítulo}

Os resultados teóricos apresentados anteriormente levam a uma grande preocupação quanto a segurança dos métodos criptográficos amplamente utilizados hoje diante da presença da computação quântica. Há contudo dois fatores que devem ser considerados neste aspecto.

O primeiro aspecto refere-se ao fato de que apesar dos bons resultados teóricos obtidos pela computação quântica para alguns problemas computacionais, ainda não se sabe com exatidão quais classes de problemas matemáticos os computadores quânticos são mais eficientes que os clássicos. A existência de um problema matemático de tratamento difícil mesmo para computação quântica poderia ser uma alternativa para a obtenção de um método criptográfico seguro mesmo na presença da computação quântica.

O segundo aspecto refere-se ao fato de que o risco potencial representado pela computação quântica somente se tornaria efetivo diante da capacidade de se implementar os conceitos teóricos relacionados a computação quântica em sistemas físicos com um número mínimo e estável de qubits suficiente para cálculos práticos. Neste aspecto, a despeito do grande avanço realizado na teoria de computação quântica, há ainda diversos desafios a serem superados na obtenção de uma implementação de computação quântica escalável e útil para fins práticos.

Diversas abordagens de implementação física para computação quântica têm sido propostas nos últimos anos, sendo este um tópico de pesquisa que tem movimentado diversos dos mais importantes institutos de pesquisa do mundo. Não cabe aqui uma exposição das técnicas disponíveis e utilizadas na obtenção de computação quântica experimental, mesmo porque este é um vasto campo do conhecimento tendo requisitos teóricos específicos. Contudo, é importante citar ao menos duas implementações físicas que são relevantes quando se considera o futuro dos métodos criptográficos atuais.

A primeira delas consiste na demonstração da utilização da técnica de Ressonância Magnética Nuclear (RMN) na implementação de computação quântica realizada pelo 
grupo de pesquisadores do IBM Almaden Research Center, um dos centro de pesquisa e desenvolvimento da IBM Corporation, em dezembro de 2001 (NIELSEN; CHUANG, 2000). Sob a direção de Isaac L. Chuang, o grupo implementou 7 qubits por RMN, sendo estes utilizados na execução do algoritmo de fatoração de Shor, executando eficientemente a fatoração do número 15 em seus fatores primos 3 e 5 . Apesar da simplicidade da tarefa e da dificuldade em tornar a técnica escalável, o experimento tem como importante resultado a demonstração da validade das previsões teóricas da computação quântica, como o cálculo por meios físicos da transformada de Fourier quântica. O experimento mostrou que é possível levar a computação quântica a uma realidade de engenharia.

A segunda implementação é mais recente, tendo sido anunciada inicialmente em fevereiro de 2007 pela D-Wave Systems, quando foi demonstrada a operação do processador quântico Orion de 16 qubits, uma implementação estável, escalável e integrável a computação clássica atual (MOORE, 2007). O anúncio inicial foi sucedido em meses posteriores por novos anúncio de melhorias e aumento de capacidade, como o feito em novembro de 2007, onde foi demonstrada a operação na Supercomputing 2007 Conference de um processador quântico de 28 qubits executando tarefas básicas de processamento de informação. A empresa divulgou publicamente um cronograma de atividades, com o anúncio programado de um processador quântico de 512 qubits no segundo quadrimestre de 2008 e de um processador quântico de 1024 qubits até o fim de 2008. Estão nos planos anunciados pela D-Wave ainda a disponibilização do processador quântico Orion comercialmente por meio de um serviço on-line até o fim de 2009, sendo o serviço voltado para o tratamento de problemas de reconhecimento de padrões, problemas de busca de dados, otimização, análise de risco financeira e simulações físicas para ciências químicas e de materiais (D-WAVE, 2008).

Apesar da grande atenção e recursos atraídos pela D-Wave após o anúncio do Orion em 2007, há ainda muitas dúvidas apontadas por especialistas em computação quântica relacionadas ao processador quântico desenvolvido (NEWS@NATURE, 2007; MINKEL, 2007). Em especial tem-se o fato de que a técnica utilizada não foi formalmente publicada em periódicos científicos ou documentada numa solicitação de registro de patente. A D-Wave apresenta como argumentos o caráter estratégico da tecnologia desenvolvida e a necessidade de proteção da propriedade intelectual relacionada a esta. 
Independentemente da polêmica associada a obtenção comercial de computadores quânticos, os anúncios de 2007 são um forte indício de que se os sistemas atuais de criptografia não estão em risco no momento atual, poderão ser totalmente invalidados em um período próximo. Essa conclusão levou diversos pesquisadores a procurar soluções alternativas para construção de métodos criptográficos. O capítulo seguinte apresenta algumas alternativas disponíveis, mostrando que uma das mais promissoras destas é representada pela obtenção de um método criptográfico baseado em um sistema quântico. Neste contexto serão introduzidas algumas contribuições deste trabalho na obtenção de um método de segurança baseado em sistemas quânticos voltado para ambientes práti$\cos$. 


\section{Criptografia quântica}

Os resultados teóricos relacionados à computação quântica apresentados no capítulo anterior mostram que os sistemas criptográficos atuais podem se tornar vulneráveis na presença de uma implementação bem sucedida de computação quântica. O sucesso de algumas realizações experimentais de computação quântica anunciadas nos últimos anos e a perspectiva de escalonamento de algumas técnicas de implementação indicam que a potencial vulnerabilidade dos sistemas criptográficos atuais pode se efetivar em um período próximo. Estes são fatores suficientes para a busca de uma alternativa para a obtenção de novos métodos criptográficos.

Tem-se verificado duas abordagens distintas na tentativa de se obter um sistema criptográfico seguro mesmo na presença da computação quântica. A primeira desta consiste na utilização, semelhante ao que acontece no caso dos protocolos assimétricos convencionais, de problemas matemáticos de difícil tratamento computacional. O que se convencionou chamar de criptográfica pós-quântica é o conjunto de técnicas criptográficas fundamentadas em problemas para os quais não se conhece uma solução eficiente mesmo em computadores quânticos. Alguns dos conceitos utilizados na construção destes algoritmos são os grupos não-abelianos, o problema da decodificação de síndrome, redução de reticulados (lattice reduction), sistemas quadráticos multivariados, dentre outros (REGEV, 2002).

Os algoritmos pós-quânticos têm como grande benefício a manutenção da atual infra-estrutura de segurança para telecomunicações, já que tem mecanismo de funcionamento semelhante aqueles já utilizados. Contudo, uma importante questão envolvendo os algoritmos criptográficos pós-quânticos é representada pelo fato de que os fundamentos utilizados como premissa de segurança desses métodos não tem uma demonstração formal, i.e., não foi provado, para nenhum dos problemas utilizados nos algoritmos 
pós-quânticos propostos até o momento, que é impossível desenvolver um algoritmo clássico ou quântico capaz de tratar de forma eficiente o problema matemático utilizado como premissa de segurança.

Há uma segunda abordagem na obtenção de um sistema criptográfico que se mantenha seguro mesmo na presença de computação quântica, sendo uma abordagem completamente distinta daquela utilizada pelos métodos atuais. Algumas das propriedades de sistemas quânticos apresentadas anteriormente podem ser utilizadas na obtenção de novos sistemas criptográficos que se mantém seguros mesmo na presença de computação quântica. O conjunto de técnicas criptográficas que fazem uso das propriedades de sistemas quânticos como mecanismo de segurança é conhecido como criptografia quântica.

Na seção 4.1 são apresentados os principais conceitos relacionados a criptografia quântica e os motivos que tornam essa uma interessante alternativa para os métodos atuais, apresentados os principais algoritmos criptográficos quânticos conhecidos, o comportamento de um protocolo de criptografia quântica em um cenário típico de comunicação e o desenvolvimento de uma abordagem de criptografia quântica voltada para aplicações práticas de telecomunicações. A seção 4.2 mostra como a abordagem desenvolvida pode ser integrada a redes de telecomunicações nos moldes daquelas existentes, apresenta as técnicas de implementação de protocolos de criptografia quântica disponíveis e discute os possíveis cenários de utilização de métodos de criptografia quântica e as principais dificuldades encontradas nessa nova abordagem.

\subsection{Criptografia quântica}

Na seção 2.2 foi mostrado que somente a Cifra de Vernam consiste em uma técnica de criptografia clássica com segurança demonstrada formalmente. Contudo, o problema de se estabelecer uma longa chave criptográfica, requisito fundamental para a segurança do método, a ser utilizada em um cenário prático se mostrou pouco viável, levando a formulação dos algoritmos de criptografia assimétrica como mecanismo de estabelecimento de chaves de tamanhos definidos para a utilização conjunta com métodos criptográficos simétricos. Apesar das vulnerabilidades potenciais representadas 
pela computação quântica aos métodos criptográficos assimétricos, a Cifra de Vernam é resistente mesmo na presença de computação quântica. Com isso, um método que permitisse o estabelecimento de longas chaves criptográficas de forma segura tornaria possível a utilização da Cifra de Vernam em casos práticos.

As propriedades verificadas em sistemas quânticos podem ser utilizadas, semelhante a utilização destas no processamento de informação, na obtenção de um novo método criptográfico baseado em propriedades do meio físico utilizado na transmissão de informação. A utilização de sistemas quânticos na transmissão de dados, especialmente com a utilização de fótons manipulados individualmente (ver B.4 para uma descrição detalhada sobre o comportamento quântico da luz), apresenta algumas características não verificadas em sistemas clássicos.

As principais características que fazem com que a utilização de sistemas quânticos na transmissão de informação seja vantajosa são principalmente a existência da limitação física para medição simultânea de certas grandezas, propriedade conhecida como princípio da incerteza de Heisenberg (ver seção B.5), fazendo com que sinais quânticos, sejam invariavelmente alterados quando ocorre qualquer tipo de interferência. A impossibilidade de não se alterar um sinal quântico dada uma interferência leva a um teorema que é utilizado como um dos fundamentos na obtenção de um método de transmissão seguro baseado em propriedades quânticas. O teorema da não-clonagem estabelece que é impossível copiar um sinal quântico sem alterar de forma significativa o sinal original (GOBBY; YUAN; SHIELDS, 2005) (ver seção B.6 para uma descrição detalhada e uma demonstração desse teorema). Essas duas propriedades formam os fundamentos para o desenvolvimento de um novo método de distribuição de chaves criptográficas.

Uma grande motivação para a utilização de fenômenos quânticos na transmissão de informação reside no fato do maior desenvolvimento das técnicas de construção de dispositivos quânticos para transmitir informação do que aqueles necessários para armazenar ou processar informação quântica. Esse fato permitiu a obtenção de alguns dispositivos de comunicação que se beneficiam das propriedades quânticas na última década, o que será visto em detalhes nas seções seguintes. 


\subsubsection{Protocolos de distribuição de chaves quânticas (DCQ)}

Devido a sua fundamentação em propriedades físicas, a criptografia quântica consiste em um método de distribuição de chaves criptográficas seguro mesmo quando um possível indivíduo mal intencionado possua capacidade tecnológica ilimitada, incluindo a presença de computação quântica. O primeiro protocolo de criptografia quântica foi proposto em 1984 por C. Bennett e G. Brassard (BENNETT; BRASSARD, 1984), frequentemente referenciado na literatura como BB84, e utiliza fótons polarizados como unidade básica para o estabelecimento chaves criptográficas. Esse sistema é por isso uma alternativa aos métodos criptográficos clássicos, sendo um tópico que tem atraído grande interesse nos últimos tempos.

Uma segunda família de protocolos quânticos foi apresentada em 1991, com a proposição de A. Ekert de um método utilizando as propriedades dos pares EPR apresentados na seção 3.1.1. Neste método são utilizados pares de fótons preparados inicialmente para apresentar a propriedade de emaranhamento, como é conhecido o fato de dois fótons formarem um par de Bell.

Após a proposição inicial, o desenvolvimento de protocolos de criptográfica quântica se intensificou e diversas novas propostas foram feitas. Um revisão detalhada dos protocolos de criptografia quântica existentes não é possível neste trabalho, sendo a referência (GISIN et al., 2002) uma boa fonte de consulta para isso. Contudo, o desenvolvimento de novas propostas para criptografia quântica tem sido fundamento em algumas famílias de protocolos principais, que podem ser resumidas por:

(i) BB84: utilização de fótons polarizados em duas bases ortogonais entre si, sendo estes gerados por uma das partes da comunicação e utilizados para se estabelecer uma chave e verificar a presença de espionagem;

(ii) B92: simplificação do protocolo BB84 para utilização de apenas uma base de polarização para o fóton, mantendo-se o funcionamento restante do protocolo de origem. Há ainda uma variação posterior do protocolo utilizando-se 3 bases distintas;

(iii) Protocolo EPR: protocolo que dá origem a diversos outros protocolos que em comum utilizam as propriedades de fótons emaranhados previamente preparados 
para estabelecer uma chave e verificar a presença de espionagem, diferindo dos protocolos anteriores no processo de geração dos fótons, ou seja, feito de forma conjunta por ambas as partes da comunicação;

(iii) Lo-Chau: protocolo basicamente construído sobre o protocolo EPR, utiliza a técnica de correção de erro quântica (NIELSEN; CHUANG, 2000) para obter um método de distribuição de chaves.

Apesar de ser a primeira proposta de um protocolo quântico e possuir bastante simplicidade, o protocolo BB84 tem sido um dos principais protocolos de criptografia quântica pesquisados e desenvolvidos atualmente. Os protocolos baseados em pares EPR apresentam algumas vantagens quando comparados aqueles baseados no protocolo BB84, como por exemplo a verificação de menores níveis de erro, dentre outras (GISIN et al., 2002). Contudo, devido a sua simplicidade e do desenvolvimento de técnicas capazes de implementar de forma eficiente o protocolo BB84, este têm sido o protocolo verificado nas implementação mais promissoras observadas até o momento. Por isso e visando uma abordagem voltada para aplicação prática, este trabalho tem como foco a análise e desenvolvimento de cenários de utilização do protocolo BB84 em casos práticos, apresentando e propondo algumas técnicas que podem ser utilizadas para contornar algumas das dificuldades que são encontradas em sua operação. A seção seguinte apresenta o protocolo BB84 e os principais conceitos utilizados por este no estabelecimento de uma chave criptográfica.

\subsubsection{Protocolo BB84}

O protocolo BB84 consiste em um método criptográfico de distribuição de chaves de sessão ou chaves de uso único. Devido a natureza dos sistemas quânticos, este é um método não-determinístico, não sendo possível utilizá-lo para transmitir mensagens. Contudo, o método permite estabelecer uma chave de forma segura, sendo esta utilizada em outro algoritmo simétrico de criptografia para encriptar e transmitir mensagens. É necessário para o protocolo a presença de um canal quântico e um canal público, sendo necessário que o canal quântico não deve introduzir erros acima de um limiar e sendo que o canal público deve ser autenticado, requisitos estes que serão descritos em detalhes nas seções seguintes. O protocolo permite que qualquer tentativa de espionagem seja 
detectada com alta probabilidade, e tem sua segurança formalmente demonstrada em diversos trabalhos, como aqueles que são apresentados na seção 4.1.5.

O esquema de codificação BB84 utiliza estados quânticos para codificar informação clássica, utilizando para isso os estados quânticos representados pela polarização de fótons em duas bases ortogonais, podendo os bits clássicos 0 e 1 serem representados em ambos os casos e para a qual vale o princípio da incerteza de Heisenberg e o teorema da não-clonagem, i.e., os estados não podem ser copiados sem interferência (a seção B.4 apresenta detalhes sobre a polarização da luz).

As duas bases de polarização são a base retilínea $(\oplus)$ e a base diagonal $(\otimes)$. Na base retilínea o bit 0 é associado a direção de polarização 0 , que forma o estado quântico $|0\rangle$, e o bit 1 é associado a direção de polarização $\pi / 2$, que forma o estado $|1\rangle$. Na base diagonal o bit 0 é associado a direção de polarização $+\pi / 4$, que forma o estado quântico $(|0\rangle+|1\rangle) / \sqrt{2}$ (para simplificação este será referenciado por $\left|0^{\prime}\right\rangle$, e o bit 1 é associado a direção de polarização $-\pi / 4$, que forma o estado $(|0\rangle-|1\rangle) / \sqrt{2}$ (pelo mesmo motivo, referenciado por $\left.\left|1^{\prime}\right\rangle\right)$. O esquema de bases utilizados na codificação do protocolo BB84 é mostrado na figura 16.

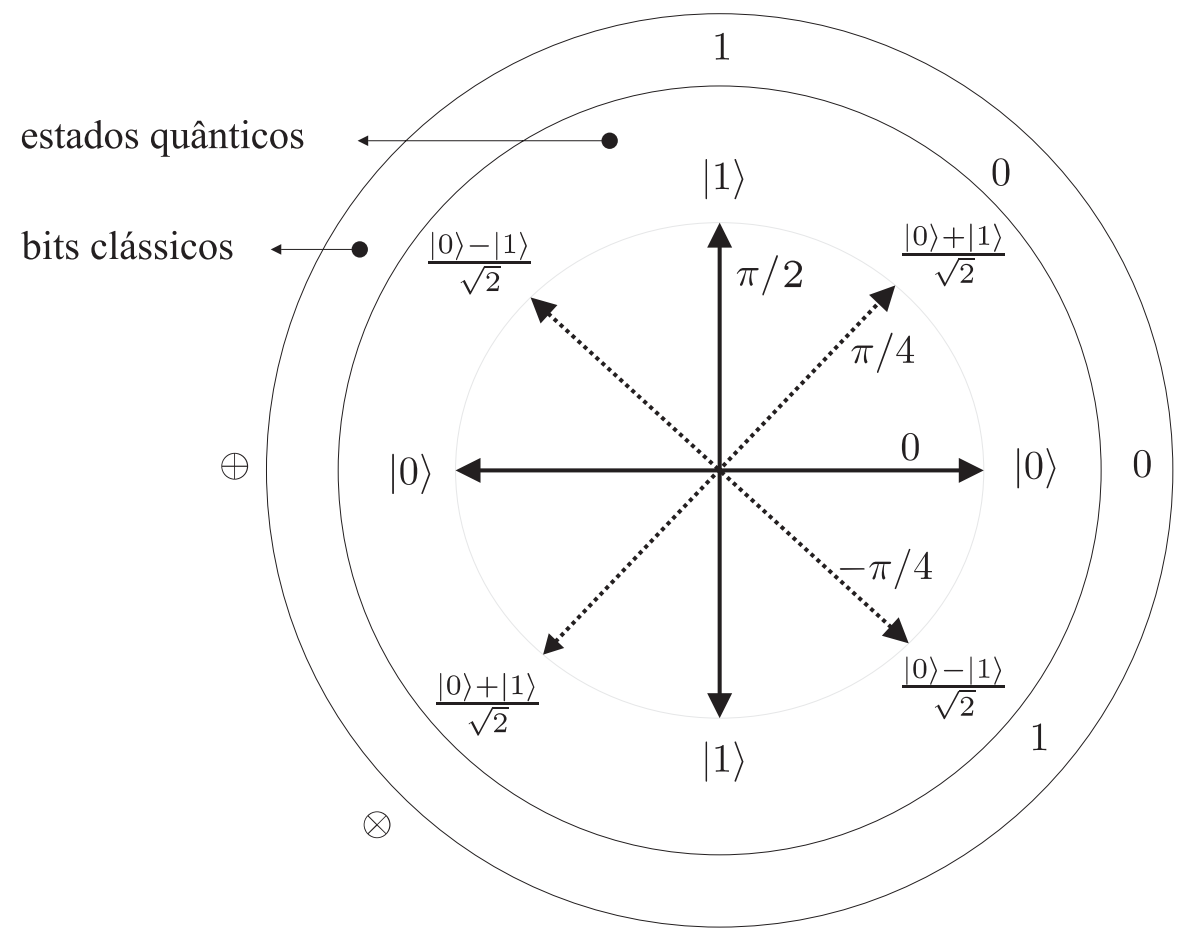

Fig. 16: Bases de codificação para o protocolo BB84 


\subsubsection{Funcionamento do protocolo BB84 na ausência de espionagem}

O processo de estabelecimento de uma chave de sessão utilizando o protocolo BB84 pode ser resumido pelos passos apresentados em seguida, que utiliza os personagens apresentados no capítulo 2 como partes que desejam estabelecer uma chave de sessão em um cenário idealista, i.e., sem ausência de ruído e tentativa de espionagem.

1. Alice gera uma sequência binária aleatória $s_{A}$ utilizando um gerador de números aleatórios. Em caráter ilustrativo, considera-se a sequência de 8 bits $s_{A}=$ [01100101].

2. Alice codifica a sequência $s_{A}$ utilizando as bases $\oplus \mathrm{e} \otimes$ (serão utilizados os dígitos 0 e 1 para as bases respectivamente) aleatoriamente, formando a sequência correspondente para polarização $m_{A}=$ [10111100]. Os qubits resultantes são então enviados a Bob por meio do canal quântico.

3. Bob recebe os qubits sem o conhecimento da sequência de bases $m_{A}$ utilizadas por Alice. Ele então escolhe aleatoriamente uma sequência de bases para realizar a medida para cada qubit enviado por Alice, utilizando por exemplo a sequência de bases $m_{B}=$ [00101010], obtendo a sequência de bits $s_{B}$. Caso a sequência de bases escolhidas por Bob corresponda a $m_{A}$, todos os bits são recuperados corretamente e $s_{B}=s_{A}$. Contudo, estatísticamente Bob utilizará a base correta em $50 \%$ das vezes, e considerando que a medição de um qubit com a base errada resultará em 0 ou 1 com $50 \%$ de chance para cada valor, a taxa de erros verificados (Quantum Bit Error Rate, QBER) entre as sequências de Alice e Bob será de $\approx 25 \%$.

4. Bob e Alice anunciam no canal público as sequência $m_{A}$ e $m_{B}$, permitindo a Bob verificar quais os bits ele obteve corretamente.

5. Por fim, Alice e Bob estabelecem os bits concordantes em $s_{A}$ e $s_{B}$ como suas chaves criptográficas $k_{A}$ e $k_{B}$, que em um cenário sem a inserção de ruído serão idênticas e terão estatísticamente o tamanho de aproximadamente $s_{A} / 2$.

A figura 17 ilustra a execução do protocolo BB84 neste caso. 


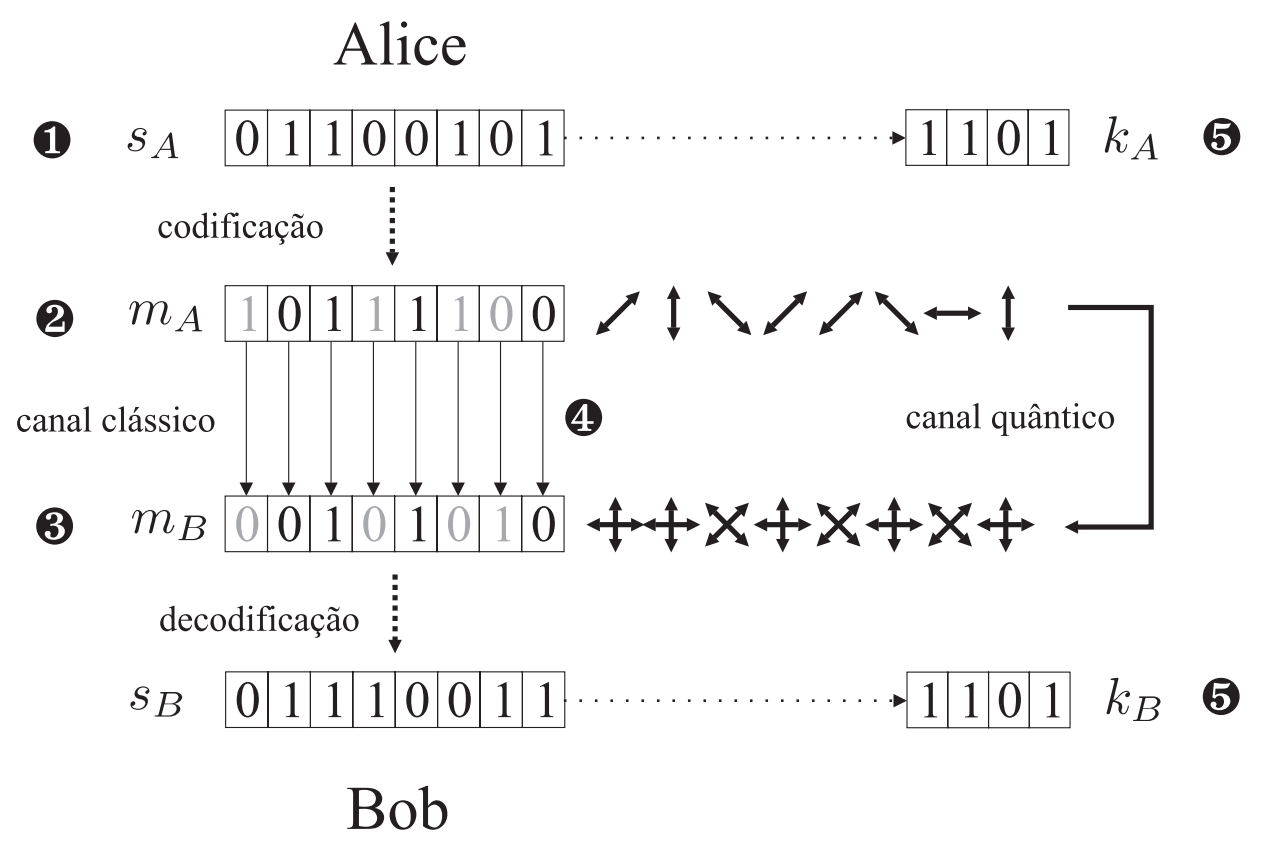

Fig. 17: Passos da execução do protocolo BB84 no caso ideal

\subsubsection{Protocolo BB84 na tentativa de espionagem}

Para um caso mais realista considera-se Eva como um indivíduo mal intencionado que deseja obter informação do canal de comunicação entre Alice e Bob. Considerando o protocolo estabelecido, Eva pode obter do canal quântico os qubits enviados por Alice com o intuito de obter a chave de sessão $k_{A}=k_{B}$ que será utilizada para a comunicação posterior. Eva não pode copiar um qubit e realizar uma medição posterior neste devido ao teorema da não-clonagem (ver seção B.6), apenas podendo realizar uma operação semelhante a que Bob executa. Como exemplo, supõe-se que Eva realize tal procedimento utilizando a sequência de bases $m_{E}=$ [10001011], obtendo a série $s_{E}=$ [01010010]. Contudo, para que esta ação não seja percebida, Eva necessita reenviar a Bob os qubits que ela realizou uma medição, lembrando que pelo postulado da mecânica quântica conhecido como colapso de superposição o qubit reenviado por Eva, uma vez medido e obtido um dado valor, resultará sempre no mesmo valor para qualquer outra medição posterior (ver seção B.3).

Como Eva não conhece a sequência de bases $m_{A}$ utilizada por Alice, ela somente pode utilizar sua sequência de bases para obter a série $s_{E}$. Estatísticamente, $m_{A}$ e $m_{E}$ são iguais para metade dos valores, implicando que $s_{E}$ terá metade dos bits em acordo com $s_{A}$. Sendo assim, o reenvio destes bits não indicará a Bob a espionagem. No entanto, 
para $25 \%$ dos bits enviados a Bob, o valor recuperado por ele estará em desacordo com aquele codificado por Alice devido a interferência de Eva. Introduzindo uma nova etapa no protocolo, onde Alice e Bob publicam e comparam entre si uma parte de $k_{A}$ e $k_{B}$, que em princípio deveriam ser idênticas, esses podem constatar a presença de espionagem por meio da existência de uma taxa de erros. A figura 18 mostra a execução do protocolo BB84 na presença de Eva.

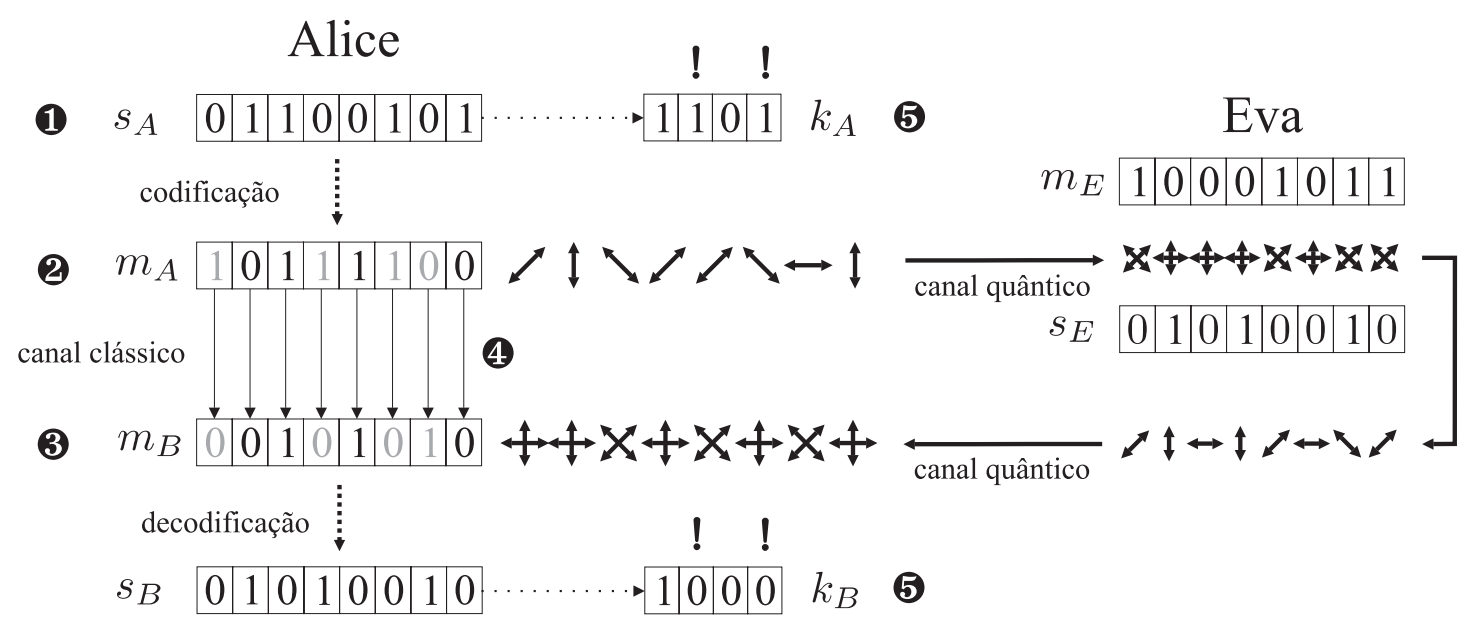

\section{Bob}

Fig. 18: Passos da execução do protocolo BB84 na presença de Eva

\subsubsection{Estimativa de erro}

Formalmente, para uma longa chave, a probabilidade de Bob receber todos os bits corretamente no caso de Eva interferir no canal é $(0,5)^{K}$, sendo $K$ o número de vezes que Eva usou a base errada. Isso se deve ao fato de que Eva, estatísticamente, escolhe a base errada em 50\% dos casos, e para uma escolha errada de base Eva recupera os valores 0 e 1 com probabilidade de $50 \%$ para ambos os casos. Esse processo gera um erro de $25 \%$, o qual permite que qualquer ação de espionagem seja invarialmente detectada por meio da comparação entre uma fração suficientemente grande das chave $k_{A}$ e $k_{B}$.

A estimativa de erro entre $k_{A}$ e $k_{B}$ pode ser feita por meio do seguinte procedimento: Alice extrai uma pequena parte da chave estabelecida por meio do protocolo e envia esta sequência a Bob. Alice informa a Bob um subconjunto de posições de tamanho $N$ e o valor do bit nessas posições na chave. Tanto Alice como Bob computam a taxa de erros $e$ observada e mantém a transmissão caso $e$ esteja abaixo de um determinado limiar 
(este valor será definido de acordo com as características de implementação utilizadas para o canal quântico), caso contrário, sendo $e$ maior que um patamar determinado a transmissão é abortada. No caso ideal, o erro verificado na ausência de Eva deveria ser nulo, caso contrário a ação de Eva tornaria $e \approx 0,25$. Contudo, no caso realista os canais de comunicação invariavelmente adicionam ruído a comunicação, sendo a análise do erro $e$ verificado neste caso é feita como proposto na seção seguinte.

\subsubsection{Protocolo BB84 em canais com ruído}

A detecção de uma tentativa de espionagem é feita no protocolo BB84 por meio da verificação da existência de uma taxa erros nos bits da chave. Contudo, uma questão importante é que, em um cenário prático, haverá sempre a inserção de erros na comunicação, representados neste caso por perdas aleatórias da polarização do fóton durante o tráfego pelo canal quântico, mesmo quando não houver tentativa de espionagem. Contudo, a distinção entre erros provocados pelo meio e aqueles oriundos de uma tentativa de espionagem pode ser realizada quando se considera que a taxa de bits errados (QBER) típicos alcançados por implementação do protocolo BB84 são menores que 5\% (GOBBY; YUAN; SHIELDS, 2007). O fato da detecção de espionagem ser feita por meio da verificação da taxa de erros verificada entre as chaves implica em um requisito geral para técnicas de implementação do protocolo BB84. Formalmente, as técnicas de implementação devem possuir uma taxa de geração de erros abaixo do limiar de 25\%, taxa que não indica uma tentativa de espionagem. Em casos práticos, para evitar falsas detecções de tentativa de espionagem, é exigido uma taxa de erros não superior a $10 \%$ (GOBBY; YUAN; SHIELDS, 2007).

Uma cenário ainda mais realista é representado pela situação em que Eva obtém apenas uma pequena fração da chave de sessão. Na situação apresentada anteriormente Eva interfere no canal quântico e obtém $50 \%$ da informação transmitida, gerando uma QBER de $\approx 25 \%$. Se Eva, contudo, interferir em apenas 1/10 dos qubits enviados por Alice, sua ação irá gerar uma QBER de apenas $\approx 2,5 \%$, valor que pode se passar por ruído. Neste caso sua presença não será detectada como uma tentativa de espionagem, sendo que Eva obtém apenas $\approx 5 \%$ da informação transmitida por Alice. Dessa forma Eva terá uma sequência, que mesmo fracamente, estará correlacionada à chaves $k_{A}=k_{B}$ 
de Alice e Bob. Para tornar o método mais eficiente, dois procedimentos são realizados com a sequência de bits da chave de sessão: os procedimentos de reconciliação de informação e amplificação de privacidade. O processo de reconciliação de informação executa a correção de erros para as sequências de Alice e Bob e o processo de amplificação de privacidade aumenta a correlação entre as sequências de Bob e Alice enquanto diminui a correlação entre a sequência de Eva. Este procedimentos são fundamentais para a inserção do protocolo de criptografia quântica BB84 em cenários práticos, tendo seu funcionamento detalhado nas seções seguintes.

\subsubsection{Reconciliação de informação}

O procedimento de reconciliação de informação é um processo de correção de erros interativo que é feito sobre o canal clássico com o intuito de diminuir a diferença entre as sequência de Alice e Bob causada pela existência de uma taxa geração de erros no canal quântico. As técnicas de correção de erros conhecidas exigem a manipulação de parte da informação a ser corrigida, o que no caso do protocolo quântico implica no anúncio dessa informação no canal público. Esse fato implica que Eva ganhará alguma informação referente a chave de sessão no processo de correção de erros. Assim, o protocolo BB84 exige que na etapa de reconciliação de informação seja utilizado um método de correção de erros que necessite do mínimo de divulgação de informação. Algumas técnicas foram propostas desde o surgimento do protocolo BB84, sendo algumas discutidas em (GISIN et al., 2002). Contudo, a técnica que apresenta os melhores resultados e vem sendo usada como referência na implementação de protocolos de criptografia quântica consiste no chamado protocolo Cascade, apresentado inicialmente em (BENNETT et al., 1992). O protocolo utiliza a divulgação de alguns bits de paridade relacionados a subconjuntos da chave de sessão estabelecida pelo protocolo BB84 em um processo interativo que corrige progressivamente os erros encontrados. Neste ponto, o presente trabalho realiza uma contribuição original no desenvolvimento de uma nova abordagem na correção de erros, baseada no protocolo Cascade, que será por isso chamado de protocolo Cascade Modificado. Esta nova proposição utiliza alguns dos conceitos básicos apresentados em (BENNETT et al., 1992), os quais têm sua eficiência discutida e demonstrada em (BRASSARD; SALVAIL, 1994), para obter uma versão mais eficiente do algoritmo Cascade. 
A proposta original representada pelo protocolo Cascade Modificado utiliza interações por meio do canal público para corrigir eficientemente os erros presentes na chave de sessão compartilhada pelo protocolo BB84 por meio da divisão desta em blocos de tamanho fixo, que são submetidos a algumas primitivas interativas descritas a seguir. O protocolo opera de forma que para uma sequência de bits de tamanho $n$, divida em blocos de tamanho $k$, e com probabilidade de se encontrar erros dada por $\sigma_{k}$, a informação divulgada na execução do protocolo é proporcional a $\sigma_{k}$ e $1 / k$.

BINARY Primitiva que realiza para duas sequências $A$ e $B$, associadas as chaves de sessão de Alice e Bob e que possuam um número ímpar de erros, uma busca binária de forma a encontrar os erros revelando não mais que $\lceil\log n\rceil$ bits sobre o canal público. A primitiva opera da forma:

1. Alice envia a Bob a paridade da primeira metade de sua sequência;

2. Bob determina se há um número ímpar de erros na metade inicial de sua sequência testando a paridade desta e comparando o valor com a informação enviada por Alice;

3. Este procedimento é repetido para o subconjunto subsequente, i.e., a metade inicial ou final, em que há um número ímpar de erros, até que um erro seja encontrado.

CONFIRM Se as sequências de Alice e Bob são diferentes, esta primitiva indica o fato com probabilidade $1 / 2$, caso contrário, se as sequências são idênticas, a primitiva indica o fato com probabilidade 1 por meio do procedimento:

1. Alice e Bob selecionam aleatoriamente um subconjunto de bits correspondentes de suas sequências;

2. Alice informa a Bob a paridade de seu subconjunto;

3. Bob verifica se seu subconjunto tem mesma paridade. O processo pode ser repetido $k$ vezes para aumentar sua precisão, sendo que este teste falhará com probabilidade $2^{-k}$.

BICONFIRM $^{s}$ Combinação das primitivas anteriores gerando uma nova primitiva capaz de corrigir vários erros. A primitiva $\mathrm{BICONFIRM}^{s}$ executa CONFIRM $s$ vezes. A 
cada execução de CONFIRM é testado a paridade de um subconjunto aleatório das sequências de Alice e Bob, e caso estas sejam diferentes é executada a primitiva BINARY para o subconjunto em questão. Seja $\Delta^{s}(l \mid e)$ a probabilidade da primitiva BICONFIRM $^{s}$ corrigir $l$ erros se há $e$ erros. Tem-se então que:

$$
\Delta^{s}(l \mid e)= \begin{cases}\left(\begin{array}{l}
s \\
l
\end{array}\right) 2^{-s} & \text { se } l \neq e \\
\sum_{j=e}^{s}\left(\begin{array}{l}
j-1 \\
e-1
\end{array}\right) 2^{-j} & \text { se } l=e\end{cases}
$$

Utilizando as primitivas anteriores é possível obter um protocolo interativo de correção de erros com número de passos determinado. O protocolo Cascade Modificado é executado por Alice e Bob simultaneamente, com um número de passos previamente definido, operando sobre as chaves de sessão de Alice e Bob, $A=\left(A_{1}, \ldots, A_{n}\right)$ e $B=\left(B_{1}, \ldots, B_{n}\right)$ respectivamente, com $A_{i}, B_{i} \in\{0,1\}$. O primeiro passo do protocolo Cascade Modificado consiste na escolha conjunta de Alice e Bob de $k_{1}$ e a divisão de suas sequências em blocos de tamanho $k_{1}$. Alice informa então a paridade de cada um dos seus blocos a Bob, que compara essa informação com a paridade dos blocos de sua sequência. Utilizando a primitiva BINARY, Alice e Bob corrigem os erros dos blocos para os quais foi detectado uma paridade diferente e após isso eliminam o último bit de cada bloco. Este processo é repetido nos passos seguintes com $k_{i+1}=2 k_{i}$ enquanto $k_{i+1}<n / 4$. Neste ponto todos os blocos de Bob terão um número par de erros, incluindo a possibilidade de existir zero erros. O passo seguinte consiste na execução da primitiva BICONFIRM $^{s} s$ vezes utilizando um bloco de tamanho $k=n / 4$ e excluindo a cada verificação de paridade diferente o último bit do bloco corrigido. O número de execuções $s$ é definido previamente por Alice e Bob, sendo sua escolha associada ao nível de segurança desejado. A escolha do tamanho do bloco inicial $k_{1}$ e o número de execuções $s$ é empírica, sendo apresentado na seção 7.1.1, que mostra o desenvolvimento de um ambiente de simulação para o protocolo BB84 utilizando o protocolo Cascade Modificado na fase de reconciliação de informação e algumas formas de definir valores eficientes para esses parâmetros.

O algoritmo Cascade Modificado permite a correção de forma eficiente dos erros presentes nas sequências de Alice e Bob. Contudo, a divulgação dos valores de paridade realizada durante a execução do protocolo fornece alguma informação a Eva sobre a 
chave estabelecida entre Alice e Bob, mesmo com a eliminicação de alguns bits dos blocos testados. A utilidade dessa informação do ponto de vista de Eva é discutida na seção seguinte, juntamente com a definição de um mecanismo que permite tornar inútil a informação capturada por Eva.

\subsubsection{Amplificação de privacidade}

A reconciliação de informação permite a Alice e Bob estabelecer uma chave idêntica, mas apenas parcialmente secreta. Isso porque a informação divulgada na execução do protocolo de correção de erros confere a Eva alguma informação a cerca da chave estabelecida. Adicionalmente a informação que Eva obtém do processo de reconciliação de informação, esta pode ganhar alguma informação extra a partir de vulnerabilidades conhecidas de algumas técnicas utilizadas na implementação de protocolos quânticos, sendo estas discutidas em detalhes na seção 4.1.3.

Entretanto, a técnica conhecida como amplificação de privacidade, introduzida inicialmente em (BENNETT; BRASSARD; ROBERT, 1988), permite tornar inútil a informação que Eva obtém a cerca da chave, considerando as diferentes fontes de vazamento de informação disponíveis. Alice e Bob podem estimar, por meio de um procedimento detalhado na seção 4.1.4, um inteiro $l$ tal que a informação obtida por Eva a partir do canal quântico não é maior que $l$ bits da chave estabelecida. A informação revelada durante a execução do protocolo Cascade Modificado refere-se apenas à paridade de blocos da chave, sendo que em cada etapa do protocolo um bit é excluído do bloco corrigido. Essa informação permite a Eva obter a partir do conhecimento de bits físicos, i.e., informação adquirida por algum outro método, informação sobre a paridade de algumas partes da chave estabelecida. É possível verificar que se Eva não conhece mais que $l$ bits de paridade a cerca de uma sequência $y$, e que se a ela é dado um bit de paridade adicional a cerca de $y$, e sendo $z$ a sequência formada a partir do descarte de algum bit que influencia na paridade adicional obtida por Eva, então Eva continuará após a aquisição da paridade adicional não conhecendo mais que $l$ bits de paridade a cerca de $z$. Portanto, se Eva não conhece mais que $l$ bits físicos da chave antes do processo de reconciliação de informação, mesmo com a divulgação de informação sobre a paridade de alguns blocos seguido do descarte de alguns bits, Eva não conhecerá mais que $l$ bits de paridade a 
cerca da sequência estabelecida após este processo (BENNETT et al., 1995).

Diante disso, o procedimento de amplificação de privacidade pode ser fundamentado na seguinte propriedade apresentada em (BENNETT; BRASSARD; ROBERT, 1988), conhecida como como teorema da amplificação de privacidade que é definido a seguir.

Teorema 4.1.1. Dada uma sequência de bits de tamanho n compartilhada entre Alice e Bob e sendo um bit determinístico de informação a cerca de x o valor e $(x)$ de uma função arbitrária e $:\{0,1\}^{n} \rightarrow\{0,1\}$. Então se a informação conhecida por Eva a cerca de $x$ não é maior que l bits determinísticos, uma função de hash $h(x)$ aleatória e publicamente escolhida a partir da classe de funções de Hash universal ${ }_{2}$, com $h(x)$ : $\{0,1\}^{n} \rightarrow\{0,1\}^{n-l-s}$, irá mapear x em $h(x)$ tal que a informação que Eva possuíra após isso será menor que $2^{s} / \ln 2$ bits, sendo $s>0$ um parâmetro de segurança arbitrário.

O teorema anterior se aplica a informação divulgada no processo de reconciliação de informação realizado por Alice e Bob uma vez que bits físicos e de paridade são casos especiais de bits determinísticos. O papel do parâmetro de segurança $s$ e uma forma de definí-lo serão discutidos posteriormente.

Uma função de hash que pode ser utilizada no procedimento de amplificação de privacidade consiste no cálculo da paridade para diferentes subconjuntos da chave estabelecida após o processo de reconciliação aleatoriamente definido e tendo tamanho $n-l-s$, mas neste caso retendo estes valores de paridade e formando, a partir desta, a chave final do processo. Uma função de hash construída dessa forma faz parte da classe pertence a classe de funções de Hash universal ${ }_{2}$, sendo formalmente um elemento da classe $\mathrm{H} 3$ fortemente-universal 2 , como demonstrado em (WEGMAN; CARTER, 1981), Neste caso é então válido o teorema da amplificação de privacidade apresentado no parágrafo anterior.

É importante ressaltar que a execução do processo de amplificação de privacidade como definido anteriormente implica que se houver ao menos um erro entre as sequências de Alice e Bob após o processo de reconciliação de informação, então a chave final obtida será completamente descorrelacionada. Assim, o número de interações realizadas no processo de reconciliação de informação deve ser adequadamente definido de forma que a chave obtida após este processo tenha alta probabilidade de ser idêntica. A 
seção 7.1.1 mostra como esta escolha pode ser feita utilizando o exemplo do estabelecimento de comunicação em um caso prático.

O processo de amplificação de privacidade permite então a Alice e Bob obterem uma chave final idêntica e secreta, considerando que Eva após o processo conhecerá não mais que $2^{s} / \ln 2$ bits, sendo que o parâmetro $s$ pode ser adequadamente ajustado para que Eva não conheça nenhuma informação sobre a chave final. O procedimento, contudo, envolve a obtenção de uma estimativa da informação que Eva pode capturar por meio de mecanismos de vazamento de informação. Uma destes mecanismos consiste, como foi visto, na divulgação de informação durante o procedimento de reconciliação de informação. Outros mecanismos estão relacionados a algumas técnicas de ataque que Eva pode executar contra o protocolo quântico, sendo estes detalhados na seção seguinte. A estimativa da informação que Eva possui pode então ser feita a partir do cálculo da informação vazada por cada um dos métodos de ataque disponíveis a Eva, como mostrado na seção 4.1.4.

\subsubsection{Ataques contra o protocolo BB84}

Apesar dos conceitos apresentados anteriormente indicarem que o protocolo BB84 é seguro em seu fundamento teórico, imperfeições que ocorrem invariavelmente na implementação do protocolo permitem que alguns ataques possam ser efetuados explorando certas vulnerabilidades. As principais técnicas de ataques conhecidos são discutidas a seguir.

\subsubsection{Ataque man-in-the-middle}

O protocolo BB84 se torna totalmente vulnerável quando considerado o problema da autenticação do canal clássico. Em um cenário prático, onde o protocolo deve ser executado através de longas distâncias, é necessário um mecanismo que permita a Alice e Bob se certificarem de estarem se comunicando com quem esperam. A falta de tal mecanismo permite a Eva agir entre as partes da comunicação se fazendo passar por Bob para Alice e se passando por Alice para Bob. Ou seja, nesse caso Eva estabeleceria as chaves da comunicação com cada uma das partes sem que ela fosse notada. 
Uma das formas de contornar essa vulnerabilidade é a utilização de alguma técnica de autenticação das partes da comunicação, impedindo com isso que Eva se passe ou por Alice ou por Bob. Na proposta original de Charles Bennett (BENNETT; BRASSARD, 1984) é apresentado como sugestão para o problema da autenticação a técnica desenvolvida por Wegman e Carter (NAOR; YUNG, 1989), que se baseia na utilização de uma função da classe das funções de Hash. Contudo, este método exige que uma pequena informação secreta seja estabelecida previamente por Alice e Bob para a escolha da função de Hash a ser utilizada. Pela natureza das funções Hash, a probabilidade de se deduzir a correspondência para a função sem conhecer essa informação prévia é extremamente baixa mesmo para capacidade computacional ilimitada, incluindo computação quântica.

A solução proposta no trabalho original mantém a segurança do protocolo, contudo implica no fato que o protocolo BB84 se comportaria como um mecanismo seguro de expansão de chaves a partir de um segredo prévio. Em alguns cenários, esta é uma utilização bastante útil, uma vez que em certos casos é possível as entidades da comunicação estabelecerem um segredo prévio por meio do encontro físico, mantendo comunicação remota segura indefinidamente após isso por meio da expansão desse segredo original utilizando o protocolo BB84.

Há casos, entretanto, que o estabelecimento de uma informação prévia é inviável, sendo este um dos principais motivadores para o desenvolvimento das técnicas de criptografia de chave pública apresentadas na seção 2.3. Uma alternativa para o problema de autenticação no protocolo BB84 poderia ser a utilização de algum dos métodos de autenticação discutidos no capítulo 2. Contudo, essa alternativa tornaria o protocolo BB84 tão vulnerável quanto os protocolos clássicos, uma vez que uma falha de autenticação no protocolo o torna totalmente vulnerável pelos motivos discutidos anteriormente.

Não há ainda uma solução definitiva para este problema. Contudo, diversas abordagens utilizando propriedades quânticas na obtenção de um método de autenticação mais seguro têm sido feitas, sendo que algumas propostas utilizando sistemas capazes de processar informação quântica são conceitualmente eficientes nessa tarefa, como aquelas apresentadas em (ZENG; KEITEL, 2002; WANG; ZHANG; TANG, 2006). Essas abordagens necessitam, entretanto, da disponibilidade da implementação de computação quântica, 
o que torna a utilização da técnica mais difícil em um prazo próximo.

Neste trabalho é apresentada uma proposta adicional para a realização da autenticação do canal clássico no protocolo BB84, representada por um mecanismo de autenticação baseado em propriedades quânticas e compatível com o protocolo BB84. Este não necessita de processamento de informação quântica, mas tal como outros métodos clássicos necessita do compartilhamento de um pequeno segredo prévio. Esse método de autenticação será apresentado em detalhes sna seção 4.1.6.

\subsubsection{Interceptação-reenvio}

Essa é a estratégia de ataque mais acessível a Eva para tentar corromper o protocolo BB84. Nesse ataque Eva recebe os qubits provindos de Alice e realiza uma medição em uma das duas bases de medida do protocolo BB84, tal como faria Bob. Ela então prepara um qubit no estado igual ao medido por ela e então envia este a Bob. Eva tem uma chance de $50 \%$ de realizar a medição correta para o qubit enviada por Alice. Neste caso ela também enviará o estado correto a Bob, não sendo assim detectada. Contudo, para $\approx 50 \%$ da informação Eva vai causar uma descorrelação entre os resultados de Alice e Bob, o que ajudará Alice e Bob a detectar sua presença. Isso permite a Eva capturar $\approx$ $50 \%$ da informação referente a chave trocada por Alice e Bob, produzindo um aumento na taxa de erros de $\approx 25 \%$. Contudo, para não ser detectada e tentar confundir os erros causados por sua presença com o erro intrínseco ao canal quântico, Eva aplica o ataque a apenas uma fração dos qubits enviados por Alice, por exemplo 20\%, gerando uma taxa de erro de somente $\approx 5 \%$, e obtendo cerca de $10 \%$ da informação acerca da chave trocada.

Por meio da estimativa da informação que Eva captura por meio desse ataque e dos demais ataques conhecidos é possível realizar o procedimento de amplificação de privacidade apresentado anteriormente de forma que a informação obtida por Eva seja tão pequena quanto desejado. A informação obtida pela técnica de interceptação/reenvio pode ser obtida da forma: se há $t$ erros produzidos pelo canal quântico, então Alice e Bob podem estimar de forma conservadora que não mais que $4 t+5 \sqrt{12 t}$ dos seus bits foram submetidos a uma ação de interceptação/reenvio, sendo o segundo termo da expressão referente um desvio-padrão igual a 5 considerado devido a possíveis erros estatísticos. 
Com isso, Alice e Bob podem estimar que no pior caso que não mais que $\xi$ bits, tal que:

$$
\xi=(4 / \sqrt{2}) t+5 \sqrt{(4+2 \sqrt{2}) t}
$$

foram capturados por Eva por meio desse tipo de ataque, estimativa baseada nos argumentos apresentados na referência (BENNETT et al., 1991). Considerando que erros na prática podem ser gerados por diversas fontes, a estimativa anterior é bastante conservadora uma vez que considera que todos os erros são devidos a uma ação de interceptação/reenvio. Isso torna a estimativa mais segura.

\subsubsection{Ataque beam-splitting}

Outra fonte de obtenção de informação consiste em um ataque que utiliza a imperfeição das implementações físicas do canal quântico para tentar extrair informação acerca da chave trocada por meio do protocolo. Frequentemente, os geradores de pulso de laser utilizados nas implementações do protocolo BB84 não produzem apenas um fóton por pulso, ocorrendo ocasionalmente a produção de mais de um fóton em cada pulso. Esse fóton em excesso pode ser utilizado por Eva por meio de uma técnica de divisão de feixe, o que permite que ela possa realizar uma medição sobre um dos fótons deixando inalterado o outro que é então enviado a Bob. A presença de Eva nesse caso é difícil de ser detectada uma vez que ela não altera o estado dos qubits que chega a Bob. Contudo, nas fontes de fótons atuais, a produção de mais de um fótons por pulso é marginal, tendo uma distribuição de probabilidade tipicamente equivalente a distribuição de Poisson (ver apêndice B.4.1). Isso permite que a informação marginal adquirida por Eva por meio de um ataque de beam-splitting possa ser adequamente tratada no processo de amplificação de privacidade. A informação obtida por Eva por meio desse ataque pode ser estimada da forma: considerado uma probabilidade $\mu$ de ocorrência de fótons por pulso, então a informação adquirida por Eva por meio deste ataque para $N$ pulsos recebidos corretamente por Bob, i.e., medidos na base correta, pode ser de forma conservadora dada por $\sigma$ bits, tal que:

$$
\sigma=N \mu+5 \sqrt{N \mu(1-\mu)}
$$


O segundo termo da equação (4.3) refere-se a permissão de um desvio-padrão igual a 5, relacionado a consideração do pior caso, quando Eva tem mais sorte que a média na execução de divisões de feixe.

\subsubsection{Ataques recentes}

Recentemente foi proposta uma nova técnica de ataque ao protocolo BB84. Esta utiliza um circuito quântico determinístico que opera sobre dois qubits presentes em um único fóton. A proposta utiliza a característica de emanharamento e algumas das previsões teóricas do que se conhece como informação de Rényi para capturar informação da chave trocada por Alice e Bob a partir da existência de erros no canal quântico. Um ambiente de simulação para a técnica foi apresentado em (KIM et al., 2007), demonstrando que a técnica é válida e permite atacar o protocolo BB84.

Apesar da grande atenção que este trabalho atraiu, indicando que a criptografia quântica poderiam ser corrompida, a técnica se aplica somente em um caso bastante específico. Não cabe aqui a realização de uma descrição detalhada do procedimento. Contudo, uma consideração importante feita no ambiente de simulação proposto em (KIM et al., 2007) deve ser considerada. A aplicação da técnica se baseia no fato que Eva tenha acesso físico ao módulo receptor de fótons de Bob. Esta é uma condição que não se aplica a um caso prático, uma vez que possibilidade que Eva tenha acesso físico ao aparato de Bob cria uma vulnerabilidade que vai além das propriedades da mecânica quântica.

Assim, a técnica de ataque proposta contribui na investigação dos limites fundamentais da segurança no protocolo BB84 na presença de espionagem e erros físicos realistas. Adicionalmente, cria uma situação adicional que deve ser considerada no desenvolvimento de técnicas para os procedimentos de reconciliação de informação e amplificação de privacidade. Dessa forma, este resultado não inviabiliza o protocolo BB84 tal como as técnicas desenvolvidas anteriormente. 


\subsubsection{Estimativa da informação adquirida por meio de técnicas de ataque}

A realização do procedimento de amplificação de privacidade descrito anteriormente necessita do cálculo de uma estimativa da informação capturada por Eva na execução do protocolo. Essa estimativa é baseada na informação que é divulgada durante a execução do protocolo e na informação que pode ser capturada pelos ataques de beamsplitting e interceptação-reenvio. Apenas estes ataques são considerados uma vez que estes permitem a obtenção marginal de informação a cerca da chave, sendo estes as alternativas desse tipo viáveis em um caso prático. O ataque man-in-the-middle corrompe completamente o protocolo BB84, sendo considerada outra forma de proteção, como discutido anteriormente.

A fração de informação da chave estabelecida por Alice e Bob que é adquirida por meio deos ataques de beam-splitting e interceptação-reenvio é limitada superiormente por $\rho$, tal que:

$$
\rho=\mu+(4 / \sqrt{2}) e,
$$

com $\mu$ sendo a intensidade média do feixe de fótons, equivalente a probabilidade de ocorrência de fótons por pulso, e $e$ a taxa de erros no canal quântico, i.e., $e=t / N$. A informação obtida por Eva em ambos tipos de ataque é correlacionada, como mostrado em (BENNETT et al., 1991). Isso implica que quantidade de informação adquirida por Eva é menor que a soma das estimativas definidas nas equações (4.2) e (4.3). Contudo, considerando novamente o pior caso e a partir das equações (4.2) e (4.3), a informação adquirida por Eva em ambos os tipos de ataque pode ser conservadoramente dada por $l$, tal que:

$$
l=N \rho+5 \sqrt{N(\mu(1-\mu)+(4+2 \sqrt{2}) e)}
$$

com $N$ sendo o tamanho da sequência inicial após o anúncio de bases, $\mu$ a intensidade média do feixe de fótons e $e$ a taxa de erros no canal quântico. O segundo termo na equação refere-se a consideração de um desvio-padrão igual a 5. 


\subsubsection{Provas de segurança do protocolo $\mathrm{BB84}$}

Um protocolo de criptografia é dito incondicionalmente seguro quando é possível provar matematicamente que, uma vez atendido os requisitos de operação do protocolo, este é seguro para qualquer estratégia de ataque. Este é o caso por exemplo da Cifra de Vernam com demonstração formal feita por Claude Shannon.

O protocolo BB84 é referenciado frequentemente como um protocolo de criptografia incondicionalmente seguro. Contudo, o protocolo BB84 é na verdade probabilísticamente seguro, sendo possível tornar a probabilidade de falha deste assintoticamente tendendo a zero. Mesmo com a existência de algumas técnicas de ataque conhecidas, como aquelas mostradas nas seções anteriores, a segurança da operação do protocolo BB84, quando atendido alguns requisitos de operação, pode ser demonstrada formalmente. Isto significa que o protocolo é assintoticamente seguro contra todos os ataques permitidos pela mecânica quântica.

As demonstrações formais de segurança do protocolo BB84 são extensas e complexas, requerendo a apresentação de conceitos que não fazem parte do escopo deste trabalho. As principais demonstração de segurança realizadas para o protocolo BB84 foram feitas por D. Mayers (MAYERS, 1998), W. Shor e J. Preskill (SHOR; PRESKILL, 2000), D. Gottesman e H.K Lo (GOTTESMAN; LO, 2003), podendo ser consultadas detalhadamente nas referências indicadas.

\subsubsection{Proteção contra ataques man-in-the-middle}

Nesta seção é apresentada uma proposta original adicional para a realização da autenticação do canal clássico no protocolo BB84. Esta se fundamenta nas propriedades da mecânica quântica e no próprio protocolo BB84. O método original que é proposto neste trabalho, o qual será chamado de BB84-A, utiliza um processo similar àquele envolvido no protocolo BB84 para realizar a autenticação das partes da comunicação, considerando o compartilhamento prévio de um par de chaves preposicionadas $k_{a}=k_{b}$. $\mathrm{O}$ protocolo BB84-A proposto é descrito pelos passos seguintes, sendo utilizada a mesma terminologia do protocolo BB84 apresentada anteriormente:

1. Alice gera um sequência de bits aleatória; 
2. Alice codifica cada um dos bits presentes em um qubit de acordo com a escolha de uma das bases diagonal ou retilínea $\{\oplus, \otimes\}$ e envia estes a Bob;

3. Bob escolhe uma base aleatoriamente dentre as duas possibilidades $\{\oplus, \otimes\}$ e realiza a medida sobre cada um dos qubits recebidos;

4. Bob armazena as escolhas de bases que realizou por meio de uma sequência de bits $b_{b}$ utilizando 0 para a base $\oplus$ e 1 para a base $\otimes$. Após isso, Bob encripta sua sequência por meio da Cifra de Vernam com uma chave preposicionada $k_{b}$, e envia essa sequência a Alice usando o canal clássico;

5. As bases escolhidas por Alice para a codificação dos qubtis são armazenadas em uma sequência $b_{a}$, utilizando 0 para a base $\oplus$ e 1 para a base $\otimes$. Após isso, Alice encripta sua sequência por meio da Cifra de Vernam com uma chave preposicionada $k_{a}$, e envia essa sequência a Alice usando o canal clássico;

6. Alice e Bob realizam a desencriptação de suas bases utilizando sua chave preposicionada e a sequência recebida da contra-parte, obtendo em ambos os casos $\left(b_{a} \oplus b_{b}\right)$. Ambos então descartam todas as posições $i$ onde $b_{a}[i] \oplus b_{b}[i]=1 \mathrm{e}$ estabelecem o resultado como duas novas sequências $x_{a}$ e $x_{b}$;

7. Alice e Bob podem então comparar a sequência de bits destilada a partir de $x_{a}$ e $x_{b}$ por um processo equivalente aquele discutido na seção 4.1.2 para detectar a presença de Eva ou validar a autenticação.

Com isso, o algoritmo permite, a partir da troca de um segredo prévio representado pelo par de chaves $k_{a}=k_{b}$, a autenticação das partes da comunicação no protocolo BB84. Contudo, a necessidade do compartilhamento prévio de informação não constitui uma solução definitiva. Nesse sentindo, o protocolo BB84-A se iguala à outras técnicas clássicas existentes, mas apresentando o benefício da realização da autenticação com os mesmos equipamentos e mecanismos utilizados no protocolo BB84.

Em alguns casos o compartilhamento de um pequeno segredo prévio não constitui um grande obstáculo. Adicionalmente, o protocolo BB84-A exige a utilização deste segredo apenas no estabelecimento da comunicação, podendo ser as chaves de autenticação $k_{a}=k_{b}$ obtidas da própria operação do protocolo BB84. 
O cenário anterior indica que a proposta do protocolo BB84-A pode ser relevante em alguns casos práticos. Contudo, essa necessita ainda de uma prova formal de sua segurança e uma avaliação de sua utilização em um ambiente prático.

\subsection{Protocolos quânticos e redes de comunicação}

O desenvolvimento teórico apresentado nas seções anteriores evidencia que a utilização de protocolos de criptografia quântica, especialmente aqueles derivados do protocolo BB84, são, conceitualmente, uma alternativa para futuras gerações de sistemas criptográficos resistentes a tecnologias de processamento de informação avançadas. Contudo, algumas questões fundamentais devem ser consideradas para a utilização prática de protocolos de criptografia quântica como mecanismo de segurança da informação operando em uma típica infra-estrutura de telecomunicações atual.

Um primeiro aspecto importante refere-se ao fato de que os protocolos de criptografia quântica operam essencialmente em uma comunicação do tipo ponto-a-ponto entre dois usuários, o que restringe bastante sua aplicabilidade em diversos dos cenários frequentemente observados em redes de comunicação. Um segundo aspecto que decorre diretamente do anterior é representado pelo fato que protocolos de criptografia quântica estão sujeitos a uma distância e taxa de transferência máximas no processo de comunicação. Por fim, um terceiro aspecto consiste em como obter uma estrutura de telecomunicações utilizando protocolos quânticos, o que vem sendo chamado de rede quântica, que possa superar as limitações envolvendo a comunicação ponto-a-ponto, permitindo a comunicação entre grandes distâncias e múltiplos usuários de forma interoperável com a infra-estrutura de telecomunicações já existente.

Muito tem sido feito nos últimos anos na tentativa de tratar esses aspectos e levar as técnicas de criptografia quânticas a realidade de aplicações práticas. O trabalho de diversos institutos de pesquisa privados, governamentais e acadêmicos tem apresentado relevantes avanços. Grandes corporações da indústria de tecnologia como IBM, HP, NEC, dentre outras, possuem laboratórios ativos e orçamentos dedicados ao tema. Adicionalmente, importantes contribuições têm sido feitas por institutos governamentais, especialmente o National Institute of Standards and Technology (NIST) do governo 
norte-americano, empresas financiadas com recursos governamentais como BBN Technologies e QinetiQ, além de start-ups como MagiQ e IdQuantique, sendo estas últimas responsáveis pelos primeiros dispositivos comerciais de criptografia quântica. O trabalho conjunto desenvolvido por estas diversas entidades já apresenta alguns avanços práticos que podem ser vistos por meio de dois grandes projetos que vêm sendo executados com o objetivo de obter e manter em operação uma rede quântica: DARPA Quantum Network, projeto conjunto coordenado pela Defense Advanced Research Projects Agency (DARPA) com contribuições das empresas BBN Technologies e QnetiQ e do NIST (ELLIOT, 2002); e o projeto SECOQC, consórico entre diversos institutos de pesquisa acadêmica europeus e empresas como a idQuantique (SECOQC, 2007).

O projeto desenvolvido pela agência americana DARPA consiste na obtenção de uma rede de distribuição de chaves criptográficas por meio de protocolos quânticos entre alguns pontos localizados na região metropolitana de Boston, EUA. O projeto, que está em operação desde 2003, consiste em canais de comunicação terrestres sendo recentemente incorporado um canal de comunicação por espaço aberto por meio de uma abordagem desenvolvida em conjunto com a empresa QinetiQ e o NIST.

O projeto SECOQC foi idealizado como um projeto de longo prazo no âmbito do programa de financiamento cientifico da comunidade européia Framework Programme envolvendo diversas instituições acadêmicas e algumas empresas com o objetivo de desenvolver e implementar uma rede quântica de distribuição de chaves unificada para a comunidade européia incluindo canais de comunicação terrestres e por espaço aberto. Alguns desdobramentos importantes resultaram deste projeto, como a utilização da tecnologia de criptografia quântica desenvolvida pela idQuantique, uma das principais parceiras do projeto SECOQC, nas eleições suíças de outubro de 2007 (MARKS, 2007).

As tecnologias de implementação de protocolos de criptografia quântica se dividem em duas classes: a transmissão de fótons por meio de fibras ópticas e a transmissão de fótons por espaço aberto. A pesquisa envolvendo técnicas de implementação de protocolos quânticos constituem um extenso tópico do campo geral de criptografia quântica, envolvendo diversas áreas do conhecimento, desde eletrônica a ciência dos materiais. Não é possível realizar um pesquisa detalhada deste tópico no presente trabalho, sendo, entretanto, realizado um breve resumo de cada abordagem nas seções seguintes. A partir 
desse resumo é possível discutir as principais dificuldades técnicas relacionadas a cada caso e o impacto que a utilização da abordagem tem na obtenção de uma rede quântica de troca de chaves criptográficas de escala global.

\subsubsection{Criptografia quântica em comunicações por fibra óptica}

A implementação de protocolos de criptografia quântica por meio da transmissão de fótons por fibra óptica é uma abordagem imediata quando se considerada a extensa infra-estrutura de fibras ópticas em operação atualmente, o grande desenvolvimento da tecnologia de fabricação de fibras e fontes de luz como LEDs e Lasers e o relativo baixo custo de operação desse ambiente quando comparado a outras técnicas de comunicação. Contudo, algumas dificuldades importantes são verificadas nessa abordagem. Inicialmente tem-se a dificuldade da obtenção de fontes de luz capazes de produzirem fótons únicos com confiabilidade aceitável, por motivos já discutidos anteriormente, e taxa de geração de fótons suficiente para as necessidades de desempenho verificadas pelos sistemas de telecomunicações modernos. Adicionalmente, se sabe que os estado de polarização de fótons transmitidos por fibra óptica são alterados ao longo do percurso, assim como sua intensidade, devido a atenuação intrínseca gerada pela fibra, o que produz erros no estabelecimento de protocolos de criptografia quânica. Isso exige que fibras ópticas para esse fim sejam extremamente eficientes, além da utilização de uma técnica capaz de recuperar a polarização inicial de um fóton.

Apesar das dificuldades na implementação de protocolos de criptografia quântica por fibra óptica, essa é a abordagem mais desenvolvida atualmente. O desenvolvimento de técnicas de tratamento das dificuldades relacionadas a manipulação de fótons polarizados em fibras, assim como a obtenção de fontes de luz mais precisas e confiáveis, permitiram que alguns dispositivos comerciais de distribuição de chaves quânticas fossem produzidos. Alguns exemplos são os dispositivos comercializados pelas empresas MagiQ e idQuantique, capazes de estabelecer uma chave criptográfica por meio do protocolo BB84 por distâncias de até 100km (na seção 7.2.2 são apresentadas e comparadas as informações sobre o desempenho nominal dos equipamentos fabricados por essas companhias).

Abordagens experimentais de implementação de criptografia quântica por fibra óp- 
tica são também responsáveis pelos melhores índices de desempenho na geração de fótons polarizados e pelas distâncias máximas alcançadas. A evolução que alguns do principais grupos de pesquisa têm alcançado na implementação de criptografia quântica por fibra óptica é bem ilustrada pela evolução dos resultados produzidos pelo NIST e implementados na DARPA Quantum Network nos últimos dois anos, sendo o índice de desempenho obtido pelo NIST o atual recorde de velocidade para implementação de protocolos quânticos em fibra óptica. Em 2006 o NIST obteve um canal de fibra óptica para distribuição de chaves quânticas operando a 4, 14 Mbits/s em um fibra com mais de $1 \mathrm{~km}$, mantendo um QBER menor que 3, 4\%. Ainda em 2006 o NIST estendeu o resultado anterior para fibras da ordem de centenas de quilômetros, estabelecendo o atual recorde de 148, $7 \mathrm{~km}$, utilizando um comprimento de onda tipicamente verificado na comunicação atual por fibras e um eficiente detector de fótons desenvolvido no instituto (HISKETT et al., 2006). Em 2007 o NIST, bateu seu próprio recorde anunciando a realização do protocolo $\mathrm{BB} 84$ a taxa de $\approx 2$ Gbits/s em uma fibra de mais de $1 \mathrm{~km}$, com perspectiva de extender a distância máxima obtida para centenas de quilômetros ainda este ano (MA et al., 2007). Adicionalmente, o NIST tem demonstrado o estabelecimento do protocolo BB84 para múltiplos usuários (TANG et al., 2006).

Os avanços apresentados anteriormente, dentre resultados de outras instituições não citados, indicam que a implementação do protocolo BB84 por fibra óptica vem alcançando índices suficientes para sua utilização em algumas aplicações práticas. Contudo, mesmo diante desses resultados, o cenário de uma rede quântica de cobertura global parece ainda muito distante quando considerado os milhares de quilômetros de algumas das fibras ópticas utilizadas nas modernas redes de telecomunicações mundiais. $\mathrm{Na}$ operação atual, repetidores de sinal são utilizados em intervalos de longas fibras para amplificar periodicamente o sinal e compensar a atenuação sofrida na transmissão. Contudo, como discutido, sinais quânticos são essencialmente distintos dos clássicos, sendo degradados quando ocorre qualquer interferência, o que é o caso da amplificação. Há, contudo, algumas propriedades ópticas que permitem que sejam obtidos alguns dispositivos capazes de realizar um procedimento equivalente a amplificação em sinais quânticos sem interferir nos estados do sistema. Uma dessas propostas é apresentada em (JACOBS; PITTMAN; FRANSON, 2002). Contudo, este é um campo ainda em desenvolvimento onde não existe nenhum resultado prático. Adicionalmente, espera-se que 
seja possível estabelecer comunicação com dispositivos móveis, o que seria impossível no caso da implementação por fibra. Assim, a existência de uma segunda abordagem, representada pela implementação de protocolos quânticos por espaços abertos, é fundamental para a aplicação da criptografia quântica em casos práticos.

\subsubsection{Criptografia quântica em comunicações por espaço aberto e satélites}

A implementação de protocolos de criptografia quântica por espaços abertos possui algumas vantagens e novos desafios quando comparada a abordagem por fibra óptica. Um dos principais benefício decorre do grande coeficiente de transmissão do ar para frequências de luz específicas, sendo a atmosfera fracamente dispersiva nesses casos e essencialmente isotrópica, mantendo com maior facilidade a polarização de fótons transmitidos por espaço aberto (ELBERT, 2002). Como principal desafio tem-se que devido ao fato da implementação de criptografia quântica requisitar a manutenção de um feixe contínuo de luz entre as partes da comunicação, a implementação por espaço aberto é estritamente direcional. Diferente daquilo que acontece com a comunicação por rádio frequência, a implementação de criptografia quântica exige a manutenção de uma visada direta entre os elementos da comunicação, com prejuízo de interrupção da comunicação na presença de um obstáculo físico. Adicionalmente, apesar de feixes de luz não sofrerem interação eletromagnética como acontece no caso das telecomunicações por rádio frequência, o que permite a transmissão eficiente destes em espaços onde há intensa propagação de rádio frequências, a detecção de feixes de luz sofre interferência da luminosidade verificada na atmosfera. Esse fato prejudica a operação de protocolos de criptografia quântica por espaço aberto em algumas situações práticas, como a operação em regiões metropolitanas em períodos noturnos.

Apesar das dificuldades na operação de protocolos quânticos por espaço aberto, a possibilidade de obtenção de uma rede de troca de chaves quânticas por meio desta abordagem se mostra mais viável, tornando a implementação de criptografia quântica por espaços abertos o foco de diversas pesquisas que apresentaram avanços relevantes nos últimos anos.

Os melhores resultados alcançados na implementação desta abordagem decorrem 
dos esforços de desenvolvimento do projeto SECOQC, representado pela Universidade de Munique, em conjunto com a companhia QinetiQ. O projeto SECOQC é responsável por uma abordagem bastante eficiente, que detém o recorde de maior distância de implementação do protocolo BB84 por espaço aberto e que pode tornar possível a obtenção de uma rede global de chaves quânticas. O projeto é responsável pela demonstração experimental da execução do protocolo BB84 por uma distância de 144 km entre duas ilhas no atlântico a uma taxa de transmissão da ordem de KBits/s, mantendo um QBER de $\approx 5 \%$ (SCHMITT-MANDERBACH et al., 2007). Adicionalmente, no mesmo trabalho é demonstrado teoricamente a viabilidade do estabelecimento do protocolo BB84 operando por espaço aberto a distâncias acima de $1600 \mathrm{~km}$ mantendo-se o índice de desempenho obtido na realização experimental. Considerando que a tecnologia desenvolvida nesta abordagem na construção de dispositivos de geração e detecção de fótons permite a obtenção destes com massa suficientemente pequena para serem embarcados em satélites, na referência (SCHMITT-MANDERBACH et al., 2007) é apontada a viabilidade de um cenário em que utilizando satélites de baixa órbita (LEO-Satellites), satélites de altitude média e satélites geoestacionários (GEO-Satellites) seja possível estabelecer uma rede de distribuição de chaves quânticas de escala global. A análise de viabilidade técnica da construção de uma rede global de distribuição de chaves quânticas utilizando a tecnologia de satélites atual foi demonstrada conceitualmente em (POPPE et al., 2004) utilizando um arranjo semelhante ao mostrado na figura 19. A utilização de criptografia quântica por meio de uma malha de satélites e o tratamento de elementos móveis é demonstrada em detalhes nas referências (POPPE et al., 2004; KURTSIEFER et al., 2002; RIGUIDEL, 2004).

Os próximos capítulos exploram em maiores detalhes os resultados obtidos pelo projeto SECOQC em conjunto com a companhia QinetiQ e desenvolvem um cenário em que considerando estes resultados seja possível utilizar o protocolo BB84 como mecanismo de segurança de uma operação prática.

\subsection{Considerações finais}

Os fundamentos conceituais apresentados anteriormente e os desenvolvimentos técnicos discutidos permitem considerar a utilização da criptografia quântica como meca- 


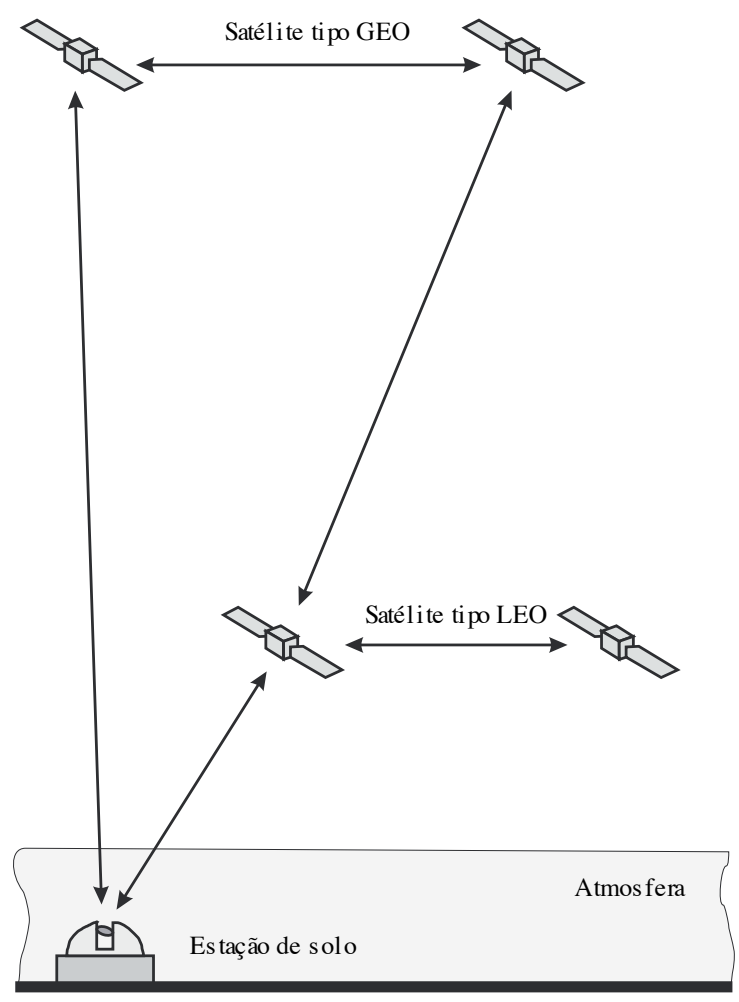

Fig. 19: Estrutura para distribuição de chaves quânticas utilizando satélites

nismo de segurança em cenários práticos, considerando que tecnicamente é possível obter uma rede de troca de chaves quânticas em escala global. A possibilidade da comunicação por espaço aberto e entre elementos móveis, como demonstrada em (POPPE et al., 2004), possibilita novas perspectivas na utilização de protocolos de criptografia quântica.

Entretanto, a despeito dos avanços já obtidos, muitos aspectos necessários a uma utilização prática de protocolos quânticos nas telecomunicações atuais ainda não foram tratados. Um exemplo consiste no fato que não há ainda medidas ou estimativas de índices de confiabilidade e disponibilidade para equipamentos que implementam protocolos quânticos possuiriam em um cenário realista de operação, aspecto fundamental em muitas redes de telecomunicações. Considerando, entretanto, os atuais índices de desempenho e distância máxima obtidos e considerando ainda que estes podem ser alcançados em um cenário com confiabilidade e disponibilidade suficientes para os padrões médios exigidos pelas principais redes de telecomunicações, é possível propor cenários de integração de protocolos quânticos em infra-estruturas de telecomunicações já existentes e avaliar se a tecnologia disponível atualmente permite a implementação prática dessa abordagem. 
Nos capítulos seguintes é feita uma discussão do impacto que a segurança da informação tem sobre algumas redes de telecomunicações, a apresentação de um caso de estudo, a proposição de cenários de utilização do protocolo BB84 como mecanismo de segurança nessa infra-estrutura e uma avaliação dos índices de desempenho esperados para a implementação prática deste cenário. 


\section{$5 \quad$ Redes de missão crítica: a ATN}

Há um conjunto de redes de telecomunicações atuais que exigem o atendimento a alguns requisitos mínimos de operação, como desempenho, disponibilidade, confiabilidade, segurança, dentre outros, sendo que o não atendimento destes está associado a perdas materiais, financeiras, patrimoniais ou mesmo humanas. Essas redes são frequentemente referenciadas como redes de missão crítica.

Um exemplo de rede de telecomunicações que é altamente dependente do atendimento de requisitos de operação é representado pelas telecomunicações aeronáuticas. Esta rede passa por um período de transição associado a migração progressiva de canais de comunicação baseado em voz para a utilização de canais de dados digitais, o que gera novos requisitos na sua operação. A comunicação aeronáutica atualmente utiliza canais de dados digitais como suplemento para comunicação por voz e como meio para permitir diversas melhorias nos serviços de tráfego aéreo. Como forma de definir e regulamentar o desenvolvimento do futuro da comunicação aeronáutica, a Organização da Aviação Civil Internacional (ICAO, na sigla em inglês para International Civil Aviation Organization), publicou em 1991 o conceito da Aeronautical Telecommunication Network (ATN) como sendo uma rede que tal como a Internet seria baseada na arquitetura de comunicação OSI (Open Systems Interconnection) e que suportaria a comunicação global e todo os canais digitais previstos nos manuais da ICAO (ICAO, 2002). Contudo, a utilização de canais digitais nas telecomunicações aeronáuticas adiciona um novo requisito em sua operação: a manutenção da segurança da informação.

Neste capítulo é mostrado como a atual especificação proposta para a ATN trata o requisito de manutenção de segurança da informação e como este pode se tornar vulnerável diante da presença de novas formas de processamento de informação, como a computação quântica. Para isso, na seção 5.1 é apresentada um introdução resumida da 
especificação proposta para a ATN e os principais serviços a serem oferecidos por esta. A seção 5.2 mostra como mecanismos de manutenção de segurança são obtidos na atual especificação da ATN. Por fim, nas considerações finais do capítulo é apresentada uma discussão sobre como as técnicas de segurança propostas para a ATN podem estar sob risco em um período próximo e os impactos deste fato na segurança física da operação aérea.

\subsection{Visão geral da ATN}

A ATN é uma rede de dados que tem como como características (RIGUIDEL, 2004):

(i) provimento de um serviço de comunicação comum para todos os Serviços de Comunicação de Tráfego Aéreo (ATSC, Air Traffic Services Communication) e Serviço de Comunicação para Indústria Aeronáutica (Aeronautical Industry Service Communication, AINSC). As comunicações podem ser tanto de elementos em solo para elementos em solo (solo/solo) ou elementos no ar para elementos em solo (ar/solo);

(ii) integração e utilização de redes de comunicação e infra-estrutura de redes já disponíveis quando possível. Desta forma, preservando os investimentos realizados em redes como CIDIN e X25;

(iii) atendimento de requisitos de segurança para as aplicações de ATSC e AINSC e acomodar os diferentes níveis de serviços requisitados por cada aplicação ATSC e AINSC;

(iv) fornecimento de um serviço de comunicação robusto e confiável aos usuários. $\mathrm{O}$ desenvolvimento da ATN deve assegurar uma alta disponibilidade, evitando pontos simples de falha e permitindo múltiplos caminhos para um mesmo destinatário com mudança de alternativa dinâmica tanto para comunicação entre pontos fixos e móveis;

(v) suporte a sistemas móveis, tal como aeronaves em trânsito, em uma grande variedade de redes de comunicação móveis como AMSS, VDL e Mode S. Deve 
ser possível a qualquer sistema se comunicar com qualquer equipamento de uma aeronave em qualquer parte do mundo.

Os serviços que são providos pela ATN são implementados de acordo com o Serviço de Transporte OSI (OSI Transport Service), do padrão ISO 8072. As aplicações da ATN são construídas de acordo com a arquitetura conhecida como Arquitetura de Camada Superior (ULA, Upper Layer Archicteture) baseada nas camadas do Modelo de Referencia OSI (OSI Reference Model). Existem sete camadas, onde dois tipos de entidades são identificadas:

(i) Sistemas finais, tal como computadores, usando as 7 camadas;

(ii) Sistemas intermediários, tal como roteadores, utilizando as 3 camadas inferiores.

As sete camadas OSI são listadas abaixo, da superior a inferior:

(i) Camada de aplicação: semântica de troca da informação dos pontos extremos;

(ii) Camada de apresentação: sintaxe de troca informação dos pontos extremos;

(iii) Camada de sessão: formato de troca da informação dos pontos extremos;

(iv) Camada de transporte: fluxo de controle da troca de informação dos pontos extremos;

(v) Camada de rede: estabelecimento, manutenção e finalização da conexão;

(vi) Camada de dados: sincronização e controle de erros sobre canal físico;

(vii) Camada física: gerenciamento do canal físico.

As três camadas superiores estão frequentemente relacionadas a funções que são utilizadas para o estabelecimento e distribuição da conexão e para a codificação de informação, sendo a operação de mecanismos de segurança da informação realizados nestas. 


\subsubsection{Aplicações da ATN no CNS/ATM}

As principais e também as primeiras aplicações especificadas para a primeira fase de implementação da ATN consistem nas aplicações de Comunicação, Navegação e Vigilância (CNS, Communications, Navigation and Surveillance) e Gerenciamento de Tráfego Aéreo (ATM, Air Traffic Management). Os serviços disponibilizados pelas aplicações CNS/ATM são descritos resumidamente abaixo.

(i) Gerenciamento de contexto (Context Management - CM), provimento de uma meio de busca por serviços de comunicação dentro de uma região de vôo, e para um sistema de solo ou controlador direto de uma aplicação CM de uma aeronave, um meio de entrar em contato com uma região de vôo diferente;

(ii) Vigilância Automática Dependente (Automatic Dependent Surveillance - ADS), desenvolvida para gerar relatórios automáticos de uma aeronave em vôo para sistemas em solo. Essa informação deve ser fornecida sob demanda e sob emergência. Dados como posicionamento e trajetória de uma aeronave e dados meteorológicos são informações típicas deste serviço;

(iii) Canal de Comunicação de Dados Controlador-Piloto (Controller-Pilot Data Link Communications - CPDLC), provimento de uma comunicação orientada a uma mensagem de duas vias incluindo um conjunto de mensagens de esclarecimentos, informação ou requisição correspondendo a fraseologia corrente empregada nos procedimentos de controle de tráfego aéreo (Air Traffic Control);

(iv) Serviço de Informação de Vôo (Flight Information Service - FIS), dar suporte a uma variedade de serviços de informação, provendo informação sobre o solo e uma aeronave. Incluindo informações sobre condições de aeroportos e condições climáticas.

(v) Facilidade de Intercomunicação de Dados ATS (Interfacility Data Communication - AIDC) provimento de um meio para a troca de informações de controle de tráfego aéreo (ATC) entre Unidades de Servico de Tráfego Aéreo que fazem o suporte de funçoes ATC, incluindo notificações de aproximação de vôos dos limites 
de uma Região de Informação de Vôo (Flight Information Region), coordenação das condições de intersecção de regiões, e transferência de controle.

(vi) Sistema de Manuseio de Mensagens Aeronáuticas (Aeronautical Message Handling System - AHMS) provimento de uma meio para troca e distribuição de mensagens de tráfego entre Unidades de Serviço de Tráfego. Este serviço pode ser utilizado para provimento de serviços de mensagem, incluindo correio eletrônico e troca eletrônica de dados.

\subsection{Implementação de segurança da informação na ATN}

Em um estudo elaborado pelo European Organisation for the Safety of Air Navigation (EUROCONTROL) é apontado que o tráfego de informação digital na ATN a torna significativamente vulnerável, necessitando da implementação de mecanismos de segurança da informação (MCMATH, 2003). As principais ameaças identificadas são:

(i) Ameaça a mensagens de controle de tráfego aéreo, tanto no sentido ar/solo como solo/solo:

- Modificação;

- Repetição;

- Mascaramento;

- Atolamento (jamming).

(ii) Ameaças ao Sistema de Manuseio de Mensagens (MHS):

- Modificação;

- Mascaramento.

(iii) Ameaças ao gerenciamento do sistema OSI:

- Modificação;

- Repetição; 
- Mascaramento;

- Modificação não autorizada da base de informação de gerenciamento.

(iv) Para todas as aplicações existe a vulnerabilidade de ataques conhecidos como negação de serviço (Denial of Service), que tem impacto sobre as mensagens de controle de tráfego da forma:

- Atolamento de canais ar/solo;

- Sobrecarregamento da ATN com pacotes de dados;

- Provacamento de interrupções e falha no canal de dados;

- Alteração não autorizada de rota da informação.

A conclusão apontada por esse levantamento consiste na necessidade da proteção por meio de assinatura digital (seção 2.4), provendo tanto a autenticação do remetente quanto uma verificação de integridade de alta qualidade. Adicionalmente, em muitos caso é altamente desejável a disponibilidade de um mecanismo eficiente de encriptação de mensagens. Dessa forma, os requisitos primordiais de segurança da informação para a ATN podem ser resumido por:

(i) Autenticação da fonte de mensagens:

- proteção contra retração de relatório;

- proteção contra mascaramento de controlador.

(ii) Verificação de integridade de mensagens:

- proteção contra substituição de mensagem;

- proteção contra repetição de mensagem.

(iii) Manutenção da confidencialidade:

- proteção contra acesso indevido a mensagens de controle. 


\subsubsection{Mecanismo de gerenciamento de chaves criptográficas}

Os requisitos de segurança da informação apontados na seção anterior levaram a escolha da utilização de algoritmos assimétricos de criptografia como mecanismo de segurança da informação da ATN, de forma a satisfazer os requisitos (ICAO, 2002):

(i) A ATN necessita de um mecanismo apropriado para a instalação e atualização segura de chaves privadas no contexto da segurança dos canais de comunicação de dados;

(ii) O mecanismo de segurança da ATN requer o suporte de uma Infra-estrutura de Chave Pública (ICP);

(iii) É necessário a instalação e atualização segura de chaves privadas para os elementos da aviônica.

Uma ICP é um sistema gerenciador de chaves composto por uma entidade confiável conhecida como Autoridade Certificadora (Certificate Authority, CA). Uma Autoridade Certificadora gera e distribui chaves públicas autênticas, confiáveis, normalizadas como certificados X.509, relacionando uma identidade com uma chave pública. É responsabilidade de uma CA a emissão da Lista de Revogação de Certificados (Certificates Revocation Lists - CRL), quando um certificado é revogado. Considerando que, por medida de segurança, chaves públicas devam ser renovadas com certa frequência, a ação de revogação de certificados corresponde a emissão de uma lista por parte da CA indicando quais as chaves públicas não são mais válidas. Isso impede que chaves que não estão mais em uso sejam inadequadamente utilizadas. Não há uma definição de padrão para ICP, sendo que aquelas existentes frequentemente não são interoperáveis.

De acordo com a especificação da ICAO (ICAO, 2002), deve existir uma Autoridade Certificadora de Estado, i.e., uma CA assinada pelo estado (por exemplo, os EUA assinam a Federal Aviation Administration - FAA - ou a União Européia assina o Eurocontrol), sendo que estas CA estabelecem relação de confiança com outras entidades. As Autoridades Certificadoras são responsáveis pela certificação das entidades da ATN. Além disso, existe uma Agência de Operação de Autoridades Certificadoras (Operating Agency Certificate Authority, OACA), correspondente às empresas aéreas por exemplo, 
que são subordinadas a Autoridades Certificadoras e certifica aeronaves dentro de seus domínios. A verificação de uma dada certificação é feita por meio da verificação da chave pública correspondente a OACA. Uma vez feito isso, pode-se verificar o certificado da OACA com sua chave pública correspondente de sua CA.

Uma ICP utiliza métodos de criptografia de chave pública, que como discutido no capítulo 3 são fundamentados em problemas matemáticos de difícil solução computacional. Uma preocupação iminente que decorre desse fato consiste na fragilidade com que o sistema de distribuição de chave pública da ATN pode estar submetido. Considerando que o segredo e validade de um certificado depende de toda a segurança da Infra-estrutura de Chaves Públicas, se a chave privada de uma CA ou OACA for violada, toda a ICP poderá ser corrompida também. A sessão seguinte mostra como a segurança da ATN na especificação atual depende de uma ICP confiável.

\subsubsection{Segurança de comunicações na ATN utilizando uma ICP}

A figura 20 resume a estrutura de roteamento entre as entidades da ATN. De acordo com essa estrutura, as aplicações da ATN podem ser divididas em duas categorias principais: aplicações ar/solo (A/S) e aplicações solo/solo (S/S).

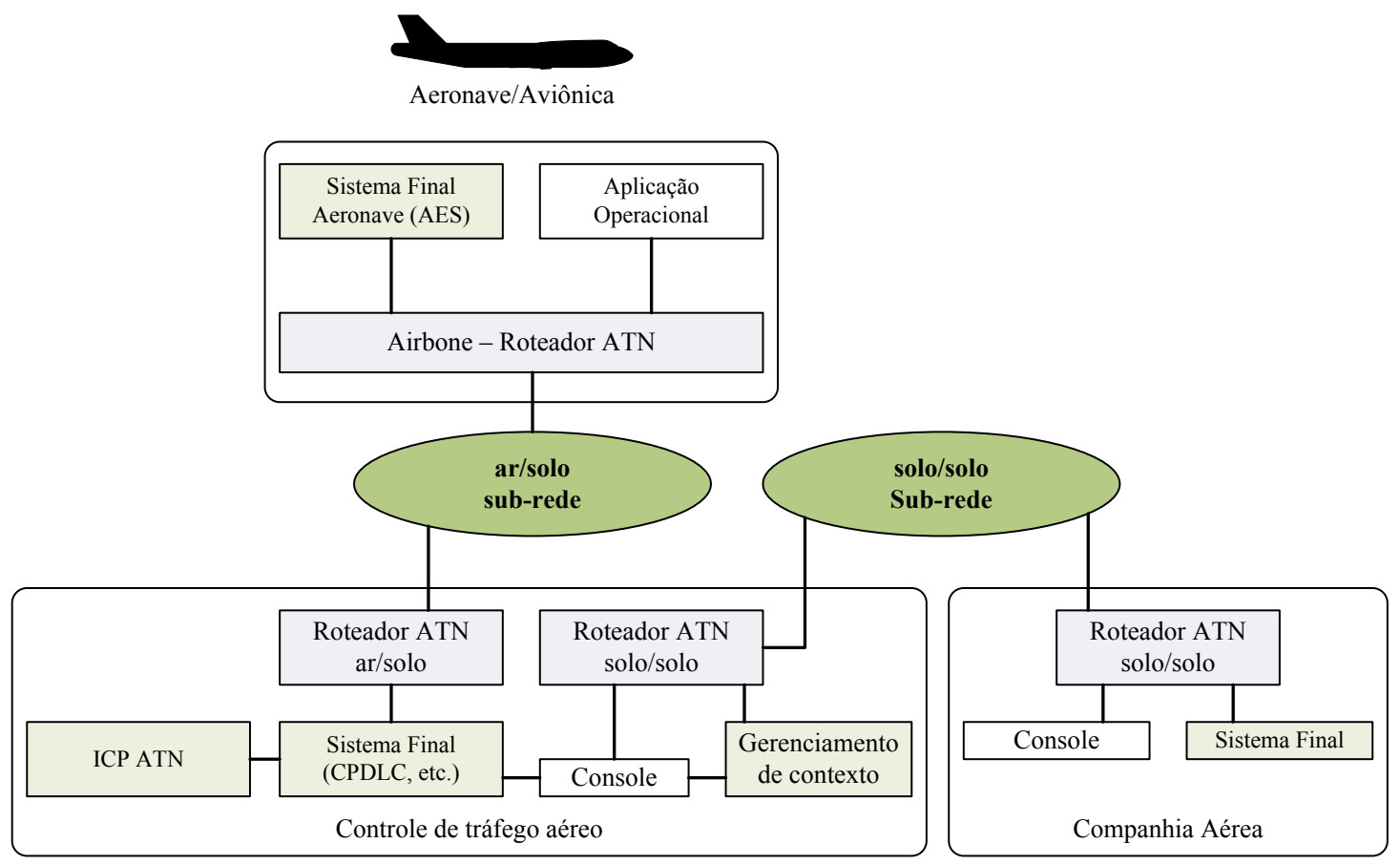

Fig. 20: Estrutura de roteamento da ATN 
Uma das aplicações A/S relevantes para a segurança de comunições na ATN é conhecida como Aplicação de Gerenciamento de Contexto (Context Management Application, CMA). Esta aplicação fornece um mecanismo para o Sistema Final da Aeronave (Airbone End System, AES) se registrar na rede ATN no intuito de se comunicar e utilizar aplicações A/S requeridas ou fornecidos pelo AES.

A implementação de segurança para aplicações $\mathrm{S} / \mathrm{S}$ empregam soluções similares àquelas encontradas nas comunicações via cabeamento. Como é discutido em detalhes na seção 5.3, as aplicações do tipo A/S, devido a características de seus canais de dados, introduzem novas ameaças na operação segura de uma aeronave.

Os requisitos de segurança apresentados na seção 5.2.1 estabelecem como prioridade o tratamento de ameaças relacionadas a autenticação dos canais de dados. Contudo, a utilização de uma arquitetura baseada em uma ICP, tal como foi proposta, permite adicionalmente o serviço de proteção do conteúdo da mensagem trafegada (este serviço decorre trivialmente da manutenção de uma ICP, como foi discutido na seção 2.3). Essa característica adicional se mostrará bastante útil em diversos casos, quando por exemplo, a informação de controle trafegada tem caráter estratégico.

A comunicação entre um AES e uma aplicação A/S em uma Estação de Solo (Ground Station, GS) ocorrerá mediante a execução de um cenário básico apresentado a seguir. Uma aplicação típica deste gênero seria a aplicação de Canal de Comunicação Controlador-Piloto (Controller-Pillot Data Link Communications, CPDLC).

Passo 1 Um AES cria uma Requisição de Entrada de Registro de CPDLC no CM e envia esta ao CMA;

Passo 2 O CMA envia uma Resposta de Registro de Entrada de CPDLC de volta ao AES;

Passo 3 O AES e a aplicação CPDLC estabelecem uma chave de sessão secreta;

Passo 4 O AES e a aplicação CPDLC protegem sua troca de mensagens por meio da utilização da chave de sessão secreta.

No cenário proposto, o AES deve manter duas chaves de sessão secreta, uma chave de sessão para o CMA e uma outra para o CPDLC. A primeira delas mantém a comu- 
nicação segura com a aplicação de Gerenciamento de Contexto (CM) e a outra é usada para manter segura a comunicação com a aplicação CPDLC.

Atualmente, o gerenciamento de chaves de sessão para as diversas aplicações é realizado pela ICP da ATN, que utiliza os seguintes esquemas criptográficos (ICAO, 2002):

(i) Esquema de encriptação: encriptação assimétrica ou simétrica;

(ii) Esquema de assinatura digital: encriptação assimétrica e função de Hash;

(iii) Esquema de código autenticador de mensagem: função de Hash.

\subsection{Considerações finais do capítulo}

Tradicionalmente a análise de segurança - utilizando aqui o sentido expresso pelo termo internacionalmente reconhecido como safety - está associada ao tratamento de estados que levem a acidentes onde ocorra perdas de ordem humana ou material. Dessa forma, a análise de segurança está mais envolvida com a mitigação dos riscos associados à falhas no âmbito de desenvolvimento e implementação de um projeto. Em contrapartida, a segurança - utilizando aqui o sentido do termo reconhecido como security - esta tradicionalmente relacionada ao tratamento de aspectos de acesso a informação, tendo como objetivo principal mitigar os casos em que há risco de acesso indevido dentre outros aspectos. A atual tendência de maior utilização de comunição digital entre aeronaves e estações de solo e aeronaves entre si, com consequente migração para ATN, constitui um exemplo nítido de sistema em que os dois conceitos estão fortemente relacionados. Nesse caso, falhas no âmbito da informação, representadas aqui por acesso indevido, corrompimento de informação e outras vulnerabilidades discutidas ao longo deste capítulo, podem levar a acidentes. Com isso, os requisitos de safety do sistema devem considerar requisitos de security na implementação de um rede unificada de comunicação aeronáutica.

A forma atual de se alcançar os requisitos de security em telecomunicações aeronáuticas, evitando estados de falha de safety, é a utilização de algoritmos criptográficos clássicos, como discutido nas seções anteriores. Especialmente, a utilização de algorit- 
mos criptográficos assimétricos é fundamental para a implementação da Rede de Telecomunicações Aeronáuticas, ATN, como proposta nas referências discutidas na seção 5.2.2.

Uma questão, entretanto, que se torna extremamente relevante são os resultados apresentados em detalhes no capítulo 3, que apontam para o enfraquecimento dos métodos de criptografia atuais em um cenário de disponibilidade de computação quântica. Em contrapartida, a manutenção da segurança do canal de comunicação no ambiente aeronáutico se torna cada vez mais crítica a medida que mais funções de uma aeronave passam a ter dependência de informação digital.

Os resultados obtidos na implementação de protocolos de criptografia quântica apresentados na seção 4.2 permitem considerar a utilização destes como um mecanismo alternativo para se estabelecer comunicação segura entre elementos da ATN. Adicionalmente, esta possibilidade apresenta algumas novas propriedades bastante úteis em redes aeronáuticas. A característica de detecção de tentativa de espionagem proporcionada pelos protocolos criptográficos quânticos, decorrente do teorema da não clonagem, permitem, ao contrário do que ocorre frequentemente na transmissão clássica de informação, na qual um sinal pode ser interceptado sem detecção, a obtenção de novos meios de tratar as ameaças à segurança da ATN levantadas anteriormente.

No capítulo 6 é apresentada uma proposta de cenários de como as técnicas desenvolvidas na seção 4.1 podem ser utilizadas como mecanismo de segurança no tráfego de informação de redes aeronáuticas, discutindo as principais dificuldades que podem ser encontradas e os desenvolvimentos necessários. 


\section{Estudo de caso: criptografia quântica em redes de telecomunicação aeronáuticas}

Qualquer tentativa de melhorar os mecanismos de manutenção da segurança da informação em telecomunicações aeronáuticas deve ser feito no contexto da construção da Rede de Telecomunicações Aeronáuticas (ATN), isto é, qualquer solução deve ser totalmente compatível com as especificações da ATN e deve necessariamente ser viável do ponto de vista de implementação prática.

Os resultados apresentados na seção 4.1 demonstram a possibilidade da utilização de métodos de distribuição de chaves quânticas (DCQ) no cenário aeronáutico. Este capítulo tem como objetivo apresentar o desenvolvimento de uma Infra Estrutura de Distribuição de Chaves Quânticas (IDCQ) como um mecanismo de troca de chaves de sessão entre dois pontos finais operando na estrutura atual da ATN. Para isso é discutido na seção 6.1 dois conceitos fundamentais para a operação prática de distribuição de chaves quânticas. Na seção 6.2 é apresentado como adequar a IDCQ às aplicações A/S e na seção 6.3 às aplicações S/S. Por fim, a seção 6.4 discute a utilização de uma IDCQ na rede ATN utilizando uma rede global de DCQ.

\subsection{Repetidores quânticos e encaminhadores de dados DCQ}

Tal como ocorre com sinais clássicos, seria esperado de um repetidor quântico que este recebesse um sinal quântico e redirecionasse este sem manipular diretamente os estados quânticos, i.e., sem realizar uma medida sobre estes ou ler seus conteúdos gerando 
assim a degradação dos estados quânticos. Como discutido anteriormente, existem algumas propriedades ópticas que permitem que tais dispositivos sejam obtidos (JACOBS; PITTMAN; FRANSON, 2002). Contudo, este campo ainda se encontra em desenvolvimento, não existindo nenhum resultado prático.

Há um segundo conceito de bastante utilidade representado pelo encaminhador de dados DCQ ou roteador DCQ. Este permite a obtenção de uma cadeia de elementos que estabelecem comunicação segura entre si utilizando protocolos de distribuição de chaves quânticas. Um encaminhador de dados DCQ deve possuir as seguintes características:

(i) Um encaminhador $k$ estabelece um canal de comunicação encriptado com o encaminhador $k-1$ baseada em uma chave secreta trocada utilizando um método de DCQ;

(ii) $\mathrm{O}$ encaminhador $k$ recebe dados encriptados do encaminhador $k-1$;

(iii) As informações são desencriptadas e armazendas na memória do encaminhador $k$;

(iv) $\mathrm{O}$ encaminhador $k$ estabelece um canal encriptado de comunicação de rádio com o encaminhador $k+1$ baseada na chave obtida pelo método DCQ;

(v) Os dados armazenados em $k$ são codificados e enviados ao encaminhador $k+1$.

Com foco na obtenção de uma solução viável será considerada a utilização dos encaminhadores de dados DCQ na obtenção do mecanismo de IDCQ da ATN. Apesar do repetidor quântico ser um dispositivo mais transparente no sentido de, em princípio, possuir um atraso menor por não necessitar de armazenar dados em um memória, este não será considerado neste trabalho devido a diversas questões práticas de desenvolvimento ainda não totalmente definidas na sua construção. $O$ fato de um armazenamento em memória ser necessário em um encaminhador de dados DCQ pode levantar a questão sobre a segurança da informação enquanto esta estiver armazenada na memória do dispositivo. Esta questão deixa de ser relevante quando se considera alguns fatores, como o fato que os encaminhadores de dados quânticos serão dispositivos essencialmente remotos (como será visto adiante, estes estarão embarcados em satélites) e que a comunicação com estes é feita de forma remota e mantida em segurança por um protocolo de 
criptografia quântica. Adicionalmente, a possibilidade de perda de um destes dispositivos e o possível extravio da informação armazenada em sua memória não constitui um risco uma vez que a informação de uma chave quântica somente é útil no momento da execução do protocolo.

A utilização dos encaminhadores de dados DCQ é possível e demonstrada em (KURTSIEFER et al., 2002), que utiliza as técnicas DCQ já descritas anteriormente. As provas de segurança apresentadas nas seções 4.1.5 e 4.1.6 garantem que um encaminhador de dados DCQ operando como um remente/destinatário confiável não apresenta nenhuma vulnerabilidade de segurança adicional.

Com isso, as seções seguintes demonstram como utilizar uma IDCQ para as duas principais classes de aplicações da ATN, considerando em um primeiro momento o funcionamento ideal do aparato físico utilizado. A inserção das condições de operação de um ambiente realista serão abordadas no capítulo 7 .

\subsection{IDCQ para aplicações A/S}

O mecanismo IDCQ é responsável pelo fornecimento confidencial de chaves de sessão compartilhadas entre dois pontos finais. Em aplicações A/S da ATN os pontos finais são sempre uma aeronave (AES) e uma Estação de Solo (GS) conectada a rede ATN.

Como as aeronaves encotram-se em grande parte do tempo em vôo, canais quânticos por espaço aberto são utilizados para aplicações A/S. A tecnologia DCQ apresenta, como discutido na seção 4.1.1, características bastante específicas referentes aos pontos de comunicação quando comparada aos métodos clássicos de distribuição de chaves criptográficas. Assim, a utilização de um mecanismo IDCQ para operação de aplicações A/S da ATN deve considerar alguns cenários específicos referentes a arquitetura do mecanismo IDCQ, ou seja, o arranjo do transmissor e receptor. As seções seguintes discutem as três possibilidades de construção de uma IDCQ operando na rede ATN. 


\subsubsection{IDCQ baseada em fonte de fótons no solo}

Neste cenário a fonte de fótons é mantida em uma estação de solo. Um feixe de laser é utilizado na realização do protocolo BB84 (ver seção 4.2.2) como canal de subida (up-link) para um receptor embarcado em uma aeronave (AES), permitindo assim a negociação de uma chave de sessão como mostrado na figura 21.

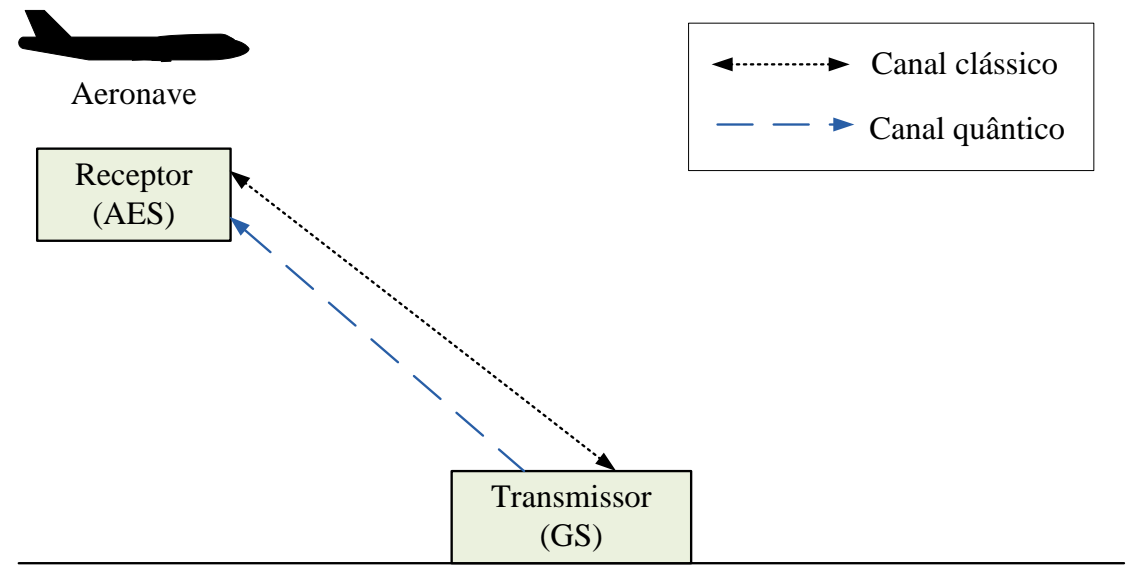

Fig. 21: IDCQ baseada em solo

A abrangência de grandes áreas como discutido na seção 4.2.2 pode ser feita por meio de satélites com equipamento embarcado agindo como um encaminhador de dados DCQ, como mostrado na figura 22

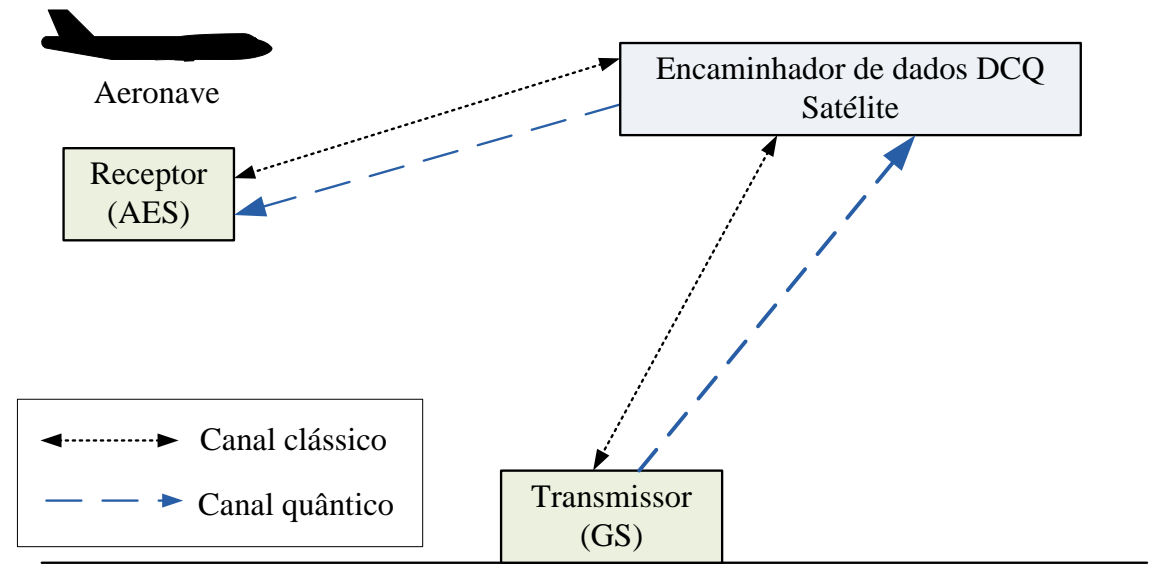

Fig. 22: IDCQ baseada em solo utilizando encaminhador DCQ

\subsubsection{IDCQ baseada em fonte de fótons embarcada em aeronave}

Um segundo cenário corresponde a embarcar o equipamento de transmissão de fótons na aeronave. Desse forma, utilizando um feixe de laser realizando o protocolo 
BB84, orientado para um receptor em uma Estação de Solo, obtém-se um canal de descida (down-link) que permite a negociação de uma chave de sessão, como mostrado na figura 23

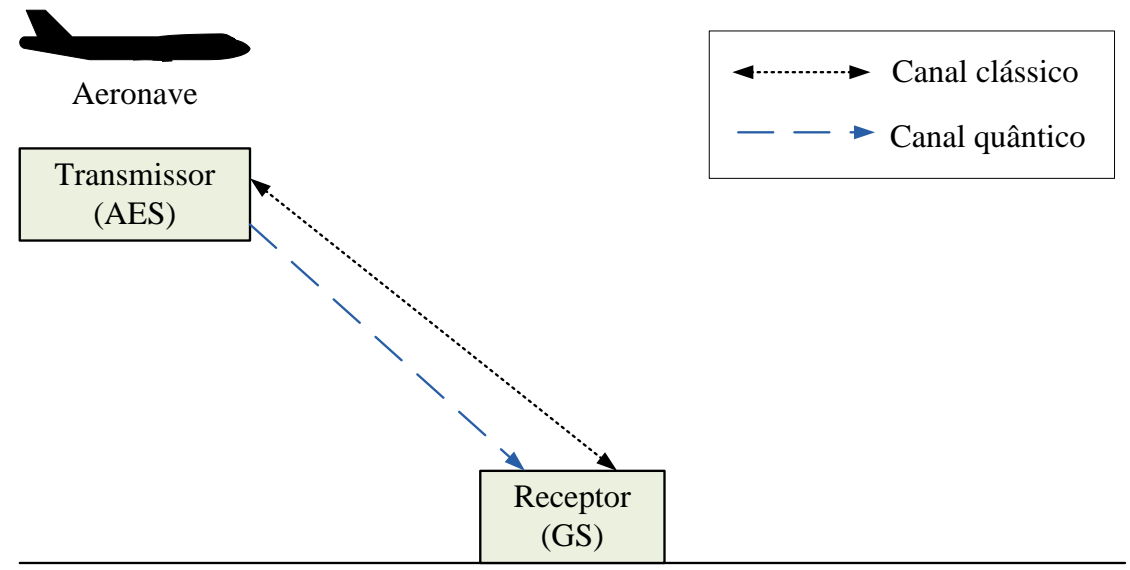

Fig. 23: IDCQ baseada em aeronave

Tal como no caso anterior de uma IDCQ baseada em fonte de fótons no solo, é possível também neste cenário utilizar satélites com equipamento embarcado agindo como um encaminhador de dados DCQ para cobrir amplas áreas, como mostrado na figura 24 .

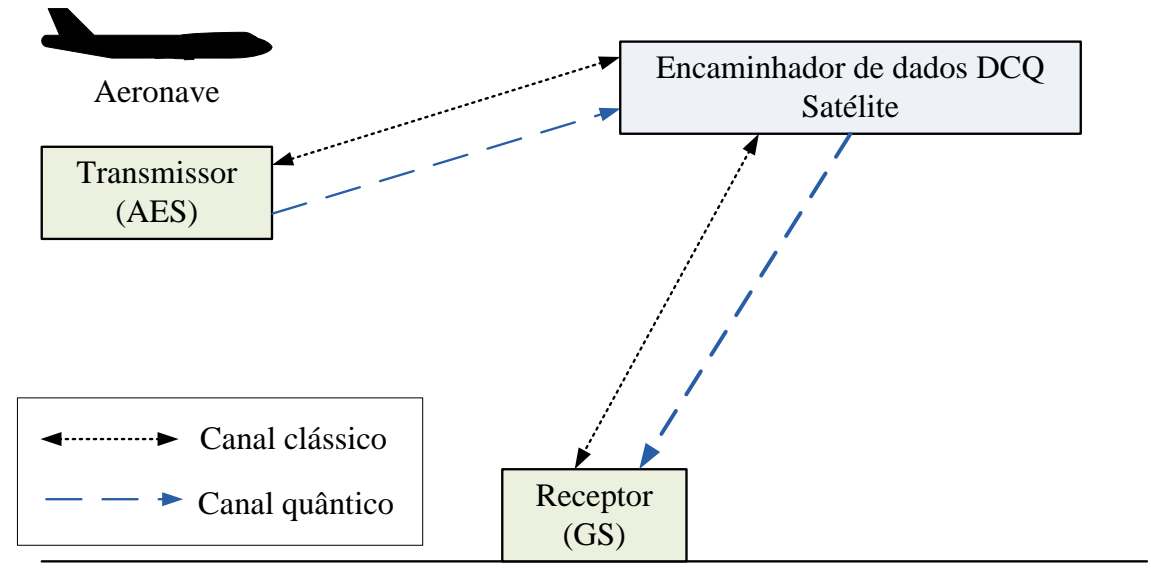

Fig. 24: IDCQ baseada em aeronave utilizando encaminhador DCQ

\subsubsection{IDCQ baseada em fonte de fótons embarcada em satélite}

Em um terceiro cenário a fonte de transmissão de fótons é embarcada em um satélite. Esta solução é mais complexa devido ao fato da negociação da chave de sessão 
não ser feita diretamente entre a aeronave (AES) e a Estação de Solo (GS). O estabelecimento de comunicação acontece por meio dos seguintes procedimentos:

(i) Um satélite estabelece uma chave secreta $K 1$ com uma estação de solo utilizando um mecanismo DCQ. Utilizando a chave $K 1$ como chave de sessão, o satélite estabelece comunicação segura com a ATN por meio de uma canal de comunicação por rádio COM1.

(ii) O satélite anterior estabelece então uma nova chave secreta $K 2$ com uma aeronave. Usando $K 2$ como chave de sessão estabelece comunicação segura por meio de um canal de comunicação por rádio COM2.

(iii) Por fim, é possível a negociação de uma chave secreta utilizando os canais de comunicação seguros COM1 e COM2 para estabelecer comunicação entre a ATN e a aeronave (AES).

Este cenários é ilustrado na figura 25, sendo possível ainda a utilização de outros satélites agindo como encaminhadores de dados DCQ como aquele mostrado na figura 26.

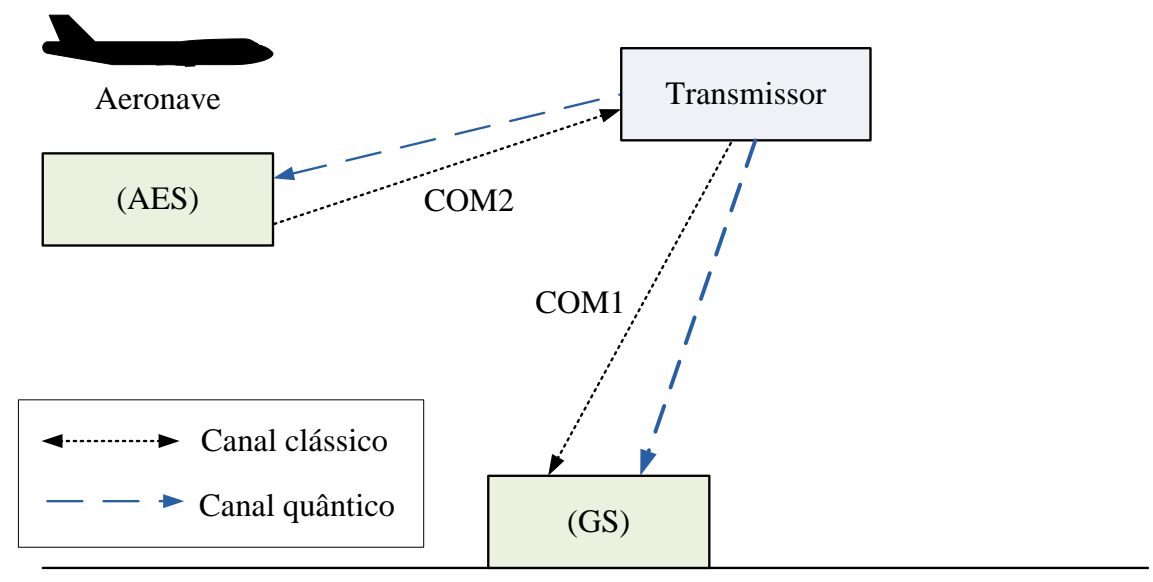

Fig. 25: IDCQ baseada em satélites

O cenário anterior tem sido apontado como o melhor cenário de realização de protocolos de criptografia quântica por espaços abertos e utilizando satélites (SCHMITTMANDERBACH et al., 2007). Isso se deve principalmente ao fato que os efeitos de interferência que podem ser causados pela atmosfera são menores quando o sinal é gerado 


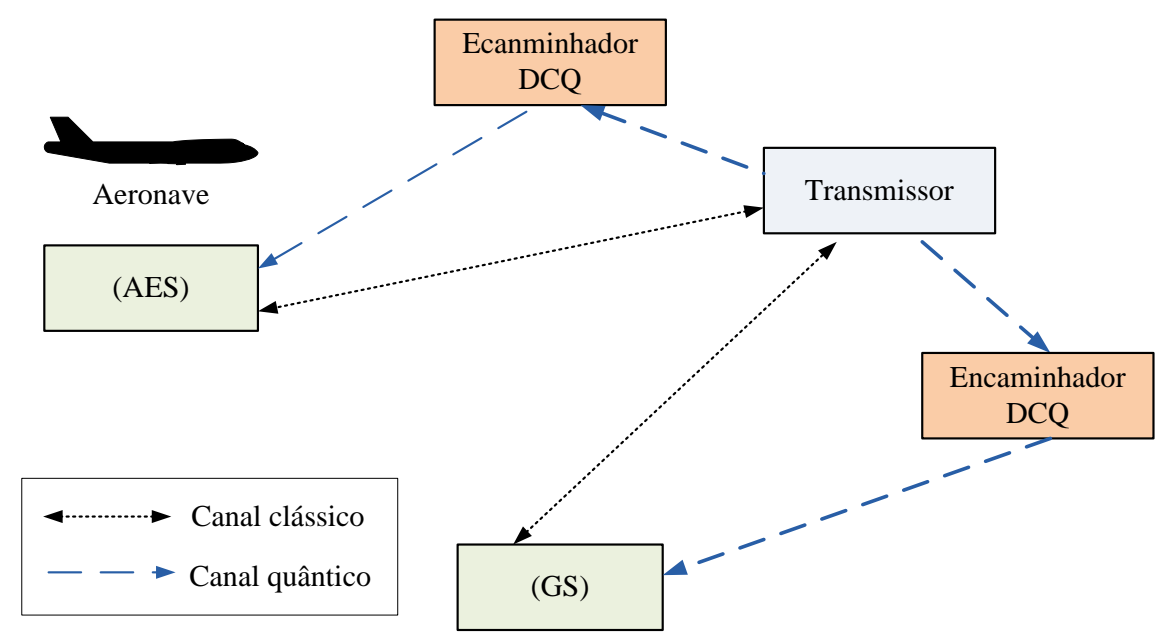

Fig. 26: IDCQ baseada em satélites utilizando encaminhadores DCQ

a partir do satélite (SCHMITT-MANDERBACH et al., 2007). Assim, este será o cenário considerado nesta pesquisa para a implementação de uma IDCQ na ATN.

A chave de sessão estabelecida entre os pontos deste cenário permite a obtenção dos canais clássicos seguros COM1 e COM2 por meio da utilização da Cifra de Vernam como sistema de encriptação, como já discutido anteriormente. A implementação da Cifra de Vernam pode ser feita facilmente em qualquer canal de comunicação clássico, incluindo canais de rádio, sem prejuízo da sua segurança, necessitando apenas da geração de uma chave de sessão, tarefa realizada pelo protocolo de DCQ, em taxa equivalente a taxa de transferência de dados requisitada pelo canal de comunicação.

\subsection{IDCQ para aplicações S/S}

Esta seção tem como objetivo descrever um cenário básico para a utilização do mecanismo DCQ para aplicações do tipo S/S, como por exemplo, duas sub-redes da ATN. Esse procedimento pode ser feito por meio dos passos:

Passo 1 A IDCQ distribui uma chave de sessão para dois roteadores de duas sub-redes da ATN, neste caso os pontos finais do protocolo DCQ;

Passo 2 Estas duas sub-redes da ATN utilizam a chave fornecida para proteger as mensagens que serão transitas através da Internet por meio da manutenção de uma Virtual Private Network (VPN) que utiliza a Cifra de Vernam como mecanismo 
de encriptação de dados.(ELLIOT, 2002).

A figura 27 resume o cenário apresentado.

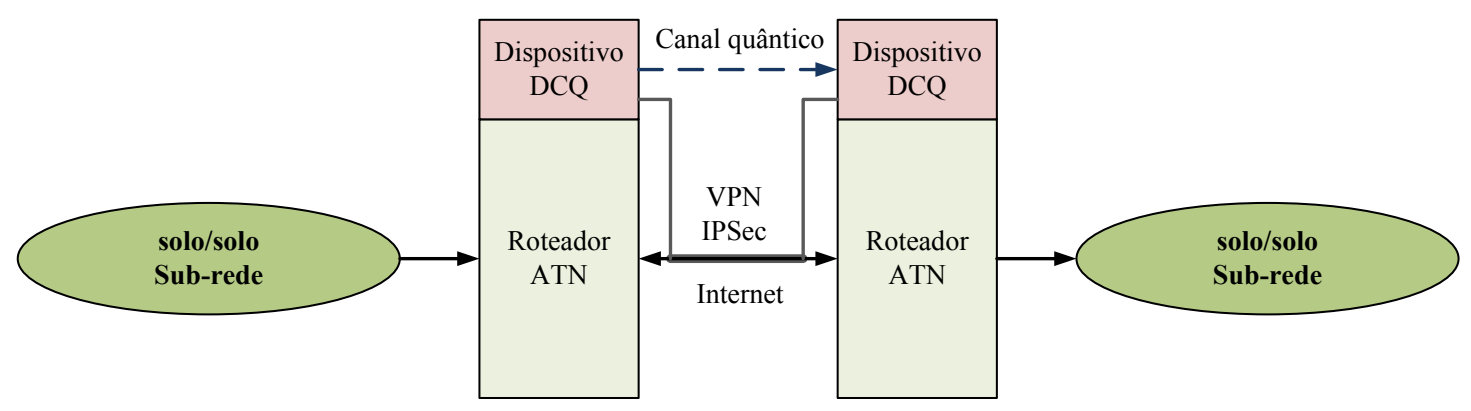

Fig. 27: IDCQ aplicação solo/solo

Em aplicações S/S a IDCQ será o mecanismo responsável por estabelecer uma chave de sessão entre duas sub-redes da ATN correspondente a Estações de Solo (GS). As vantagens para o caso de aplicações deste tipo é a utilização de soluções similares àquelas utilizadas em comunicações através da Internet via cabeamento. Contudo, neste caso, a IDCQ substituirá a ICP clássica como mecanismo de estabelecimento de chaves, utilizando um protocolo DCQ para desempenhar a função de algoritmos clássicos como o RSA ou o algoritmo Diffie-Hellman.

Tal como ocorre em aplicações $\mathrm{A} / \mathrm{S}$, nas aplicações $\mathrm{S} / \mathrm{S}$ tem-se duas possibilidades para a arquitetura da IDCQ: IDCQ baseada em solo e IDCQ baseada em satélite.

Para o caso da utilização da IDCQ baseada em solo há ainda uma escolha adicional proveniente da possibilidade de utilização de canais baseados em fibra e canais baseados em espaço aberto. Como requisito para IDCQ baseada em fibra, deve ser possível utilizar a infraestrutura de fibra já disponível e as estações de repetição clássicas e suas distâncias características.

Na figura 27 é mostrada a forma mais simples de comunicação segura baseada em uma IDCQ entre duas sub-redes da ATN. A forma mais prática de se obter o cenário proposto na figura consiste na utilização de um protocolo DCQ para estabelecimento de uma chave de sessão, trafegando dados por uma conexão TCP/IP tradicional utilizando uma VPN com segurança mantida pela Cifra de Vernam e a chave estabelecida por meio de DCQ. 
Esta é a mais frequente arquitetura encontrada em projetos de implementação de segurança em redes utilizando criptografia quântica. A primeira rede quântica, a DARPA Quantum Network, discutida anteriomente, utiliza o mecanismo de DCQ para fazer a renovação de chaves de uma VPN (Virtual Private Network) (ELLIOT, 2002). Esta arquitetura permite as aplicações operarem transparentemente uma vez que um elemento que se insere na rede tem todos os privilégios de um usuário de uma Local Area Network (LAN). Desta forma a DARPA Network é um bom exemplo de funcionamento do cenário proposto.

Os encaminhadores de dados DCQ discutidos na seção 6.1 também se mostram úteis nas aplicações S/S para contornar as limitações de distâncias máximas possíveis para realização de criptografia quântica em fibra. Os encaminhadores podem ser utilizados de duas formas, presentes em estações de solo como mostrado na figura 28 ou presentes em satélites como mostrado na figura 29.

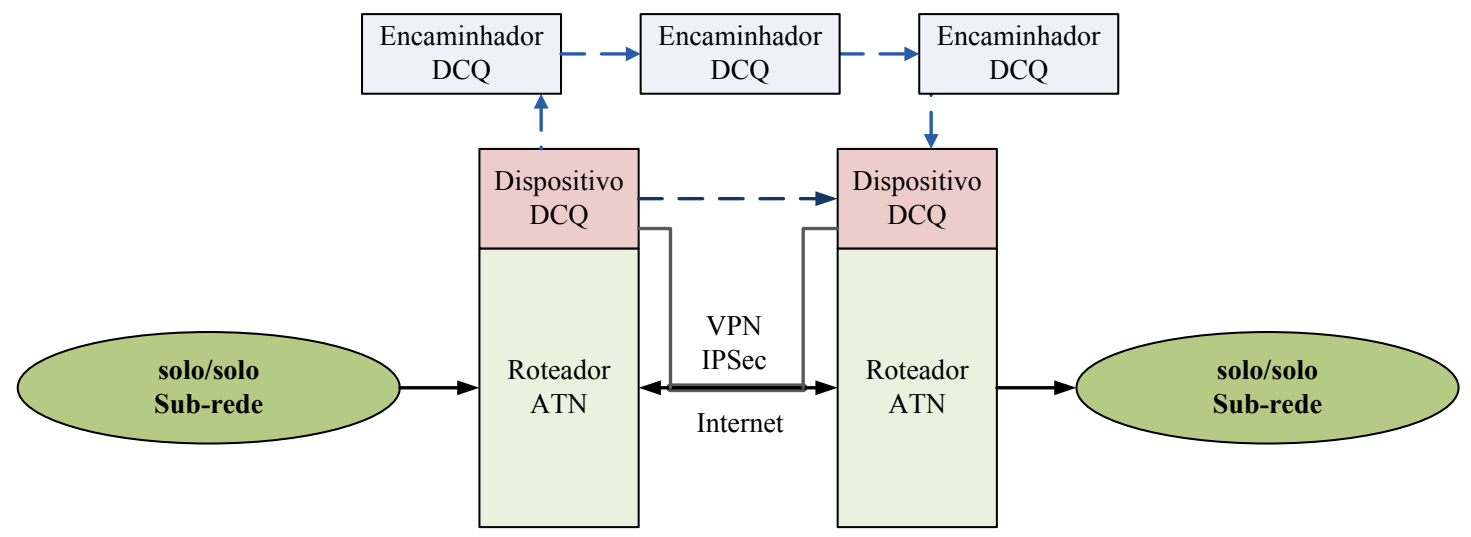

Fig. 28: IDCQ baseada em encaminhadores de dados DCQ em solo

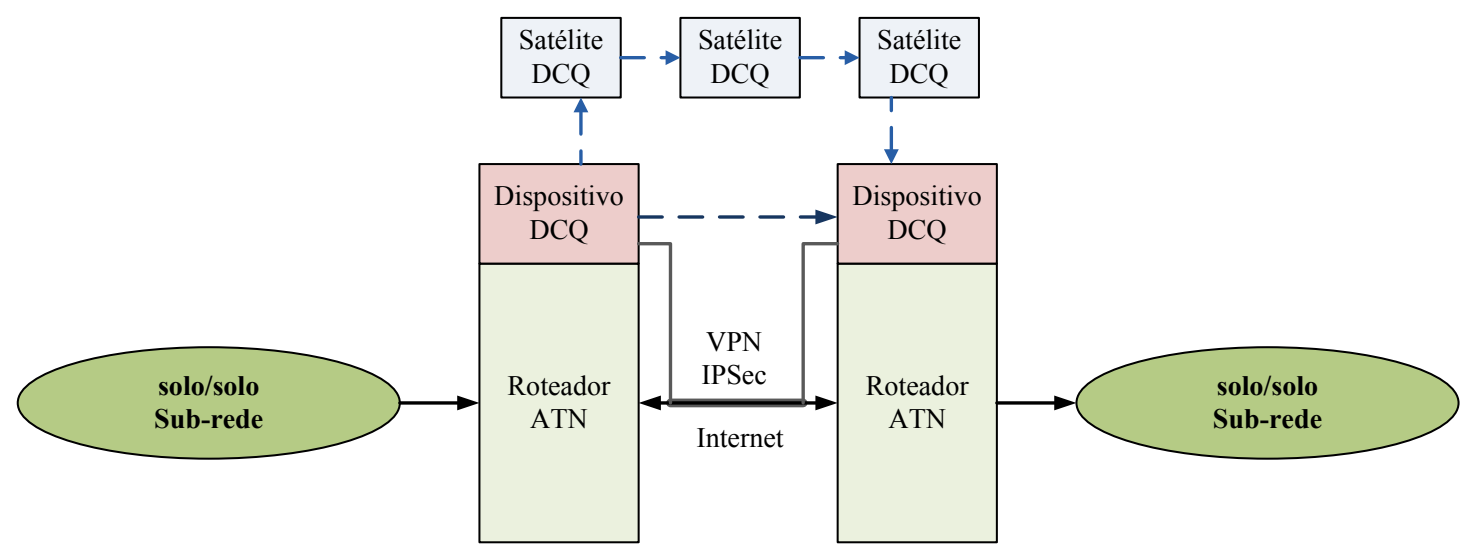

Fig. 29: IDCQ baseada em satélites agindo como encaminhadores de dados DCQ 
Apesar da grande utilidade dos encaminhadores de dados DCQ, estes introduzem uma grande limitação devido a sua necessidade de um canal ponto-a-ponto, permitindo um ataque simples de Negação de Serviço (Denial of Service) por meio da interrupção do canal onde se estabelece a DCQ. Considerando um ambiente onde a disponibilidade dos canais são requisitos fundamentais como no caso aeronáutico, essa limitação pode ser contornada por meio da disponibilização de alternativas para o estabelecimento de DCQ dentro de uma IDCQ. A figura 30 mostra como é possível operar segundo essa perspectiva, onde ocorre a interrupção de algumas rotas de comunicação e a mudança de rota dinamicamente.

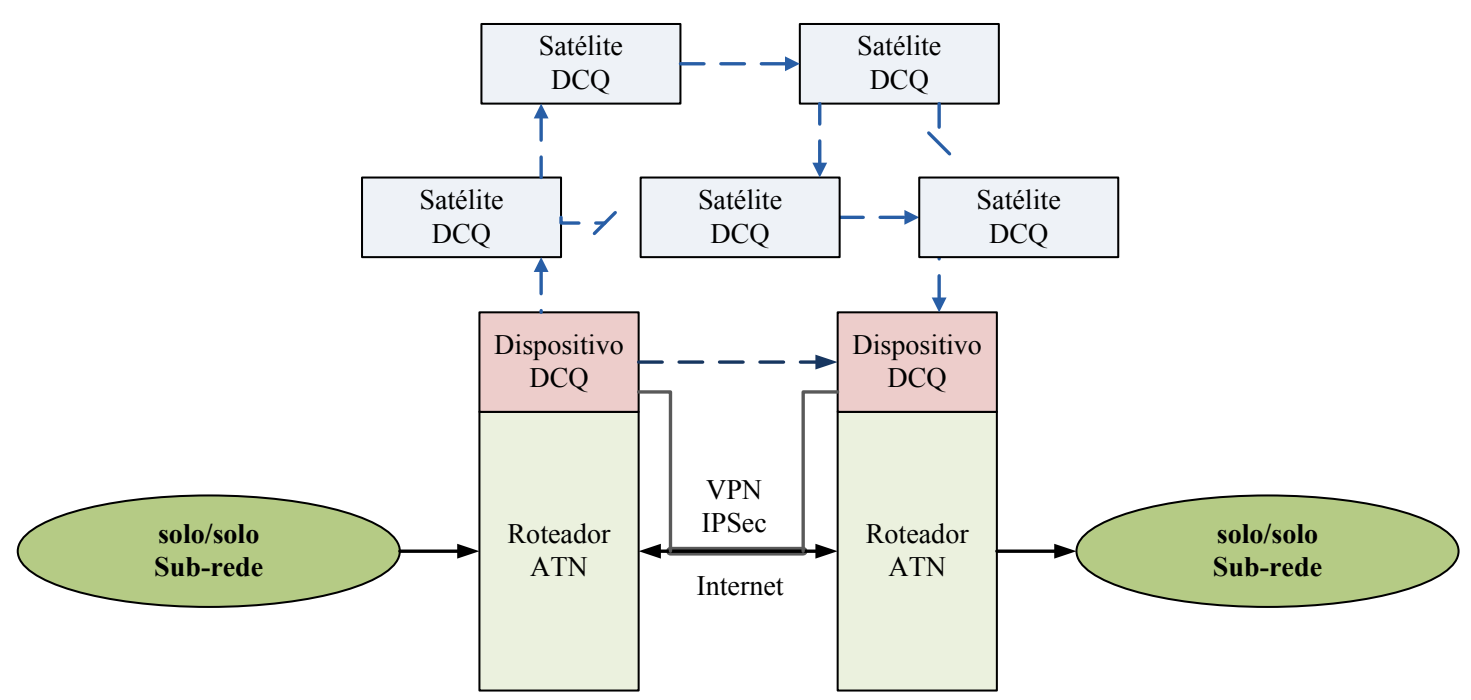

Fig. 30: IDCQ baseada em satélites agindo como encaminhadores de dados DCQ com múltiplas rotas

\subsection{Considerações finais do capítulo}

Como discutido nas seções anteriores, a utilização de satélites, apesar de representar um alto investimento, representa uma das poucas formas de se obter e manter uma solução única para o estabelecimento de mecanismos DCQ estáveis e de longo alcance. Os dados apresentados na seção 4.2.2 indicam ser possível a obtenção de uma rede global operando com protocolos DCQ, sendo estimado para isso um prazo de 5 a 10 anos pela referência (ASPELMEYER et al., 2003). Considerando que há um grande volume de investimentos provenientes de diversos setores com o intuito da obtenção de uma rede quântica global, os altos custos envolvidos no desenvolvimento da tecnologia podem 
ser compartilhados e reduzidos tal como ocorre com a infraestrutura de comunicação utilizada hoje pela Internet.

Dentre as possibilidade de obtenção de uma IDCQ que suporte as aplicações da ATN discutidas neste capítulo, a opção de uma IDCQ baseada em satélites é aquela que se mostra mais flexível e de maior viabilidade técnica. Como um fator adicional, a construção de uma malha de satélites DCQ atenderia não somente os requisitos de operação da ATN como também a diversas outras redes de telecomunicação. Dessa forma, teria-se em operação um Quantum Bone suportando diversos serviços críticos.

A utilização de uma IDCQ na ATN, como discutido anteriormente, está condicionada ao atendimento de alguns novos requisitos. Devido a utilização da Cifra de Vernam, que é um método de cifra de fluxo, a taxa de geração da chave criptográfica deve equivalente a taxa de transferência de dados na comunicação. Neste sentindo, a aplicação ao caso do ambiente aeronáutico é interessante, já que os canais de dados desse ambiente tem taxas de transferências relativamente baixas quando comparadas aquelas verificadas em outras redes de missão crítica.

O capítulo seguinte apresenta o desenvolvimento de um ambiente de simulação para a abordagem do protocolo BB84 desenvolvida no capítulo 4, obtendo a partir disso uma estimativa de desempenho esperado para uma implementação física deste, comparando o resultado obtido com o desempenho requisitado pelos principais canais de comunicação utilizados atualmente na ATN e indicando se a abordagem em questão é viável, com a tecnologia atual, como mecanismo de DCQ para a ATN. 


\section{Obtenção de um ambiente de simulação para criptografia quântica na aeronáutica}

Os benefícios decorrentes da utilização da criptografia quântica para manutenção da segurança de redes motivou diversos institutos e empresas a promoverem grandes investimentos no desenvolvimento e construção de dispositivos para implementar protocolos de distribuição de chaves quânticas, como apresentado no capítulo 4. Paralelamente a esse fato, tem sido verificado nos últimos anos o desenvolvimento de alguns trabalhos envolvendo a simulação computacional de protocolos de criptografia quântica na tentativa de prever o comportamento de futuras tecnologias de implementação. Na atual fase de desenvolvimento das técnicas de criptografia quântica surge um novo desafio, a análise da integração de técnicas de distribuição de chaves quânticas em estruturas de segurança da informação existentes. O estudo de caso proposto no capítulo anterior é uma tentativa de contribuir neste sentido.

Por outro lado, as pesquisas com foco no desenvolvimento da Rede de Telecomunicações Aeronáuticas (ATN) e técnicas de implementação que atendam a determinados requisitos de segurança (safety) são muitas e com grande diversidade de abordagens. Diversos estudos têm sido feitos com o intuito de mensurar o impacto que aspectos relacionados à disponibilidade, confiabilidade, dentre outros, têm sobre a segurança (safety) de vôo. Avaliar o atendimento de requisitos de segurança (safety) em implementações específicas da ATN não faz parte do foco deste trabalho. Contudo, a discussão realizada nos capítulos anteriores mostra que, tal como ocorre com outras redes de telecomunicações que utilizam métodos clássicos de criptografia, a ATN pode estar vulnerável do ponto de vista da segurança da informação. Este fato representa um novo requisito de segurança (safety), como discutido em capítulos anteriores. O desenvolvimento, moti- 
vado pelos resultados obtidos na implementação de protocolos de criptografia quântica, de um cenário de utilização de um protocolo de DCQ na atual especificação da ATN mostrou que esta abordagem pode ser uma alternativa viável.

O protocolo BB84 e os desenvolvimentos propostos na seção 4.1.2 podem ser avaliados como mecanismo de segurança da informação da ATN, operando de acordo com o cenário de integração proposto em 4.1.2. A viabilidade dessa abordagem está condicionada ao atendimento dos requisitos de desempenho exigidos na especificação da ATN. O desenvolvimento de um ambiente de simulação para o protocolo BB84 e os procedimentos de reconciliação de informação e amplificação de privacidade propostos no capítulo 4 em conjunto com os dados referentes às melhores implementações físicas disponíveis para protocolos de criptografia quântica permitem a obtenção de uma estimativa de desempenho para a operação do protocolo BB84 no cenário de integração com a ATN.

Este capítulo tem como objetivo apresentar o desenvolvimento de um ambiente de simulação para o protocolo de distribuição de chaves quânticas BB84 desenvolvido no capítulo 4 e sua operação no cenário de integração de um protocolo DCQ a ATN. Isso permitirá validar a proposta da utilização de um protocolo de criptografia quântica em um ambiente de telecomunicações realista, bem como avaliar o atendimento dos requisitos de desempenho exigidos pela ATN.

Para isso são apresentados na seção 7.1, a especificação e as técnicas de simulação utilizadas na implementação de um módulo de DCQ baseado no protocolo BB84 e nos desenvolvimentos apresentados no capítulo 4. Na seção 7.2 são mostrados os resultados da implementação do módulo de criptografia quântica nos cenários básicos de comunicação propostos, analisado a relação entre o desempenho do protocolo e os principais parâmetros do sistema. De acordo com as relações de desempenho obtidas e utilizando os dados de implementações realistas, é estimado o desempenho esperado para uma implementação física do módulo desenvolvido. Na seção 7.4 são contrastados os resultados de desempenho esperado na operação física do módulo de simulação para o protocolo de criptografia quântica em conjunto com a estrutura da ATN e os índices de desempenho que podem ser obtidos pela atual especificação da ATN. Em seguida é apresentada uma discussão sobre os impactos da utilização de técnicas quânticas so- 
bre este índices, limitações existentes e os desenvolvimentos ainda necessários para sua utilização prática.

\subsection{Implementação computacional de um protocolo de criptografia quântica}

A teoria apresentada no capítulo 4 refere-se à implementação de um método criptográfico baseado em um sistema físico. A implementação de um ambiente de simulação deve considerar fundamentalmente este aspecto. Assim, para a obtenção do módulo de simulação para o protocolo quântico desenvolvido anteriormente é necessário a definição da técnica de simulação para diversos parâmetros físicos.

A definição dos parâmetros utilizados na abordagem proposta são apresentados a seguir, sendo fundamentados principalmente na proposta de implementação experimental de protocolos de distribuição de chaves quânticas apresentada em (BENNETT et al., 1992).

Os parâmetros de simulação se referem a três atores principais, Alice, Bob e Eva. Como já descrito, o sistema utiliza dois canais de comunicação, o canal clássico e um canal quântico, podendo estes serem considerados como dois outros atores na implementação.

Será utilizada para a implementação dos atores participantes do protocolo a linguagem de programação $\mathrm{C}++$. As principais funções a serem implementadas são discutidas nas próximas sessões segundo esta perspectiva. A nomeação dos parâmetros utilizados na descrição a seguir será feita em acordo com aquela utilizada na codificação das funções computacionais (ver apêndice $\mathrm{C}$ para uma descrição detalha do escopo das funções computacionais implementadas e as variáveis utilizadas por estas), sendo indicado entre parênteses a notação equivalente utilizada na descrição teórica do capítulo 4. 


\subsubsection{Definição de parâmetros relevantes}

\subsubsection{Parâmetros do canal quântico}

Um canal quântico real introduz invariavelmente ruído na comunicação devido a imperfeições inevitáveis de construção. Um ambiente de simulação deve levar em consideração esse fato, sendo utilizado um fator de eficiência do canal quântico que implica em uma taxa de erro físico do canal referenciada no ambiente de simulação por physicalRate. Além disso, a intensidade de transmissão de fótons no canal quântico deve ser considerada, uma vez que a transmissão de fótons individuais ainda é uma tarefa experimental complexa, como discutido anteriormente, sendo por isso utilizado um pulso contendo alguns poucos fótons. Assim, é definido um fator de intensidade de feixe $\mu$ para o canal quântico (ver seção B.4 para maiores informações sobre feixes de luz).

\subsubsection{Parâmetros Alice}

A simulação do comportamento de Alice no protocolo exige a utilização de diversos parâmetros. O primeiro destes consiste no dimensão bruta de informação inicial, rawKey $\left(s_{A}\right)$, indicando o tamanho inicial da sequência de qubtis a serem enviadas por Alice.

Após a etapa de anúncio de bases do protoclo BB84 (seção 4.1), a chave simples, sifetdKey $\left(k_{A}\right)$ é obtida. O protocolo exige que uma porção desta, testSize $\left(s_{A} / 2\right)$, seja tomada aleatoriamente para a realização da estimativa de erro, que produz uma taxa de erro erroRate dada por:

$$
\text { erroRate }=\frac{\text { testSize }}{\text { rawKey }} \text {. }
$$

Na fase de Reconciliação é realizado um esquema interativo de correção de erro por paridade. Para a simulação, um bloco inicial de tamanho $k_{0}$ que é utilizado para o teste de paridade deve ser definido, assim como o número de iterações, antes da execução do protocolo BB84. Uma boa estimativa para o tamanho do bloco inicial pode ser obtida de forma empírica, como apresentado em (BENNETT et al., 1992), por meio da expressão: 


$$
k_{0}=\frac{1}{\text { erroRate }+\frac{1}{4 \text { erroRate }}} .
$$

O tamanho do bloco $k_{i+1}$ é definido como $k_{i+1}=2 k_{i}$. A última iteração ocorre quando o tamanho do bloco excede $1 / 4$ do tamanho total da sequência de bits. Após isso são realizados $p$ passos adicionais com um bloco de tamanho $N / 4$, sendo que esta razão nunca excede $1 / 4$ do total de bits. $O$ valor de $p$ é definido a partir da segurança que se espera para o protocolo, i.e., da definição de um valor para o parâmetro de segurança $s$ discutido na seção 4.1.2.6. O procedimento para a definição de $p$ a partir de $s$ será mostrado na seção 7.2.

\subsubsection{Parâmetros Bob}

Os parâmetros relacionados a Bob referem-se essencialmente as características relacionadas ao seu detector de fótons. Este detector, tal como o canal quântico, apresenta uma eficiência relacionada a sua capacidade de detectar fótons adequadamente, dada por $D_{e f}$. Uma outra característica importante é o que é conhecido como dark count para um detector de fótons, situação em que este apresenta uma detecção sem ter recebido nenhum fóton. A consideração da existência desse fenômeno será feita por meio da manutenção no ambiente de simulação de um nível de ruído intrínseco à execução do protocolo, sendo este referente a soma de todos os erros gerados pelas imperfeições dos equipamentos utilizados em uma implementação física do protocolo.

\subsubsection{Parâmetros Eva}

Os parâmetros de Eva estão relacionados aos tipos de ataques envolvendo a manipulação de fótons que podem ser executados por ela (seção 4.1.3). O parâmetro beamSplittRate indica quanto do total de pulsos recebidos por Eva serão divididos e o parâmetro mirrorStrenghtRate indica à proporção de números de fótons em um pulso a serem divididos. Estes dois parâmetros se relacionam aos ataques do tipo beamsplitting (divisão de feixe). Há ainda dois parâmetros que se relacionam aos ataques do tipo interceptação-reenvio. O parâmetro interferenceRate está relacionado e a proporção do feixe a ser interceptado, enquanto o parâmetro interferenceIntensity indica a intensidade de reenvio. 


\subsubsection{Definição da abordagem matemática para simulação}

\subsubsection{Estimativa da informação capturada por Eva}

Na etapa de amplificação de privacidade é escolhida publicamente uma função de Hash $h(x)$ da classe $\left\{0,1^{n}\right\} \rightarrow\left\{0,1^{n-l-s}\right\}$, com $n$ sendo o tamanho da sequência obtida no processo de reconciliação, $l$ o número máximo de bits conhecidos por Eva e $s$ um parâmetro de segurança tal que a informação máxima conhecida por Eva após a execução do processo de amplificação de privacidade é menor que $m$, tal que:

$$
m=2^{s} / \ln 2,
$$

, sendo $0<s<n-l$.

O número de bits que são divulgado e capturados por Eva pode ser estimados por meio da aproximação da informação que vaza em ambos mecanismos de ataque, beamsppliting e interceptação/reenvio. Para o caso de bits vazados por meio do ataque de interceptação/reenvio, tem-se que o número de bits capturados $\xi$ é dada por:

$$
\xi=\frac{4}{\sqrt{2}} N e+5 \sqrt{4+2 \sqrt{2} N e},
$$

com $N$ sendo o tamanho da sequência inicial após o anúncio de bases e $e$ a taxa de erros no canal quântico.

No ataque de beam-sppliting é assumido que cada pulso tem intensidade média $\mu \mathrm{e}$ que estes serão divididos com uma taxa de sucesso de $100 \%$. Isso é feito para considerar o pior caso possível, aumentando a segurança do método como um todo. Com isso, no pior caso, o número de bits $\sigma$ vazados por meio desse ataque é dado por:

$$
\sigma=N \mu+5 \sqrt{N \mu(1-\mu)}
$$

com $N$ sendo o tamanho da sequência inicial após o anúncio de bases e $\mu$ a intensidade média do feixe de fótons. O segundo termo na equação refere-se a consideração do pior caso em que Eva tem mais sorte que a média no seu ataque, permitindo-se assim um desvio-padrão igual a 5 . 
A quantidade máxima da informação obtida por Eva é limitada superiormente por:

$$
\rho=\mu+(2 \sqrt{2}) e
$$

$\operatorname{com} \mu$ sendo a intensidade média do feixe de fótons e $e$ a taxa de erros no canal quântico, i.e., $e=t / N$. Com isso, a informação $l$ obtida por Eva é definida por:

$$
l=N \rho+5 \sqrt{N(\mu(1-\mu)+(4+2 \sqrt{2}) e)}
$$

com $N$ sendo o tamanho da sequência inicial após o anúncio de bases, $\mu$ a intensidade média do feixe de fótons e $e$ a taxa de erros no canal quântico. Mais uma vez, considerando o pior caso, o segundo termo na equação refere-se a consideração de um desvio-padrão igual a 5 .

\subsubsection{Simulação do canal quântico}

As principais propriedades da criptografia quântica referem às características do canal quântico. Assim, a implementação do canal quântico foi realizada com bastante critério. Como discutido no capítulo 3, os fótons no canal quântico estão sujeitos a ação do Princípio da Incerteza de Heisenberg (ver seção B.5), o que implica que é impossível observar um estado quântico sem afetá-lo. Dessa forma é necessário o estabelecimento de uma função que execute o colapso de estados na realização de uma medida. A técnica utilizada na simulação do colapso de estados será discutida na seção sequinte, 7.1.3.

Um outro aspecto importante na construção do ambiente de simulação é a consideração da transmissão de pulsos de luz no lugar de fótons individuais, como ocorre no caso realista. Para a simulação da intensidade $\mu$ do feixe de fótons será utilizada a distribuição de Poisson (ver seção B.4.1), por meio da qual é possível definir se há ao menos um fóton ou não em um dado feixe. A implementação desse procedimento ocorre de forma simples: após Alice enviar o pulso a Bob, de forma a produzir uma média de $\mu$ fótons, este pulso alcança o detector de Eva e Bob, onde é calculado a presença de um ou mais fótons por meio da distribuição de Poisson.

A equação 7.8 é utilizada para calcular a probabilidade de $x$ ocorrências na distribuição de Poisson para uma média de intensidade de fótons $\mu$. 


$$
f(x)=\frac{\mu^{x} \cdot e^{-\mu}}{x}
$$

A equação 7.9 retorna a possibilidade de ao menos uma ocorrência para a mesma distribuição de 7.8.

$$
P(x \geq 1)=1-p(0)=\frac{\mu^{0} \cdot e^{-\mu}}{0 !}=1-e^{-\mu}
$$

\subsubsection{Obtenção de dados aleatórios}

A necessidade da obtenção de geradores de números aleatórios não-determinísticos tem ganhado importância crescente. Isso ocorre devido ao papel fundamental que sequências aleatórias desempenham nos sistemas criptográficos modernos, tanto clássicos como quânticos, em simulações numéricas envolvendo o Método Monte Carlo, pesquisa estatística, dentre outros (STIPCEVIC; ROGINA, 2006).

Historicamente se observa duas abordagens distintas na obtenção de números aleatórios: algorítmica (pseudoaleatória) e física (não-determinísitca). A primeira dessas consiste na obtenção de algoritmos geradores pseudoaleatórios que implementam uma função matemática determinística, produzindo uma sequência de números periódica determinada por um estado inicial conhecido como semente (RITTER, 1991). Esta abordagem tem sido uma alternativa de baixo custo de implementação, sendo amplamente utilizada e motivando o desenvolvimento de bons algoritmos que se mostram úteis em diversos casos. Contudo, tais geradores não são verdadeiramente aleatórios, uma vez que a entropia de uma sequência pseudoaleatória é finita e limitada superiormente pela entropia associada ao algoritmo e a semente utilizados (STIPCEVIC; ROGINA, 2007).

Uma segunda abordagem é obtenção de sequências aleatórias baseadas em sistemas físicos. Diversos métodos tem sido utilizados nesta abordagem, sendo os principais: a utilização de medidas relacionadas a efeitos físicos como: efeitos macroscópicos subjacentes de processos de mecânica estatística, ruído térmico, decaimento radioativo, ruído atmosférico ou amostragem de um processo não-linear com comportamento caótico sensível às condições iniciais. Contudo, o único método com aleatoriedade demonstrada consiste na utilização de sistemas quânticos (STIPCEVIC; ROGINA, 2007). 
A obtenção de um ambiente de simulação para protocolos de criptografia quântica é um exemplo de caso em que há a necessidade da utilização de geradores de números aleatórios, sendo a qualidade dos resultados produzidos pela simulação fortemente dependentes destes. Isso ocorre por duas razões. Primeiramente, a implementação computacional de sistemas quânticos, como a simulação do comportamento da polarização do fóton, necessita de uma gerador aleatório para implementar a natureza probabilística da mecânica quântica. Com isso, simulações quânticas realistas necessitam de uma fonte de probabilidade verdadeiramente aleatória baseada em alguma medida de sistema quântico real. Um segundo ponto envolve a simulação de protocolos quânticos. Em tais protocolos a qualidade das sequências aleatórias utilizadas nas etapas de protocolos como o BB84, são fundamentais para a segurança do sistema. Como demonstrado em (BENNETT et al., 1992), o protocolo BB84 por exemplo, se torna totalmente vulnerável se algum tipo de padrão de recorrência for observado nas sequências ou funções aleatórias.

Uma abordagem bastante eficiente na geração de números aleatórios por meio de medidas físicas em sistemas quânticos simples é apresentada em (STIPCEVIC; ROGINA, 2007). A técnica desenvolvida no Institut Ruder Boskovic, Croácia, utiliza a emissão fotonica em semicondutores na obtenção de um dispositivo de geração de números aleatórios. A qualidade da sequências geradas é demonstrada pela validação destas por meio do pacote de testes desenvolvidos pelo National Institute of Standards and Technology (NIST), Estados Unidos, para avaliar geradores de números aleatórios (STIPCEVIC; ROGINA, 2007).

O Institut Ruder Boskovic mantém por meio do Centre for Information and Computing o serviço de obtenção de números aleatórios remotamente, Quantum Random Bits Generator (QRBG) (STIPCEVIC; ROGINA, 2006). Este serviço permite a usuários cadastrados a obtenção de sequências aleatórias baseada no dispositivo apresentado em (STIPCEVIC; ROGINA, 2007) sob demanda por de comunicação remota utilizando uma classe $\mathrm{C}++$ disponibilizada no site do serviço.

Com o intuito de tornar a simulação desenvolvida neste trabalho o mais realista possível, todas as funções que necessitam de informação aleatória é baseada no serviço QRBG do Institut Ruder Boskovic. Isso é feito por meio da inclusão e adaptação da 
classe disponibilizada pelo instituto aos códigos desenvolvidos no trabalho e que são discutidos em seguida.

\subsubsection{Implementação do colapso de estados}

A natureza do canal quântico é representada pelo princípio da Incerteza de Heisenberg (ver apêndice B.4), que é essencialmente probabilístico. Dessa forma, a medição sobre um estado quântico leva a obtenção de um dos resultados possíveis, tal como discutido na seção 3.1. A natureza probabilística da mecânica quântica é implementada por meio da função aleatória disponibilizada pelo serviço QRBG. Dessa forma, todas as medidas quânticas realizadas pelo módulo de criptografia quântica tem a definição do colapso do estado quântico definido por meio de uma função probabilística baseada em um sistema quântico real.

\subsubsection{Escopo das funções de implementação}

Nesta seção são mostrados os conceitos utilizados no desenvolvimento de algumas funções computacionais, cujo objetivo consiste em obter um ambiente de simulação realista para o protocolo de criptografia quântica baseado no protocolo BB84 e nos desenvolvimentos teóricos realizados anteriormente. Esse intuito é alcançado por meio da simulação das ações de Alice, Bob e Eva e dos canais entre eles. A seguir são discutidas as definições utilizadas na implementação das funções de simulação para esses diferentes atores, utilizando a mesma notação para as variáveis de simulação verificada na codificação das funções. O código referente a definição do escopo das funções é mostrado em maiores detalhes no apêndice C.

\subsubsection{Alice}

Alice é quem inicia o protocolo, devendo se comunicar com Bob de forma a estabelecer uma chave aleatória com segurança. Isso é feito por meio do procedimento descrito na seção 4.1 .

Em um primeiro passo, Alice deve preparar a sequência inicial de qubits aleatórios. Tal ação pode ser implementada por meio da função randomsequence, que gera duas 
sequências de bits aleatórias de tamanho rawSize referentes a chave a ser estabelecida e a sequência de bases escolhidas na codificação dos qubits. Alice deve então implementar essa codificação por meio da produção do feixe a ser enviado a Bob, etapa na qual deve ser considerado a limitação das técnicas de implementação atuais, que ocasionalmente produzem mais de um fóton por pulso. A função photonSource, utilizando a distribuição de Poisson, indica para uma dada intensidade de feixe escolhida em quais pulsos produzidos por Alice há mais de um fóton.

Após o término do recebimento da sequência de qubits por Bob, Alice deve então anunciar publicamente a sequência de bases utilizadas no processo de codificação para que, juntamente com Bob, elimine os bits em que Bob utilizou uma base diferente para realizar a medição. Essa ação consiste no segundo passo e é realizada por meio da função basesVerification, que executa a comparação entra as bases anunciadas por Alice e Bob e retorna as posições em que a medida de Bob foi realizada na base correta, gerando a sequência intermediaria siftedKey.

O terceiro passo realizado por Alice consiste na verificação de erros. Para isso é utilizada a função bitsDivulged que seleciona aleatoriamente um subconjunto de tamanho testSize da sequência siftedKey e compara publicamente os valores correspondentes entre os subconjuntos de Alice e Bob. Com isso é calculado e erro observado no subconjunto por meio de equação (7.1). Os bits anuciados no processo são descartados após esta etapa.

No quarto passo Alice e Bob, possuem privadamente suas sequências siftedKey, que devido ao ruído introduzido na comunicação é parcialmente correlacionada. Para a correção de erros é realizado o processo de reconciliação por meio da variação do algoritmo Cascade proposto em 4.1.2.5. O processo é realizado por meio de três funções. A primeira função, parityCheck testa a paridade entre dois blocos de tamanho vectorPosition de sequências distintas, anunciando publicamente o bit de paridade para cada bloco. Uma segunda função, binaryAction, utilizando a função parityCheck, executa a primitiva iterativa BINARY proposta em 4.1.2.5, que localiza e encontra possíveis erros no bloco iniciado na posição vectorPosition e de tamanho blockSize. Por fim, a função biconfirmAction executa a primitiva iterativa BICONFIRM $^{s}$ proposta em 4.1.2.5, tomando um subconjunto aleatório de duas sequências 
distintas e executando a primitiva CONFIRM sobre este.

A etapa seguinte consiste na execução do procedimento de amplificação de privacidade, tal como proposto em 4.1.2.6. Para isso é utilizada a função universalHash que toma aleatoriamente um bloco de bits de tamanho randomBlock da chave estabelecida após o procedimento de reconciliação de informação, calcula sua paridade e registra o valor, executando este processo um número de vezes igual a hashSize. A chave final obtida tem tamanho igual a hashSize, sendo a relação entre estes valores mostrada na seção 7.2

\subsubsection{Bob}

Sendo Bob a contraparte de Alice no protocolo, algumas funções utilizadas para descrever sua ação são semelhantes aquelas utilizadas para Alice.

No primeiro passo, Bob deve obter uma sequência aleatória para as bases que utilizará na medição dos qubits enviados por Alice, fazendo isso por meio da função randomSequence. Em um segundo passo, Bob realiza a medição dos estados enviados por Alice de acordo com as bases obtidas anteriormente, fazendo isso por meio da função bitColapse que implementa o colapso de estados probabilisticamente. Como resultado da operação Bob obtém a sequência siftedKey.

Os passos seguintes executados por Bob são equivalentes aqueles executado por Alice, utilizando por isso as mesmas funções descritas anteriormente para Alice, considerando como entrada as correlacionadas mais ainda distintas sequências siftedKey que Alice e Bob pretendem compartilhar.

\subsubsection{Eva}

Eva age entre Alice e Bob tentando adquirir o máximo de informação possível da chave compartilhada entre eles. Dessa forma, as ações relacionadas a Eva estão associadas aos possíveis ataques que podem ser efetuados contra o protocolo quântico. A primeira abordagem consiste na interferência ativa na sequência de qubits enviada por Alice, representada por uma medição dos qubits capturados e posterior reenvio a Bob. Essa ação é realizada por meio da função randomSequence, que neste caso é uti- 
lizada para executar uma seleção aleatória dos qubits enviados por Alice, e a função bitColapse, utilizada para definir o colapso de estados. Com o intuito de analisar o pior caso, é considerado que Eva possui um detector ideal, não inserindo erro em sua medida.

Uma segunda abordagem é representada pela execução de um ataque do tipo beamsplitting. Como visto anteriormente, este ataque está relacionado a imperfeição intrínseca das fontes de fótons desenvolvidas até o momento. Estas fontes tem uma distruição de probablidade de se encontrar mais de um fóton por pulso definida pela distrubuição de Poisson. A definição do procedimento de amplificação de privacidade, realizada na seção 4.1.2.6, trata adequadamente a existência da informação extra que é divulgada na produção de fótons, tornando esta inútil. Com isso, no ambiente de simulação é considerada uma fonte de fótons com probabilidade dada pela distrubuição de Poisson. Isso é feito por meio da função photonSource, que aplica essa distribuição à sequência de qubits produzidos por Alice.

\subsubsection{Canal}

A implementação do comportamento do canal está relacionada a eficiência com que o meio físico escolhido executa o transporte dos fótons preservando seus estados iniciais. As técnicas atuais invariavelmente inserem ruído na transmissão dos qubtis. Sendo assim, é utilizada a função randomErro que insere aleatoriamente uma taxa de erros physicalRate na sequência de fótons codificadas por Alice.

\subsubsection{Encaminhador quântico}

O elemento adicional representado pelo Encaminhador de Distribuição de Chaves Quânticas, apresentado na seção 6.1, é necessário na simulação de um cenário realista de aplicação de criptografia quântica. Como discutido anteriormente, o comportamento desse elemento é essencialmente o mesmo executado por Bob, sendo por isso utilizadas as mesmas funções daquele na obtenção do seu comportamento. 


\subsection{Simulação do protocolo BB84}

\subsubsection{Relação entre os parâmetros de simulação e desempenho}

O passo seguinte na obtenção de um ambiente de simulação realista para um protocolo de criptografia quântica consiste na análise da relação entre os parâmetros de simulação definidos anteriormente e o desempenho do protocolo. Os índices de desempenho relevantes para um protocolo quântico em uma operação realista são principalmente: taxa de codificação de qubits (Quatum Bit Rate, $Q B R$ ), taxa de erros nos qubits (Quantum Bit Error Rate, $Q B E R$ ) - sendo este parâmetro a soma de todas as fontes de erro - e taxa de geração de chave (Key Generation Rate, $K G R$ ). No caso de uma implementação física, três parâmetros principais influenciam os índices de desempenho: distância máxima de transmissão $\left(D_{\max }\right)$, intensidade de transmissão do feixe $(I)$ e a média de fótons presentes em cada pulso $(\mu)$, sendo seu impacto sobre o protocolo quântico proposto discutido ao longo da seção.

Por meio do ambiente de simulação desenvolvido é possível analisar como o índice $K G R$, que em última instância é o limitante no desempenho da comunicação, depende dos índices $Q B R$ e $Q B E R$ utilizando a proposta desenvolvida anteriormente em um caso prático. O conhecimento desta relação juntamente com a utilização dos dados relacionados a estes parâmetros obtidos pelas melhores técnicas de implementação disponíveis atualmente permitirá obter os níveis de desempenho esperados para uma implementação física das técnicas propostas neste trabalho. Adicionalmente, a análise das relações de desempenho permite verificar o comportamento do ambiente desenvolvido e aquele esperado teoricamente.

O estudo do desempenho do ambiente de simulação desenvolvido parte do cenário fundamental representado pela comunicação ponto-a-ponto no caso ideal, ou seja, a ausência de ruído e qualquer tentativa de corrompimento. Neste caso não são realizadas as etapas de reconciliação de informação e amplificação de privacidade. Este cenário permite analisar a estatística básica do protocolo e posteriormente permite uma comparação com aquela verificada em casos mais complexos.

Considerando uma sequência inicial rawKey de $\mathbf{1 0 2 4}$ bits, o resultado da execução do protocolo sucessivamente para 15 casos é apresentada na tabela 4 . 
Tab. 4: Realizações do protocolo BB84 em um cenário ideal

\begin{tabular}{ccc}
\hline Bases corretas & Bits divulgados & Erro \\
\hline \hline 519 & 259 & 0 \\
513 & 256 & 0 \\
503 & 251 & 0 \\
515 & 257 & 0 \\
502 & 251 & 0 \\
506 & 253 & 0 \\
518 & 259 & 0 \\
477 & 238 & 0 \\
492 & 246 & 0 \\
503 & 251 & 0 \\
526 & 263 & 0 \\
540 & 270 & 0 \\
511 & 255 & 0 \\
514 & 257 & 0 \\
498 & 249 & 0 \\
\hline
\end{tabular}

A figura 31 mostra o valor médio do número de bases em concordância (BC) e o tamanho da chave final obtida (SK), indicando que, como esperado pelas probabilidades envolvidas no protocolo no cenário idealizado em questão, a chave final obtida tem tamanho médio igual a 1/4 do tamanho da sequência inicial de qubits codificadas. Considerando uma taxa de codificação de bits $Q B R$, a relação de desempenho para os casos em que não são realizadas os processos de reconciliação de informação e amplificação de privacidade pode ser expressa por:

$$
\overline{K G R} \simeq \frac{1}{4} \overline{Q B R}
$$

Considerando ainda o caso idealizado de ausência de ruído, é possível verificar o impacto na estatística do protocolo que a interferência relacionada a uma tentativa de corrompimento gera. A tabela 5 mostra a estatística do protocolo para uma sequência inicial rawKey de $\mathbf{1 0 2 4}$ bits e a tentativa padrão de espionagem representada pela interferência em todos os qubits transmitidos no canal quântico para 15 realizações do protocolo.

A figura 32 mostra a distribuição dos erros encontrados na realização do protocolo por 100 vezes no caso de interferência, evidenciando a média do erro no caso de interferência completa. Esta média pode ser definida por: 


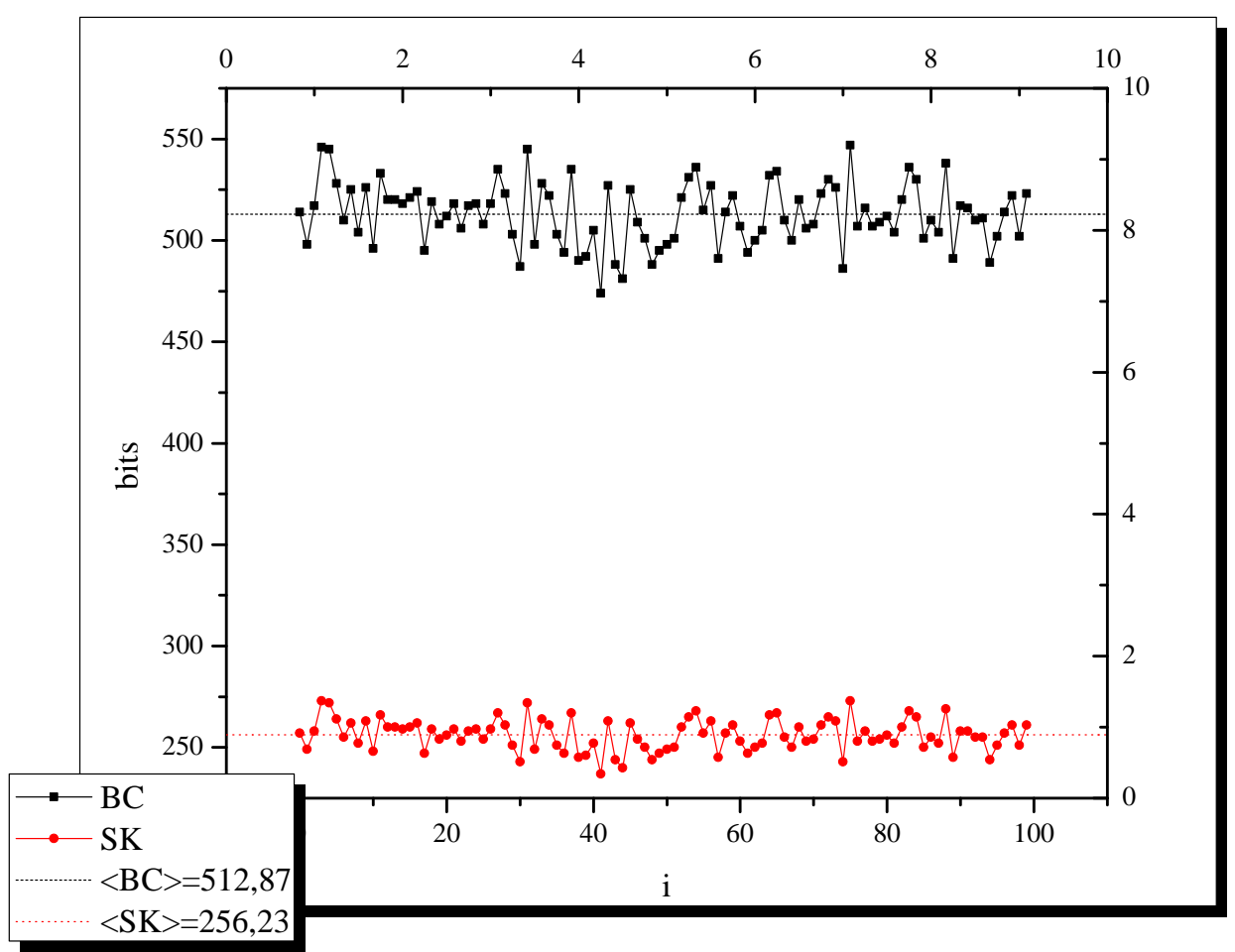

Fig. 31: Tamanho da sequência de bases corretas BC e chave final SK obtida em 100 realizações do protocolo $\mathrm{BB} 84$ com valores médios $\langle B C>$ e $\langle S K\rangle$

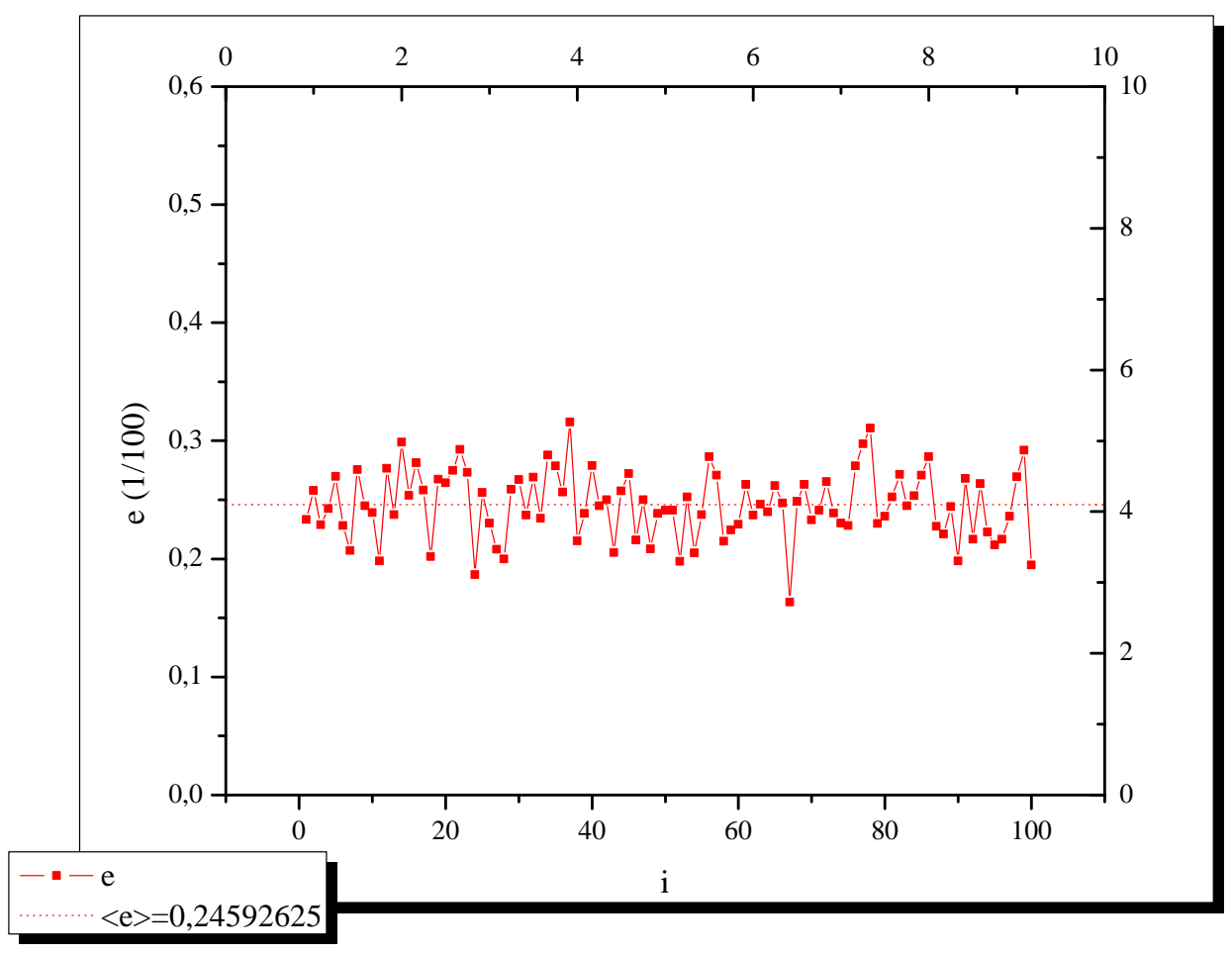

Fig. 32: Erro $e$ e erro médio $<e>$ observado em 100 realizações do protocolo BB84 na presença de interferência completa 
Tab. 5: Realizações do protocolo BB84 na presença de interferência

\begin{tabular}{ccc}
\hline Bases corretas & Bits divulgados & Erro \\
\hline \hline 519 & 259 & 0,200772 \\
513 & 256 & 0,273438 \\
503 & 251 & 0,286853 \\
515 & 257 & 0,295720 \\
502 & 251 & 0,294821 \\
506 & 253 & 0,213439 \\
518 & 259 & 0,250965 \\
477 & 238 & 0,264706 \\
492 & 246 & 0,227642 \\
503 & 251 & 0,270916 \\
526 & 263 & 0,209125 \\
540 & 270 & 0,255556 \\
511 & 255 & 0,247059 \\
514 & 201 & 0,263682 \\
521 & 202 & 0,222772 \\
\hline
\end{tabular}

$$
\overline{Q B E R} \simeq \overline{E_{m}},
$$

onde $\overline{E_{m}}$ representa o erro médio relacionado a medida de acordo com as probabilidades discutidas na seção 4.1.2. O valor médio obtido é então o valor médio esperado teoricamente para este caso: $(\approx 25 \%)$. Este é o valor utilizado como limite máximo de referência para o abandono de comunicação e reinicio do protocolo.

Os casos analisados anteriormente tem apenas caráter ilustrativo da estatística presente no protocolo, uma vez que não têm representação real devido a impossibilidade de inexistência de ruído na comunicação. É fundamental por isso, verificar o impacto que a geração de ruído na comunicação tem na estatística do protocolo. Considerando os limites máximos de ruído, discutidos na seção 4.1.2, pode-se definir uma taxa média constante para a geração de ruído aleatório e verificar o impacto deste na estatística do protocolo na ausência de interferência. A tabela 6 mostra a estatística do protocolo para 15 execuções utilizando uma sequência inicial rawKey de $\mathbf{1 0 2 4}$ bits e uma taxa de ruído gerado aleatoriamente de $\approx 5 \%$.

A figura 33 mostra a distribuição dos erros encontrados na realização do protocolo por 100 vezes no caso da geração aleatória de ruído e ausência de interferência. A partir da tabela 6 e do gráfico da figura 33 é possível verificar que a escolha aleatoriamente de 
Tab. 6: Realizações do protocolo BB84 na presença de ruído

\begin{tabular}{cccc}
\hline Erro meio físico & Bases corretas & Bits divulgados & Erro verificado \\
\hline \hline 0,0478516 & 495 & 247 & 0,0445344 \\
0,0498047 & 534 & 267 & 0,0486891 \\
0,0478516 & 528 & 264 & 0,0416667 \\
0,0517578 & 513 & 256 & 0,0625000 \\
0,0498047 & 520 & 260 & 0,0500000 \\
0,0498047 & 513 & 256 & 0,0507812 \\
0,0458984 & 515 & 257 & 0,0428016 \\
0,0498047 & 523 & 261 & 0,0421456 \\
0,0498047 & 519 & 259 & 0,0308880 \\
0,0478516 & 532 & 266 & 0,0338346 \\
0,0517578 & 516 & 258 & 0,0852713 \\
0,0498047 & 542 & 271 & 0,0405904 \\
0,0478516 & 499 & 249 & 0,0441767 \\
0,0478516 & 495 & 247 & 0,0526316 \\
0,0498047 & 510 & 255 & 0,0627451 \\
\hline
\end{tabular}

uma fração de bits como referência para verificação de erros permite a obtenção apenas de uma aproximação do erro real. Contudo, verificando os valores médios do erro físico gerado $<e_{f}>$ e do erro medido $<e_{m}>$ para 100 realizações do protocolo, percebe-se que, na média, este mecanismo de verificação de erros se aproxima bastante do erro real.

Adicionalmente é possível verificar a estatística do protocolo na presença de ruído aleatório e interferência completa. A tabela 7 mostra a estatística do protocolo para 15 execuções utilizando uma sequência inicial rawkey de 1024 bits, uma taxa e ruído gerado aleatoriamente de $\approx 5 \%$ na presença de interferência.

A figura 34 mostra a distribuição dos erros encontrados na realização do protocolo por 100 vezes no caso da geração aleatória de ruído e existência de interferência total, permitindo verificar que a inserção de ruído aumenta, proporcionalmente a sua taxa, o erro médio observado. A relação neste caso é definida por:

$$
\overline{Q B E R}=\overline{E_{m}}+\frac{1}{2} \overline{E_{n}},
$$

onde $\overline{E_{m}}$ representa o erro médio relacionado a medida $(\approx 25 \%)$ realizada na tentativa de corrompimento e $\overline{E_{n}}$ o erro médio relacionado ao ruído físico do canal. O termo $\overline{E_{n}}$ é divido por dois devido ao fato que para $50 \%$ dos casos, a escolha de uma base errada 


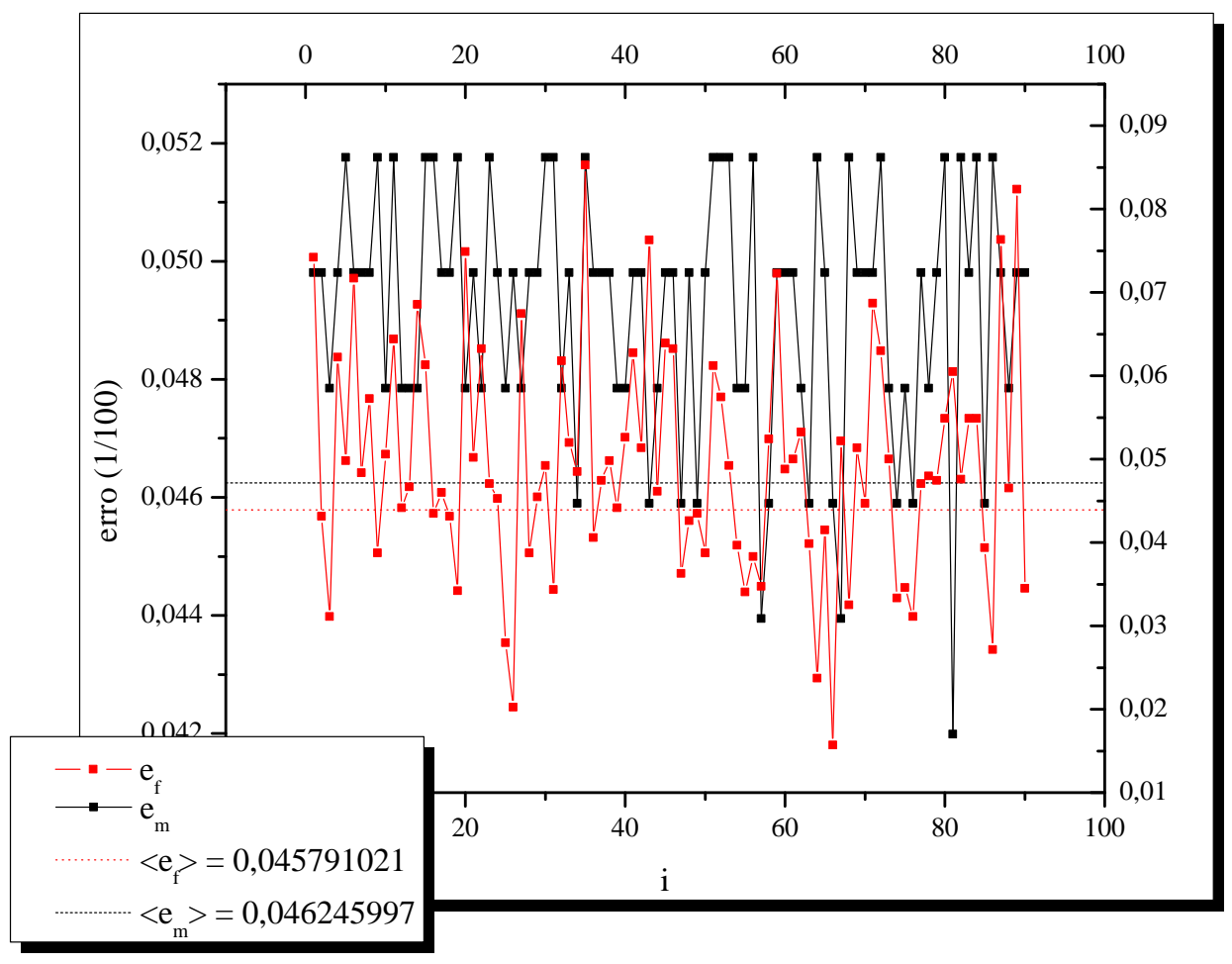

Fig. 33: Erro aleatório físico gerado $e_{f}$ e erro medido $e_{m}$ em 100 realizações do protocolo BB84 na ausência de interferência com valores médios $\left\langle e_{f}>\mathrm{e}\left\langle e_{m}\right\rangle\right.$

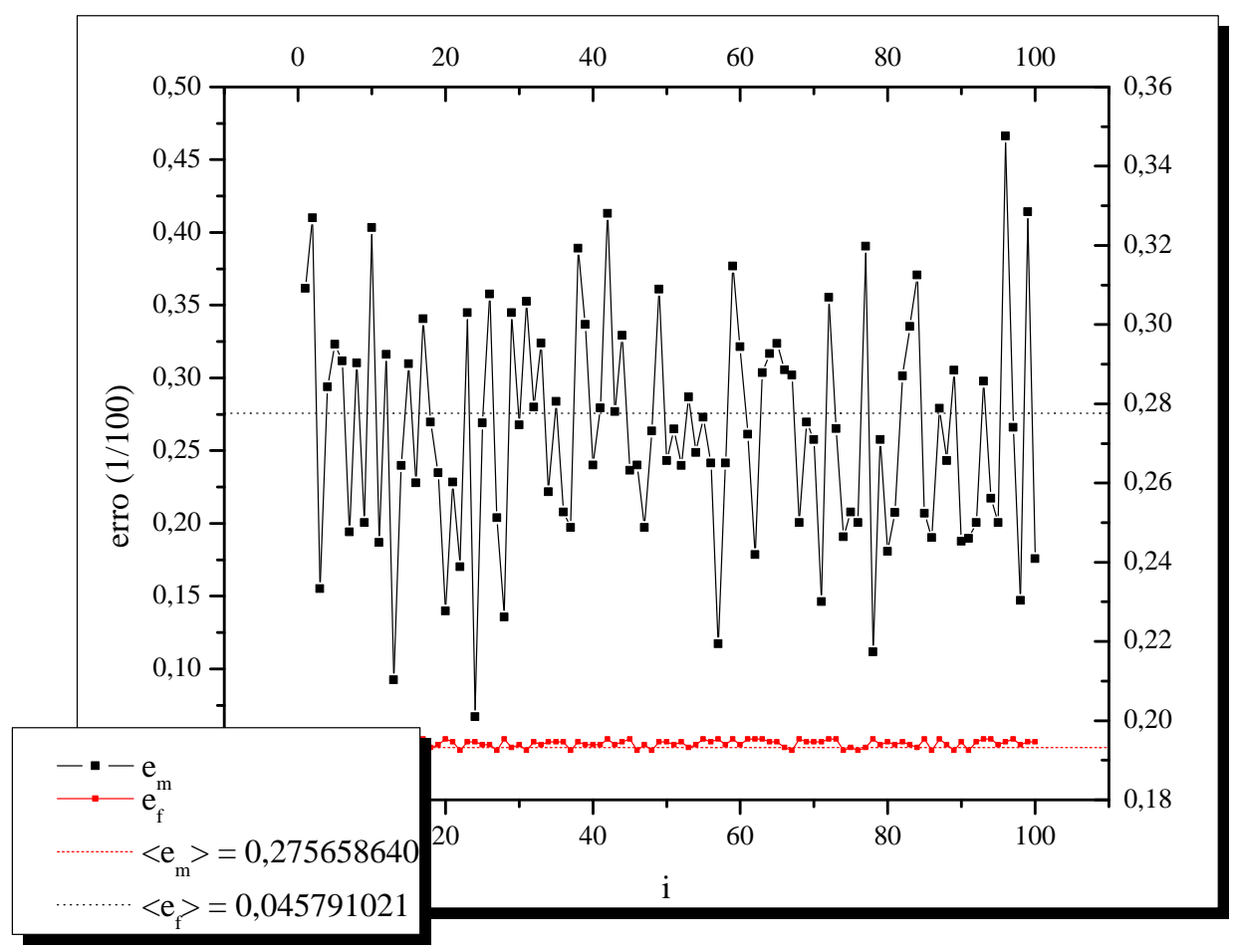

Fig. 34: Erro aleatório físico gerado $e_{f}$ e erro medido $e_{m}$ em 100 realizações do protocolo BB84 na presença de interferência total com valores médios $<e_{f}>\mathrm{e}<e_{m}>$ 
Tab. 7: Realizações do protocolo BB84 na presença de ruído e interferência

\begin{tabular}{cccc}
\hline Erro meio físico & Bases corretas & Bits divulgados & Erro \\
\hline \hline 0,0517578 & 547 & 273 & 0,271062 \\
0,0458984 & 486 & 243 & 0,283951 \\
0,0517578 & 519 & 259 & 0,223938 \\
0,0498047 & 521 & 260 & 0,288462 \\
0,0478516 & 509 & 254 & 0,267717 \\
0,0498047 & 511 & 255 & 0,333333 \\
0,0517578 & 521 & 260 & 0,276923 \\
0,0517578 & 520 & 260 & 0,311538 \\
0,0478516 & 526 & 263 & 0,258555 \\
0,0478516 & 493 & 246 & 0,292683 \\
0,0439453 & 501 & 189 & 0,264550 \\
0,0478516 & 526 & 209 & 0,248804 \\
0,0439453 & 507 & 205 & 0,273171 \\
0,0498047 & 501 & 191 & 0,308901 \\
0,0498047 & 540 & 207 & 0,265700 \\
\hline
\end{tabular}

por Eva corrige um qubit corrompido pelo ruído físico do canal. Os dados anteriores se referem ao caso de interferência completa. Contudo, a equação (7.12) também se aplica ao caso em que é adotada a estratégia de interferência seletiva, discutida na seção 4.1.2, na tentativa de se obter alguma informação parcial acerca da chave trocada. Assim, mesmo uma interferência seletiva altera a estatística esperada para o protocolo no caso da existência de apenas uma taxa de ruído esperada, tal como o caso mostrado na tabela 6. Isso permite que esse tipo de estratégia de interferência seja detectada por meio da definição de valores máximos de ruído esperados.

Os cenários tratados anteriormente evidenciam que a simulação de execução do protocolo de criptografia quântica apresenta um comportamento tal como o esperado teoricamente para os casos fundamentais. Isto é, a interferência completa causada por uma tentativa de espionagem altera a estatística do protocolo, podendo ser detectada. Mesmos nos cenários em que há a presença de ruído, quando este não ultrapassa o limite máximo de (25\%), a interferência causada por uma tentativa de espionagem pode ser percebida e tratada adequadamente. Em todos os casos, o desempenho do protocolo, representado pela taxa de geração de chave $(K G R)$, está relacionado a taxa de geração de qubits $(Q B R)$ por meio da equação (7.10).

Contudo, como discutido na seção 4.1.2, há questões não tratadas na implementação dos casos anteriores. Primeiramente, é preciso tratar os erros inerentes ao processo de 
comunicação, aspecto necessário para a geração de uma chave idêntica entre ambas as partes do protocolo. Em segundo lugar, é preciso verificar o impacto sobre o protocolo de ataques mais complexos que podem ser executados.

O tratamento de erros é realizado por meio da etapa de reconciliação de informação utilizando o algoritmo Cascade modificado, apresentado na seção 4.1.2.5 e implementado pelas funções discutidas na seção anterior. A definição dos parâmetros para o protocolo Cascade modificado é fundamentada nas relações apresentadas na seção 4.1.2.5.

Utilizando dados dos casos anteriores é possível verificar o comportamento das funções de reconciliação de erros pelo algoritmo Cascade modificado. Para o ajuste experimental dos parâmetros é realizado o algoritmo Cascade modificado para 100 execuções do protocolo. Com o intuito de verificar o erro em cada iteração do algoritmo, utiliza-se a medida de erro absoluta, comparando-se ambas as sequências de bits em sua totalidade. Este não é o processo que será utilizado na simulação de um caso realista. A tabela 8 mostra o erro verificado em cada iteração do algoritmo Cascade modificado para um caso típico, utilizando como bloco inicial $k_{0}=20$ de acordo com a equação 7.2. A figura 35 mostra a taxa de erro verificada em cada iteração do algoritmo Cascade modificado em 10 diferentes execuções do protocolo, com bloco inicial de mesmo tamanho, onde cada curva de cor diferente representa uma execução distinta.

A figura 35 indica que a implementação do algoritmo Cascade modificado permite a completa correção dos erros entre as sequências de Alice e Bob. Contudo, a verificação de erros utilizada no exemplo anterior é inviável no caso prático, já que representaria a revelação pública da chave no processo de verificação do erro. Por isso, tal como proposto na seção 4.1.2.5, Alice e Bob devem concordar previamente em uma quantidade de iterações a serem realizadas após o bloco de teste do algoritmo exceder $1 / 4$ do tamanho da sequência inicial. O exemplo anterior indica que a quantidade necessária de iterações para corrigir todos os erros não excede, no pior caso, 53 iterações para uma taxa de erro médio de $\approx 5 \%$. Encontrado os parâmetros experimentalmente mais eficientes para o algoritmo Cascade modificado, este pode ser aplicado ao caso prático do estabelecimento de uma chave na ausência de interferência e presença de ruído.

A realização da etapa de reconciliação de informação permite a correção dos erros 
Tab. 8: Execução do algoritmo Cascade

\begin{tabular}{cccc}
\hline Iteração & Tamanho do bloco $k_{i}$ & Característica do Bloco & Erro \\
\hline \hline 1 & & & 0,0359477 \\
2 & 20 & sequencial & 0,0163399 \\
3 & 40 & sequencial & 0,0163399 \\
4 & 80 & sequencial & 0,0163399 \\
5 & 76 & aleatório & 0,0163399 \\
5 & 76 & aleatório & 0,0163399 \\
6 & 76 & aleatório & 0,0130719 \\
7 & 76 & aleatório & 0,00980392 \\
8 & 76 & aleatório & 0,00980392 \\
9 & 76 & aleatório & 0,00980392 \\
10 & 76 & aleatório & 0,00653595 \\
11 & 76 & aleatório & 0,00326797 \\
12 & 76 & aleatório & 0,00326797 \\
13 & 76 & aleatório & 0,00326797 \\
14 & 76 & aleatório & 0,00326797 \\
15 & 76 & aleatório & 0,00326797 \\
16 & 76 & aleatório & 0,00326797 \\
17 & 76 & aleatório & 0,00326797 \\
18 & 76 & aleatório & 0,00326797 \\
19 & 76 & aleatório & 0 \\
\hline
\end{tabular}

entre as sequências referentes as chaves de Alice e Bob. Contudo, nesta etapa é feito o anúncio público de alguns dos bits de paridade referente a cada bloco a ser testado. Esse fato permite a Eva adquirir alguma informação sobre a chave estabelecida pelo protocolo. Dois tratamentos são necessários para fazer com que a informação capturada por Eva seja inutilizada. O primeiro destes consiste no descarte do último bit dos blocos que tiveram sua paridade anunciada, de acordo com a teoria discutida na seção 4.1.2.5. Esse tratamento reduz o tamanho da chave final obtida e altera por isso a relação de desempenho do protocolo. Nesse sentindo o protocolo Cascade modificado implementado na simulação é bastante eficiente quando comparado ao algoritmo Cascade original apresentado em (BENNETT et al., 1992), necessitando revelar apenas uma pequena fração da chave estabelecida. A fração a ser revelada depende da taxa de erro verificada antes do processo. A tabela 9 mostra a quantidade de bits revelados no processo de reconciliação de informação para 10 execuções do protocolo BB84 e a média referente a 100 execuções do protocolo.

Considerando a sequência inicial de 1024 qubits do protocolo e os valores médios mostrados na tabela 9, a relação de desempenho do protocolo utilizando a etapa de 


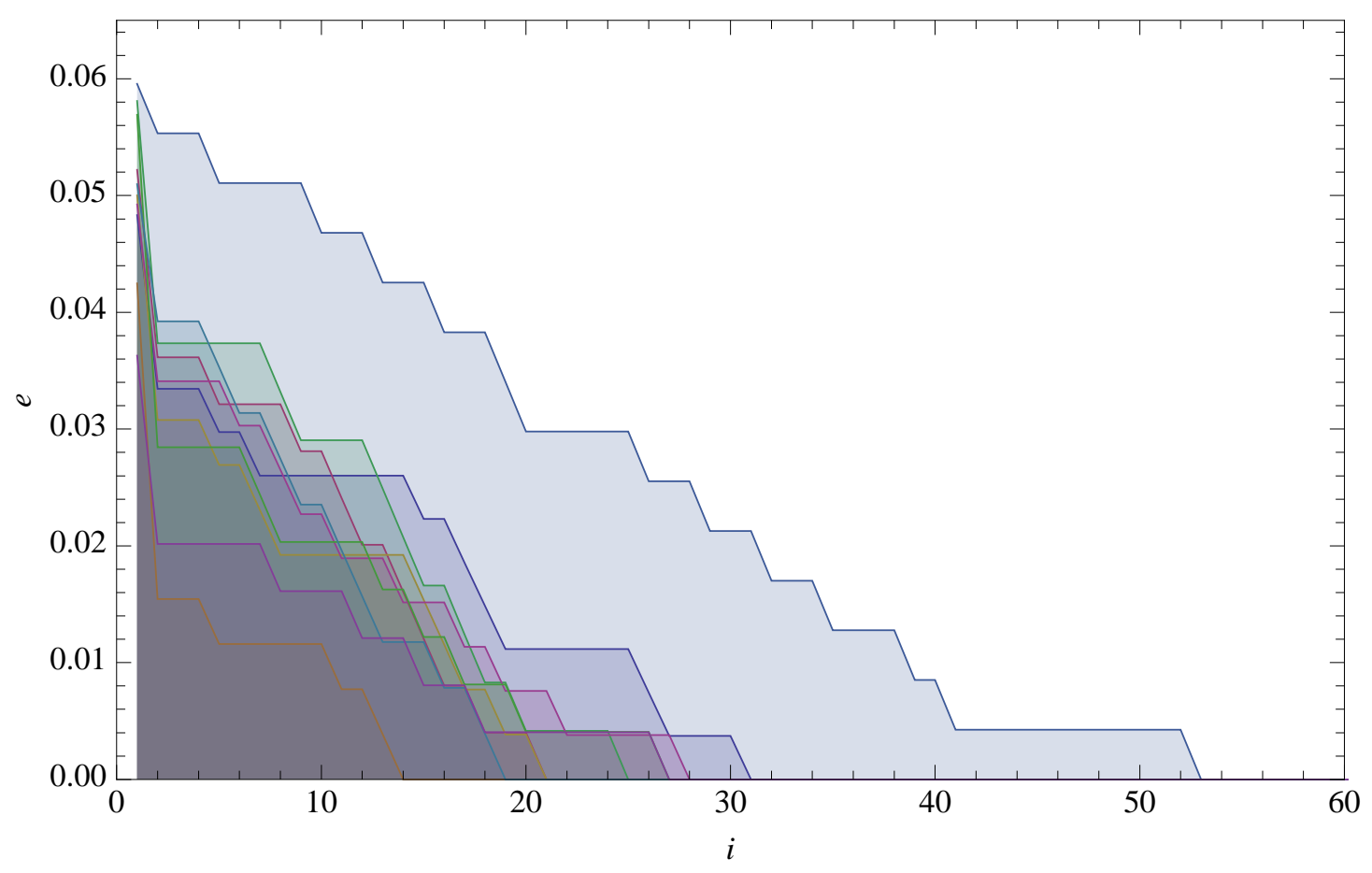

Fig. 35: Execução do algoritmo Cascade para 10 casos distintos

reconciliação de informação, considerando uma taxa média de erro de $\approx 5 \%$, é obtida da forma:

$$
\overline{K G R} \simeq \frac{1}{5} \overline{Q B R}
$$

O segundo tratamento necessário na obtenção da chave final se refere ao fato de que, mesmo eliminando os bits de paridade dos blocos testados, a divulgação desta informação faz com que Eva adquira certo conhecimento sobre a chave pelos motivos apresentados em 4.1.2.5. Essa informação pode ser eliminada no processo de amplificação de privacidade. Esse processo permite que Alice e Bob diminuam a relevância da informação capturada por Eva, com o custo de diminuir novamente o tamanho da sequência trocada e com novo impacto sobre o desempenho do protocolo.

Tal como discutido na seção 4.1.2.5, o tamanho da chave final obtida após o processo de amplificação de privacidade depende do cálculo de uma estimativa da informação capturada por Eva. Essa estimativa consiste na soma de todas as fontes de vazamento de informação para Eva, incluindo os ataques conhecidos.

O cálculo da informação que vaza por meio do ataque de beam-splitting tem trata- 
Tab. 9: Realizações do protocolo BB84 na presença de ruído

\begin{tabular}{|c|c|c|c|c|}
\hline & Erro inicial & Tamanho inicial & Bits descartados & Tamanho final \\
\hline & 0,0483271 & 269 & 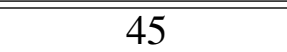 & 224 \\
\hline & 0,0522088 & 249 & 44 & 205 \\
\hline & 0,0500000 & 260 & 49 & 211 \\
\hline & 0,0580913 & 241 & 41 & 200 \\
\hline & 0,0595745 & 235 & 42 & 193 \\
\hline & 0,0492424 & 264 & 38 & 226 \\
\hline & 0,0424710 & 259 & 41 & 218 \\
\hline & 0,0569106 & 246 & 47 & 199 \\
\hline & 0,0509804 & 255 & 44 & 211 \\
\hline & 0,0362903 & 248 & 32 & 216 \\
\hline média & 0,05040964 & 252,6 & 42,3 & 210,3 \\
\hline
\end{tabular}

mento especial, uma vez que a captura desta informação não interfere no protocolo e por isso não pode ser detectada. Como discutido anteriormente, este tipo de ataque se fundamenta nas imperfeições de implementação, especialmente no que se refere a fonte de fótons, que em um caso real pode produzir mais de um fóton com o mesmo estado por pulso.

No ambiente de simulação o comportamento da fonte de fótons foi implementado por meio da função photonSource. Esta implementa a distribuição de Poisson, discutida anteriormente, que corresponde a estatística verificada em casos práticos. Por meio dessa distribuição, é possível definir, para uma intensidade de feixe $\mu$, o número de fótons médio por pulso.

A figura 36 mostra o resultado da implementação da função de distribuição de probabilidade de $x$ ocorrências na distribuição de Poisson para uma média de intensidade de fótons $\mu$, definida pela equação:

$$
f(x)=\frac{\mu^{x} \cdot e^{-\mu}}{x}
$$

Da distribuição de Poisson é possível calcular a probabilidade de ao menos uma ocorrência, definida pela equação:

$$
P(x \geq 1)=1-p(0)=1 \frac{\mu^{0} \cdot e^{-\mu}}{0 !}=1-e^{-\mu},
$$

e mostrada na figura 37. 


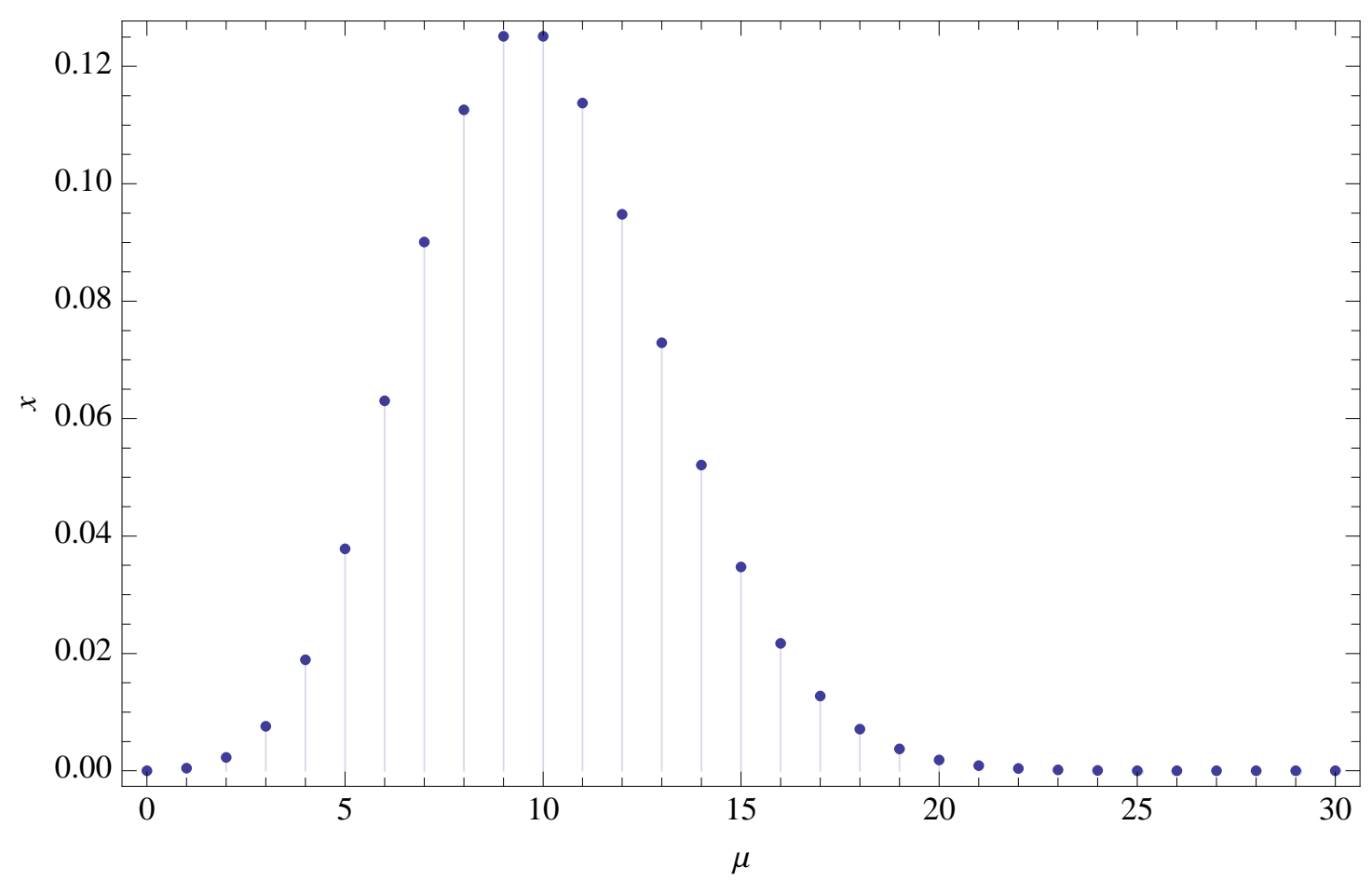

Fig. 36: Função de distribuição de probabilidade de $x$ ocorrências na distribuição de Poisson para uma média de intensidade de fótons $\mu$

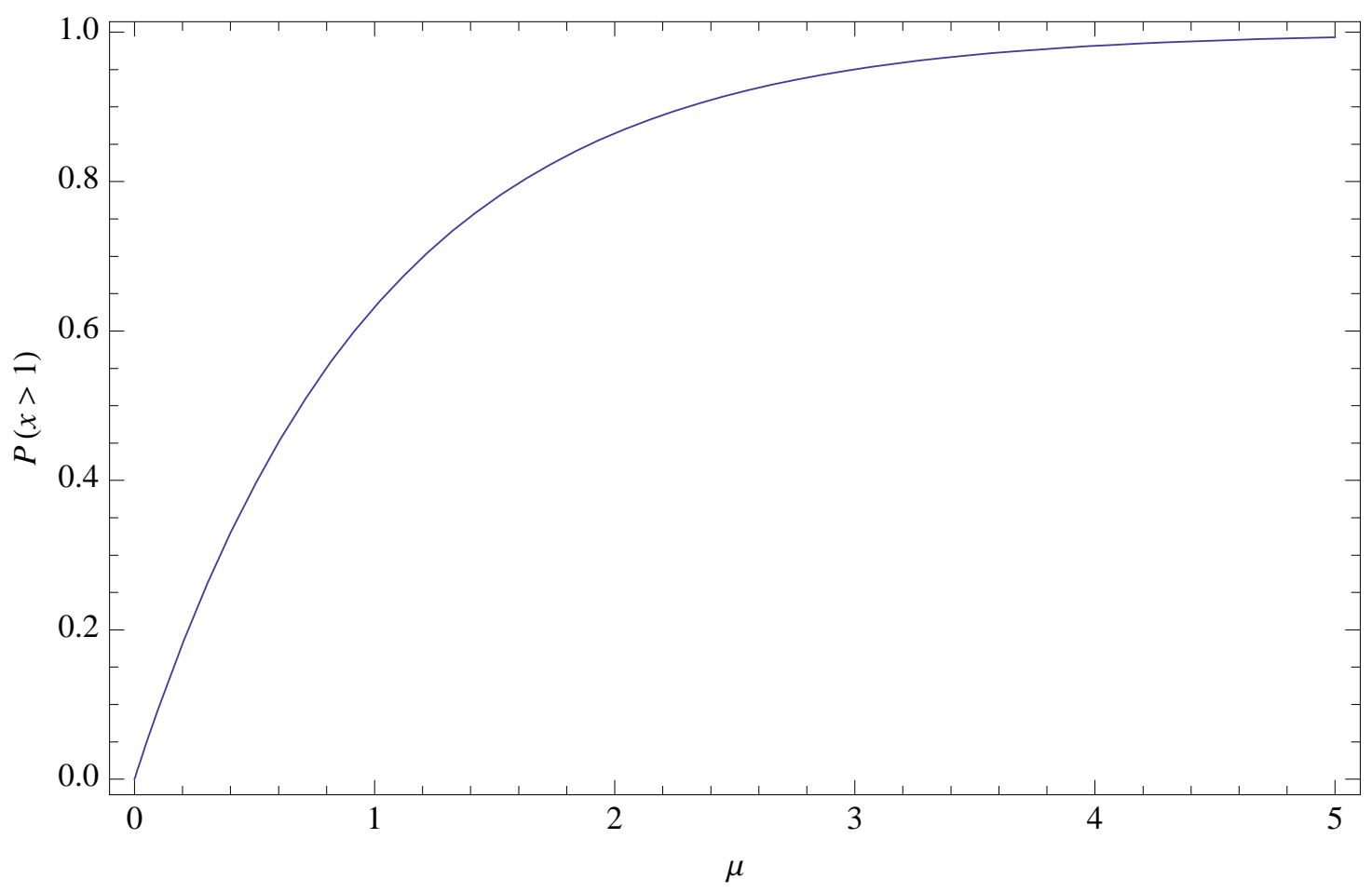

Fig. 37: Probabilidade de ao menos uma ocorrência na distribuição de Poisson em função da intensidade $\mu$ 
Com isso, considerando que a implementação da fonte de fótons tem o comportamento esperado para um caso prático, é possível estabelecer os parâmetros para a execução da etapa de amplificação de privacidade, de acordo com a teoria desenvolvida na seção 4.1.2.6. Esta etapa é representada pela execução da função de Hash, implementada pela função de simulação universalHash, que executa a ação $\left\{0,1^{n}\right\} \rightarrow\left\{0,1^{n-l-s}\right\}$, apresentada em 7.1.1. Utilizando, como anteriormente, um erro médio $e$ para o canal quântico de $\approx 5 \%$ e uma intensidade $\mu=0,12$ (este é um valor tipicamente encontrado em implementações físicas, como será visto na seção 7.2.2), pode-se calcular o valor do parâmetro $l$ por meio da equação (7.7):

$$
5 \sqrt{4+2 \sqrt{2} e+\mathrm{N} \mu(1-\mu)}+N(2 \sqrt{2}+\mu)=3,34298 \sqrt{N}+0,147421 N
$$

com $N$ sendo o tamanho da sequência inicial, i.e., a sequência estabelecida entre Alice e Bob após o anúncio de bases.

A definiçãa do parâmetro $s$ está relacionada a informação máxima que Eva possuirá após o processo de amplificação de privacidade. Para uma taxa de informação máxima igual a $10^{-3}$ bits, $s$ pode ser obtido a partir da equação (7.3):

$$
\frac{2^{s}}{\ln 2}=10^{-3} \Rightarrow s=10,4946
$$

Com isso, a partir dos valores de $l$ e $s$ é possível obter o tamanho final da chave em função de $N$ :

$$
f_{k}=n-l-s=n-3,34298 \sqrt{N}-0,147421 N-10,4946,
$$

sendo $n$ o tamanho da sequência original antes do processo de amplificação de privacidade e $N$ o tamanho da sequencia após o anúncio de bases. De (7.10) tem-se que $\bar{N} \approx r_{k} / 2$ e de (7.11) tem-se que $\bar{n} \approx r_{k} / 5$, com $r_{k}$ sendo o tamanho da sequencia original de qubits codificadas por Alice. Assim, a equação (7.18) se torna:

$$
f_{k}=-10,4946-2,36385 \sqrt{r_{k}}+0,126289 r_{k}
$$


A figura 38 mostra a relação entre o tamanho final da chave obtida após o processo de amplificação de privacidade em função do tamanho inicial da sequencia de qubits codificadas por Alice.

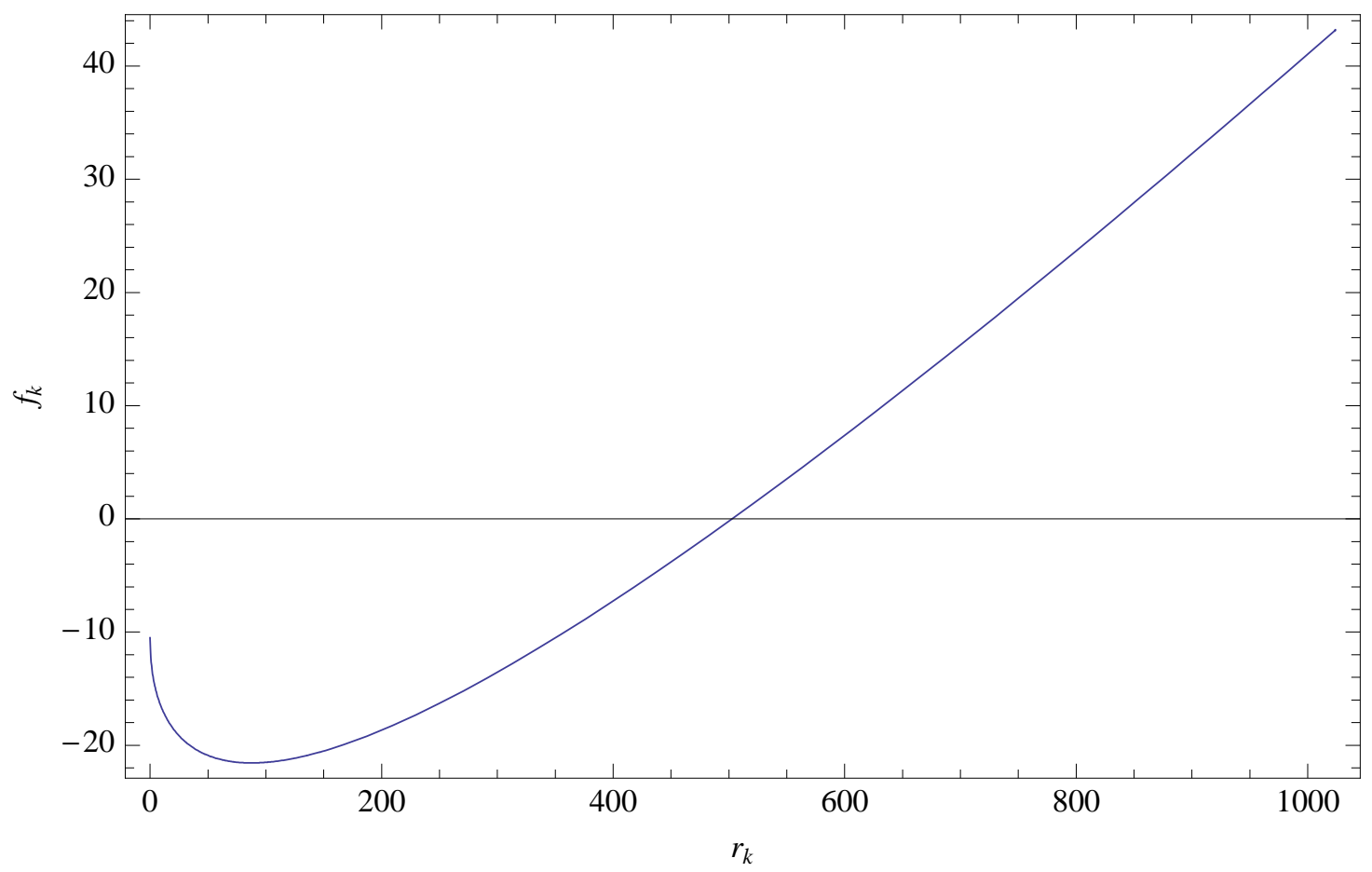

Fig. 38: Relação entre o tamanho final da chave obtida após o processo de amplificação de privacidade em função do tamanho inicial da sequencia de qubits

O tamanho da sequência de qubits $r_{k}$ codificados por Alice pode ser associado diretamente a uma taxa média de geração de qubits $\overline{Q B R}$. Com isso, a partir da equação (7.19) e com o auxílio da figura 38 , obtém-se então de forma imediata a relação de desempenho para a obtenção da chave final, i.e., a taxa de geração de chave $K G R$ :

$$
\overline{K G R}=-10,4946-2,36385 \sqrt{\overline{Q B R}}+0,126289 \overline{Q B R}[\mathrm{bits} / \mathrm{s}]
$$

Da figura 38 constata-se ainda que o tamanho inicial da sequência de qubits codificadas por Alice antes da execução do protocolo deve ser maior que um valor mínimo $r_{k}$, dado por:

$$
f_{k}>0 \Rightarrow-10,4946-2,36385 \sqrt{r_{k}}+0,126289 r_{k}>0 \Rightarrow r_{k}>502.817
$$


Obtida a relação de desempenho esperada para o protocolo desenvolvido é possível então, utilizando dados de implementações físicas reais, estimar o desempenho esperado para uma implementação real do protocolo simulado. Isso é feito nas seções seguintes.

\subsubsection{Valores de refência para comparação}

Com a intenção de aproximar ao máximo os resultados da simulação e aqueles que podem ser obtidos fisicamente com a tecnologia atual de fabricação dos dispositivos necessários para a implementação de protocolos de criptografia quântica, são avaliados os dados obtidos pelas técnicas de implementação que já tiveram sucesso demonstrado experimentalmente.

No âmbito de tecnologias comercialmente disponíveis, podem ser comparados os melhores índices de dois dispositivos: MagiQ QPN 7505 e Clavis 3000, fornecidos respectivamente por MagiQ Technologies e idQuantique. Adicionalmente, podem ser considerados os índices obtidos pela companhia QnetiQ na implementação de protocolos de criptografia quântica por espaço aberto, mesmo considerando que atualmente a tecnologia desenvolvida está disponível apenas no âmbito militar/acadêmico mas com previsão de comercialização. Os valores de referência consultados serão os valores nominais divulgados na documentação fornecida pelas companhias.

No âmbito da pesquisa acadêmica/militar, duas instituições se destacam por avanços obtidos na implementação de protocolos quânticos divulgados frequentemente. Estes são, como discutido anteriormente, o NIST ( National Institute of Standards and Technology) do Departamento de Comércio Norte Americano e a empresa BBN Technologies, que é financiada pela DARPA (Defense Advanced Research Projects Agency) do Departamento de Defesa Norte Americano. Os valores de referência utilizados serão aqueles disponibilizados nos mais recentes artigos científicos publicados relacionados a projetos de pesquisa das instituições, sendo indicados quando referenciados.

Em ambos os âmbitos de pesquisa há duas abordagens paralelas na implementação de protocolos de criptografia quântica: a execução deste por espaço aberto ou por meio físico, tipicamente fibra óptica. Como o cenário proposto de integração de DCQ na estrutura da ATN pode utilizar ambas as abordagens, são comparados os índices para os dois casos. Contudo, uma análise de viabilidade é feita somente para o caso de 
comunicação por espaço aberto.

Os índices de desempenho relevantes são, como discutido anteriormente, a taxa de codificação de qubits (Quatum Bit Rate, $Q B R$ ), taxa de erros nos qubits (Quantum Bit Error Rate, $Q B E R$ ) e taxa de geração de chave (Key Generation Rate, $K G R$ ). Em uma implementação física, três parâmetros principais influenciam esses índices de desempenho: distância máxima de transmissão $\left(D_{\max }\right)$ e a média de fótons presentes em cada pulso $(\mu)$.

Os melhores índices de desempenho encontrados para execução de protocolo quântico por fibra óptica estão disponíveis nas referências (HISKETT et al., 2006; TANG et al., 2006) e são resumidos na tabela 10 de acordo com o dispositivo ou experimento de referência.

Tab. 10: Índices de desempenho de dispositivos de DCQ por fibra óptica

\begin{tabular}{l||ccccc}
\hline Referência & QBR (Kqubits/s) & QBER $(\%)$ & KGR $($ Kbits/s) & $D_{\max }(m)$ & $\mu$ \\
\hline \hline MagicQ 7505 & $<10000$ & $\approx 1 \%$ & $<100$ & $\approx 120.000$ & 0,12 \\
Clavis 3000 & $<15000$ & $\approx 1,5 \%$ & $<150$ & $\approx 100.000$ & 0,12 \\
NIST & 1600 & $5,6 \pm 0,3$ & $\approx 51$ & 67100 & 0,2 \\
\hline
\end{tabular}

Os índices de desempenho das realizações experimentais por espaço aberto mais bem sucedidas estão disponíveis nas referências (MA et al., 2007; SCHMITTMANDERBACH et al., 2007) e resumidas na tabela 11.

Tab. 11: Índices de desempenho de dispositivos de DCQ por espaço aberto

\begin{tabular}{l||ccccc}
\hline Referência & QBR (Kqubits/s) & QBER $(\%)$ & KGR (Kbits/s) & $D_{\max }(m)$ & $\mu$ \\
\hline \hline QinetiQ & $\approx 1000$ & $\approx 5 \%$ & não divulgado & $\approx 144.000$ & 0,12 \\
NIST & $\approx 2320$ & $3,0 \pm 0,1$ & $\approx 43$ & $\approx 23.700$ & 0,2 \\
\hline
\end{tabular}

Os índices de desempenho de implementações de criptografia quântica têm evoluído fortemente nos últimos anos por meio de um crescimento constante dos investimentos realizados por diversos institutos pelo mundo, como comentado anteriormente e citado em 4.2. Dos argumentos apresentados na seção 4.2, conclui-se que atenção especial deve ser dada às tecnologias de implementação de criptografia quântica por espaço aberto, uma vez que essa abordagem permitiria a obtenção de uma rede global de DCQ. Nesse sentindo o trabalho conjunto feito pela companhia QinetiQ e o projeto SECOQC tem-se mostrado fundamental, sendo este grupo responsável pela tecnologia 
de implementação mais eficiente disponível atualmente na realização de criptografia quântica por grandes distâncias mantendo um baixo nível de ruído. O plano de trabalho do consórcio indica ainda um planejamento consistente para a evolução da tecnologia atual.

Com os dados obtidos para o desempenho da implementação de protocolos quânticos por espaço aberto realizada pelo grupo QinetiQ/SECOQC, é possível avaliar o desempenho esperado para a implementação da proposta desenvolvida neste trabalho e simulada anteriormente. Devido ao caráter estratégico do tema, os valores para a taxa de geração de chave $(K G R)$ no mecanismo desenvolvido pelo grupo QinetiQ/SECOQC não é divulgado, não permitindo uma comparação direta com o valor que pode ser encontrado para a abordagem desenvolvida aqui. Contudo, uma comparação indireta pode ser feita com os valores divulgados por outras abordagens de implementação.

Os cálculos da seção anterior foram baseados nos parâmetros de erro e intensidade média de fótons por pulso referentes a realização da QinetiQ, mostrados na tabela 11. Com isso, a estimativa de desempenho para o protocolo desenvolvido ao longo desse trabalho é obtida a partir da equação:

$$
\overline{K G R}=-10,4946-2,36385 \sqrt{\overline{Q B R}}+0,126289 \overline{Q B R},
$$

e com os dados da tabela 11 tem-se:

$$
\overline{K G R} \approx-10,4946-2,36385 \sqrt{1000}+0,126289 \cdot 1000=\mathbf{4 1 , 0 4 3 4} \text { Kbits } / \mathbf{s}
$$

A comparação da taxa de geração de chaves $(K G R)$ encontrada para a proposta desenvolvida ao longo das seções anteriores, obtida utilizando como referência os valores nominais dos dispositivos desenvolvidos pelo grupo QinetiQ/SECOQC, e aquelas mostradas nas tabelas 10 e 11 evidenciam um ganho de eficiência. Contudo, nesta comparação devem ser considerados outros detalhes relevantes, como a natureza de implementação, por fibra óptica ou por espaço aberto, distância máxima, dentre outros. Assim, a utilização da abordagem desenvolvida neste trabalho em um ambiente prático será avaliada independentemente, o que é feito a seguir. 


\subsection{Protocolo BB84 em operação conjunta com a ATN}

Como apresentado na seção 5.1, a recomendação e as implementações atuais da ATN segundo o documento ICAO SARPs (ICAO, 2002), são fundamentadas principalmente nas redes de comunicações móveis: Mode S (Mode Select), VDL 2/4 (VHF Digital Link Mode 2/4) e UAT (Universal Access Transceivers). Essas são tecnologias de comunicação por espectro de rádio frequência especialmente desenvolvidas para a comunicação digital na aeronáutica. Uma descrição detalhada destas pode ser encontrada em (ICAO, 2002), sendo suas principais características resumidas na figura 39. Os requisitos de confiabilidade e disponibilidade para essas tecnologias tem sido alvo de diversas pesquisas nas últimas décadas, sendo que todas estas tecnologias estão em operação em diversos cenários reais (ICAO, 2002). Independentemente da rede de comunicação utilizada, a troca de informação é realizada segundo o modelo OSI e por isso totalmente adaptada a implementação dos algoritmos criptográficos atuais. A implementação sugerida no documento ICAO SARPs (ICAO, 2002) consiste na utilização do algoritmo RSA para estabelecimento de uma chave de sessão e encriptação utilizando o algoritmo simétrico de cifra de bloco AES.

O protocolo de criptografia quântica desenvolvido neste trabalho consiste em uma alternativa para esse sistema. A integração deste ao cenário atual é possível, do ponto de vista do canal de comunicação clássico, com nenhuma ou pequena adaptação. Isso por que o protocolo BB84 consiste num mecanismo de geração de chave secreta tal como ocorre com algoritmo RSA, podendo ser a chave obtida por esse método utilizada tal como acontece no caso atual. Contudo, um aspecto adicional deve ser considerado a partir da discussão promovida no capítulo 3, que mostra que não somente o RSA pode ser vulnerável como também protocolos simétricos poderiam ser corrompido na presença de tecnologia de processamento de informação avançada. Como alternativa tem-se a utilização da cifra de uso-único (one-time-pad) como mecanismo de cifragem.

Na utilização de algoritmos baseados em complexidade computacional como o RSA em conjunto com um algoritmos simétricos de cifra de bloco, a taxa de geração de chave não representa um fator crítico, uma vez que uma pequena chave é utilizada para cifrar uma grande quantidade de informação. Assim, mesmo uma baixa taxa de produção de chave permite uma grande taxa de transmissão de informação encriptada. Contudo, 
no caso da utilização da cifra de uso único, a taxa com que se pode produzir a chave é fundamental para a taxa máxima de comunicação que se consegue estabelecer, uma vez que, como visto na seção, a cifra de uso único exige uma chave do tamanho da mensagem a ser transmitida. Adicionalmente, neste caso não é possível reutilizar as chave de sessão.

Com isso, a viabilidade da utilização do protocolo de criptografia quântica desenvolvido anteriormente está condicionada ao atendimento de taxas mínimas de geração de chave. Essa taxa deve ser, no caso da utilização da cifra de uso único, equivalente a taxa de transferência requerida pelo canal de dados.

Assim, é possível comparar a estimativa de desempenho para a geração de chave obtida para a implementação física do protocolo desenvolvido com a taxa de comunicação dos principais canais de comunicação utilizados na recomendação da ATN. A figura 39 mostra uma tabela extraída das especificações da ICAO (ICAO, 2002) que relaciona, dentre outras informações, a taxa de transferência verificadas para as principais tecnologias de comunicação de dados recomendadas para a implementação da ATN.

O resultado obtido para uma estimativa de desempenho para uma implementação física do protocolo BB84 em conjunto com as técnicas desenvolvidas neste trabalho, mostrado na equação (7.23), é, quando comparado ao alto desempenho requisitado em algumas redes de telecomunicações, relativamente baixo. Contudo, quando comparado às taxas de transferências verificadas nos principais canais de comunicação utilizados na aeronáutica, o desempenho do protocolo de criptografia quântica passa ser considerável. O índice desempenho obtido de 41,0434 Kbits/s é suficientemente maior que a taxa de transferência média verificada na tecnologia de comunicação VDL Mode 4, que não excede 38, 4 Kbits/s. Isto implica que, considerando que seja possível obter os índices encontrados na implentação experimental realizada pela QinetiQ para o protocolo BB84 em ambientes práticos de operação, a abordagem utilizada permitiria a manutenção da segurança de canais de dados da ATN baseados em VDL Mode 4 utilizando um protocolo de criptografia quântica.

As tecnologias UAT e Mode S possuem taxa de transferência nominais duas ordens de grandeza maior que aquelas obtidas para o protocolo BB84. Esses são valores nominais e não correspondem às taxas de transferências verificadas na operação dos 


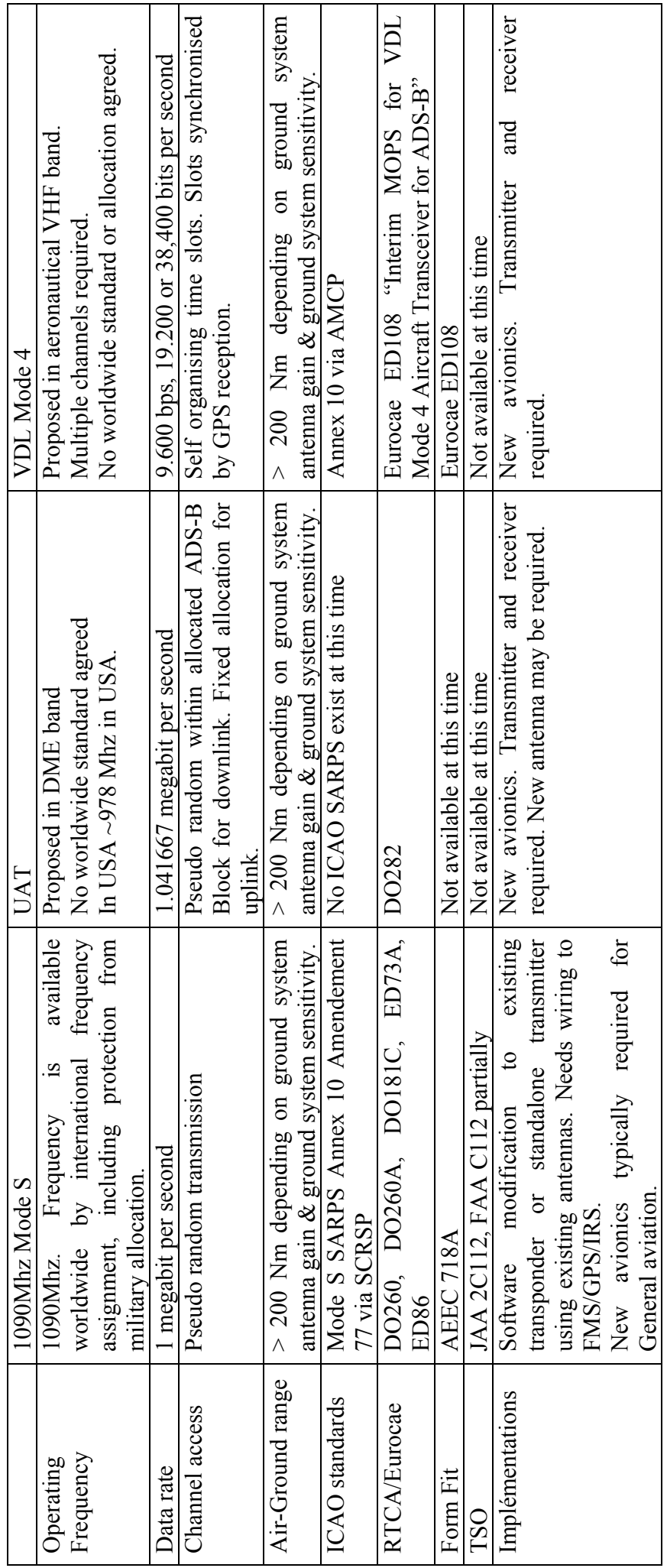

Fig. 39: Referências técnicas para tecnologias de comunicação utilizadas na ATN a partir de acordo com a especificação ICAO (ICAO, 2002) 
atuais sistemas de comunicação utilizados na aeronáutica (ICAO, 2002). Entretanto, a perspectiva apontada pelas entidades reguladoras deste ambiente é de que o tráfego de informação na ATN pode facilmente alcançar taxas de transferência de dados próximas daquelas disponíveis nas tecnologias UAT e Mode S (ICAO, 2002).

Esse fato não implica necessariamente que a comunicação de dados por canais utilizando estas tecnologias não seria passível de proteção por meio do protocolo BB84. A limitação do desempenho obtido diante das taxas de transferências verificadas nessas tecnologias indica apenas que a tecnologia atual de implementação por espaços abertos, no cenário que foi proposto, não se encontra madura o suficiente para ser utilizada. Contudo, considerando a rápida evolução verificada nos últimos anos na implementação de protocolos de criptografia quântica por espaços abertos e os índices de desempenho que já são obtidos para implementações em fibra óptica, como aqueles apresentados em 4.2.1, é plausível se esperar que estes índices possam ser alcançados em curto prazo. Com a manutenção da taxa de evolução verificada nos últimos 10 anos, uma extrapolação da evolução dos índices de desempenho das técnicas de implementação por espaço aberto indica que os índices de desempenho requeridos pelas tecnologias UAT e Mode $\mathrm{S}$ podem ser alcançados período de até 5 anos.

\subsection{Considerações finais do capítulo}

Os resultados obtidos na simulação do protocolo BB84 e de desenvolvimentos adicionais mostraram que a abordagem de implementação computacional escolhida foi adequada. Isso pôde ser verificado por meio da obtenção de uma estatística para a execução do protocolo BB84 condizente com a teoria desenvolvida no capítulo 4. Adicionalmente, a implementação e simulação dos procedimentos de reconciliação de informação e amplificação de privacidade permitiu verificar o impacto destes sobre a operação do protocolo BB84.

Este impacto foi medido por meio da obtenção do desempenho na geração de uma chave de sessão verificado no ambiente de simulação. A utilização de dados das principais implementações físicas de protocolos de criptografia quântica existentes permitiu estimar como o índice de desempenho obtido se traduziria em uma implementação física 
da proposta desenvolvida nos cenários típicos de comunicação verificados nos cenários propostos de integração de uma IDCQ à ATN.

Os índices de desempenho obtidos indicam que a tecnologia atual para a implementação de protocolos quânticos satisfaz o requisito de desempenho exigido por apenas uma das principais tecnologias de comunicação especificadas na ATN (VDL Mode 4). Contudo, a demonstração do funcionamento do protocolo BB84 e das técnicas propostas indica que, com o desenvolvimento das técnicas de implementação disponíveis, essa abordagem poderá ser utilizada em um futuro próximo como mecanismo de manutenção de segurança da ATN.

Entretanto, a abordagem desenvolvida aqui avaliou apenas um dos aspectos críticos relacionados a operação da ATN. Este trabalho mostrou que o atendimento de uma taxa de transferência mínima pode ser alcançado por um protocolo de criptografia quântica atualmente ou em um futuro próximo de acordo com a tecnologia de comunicação utilizada. Contudo, outros aspectos relacionados aos requisitos de tempo real, como a disponibilidade verificada na implementação da infra-estrutrura sugerida, a confiabilidade dos dispositivos utilizados, dentre outros, não foram avaliados.

Assim, é possível concluir dos resultados obtidos com o ambiente de simulação do protocolo BB84 operando na estrutura da ATN que este já possui maturidade teórica para ser utilizado como mecanismo de segurança da informação nesse cenário. Contudo, percebe-se que a maturidade das técnicas de implementação ainda necessita de mais desenvolvimento e uma avaliação mais extensa para sua implementação efetiva em casos práticos, sendo este trabalho um passo nessa direção. 


\section{Considerações Finais}

\subsection{Conclusões}

A revisão dos fundamentos matemáticos das principais técnicas de criptologia clássica utilizadas atualmente, apresentada no capítulo 2, e os resultados exibidos pelo desenvolvimento de uma teoria de computação quântica, apresentados no capítulo 3, evidenciaram que alguns dos conceitos utilizados como fundamentos da criptologia clássica necessitam ser revistos no caso da computação quântica se tornar tecnologicamente viável. Tal conclusão decorre do ganho de eficiência que computadores quânticos demonstram no tratamento de alguns problemas computacionais que não possuem tratamento eficiente por computadores clássicos. O desenvolvimento do algoritmo de busca de ordem apresentado na seção 3.2.3 e, a partir deste, o desenvolvimento do algoritmo de fatoração apresentado na seção 3.2.4 e sua aplicação a solução do algoritmo RSA, mostraram que o problema computacional utilizado como mecanismo de segurança deste algoritmo pode ser eficientemente tratado por um computador quântico, invalidando a segurança do algoritmo criptográfico assimétrico mais amplamente utilizado atualmente.

Os requisitos de capacidade de processamento para um computador quântico exigidos para o corrompimento do algoritmo RSA em casos práticos fizeram com que a fragilidade dos métodos criptográficos atuais diante da computação quântica não representasse um fator crítico até o momento. Isso porque o corrompimento de chaves RSA com o tamanho médio utilizado hoje necessitaria da implementação de um computador quântico com capacidade muito além daquela que, em princípio, seria possível obter com a tecnologia disponível no momento. Contudo, os resultados recentes apresentados na seção 3.3, referentes a implementação de computação quântica experimental 
pelo setor privado, indicam que a obtenção prática de computação quântica disponível comercialmente e com capacidade suficiente para corromper as chaves RSA atuais poderia ocorrer em um prazo inferior ao que a tecnologia disponível atualmente sugeriria. Se verdadeiros, tais resultados tornariam alguns dos principais algoritmos criptográficos clássicos inviáveis em curto período.

A preocupação relacionada a inviabilização dos métodos atuais de criptografia levou a investigação das alternativas disponíveis para as técnicas atuais, apresentada no capítulo 4. A despeito da possibilidade da utilização de problemas computacionais que ainda não possuem tratamento eficiente pela computação quântica como fundamento para uma nova classe de protocolos criptográficos, a chamada criptografia pós-quântica, a escolha desta pesquisa foi a exploração do novo paradigma representado pela utilização de sistemas quânticos na obtenção de protocolos criptográficos, conhecidos de forma geral como criptografia quântica.

A investigação dos fundamentos teóricos da criptografia quântica, realizada na seção 4.1, e o levantamento dos protocolos criptográficos quânticos disponíveis, realizado na seção 4.1.1, levaram a escolha do protocolo BB84 como a alternativa que melhor se adequa a utilização em casos práticos. Isso se deveu a dois fatores principais: a existência de provas formais de segurança para o protocolo BB84 e ao avançado desenvolvimento das técnicas de implementação física do protocolo como discutidos nas seções 4.1 .5 e 4.2 , respectivamente.

Por meio da análise do funcionamento teórico do protocolo BB84 em um cenário de comunicação entre duas partes, mostrada na seção 4.1.2, foi possível identificar alguns de seus pontos de vulnerabilidade. A partir dessa análise foram listados alguns ataques que poderiam se beneficiar dos pontos vulneráveis para corromper o protocolo, tal como mostrado na seção 4.1.3. A medida de contenção encontrada na literatura consiste na inclusão de duas etapas adicionais na operação do protocolo: o procedimento de reconciliação de informação e o procedimento de amplificação de privacidade.

A partir do conhecimento encontrado na literatura, foram desenvolvidos dois métodos para a realização dos procedimentos de reconciliação de informação e amplificação de privacidade. Para a reconciliação de informação foi desenvolvido o algoritmo Cascade Modificado. Este representa uma contribuição deste trabalho na otimização 
do algoritmo Cascade proposto na literatura. Na etapa de amplificação de privacidade foi utilizada uma abordagem equivalente a encontrada na literatura, representada pelo uso de funções de Hash da classe das funções universais-2. A análise teórica do funcionamento deste mostrou que a inclusão destes procedimentos inviabiliza os ataques propostos na seção 4.1.3.

O levantamento do estágio de desenvolvimento das técnicas de implementação física do protocolo BB84 mostrou um campo de intensa pesquisa e rápidas mudanças. Os índices de desempenho na taxa de transmissão de informação exibidos pelas melhores técnicas mostraram que estas já alcançam ou têm perspectivas de alcançar índices de desempenho da ordem de grandeza verificada em algumas redes de telecomunicação em operação.

Nesse sentindo, a escolha do ambiente de telecomunicações aeronáuticas como caso de estudo se mostrou bastante propícia, uma vez que os índices de desempenho verificados nas diferentes tecnologias de comunicação utilizadas nesse caso serem relativamente baixos quando comparados à outros ambientes. Esse aspecto tornaria a possível utilização de protocolos de criptografia quântica nesse ambiente viável em um menor prazo.

A análise quatitativa do desempenho esperado a utilização do protocolo BB84 e os procedimentos teóricos desenvolvidos na seção 4.1.2.5 e 4.1.2.6 foi feita por meio da proposição de alguns cenários de integração do protocolo quântico à infra-estrutura atual e a simulação das propostas teóricas para o protocolo BB84.

A avaliação da atual infra-estrutura da Aeronautical Telecommunication Network (ATN) e a implementação de segurança da informação utilizada por esta permitiu avaliar possíveis cenários de integração para o protocolo BB84. A conclusão desta avaliação é representada pelo cenário em que se tem o protocolo BB84 operando com transmissores baseados em uma malha de satélites e receptores embarcados em aeronaves ou presentes em estações de solo.

A avaliação quantitativa do cenário proposto foi feita por meio da obtenção de um ambiente de simulação para o protocolo BB84 e para os desenvolvimentos teóricos propostos. A simulação do aspecto propabilístico, derivado da natureza quântica do protocolo BB84, foi beneficiada pela a utilização do serviço de geração de números aleatórios 
baseados em sistema quântico Quantum Random Bit Generator (QRBG). Tal benefício foi verificado nas medidas estatísticas realizadas para a operação simulada do protocolo BB84 mostrada no capítulo 7. Os resultados evidenciam uma estatística em total acordo com aquela esperada teoricamente.

A simulação dos desenvolvimentos teóricos relacionados às etapas de reconciliação de informação e amplificação de privacidade pôde ser feita a partir disso. Os resultados da aplicação do algoritmo Cascade Modificado, apresentados na seção 4.1.2.5, mostram que a proposta é eficiente na correção de erro com baixa divulgação de informação. Na seção 4.1.2.6, foi mostrado como implementar o procedimento de amplificação de privacidade a partir da implementação de uma função de hash. A utilização da mesma função aleatória utilizada para definir o colapso de estados como mecanismo de obtenção da função de hash utilizada se mostrou válida e a teoria desenvolvida para este procedimento foi aplicada com sucesso.

Como conclusão do desenvolvimento do ambiente de simulação, foi possível obter o índice de desempenho esperado para a operação do protocolo BB84 e dos desenvolvimentos teóricos realizados em uma operação prática, tal como o cenário de integração com a ATN proposto na seção 7.1. A comparação do índice de desempenho encontrado para simulação e aquele esperado em uma implementação física do cenário proposto foi feito por meio da utilização dos índices de desempenho para as melhores implementações físicas para o protocolos de criptografia quântica disponíveis. O resultado encontrado consiste em uma taxa de transmissão da ordem de 40 Kbits por segundo. Apesar de não haver dados disponíveis para uma comparação direta equivalente, comparações indiretas entre a abordagem desenvolvida no trabalho e aquelas disponíveis indicam ganho de eficiência. Tal fato evidencia que a proposta de modificação do algoritmo Cascade pôde se traduzir em ganho de eficiência no processo de correção de erros.

A comparação do desempenho encontrado com aquele verificado pela implementação atual da ATN foi feita na seção 7.1. Por meio das referências de desempenho verificadas nos diferentes canais de comunicação, verificou-se que o desempenho encontrado para uma implementação física da proposta atual atende aos requisitos de desempenho de apenas uma das tecnologias utilizadas atualmente na ATN, a rede VDL Mode 4. 
Contudo, verificando a taxa de evolução das técnicas de implementação de protocolos quânticos nos últimos anos, é possível inferir que o índice de desempenho obtido pode ser melhorado em até duas ordem de grandeza nos próximos 5 anos. Isso tornaria a utilização da abordagem desenvolvida neste trabalho viável para todas as tecnologias de comunicação utilizadas atualmente.

Por fim, o desenvolvimento deste trabalho mostrou que protocolos de criptografia quântica podem ser considerados como mecanismos candidatos para a substituição de técnicas de criptografia clássica em redes de telecomunicações práticas. A avaliação de cenários de integração, a obtenção de um ambiente de simulação e sua avaliação no caso prático da ATN mostrou que o protocolo BB84 atende ou atenderá em curto prazo os requisitos de desempenho exigidos nesse ambiente. Contudo, este trabalho representa um passo inicial no desenvolvimento de uma avaliação mais completa para a implementação de protocolos de criptografia quântica em redes de missão crítica, incluindo a avaliação de requisitos de disponibilidade, confiabilidade, dentre outros relevantes nesses tipos operação. Isso representaria o desenvolvimento de um ambiente de simulação mais completo que pudesse considerar dados de tráfego real, múltiplos agentes e variáveis realistas de ambiente de operação. Tal objetivo constitui a principal diretriz de continuação desta pesquisa. Com isso, este trabalho abre uma boa perspectiva para novos desenvolvimentos na obtenção de um mecanismo eficiente para a manutenção da segurança da informação em sistemas críticos 


\section{Referências}

ASPELMEYER, M. et al. Long-distance quantum communication with entangled photons using satellites. IEEE Journal of Quantum Electronics, v. 9, n. 6, p. 1541-1551, 2003.

BENNETT, C.; BRASSARD, G. Quantum cryptography: Public key distribution and coin tossing. IEEE International Conference on Computers, Systems and Signal Processing, v. 1, p. 175-179, 1984.

BENNETT, C. H. Quantum cryptograhy using any two nonorthogonal states. Phys. Rev. Lett., v. 68, p. 3121-3124, 1992.

BENNETT, C. H. et al. Experimental quantum cryptography. Lecture Notes in Computer Science, v. 473, p. 253, 1991.

BENNETT, C. H. et al. Experimental quantum cryptography. Journal of Cryptology, v. 5, n. 1, p. 3-28, 1992. Disponível em: $<\mathrm{http}: / / \mathrm{www}$. springerlink.com/content/v2v212561m157017>.

BENNETT, C. H. et al. Generalized privacy amplification. IEEE Trans Information Theory, v. 41, p. 1915-1923, 1995.

BENNETT, C. H.; BRASSARD, G.; ROBERT, J.-M. Privacy amplification by public discussion. SIAM J. Comput., Society for Industrial and Applied Mathematics, Philadelphia, PA, USA, v. 17, n. 2, p. 210-229, 1988. ISSN 0097-5397.

BOUWMEESTER, D. The Physics of Quantum Information. [S.1.]: Springer-Verlag, 2000 .

BRASSARD, G.; SALVAIL, L. Key reconciliation by public discussion. Lecture Notes in Computer Science, v. 765, p. 410-423, 1994.

BRYLINSKI, R. K.; CHEN, G. Classical and Quantum Computation. [S.1.]: American Mathematical Society, 2002.

BUTTLER, W. T. et al. Practical free-space quantum key distribution over $1 \mathrm{~km}$. Phys. Rev. Lett., American Physical Society, v. 81, n. 15, p. 3283-3286, Oct 1998.

COHEN-TANNOUDJI, C.; DUI, B.; LALOE, F. Quantum Mechanics. [S.1.]: John Wiley \& Sons, 1978.

CORMEN, T. H. et al. Introduction to Algorithms. 4th. ed. Cambridge, Massachusetts: MIT Press/McGraw Hill, 2003. 
COSTA, C. H. A.; CAMARGO JÚNIOR, J. B. New proposal for the classical channel authentication problem in QKD protocols.

COSTA, C. H. A.; CAMARGO JÚNIOR, J. B. Segurança da informação em telecomunicações aeronáuticas: discussão da viabilidade e benefícios da utilização de criptografia quântica. In: Anais V Simpósio de Transportes Aéreos. Brasília, Brasil: Editora Universidade de Brasília, 2006. v. 1.

D-WAVE. D-Wave RoadMap. 2008. Disponível em: <http://www.dwavesys.com/>.

DENNING, D. E. R. Cryptography and Data Security. Cambridge, Massachusetts: Addison-Wesley Pub, 1982.

DEUTSCH, D. Quantum theory, the church-turing principle and the universal quantum computer. Proc. R. Soc., London, v. 400, p. 97-117, 1985.

DIFFIE, W.; HELLMAN, M. E. New directions in cryptography. IEEE Transactions on Information Theory, IT-22, n. 6, p. 644-654, 1976.

DIFFIE, W.; LANDAU, S. Privacy on the line: the politics of wiretetapping and encryption. Cambridge, Massachusetts: MIT Press, 1998.

EINSTEIN, A.; PODOLSKY, B.; ROSEN, N. Can quantum-mechanical description of physical reality be considered complete? Physical Review, p. 777, 1935.

ELBERT, B. R. The Satellite Communication Applications Handbook. Massachusetts: Artech House, 2002.

ELGAMAL, T. A public key cryptosystem and a signature scheme based on discrete logarithms. IEEE Trans Information Theory, v. 31, p. 469-472, 1985.

ELLIOT, C. Building The Quantum Networks. 2002. Disponível em: $<$ http://www.bbn.com>.

GISIN, N. et al. Quantum cryptography. Rev. Mod. Phys, v. 74, p. 145, 2002.

GLAUBER, R. J. Photon correlations. Phys. Rev. Lett., v. 10, p. 84-86, 1963.

GOBBY, C.; YUAN, Z. L.; SHIELDS, A. J. Quantum key distribution over $122 \mathrm{~km}$ of standard telecom fiber. Applied Physics Letters, v. 84, p. 3762-3764, 2004. Disponível em: <doi:10.1063/1.1738173>.

GOBBY, C.; YUAN, Z. L.; SHIELDS, A. J. Quantum Information and Computation. 2005. Disponível em: <http://www.citebase.org/abstract?id=oai:arXiv.org:quant$\mathrm{ph} / 0602125>$.

GOBBY, C.; YUAN, Z. L.; SHIELDS, A. J. Quantum Cryptography. 2007. Disponível em: <http://www.citebase.org/abstract?id=oai:arXiv.org:quant-ph/0603207>.

GOTTESMAN, D.; LO, H.-K. Proof of security of quantum key distribution with two-way classical communications. IEEE Transactions on Information Theory, v. 49, p. 457, 2003. 
GROVER, L. K. Quantum computers can search arbitra-

rily large databases by a single query. 1997. Disponível em:

$<$ http://www.citebase.org/abstract?id=oai:arXiv.org:quant-ph/9706005>.

HISKETT, P. A. et al. Long-distance quantum key distribution in optical fibre. New Journal of Physics, v. 8, n. 9, p. 193, 2006. Disponível em: <http://stacks.iop.org/13672630/8/193>.

HUGHES, R. J. et al. Quantum cryptography for secure satellite communications. 2000 IEEE Aerospace Conference Proceedings, v. 1, p. 191-200, 2000.

ICAO. ATN Standards and Recommended Practices. [S.1.]: International Civil Aviation Organization, 2002.

JACOBS, B. C.; PITTMAN, T. B.; FRANSON, J. D. Quantum relays and noise suppression using linear optics. Phys. Rev. A, American Physical Society, v. 66, n. 5, p. 052307, Nov 2002.

KIM, T. et al. Complete physical simulation of the entangling-probe attack on the bennett-brassard 1984 protocol. Physical Review A (Atomic, Molecular, and Optical Physics), APS, v. 75, n. 4, p. 042327, 2007.

KURTSIEFER, C. et al. A step towards global key distribution. Nature, v. 419, n. 3, p. 450, 2002.

LAMPORT, L. Constructing digital signatures from a one-way function. Technical Report CSL-98, SRI International, 1979.

LEWIS, T. G.; PAYNE, W. H. Generalized feedback shift register pseudorandom number algorithm. J. ACM, ACM Press, New York, NY, USA, v. 20, n. 3, p. 456-468, 1973.

LO, H. K.; POPESCU, S.; SPILLER, T. Introduction to Quantum Computation and Information. [S.1.]: World Scientific Publishing Company, 2001.

LOMONACO, S. J. Quantum Computation. [S.1.]: American Mathematical Society, 2002.

MA, L. et al. Experimental demonstration of an active quantum key distribution network with over gbps clock syschronization. IEEE Communications Letters, v. 11, n. 12, 2007.

MARKS, P. Quantum cryptography to protect swiss election. New Scientist, 2007.

MAYERS, D. Unconditional security in quantum cryptography. $J A C M$, v. 3, p. 35, 1998. Disponível em: <http://www.citebase.org/abstract?id=oai:arXiv.org:quant$\mathrm{ph} / 9802025>$.

MCMATH, J. Aeronautical Telecommunications Network (ATN): Security, Key Management and Distribution. [S.1.]: AEEC Data Link Users Forum and ESC/GAD, 2003. 
MENEZES, A. J.; OORSCHOT, P. C. van; VANSTONE, S. A. Handbook of Applied Cryptography. [S.1.]: CRC Press, 2001.

MEYER, U.; WETZEL, S. On the impact of gsm encryption and man-in-the-middle attacks on the security of interoperating gsm/umts networks. Personal, Indoor and Mobile Radio Communications, 2004. PIMRC 2004. 15th IEEE International Symposium on, v. 4, p. 2876-2883, 2004.

MINKEL, J. R. First "commercial"quantum computer solves sudoku puzzles. Scientific American, 2007. Disponível em: <http://www.sciam.com/article.cfm?id=firstcommercial-quantum-computer $>$.

MOORE, S. K. Prototype of a commercial quantum computer demonstrated. IEEE Spectrum, 2007. Disponível em: $<$ http://www.spectrum.ieee.org/feb07/comments/1710>.

NAOR, M.; YUNG, M. Universal one-way hash functions and their cryptographic applications. In: Proceedings of the Twenty First Annual ACM Symposium on Theory of Computing. (May 15-17 1989: Seattle, WA, USA). New York, NY 10036, USA: ACM Press, 1989. p. 33-43.

NEWS@NATURE. Quantum leap of faith. Nature, v. 446, p. 245-245, 2007.

NIELSEN, M. A.; CHUANG, I. L. Quantum computation and Quantum Information. Cambridge, Massachusetts: Cambridge University Press, 2000.

POPPE, A. et al. Practical quantum key distribution with polarizationentangled photons. Optics Express, v. 12, p. 3865, 2004. Disponível em: $<$ http://www.citebase.org/abstract?id=oai:arXiv.org:quant-ph/0404115>.

REGEV, O. Quantum computation and lattice problems. Foundations of Computer Science, 2002. Proceedings. The 43rd Annual IEEE Symposium on, p. 520-529, 2002.

RIGUIDEL, M. QuantumCrypt - Enhancement of AGT Communications Security Using Quantum Cryptography. [S.1.]: EUROCONTROL, 2004.

RITTER, T. The efficient generation of cryptographic confusion sequences. Cryptologia, v. 15, n. 2, p. 81-139, 1991.

SCHMITT-MANDERBACH, T. et al. Experimental demonstration of free-space decoy-state quantum key distribution over $144 \mathrm{~km}$. Lasers and Electro-Optics, 2007 and the International Quantum Electronics Conference. CLEOE-IQEC 2007. European Conference on, 2007.

SECOQC. SECOQC Project. 2007.

SHANNON, C. Communication theory of secrecy systems. The Bell System Technical Journal, v. 28, n. 4, p. 656-715, 1949.

SHOR, P. W. Polynomial-time algorithms for prime factorization and discrete logarithms on a quantum computer. SIAM J.Sci.Statist.Comput., v. 26, p. 1484, 1997. Disponível em: <http://www.citebase.org/abstract?id=oai:arXiv.org:quant$\mathrm{ph} / 9508027>$. 
SHOR, P. W.; PRESKILL, J. Simple proof of security of the bb84 quantum key distribution protocol. Phys. Rev. Lett., American Physical Society, v. 85, n. 2, p. 441-444, Jul 2000.

STIPCEVIC, M.; ROGINA, B. M. Quantum random number generator. 2006. Disponível em: <http://www.citebase.org/abstract?id=oai:arXiv.org:quant$\mathrm{ph} / 0609043>$.

STIPCEVIC, M.; ROGINA, B. M. Quantum random number generator based on photonic emission in semiconductors. Review of Scientific Instruments, AIP, v. 78, n. 4, p. 045104, 2007. Disponível em: <http://link.aip.org/link/?RSI/78/045104/1>.

TANG, X. et al. Demonstration of an active quantum key distribution network. Proc. SPIE, v. 6305, n. 630506, 2006.

WANG, J.; ZHANG, Q.; TANG, C. Quantum signature scheme with message recovery. Advanced Communication Technology, 2006. ICACT 2006. The 8th International Conference, v. 2, p. 4 pp.-, 2006.

WEGMAN, M. N.; CARTER, L. New hash functions and their use in authentication and set equality. J. Comput. Syst. Sci., v. 22, n. 3, p. 265-279, 1981.

ZENG, G.; KEITEL, C. H. Arbitrated quantum-signature scheme. Phys. Rev. A, American Physical Society, v. 65, n. 4, p. 042312, Apr 2002. 


\section{APÊNDICE A - Requisitos matemáticos}

\section{A.1 Definições}

Maior divisor comum: Assumindo dois inteiros positivos $a, b \in \mathbb{Z}^{+}$, o maior divisor comum destes é identificado como $m d c(a, b)$ e definido como o maior número inteiro $c$ que divide ambos $a$ e $b$. Ex.: $\operatorname{md} c(16,20)=5$.

Número primo: um inteiro $a \geq 2$ é dito primo se este pode ser divido somente por 1 ou ele mesmo, $a$, sem resto.

Primos relativos: Inteiros $a$ e $b$ são ditos primos relativos se $m d c(a, b)=1$.

Congruência: equações que tem dois lados iguais no sentido módulo $N$ são chamada congruentes e denotadas por $a \equiv b(\bmod N)$

Multiplicativo inverso: Sejam $a$ e $N$ primos relativos $(\operatorname{mdc}(a, N)=1)$ e assumindo-se que para $0<b \leq N-1,(a \cdot b) \bmod N=1$ então $b$ é chamado de multiplicativo inverso de $a$ no sentido módulo $N$.

Função de Euler: Para um inteiro positivo arbitrário $N$, a função de Euler correspondente $\varphi(N)$ resulta no número de primos relativos de $N$ no intervalo de $1,2, \ldots, N$. Ex.: $\varphi(1)=1, \varphi(10)=4$.

\section{A.2 Algoritmo de Euclides}

$\mathrm{O}$ algoritmo de Euclides tem como objetivo encontrar o $m d c(a, b)$ e pode ser detalhado da seguinte forma. 
1.Dividindo-se $a$ por $b$ obtém-se o quociente $q_{1}$ e um resto diferente de zero $r_{1}$ :

$$
a=q_{1} b+r_{1}, 0<r_{1}<b .
$$

2.b é divido por $r_{1}$, i.e. $b$ assume o papel de $a$ e $r_{1}$ que de resulta em $q_{2}$ e $r_{2}$ diferente de zero:

$$
b=q_{2} r_{1}+r_{2}, 0<r_{2}<r_{1}
$$

3.Continua-se o algoritmo até que o resto atual mantenha-se diferente de zero:

$$
r_{k}=q_{k+2} r_{k+1}+r_{k+2}, 0<r_{k+2}<r_{k+1} .
$$

4. Quando a divisão retorna $r_{l}=0$ sabe-se que $r_{l-2}=q_{l} r_{l-1}+0$ e o algoritmo termina $\operatorname{com} m d c(a, b)=r_{l-1}$.

\section{A.3 Teoremas úteis}

Teorema A.3.1. Se $m_{b} / 2^{n}$ for uma fração racional e b e r forem inteiros positivos que satisfazem

$$
\left|\frac{b}{r}-\frac{m_{b}}{2^{n}} \leq \frac{1}{2 r^{2}}\right|
$$

então b/r é uma convergência de uma fração continuada de $\frac{m_{b}}{2^{n}}$.

Teorema A.3.2. A função de Euler pode ser calculada para um inteiro positivo arbitrário $N$ da seguinte forma

$$
\varphi(N)=N \prod_{p}\left(\frac{p-1}{p}\right),
$$

onde p evolui sobre todos os fatores primos diferentes de $N$, incluindo o próprio $N$ se a é primo.

Duas consequência importantes é que $\varphi(N)=N-1$ se e somente se $N$ é primo. Se $N$ é uma composição de números, então $\varphi(N)<N-1$. 
Teorema A.3.3. Para um inteiro arbitrário $N>1$ se o $m d c(N, a)-1$ então segue que a congruência (ax) $\bmod N \equiv 1$ tem somente uma solução no sentido modulo $N$, caso contrário não há solução.

Teorema A.3.4. Pequeno Teorema de Fermat: Para um número primo arbitrário $N$ e $a \in \mathbb{Z}_{N}^{*}$

$$
\left(a^{N-1}\right) \quad \bmod N \equiv 1 .
$$

Teorema A.3.5. Teorema de Euler: Para um inteiro arbitrário $N>1$ e a $\in \mathbb{Z}_{N}^{*}$

$$
\left(a^{\varphi(N)}\right) \quad \bmod N \equiv 1
$$

Uma importante consequência deste teorema é que a função de Euler é multiplicativo, ou seja, $\varphi(a) \cdot \varphi(b)=\varphi(a b)$ sempre que $\operatorname{md} c(a, b)=1$.

Teorema A.3.6. Teorema Chinês do Resto: Assumindo o seguinte sistema de congruência para diferentes módulos

$$
\begin{gathered}
x \equiv a_{1}\left(\bmod m_{1}\right), \\
x \equiv a_{2}\left(\bmod m_{2}\right), \\
\vdots \\
x \equiv a_{l}\left(\bmod m_{l}\right) .
\end{gathered}
$$

Além disso, é suposto que $m_{i}$ e $m_{K}$ são co-primos tal que $m d c\left(m_{i}, m_{k}\right)=1$ se $i \neq k$. Então, existem a soluções $x_{0}$ para todas as congruências e nenhum par de congruência é congruente a qualquer outra no sentido módulo $m=\prod_{i=1}^{l} m_{i}$.

\section{A.4 Conceitos básicos de álgebra linear}

\section{A.4.1 Espaços vetoriais}

$V$ é chamado de um espaço vetorial $n$-dimensional sobre os números complexos se os seguintes critérios são satisfeitos: 
1.Elementos de $V$ são chamados vetores e compostos de uma sequência $n$ dimensional de números complexos $|v\rangle=\left[v_{1}, v_{2}, \ldots, v_{n}\right]^{T}, v_{i} \in \mathbb{C}$.

2.Existe uma operação chamada adição definida como $|a\rangle=|v\rangle+|b\rangle, a_{i}=v_{i}+b_{i}$. A adição não leva a um resultado fora do espaço vetorial;

3.Adição é associativa e comutativa e existe um vetor zero $\mathbf{0}$ para o qual $\forall|v\rangle \in V$, $|v\rangle+\mathbf{0}=|v\rangle$

4.Para cada elemento de $V$ há um aditivo inverso tal que $|v\rangle+(-|v\rangle)=\mathbf{0}$;

5.Existe uma operação chamada multiplicação escalar entre um número complexo $c$ e vetores tal que $|a\rangle=c \cdot|v\rangle, a_{i}=c \cdot v_{i}$.

\section{A.4.2 Bases e independência linear}

Os conceitos de bases e independência linear podem ser definidos da forma:

1. $\left|v_{1}\right\rangle, \ldots,\left|v_{m}\right\rangle$ são ditos vetores de expansão do espaço $m$-dimensional $V$, se $\forall|v\rangle \in$ $V,|v\rangle=\sum_{i} c_{i}\left|v_{i}\right\rangle, c_{i} \in \mathbb{C}$. Um espaço vetorial tem vários conjuntos de vetores de expansão;

2. $\left|v_{1}\right\rangle, \ldots,\left|v_{m}\right\rangle$ são ditos linearmente dependentes se $\left|c_{1}\right\rangle, \ldots,\left|c_{m}\right\rangle \in \mathbb{C}, c_{i} \neq 0$, tal que $\sum_{i} c_{i}\left|v_{i}\right\rangle=0$. Caso contrário, $\left\{\left|v_{i}\right\rangle\right\}$ é dito linearmente independente;

3.Se um conjunto de vetores de expansão de $V$ é linearmente independente, este é então chamado de uma base de $V$.

\section{A.4.3 Auto-vetores e autovalores}

$|u\rangle$ é dito um auto-vetor de um operador $U$ se:

$$
U|u\rangle=\omega_{u}|u\rangle
$$

onde $=\omega_{u} \in \mathbb{C}$ representa um autovalor de $U$.

Autovalores são obtidos pela chamada equação característica: 


$$
\operatorname{det}(\mathbf{U}-\omega \mathbf{I})-0 .
$$

Se um dado autovalor pertence a mais de um auto-vetor, então este é dito um autovalor degenerado.

\section{A.4.4 Operações básicas sobre vetores}

Transposição $\left({ }^{T}\right)$ de um vetor $|v\rangle$ produz um vetor-coluna e vice e versa;

Complexa conjugada $\left(^{*}\right)$ de um vetor $|v\rangle$ conjuga cada uma das coordenadas do vetor;

$\left.\operatorname{Adjunto}^{\dagger}\right)$ de um vetor $|v\rangle$ é definido da forma $|v\rangle=\left(|v\rangle^{T}\right)^{*}$;

Ortonormalidadeelementos de $\left\{\left|v_{i}\right\rangle\right\}$ são ditos ortnormais se tamanho igual a 1 e são mutalmente ortogonais, i.e., $\left\langle v_{j} \mid v_{j}\right\rangle=\delta(i-j)$;

Produto tensorial $(\otimes)$ de vetores é utilizado para unificar distintos espaços vetoriais. Se $\left\{\left|v_{i}\right\rangle \in V\right\}$ e $\left\{\left|w_{k}\right\rangle \in W\right\}$ são bases ortonormais então $\left\{\left|v_{i}\right\rangle \otimes\left|w_{k}\right\rangle\right\}$ forma uma base ortonormal para um espaço vetorial $V \otimes W$. Notações equivalentes para o produto tensorial são: $\left\{\left|v_{i}\right\rangle \otimes\left|w_{k}\right\rangle\right\},\left\{\left|v_{i}\right\rangle\left|w_{k}\right\rangle\right\}$ e $\left\{\left|v_{i} w_{k}\right\rangle\right\}$;

\section{A.5 Técnicas de cálculo numérico}

\section{A.5.1 Algoritmo de busca bissectiva}

O algoritmo de busca bissectiva é uma técnica de busca de dados frequentemente utilizada na solução de diversas tarefas computacionais. Esta é relativamente simples, apresenta fácil implementação e baixo custo computacional.

A busca bissectiva explora a existência de alguma ordem ou propriedade geral no espaço de busca. Considerando, como exemplo, a existência de uma lista numérica ordenada de forma crescente e de dimensão $N$, o algoritmo de busca bissectiva atua por meio das seguintes ações:

1.Escolha uma posição intermediária na sequência; 
2.Compare o valor buscado com aquele na posição escolhida;

3.Se os valores são idêntico, retorne o valor buscado e finalize;

4.Se o valor que está sendo buscado é menor que o valor comparado, vá a uma posição intermediária antes do ponto comparado e volte ao passo 2;

5.Se o valor que está sendo buscado é maior que o valor comparado, vá a uma posição intermediária após o ponto comparado e volte ao passo 2;

Sendo a lista ordenada, o algoritmo localiza o valor buscado com probabilidade igual a 1 e complexidade $O(\log N)$.

O conceito do algoritmo de busca bissectiva pode ser aplicado a outros casos semelhantes onde há alguma propriedade no espaço de busca. 


\section{APÊEDICE B - Fundamentos de mecânica quântica}

\section{B.1 Funções de onda e a equação de Schrödinger}

A física quântica estabelece uma interpretação ondulatória para a Natureza que pode ser resumida pela formulação:

iO conceito clássico de trajetória deve ser substituído pelo conceito de uma estado que varia no tempo. O estado quântico de uma partícula tal como o elétron é caracterizado por uma função de onda $\psi(\mathbf{r}, t)$, a qual contém toda informação possível de se obter sobre a partícula.

iiA função de onda $\psi(\mathbf{r}, t)$ é interpretada como uma amplitude de probabilidade da presença da partícula. Assim, as possíveis posições da partícula formam um continuum $\mathrm{d} \mathcal{P}(\mathbf{r}, t)$ da partícula estar no instante $t$ em um elemento de volume $d^{3} r=$ $\mathrm{d} x \mathrm{~d} y \mathrm{~d} z$ situado no ponto $\mathbf{r}$ deve ser proporcional a $d^{3} r$ portanto infinitesimal. $|\psi(\mathbf{r}, t)|^{2}$ é então a densidade de probabilidade correspondente, com

$$
\mathrm{d} \mathcal{P}(\mathbf{r}, t)=C|\psi(\mathrm{r}, t)|^{2} d^{3} r
$$

onde $C$ é uma constante de normalização.

iiiPara uma medida arbitrária de uma quantidade física

ao resultado deve pertencer ao conjunto de auto-valores $a$;

bcada auto-valor $a$ é associado a uma auto-estado, que é uma auto-função $\psi_{a}(\mathbf{r})$.

Esta é uma função tal que $\psi(\mathbf{r}, t)=\psi_{a}(\mathbf{r})$, sendo $t_{0}$ o tempo no qual a medida foi realizada; 
cpara qualquer $\psi(\mathbf{r}, t)$, a probabilidade $\mathcal{P}_{a}$ de encontrar $a$ para uma medida no tempo $t_{0}$ é encontrada por meio da decomposição de $\psi(\mathbf{r}, t)$ nos termos das funções $\psi_{a}(\mathbf{r})$, i.e.,

$$
\psi\left(\mathbf{r}, t_{0}\right)=\sum_{a} c_{a} \psi_{a}(\mathbf{r})
$$

Então,

$$
\mathcal{P}_{a}=\frac{\left|c_{a}\right|^{2}}{\sum_{a}\left|c_{a}\right|^{2}}
$$

dse uma medida produzir $a$, a função de onda imediatamente após a medida passará a ser $\psi^{\prime}\left(\mathbf{r}, t_{0}\right)=\psi_{a}(\mathbf{r})$.

ivequação que descreve a evolução temporal da função de onda $\psi(\mathbf{r}, t)$ pode ser escrita da forma abaixo e é conhecida como Equação de Schrödinger.

$$
\mathrm{i} \hbar \frac{\partial}{\partial t} \psi\left(\mathbf{r}, t_{0}\right)=-\frac{\hbar^{2}}{2 m} \Delta \psi(\mathbf{r}, t)+V(\mathbf{r}, t) \psi(\mathbf{r}, t)
$$

onde $m$ é a massa de uma partícula sujeita a um potencial $\mathbf{r}, t$ e $\Delta$ é o operador Laplaciano $\partial^{2} / \partial x^{2}+\partial^{2} / \partial y^{2}+\partial^{2} / \partial z^{2}$. O símbolo $\hbar$ refere-se a relação envolvendo a constante de Planck $h$ tal que $\hbar=h / 2 \pi=1,054589 \times 10^{-34}$ joule segundo.

\section{B.2 Notação de Dirac}

A cada função de onda do espaço das funções de onda se associa um vetor de estado de um espaço de estados. Utiliza-se para a representação do vetor o símbolo $|\cdots\rangle$ chamado ket por razões históricas. Dessa forma,

$$
\psi(\mathbf{r}) \in \mathfrak{J} \Longleftrightarrow|\psi\rangle \in \epsilon_{r} .
$$

\section{B.3 Postulados fundamentais da mecânica quântica}

Postulado 1. Espaço de estados: o estado real de um sistema físico fechado qualquer pode ser descrito por meio do chamado vetor de estado $\mathrm{v}$ tendo coeficientes complexos 
e tamanho unitário no espaço de Hilbert $\mathrm{V}$, i.e., um espaço vetorial complexo linear (espaço de estados) possuindo um produto interno.

Postulado 2. Evolução: a evolução de qualquer sistema físico fechado no tempo pode ser caracterizada por meio de transformações unitárias dependendo somente do tempo de início e fim da evolução.

Postulado 3. Medida: qualquer medida quântica pode ser descrita por meio de uma operador de medida $M_{m}$, onde $m$ refere-se ao possível resultado da medida. A probabilidade de se encontrar m por meio de uma medida estando o sistema no estado v pode ser calculado por

$$
P\langle m \mid v\rangle=\mathrm{v}^{\dagger} M_{m}^{\dagger} M_{m} \mathrm{v}
$$

com o sistema depois da medida indo para o estado

$$
\mathrm{v}^{\prime}=\frac{M_{m} \mathrm{v}}{\sqrt{\mathrm{v}^{\dagger} M_{m}^{\dagger} M_{m} \mathrm{v}}} .
$$

Devido a teoria clássica de probabilidades, é necessário que

$$
\sum_{m} P\langle m \mid v\rangle \equiv \sum \mathrm{v}^{\dagger} M_{m}^{\dagger} M_{m} \mathrm{v}=1,
$$

sendo assim os operadores de medida devem satisfazer a relação de completeza expressa por

$$
\sum_{m} M_{m}^{\dagger} M_{m} \equiv \mathrm{I}
$$

onde I é a matriz identidade.

Postulado 4. Composição de sistemas: o espaço de estado de uma composição de sistemas físicos $W$ pode ser determinado usando o produto tensorial dor sistemas individuais $W=V \otimes Y$. Além disso, uma vez definido $\mathrm{v} \in V$ e $\mathrm{y} \in Y$ então o estado conjunto da composição do sistema é $\mathrm{w}=\mathrm{v} \otimes \mathrm{y}$. 


\section{B.4 Descrição partícula-onda para a luz}

Os fótons polarizados podem ser obtidos a partir de feixes de luz e filtro polarizadores ou cristais polarizadores. A tecnologia atual consegue produzir LEDs e laser capazes de emitir um único fóton, possibilitando a emissão de um fóton polarizado de acordo com uma direção específica do polarizador. O princípio da incerteza indica que para um fóton polarizado em uma direção $\alpha$ que passa por meio de um polarizador orientado na direção $\beta$ se comportará probabilisticamente com probabilidade $\cos ^{2}(\alpha-\beta)$ de ser absorvido ou transmitido. O caso determinístico de absorção completa ou transmissão completa somente ocorre quando $\beta$ for perpendicular ou paralelo à direção $\alpha$ respectivamente. Após se realizar a medida com o polarizador na direção $\beta$, o fóton resultante emerge com polarização exatamente $\beta$, não sendo possível utilizando um terceiro polarizador em uma terceira direção obter mais informação sobre o fóton originário. Uma possível forma de se driblar tal limitação seria gerar uma cópia do fóton e então executar a medida sobre este. Contudo, devido às leis da mecânica quântica não é possível clonar um fóton ou qualquer estado físico sem alterá-lo já que o processo de cópia inerentemente representa um processo de medida. Montando um aparato que permita produzir fótons polarizados em duas bases distintas, polarização horizontal-vertical e polarização diagonal, respectivamente 0 e 90 graus e 45 e 135 graus, têm se então, de acordo com a estrutura matemática da mecânica quântica um sistema em que um fóton polarizado em uma base quando medido em outra base não revelará informação alguma sobre seu estado original.

\section{B.4.1 Distribuição de Poisson}

Na teoria da probabilidade e na estatística, a distribuição de Poisson é uma distribuição de probabilidade discreta. Ela expressa, por exemplo, a probabilidade de um certo número de eventos ocorrerem num dado período tempo, caso estes ocorram com uma taxa média conhecida e caso cada evento seja independente do tempo decorrido desde o último evento. A distribuição foi descoberta por Siméon-Denis Poisson (1781 - 1840) e publicada, conjuntamente com a sua teoria da probabilidade, em 1838 no seu trabalho Recherches sur la probabilité des jugements en matières criminelles et matière civile (Inquérito sobre a probabilidade em julgamentos sobre matérias criminais e civis). $\mathrm{O}$ 
trabalho focava-se em certas variáveis aleatórias $\mathrm{N}$ que contavam, entre outras coisas, o número de ocorrências discretas (por vezes chamadas de "chegadas") que tinham lugar durante um intervalo de tempo de determinado comprimento. A probabilidade de que existam exactamente $\mathrm{k}$ ocorrências ( $\mathrm{k}$ sendo um inteiro não negativo, $k=0,1,2, \ldots$ ) é

$$
f(k ; \lambda)=\frac{e^{-\lambda} \lambda^{k}}{k !}
$$

e além disso,

$$
P[X(t)=K]=\frac{e^{-\lambda t} \lambda t^{k}}{k !}
$$

onde $e$ é base do logaritmo natural $(e=2.71828 \ldots), k$ ! é o fatorial de $k, \lambda$ é um número real, igual ao número esperado de ocorrências que ocorrem num dado intervalo de tempo. Por exemplo, se o evento ocorre a uma média de 4 minutos, e estamos interessados no número de eventos que ocorrem num intervalo de 10 minutos, usariámos como modelo a distribuição de Poisson $\operatorname{com} \lambda=10 / 4=2.5$. Como função de $k$, esta é a função massa de probabilidade. A distribuição de Poisson pode ser derivada como um caso limite da distribuição binomial.

\section{B.5 Princípio da incerteza de Heisenberg}

O princípio da incerteza de Heisenberg consiste num enunciado da mecânica quântica, formulado inicialmente em 1927 por Werner Heisenberg, impondo restrições à precisão com que se podem efetuar medidas simultâneas de uma classe de pares de observáveis. Pode-se descrever o princípio da incerteza nos seguintes termos: o produto da incerteza associada ao valor de uma coordenada $x_{i}$ e a incerteza associada ao seu correspondente momento linear $p_{i}$ não pode ser inferior, em grandeza, à constante de Planck normalizada, ou seja,

$$
\Delta x_{i} \Delta p_{i} \geq \frac{h}{4 \pi}
$$

onde $h$ é a Constante de Planck.

A explicação disso é fácil de se entender, e mesmo intuitiva, embora o raciocínio 
clássico e os aspectos formais da análise matemática tenham levado os cientistas a pensarem diferentemente por muito tempo. Quando se quer encontrar a posição de um elétron, por exemplo, é necessário fazê-lo interagir com algum instrumento de medida, direta ou indiretamente. Por exemplo, faz-se incidir sobre ele algum tipo de radiação. Tanto faz aqui que se considere a radiação do modo clássico - constituída por ondas eletromagnéticas - ou do modo quântico - constituída por fótons. Se se quer determinar a posição do elétron, é necessário que a radiação tenha comprimento de onda da ordem da incerteza com que se quer determinar a posição. Neste caso, quanto menor for o comprimento de onda (maior frequência) maior é a precisão. Contudo, maior será a energia cedida pela radiação (onda ou fóton) em virtude da relação de Planck entre energia e frequência da radiação

$$
E=h \cdot v,
$$

e o elétron sofrerá um recuo tanto maior quanto maior for essa energia, em virtude do efeito Compton. Como consequência, a velocidade sofrerá uma alteração não de todo previsível, ao contrário do que afirmaria a mecânica clássica. Argumentos análogos poderiam ser usados para se demonstrar que ao se medir a velocidade com precisão, alterar-se-ia a posição de modo não totalmente previsível. Resumidamente, pode-se dizer que tudo se passa de forma que quanto mais precisamente se medir uma grandeza, forçosamente mais será imprecisa a medida da grandeza correspondente, chamada de canonicamente conjugada

\section{B.6 Teorema da não-clonagem}

Nesta seção são apresentados o teorema da não-clonagem e sua demonstração. O teorema estabelece que é impossível copiar um estado quântico desconhecido, i.e., é impossível copiar um estado quântico sem alterar seu estado inicial. Isso pode ser visto da seguinte forma: suponha que se tenha uma máquina quântica com dois compartimentos $A$ e $B$. O compartimento de dados, $A$, inicia em um estado quântico puro, porém desconhecido $|\psi\rangle$. Esse é o estado que se quer copiar para o comparimento-alvo, B. Supõe-se que o compartimento-alvo inicia em um estado puro $|s\rangle$. Portanto, o estado inicial da máquina de clonagem é: 


$$
|\psi\rangle \otimes|s\rangle
$$

Alguma evolução unitária implementa idealmente o procedimento de cópia:

$$
|\psi\rangle \otimes|s\rangle \longrightarrow U(|\psi\rangle \otimes|s\rangle)=|\psi\rangle \otimes|s\rangle
$$

Suponha que sejam feitas cópias de dois estados, $|\psi\rangle$ e $|\varphi\rangle$. Portanto, têm-se:

$$
\begin{gathered}
U(|\psi\rangle \otimes|s\rangle)=|\psi\rangle \otimes|s\rangle \\
U(|\varphi\rangle \otimes|s\rangle)=|\varphi\rangle \otimes|s\rangle .
\end{gathered}
$$

Tomando o produto interno das duas equações, têm-se:

$$
\langle\psi \mid \varphi\rangle=(\langle\psi \mid \varphi\rangle)^{2}
$$

Mas $x=x^{2}$ só tem duas soluções, $x=0$ e $x=1$, e portanto ou $|\psi\rangle=|\varphi\rangle$ ou $|\psi\rangle$ e $|\varphi\rangle$ são ortogonais. Logo a máquina de clonagem só pode copiar estados ortogonais entre si, e portanto uma máquina de clonagem geral é impossível. A máquina não pode, por exemplo, clonar os estados $|\psi\rangle=|0\rangle \mathrm{e}|\varphi\rangle=(|0\rangle+|1\rangle / \sqrt{2})$, pois eles são não-ortogonais.

O teorema da não-clonagem estabelece então de forma geral que mesmo máquinas de clonagem não-unitárias não podem copiar estados puros não-ortogonais, a menos que se tolere uma perda finita da fidelidade dos estados copiados. 


\section{APÊNDICE C - Códigos de implementação}

Neste apêndice são mostrados excertos dos códigos computacionais utilizados no desenvolvimento do ambiente de simulação para o protocolo BB84 e dos procedimentos de reconciliação de informação, considerando o algoritmo Cascade modificado, e de amplificação de privacidade discutidos ao longo deste trabalho.

Os códigos se referem a linguagem de programação $\mathrm{C}++$.

\section{C.1 Escopo das funções de implementação}

Como principal objetivo da definição das funções propostas para o módulo, está a simulação de forma realista do protocolo de criptografia quântica baseado no protocolo BB84 e dos desenvolvimentos teóricos realizados anteriormente. Para isso ocorre a simulação das ações de Alice, Bob e Eva e dos canais entre eles. A seguir é mostrado como foram definidas estas funções de acordo com esse objetivo.

O módulo utiliza as classes e funções com os seguintes protótipos para implementar o funcionamento do protocolo quântico:

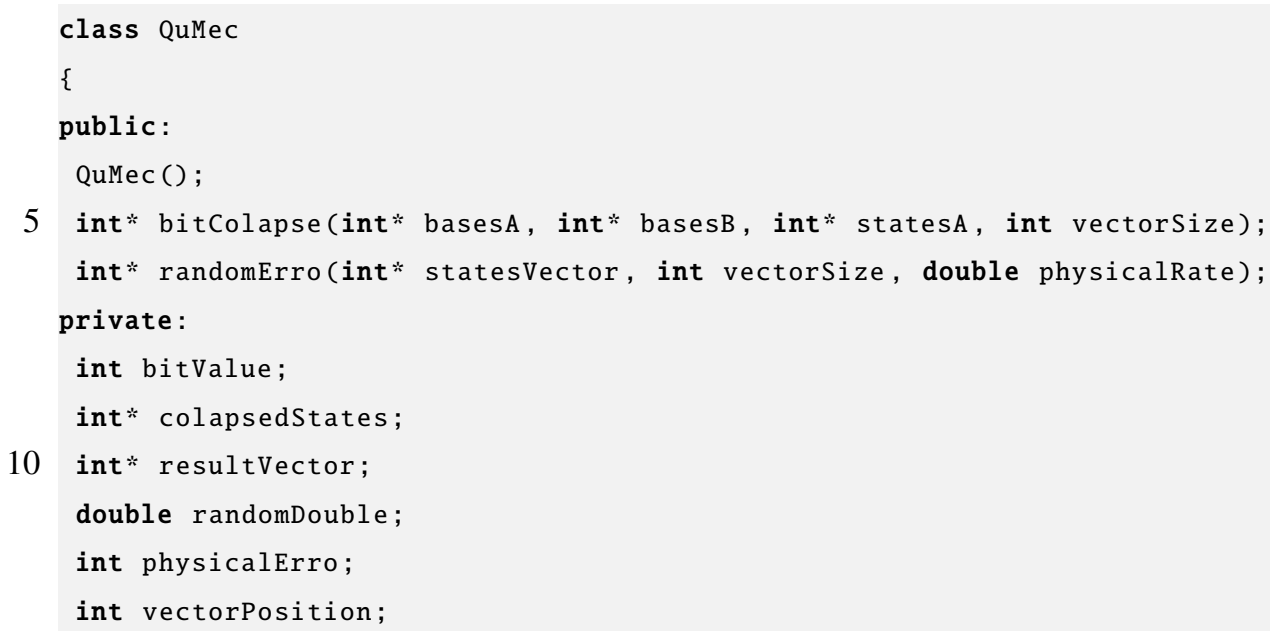


\};

15

class GetRandom

\{

public:

GetRandom();

20 int* randomSequence(int vectorSize);

private:

double randomDouble;

int* resultVector;

int bitValue;

$25\}$;

class VerificationTools

\{

public:

30 VerificationTools();

double checkErro (int* vectorA, int* vectorB, int vectorSize);

int* basesVerification (int* basesA, int* basesB, int vectorSize);

int* randomPosition(int* vectorA, int vectorSize, int randomSize);

double erroBases(int* vectorA, int* vectorB, int* vectorC, int vectorSize);

35 void printKey (int* vectorStates, int vectorSize);

int* bitsDivulged(int* basesCorrect, int vectorSize, int testSize);

private:

double errocount, erroTested, erroRate;

int* correctBases;

40 int vectorPosition;

int* resultVector;

\} ;

class Reconciliation

45\{

public:

Reconciliation();

int parityCheck (int* vectorA, int* vectorB, int vectorPosition, int vectorSize);

int parityValue(int* vector, int vectorPosition, int vectorSize);

50 void binaryAction(int* vectorA, int* vectorB, int vectorPosition,

int blockSize, int* parityBits);

void biconfirmAction(int* vectorA, int* vectorB, int randomBlock,

int vectorSize, int blockSize, int* parityBits);

int* universalHash(int* vectorA, int vectorSize, int hashSize, int randomBlock);

55 private:

int parityErro;

\}; 